\title{
Commissioning and first data taking experience with the Belle II pixel vertex detector
}

\author{
Dissertation \\ zur Erlangung des mathematisch-naturwissenschaftlichen Doktorgrades \\ „Doctor rerum naturalium“ \\ der Georg-August-Universität Göttingen \\ im Promotionsprogramm ProPhys \\ der Georg-August University School of Science (GAUSS)
}

vorgelegt von

Harrison Schreeck

aus Hildesheim

Göttingen, 2020 
Betreuungsausschuss

Prof. Dr. Ariane Frey

Prof. Dr. Stan Lai

Mitglieder der Prüfungskommission:

Referent: $\quad$ Prof. Dr. Ariane Frey

II. Physikalisches Institut, Georg-August-Universität Göttingen

Koreferent: PD Dr. Jörn Große-Knetter

II. Physikalisches Institut, Georg-August-Universität Göttingen

Weitere Mitglieder der Prüfungskommission:

Prof. Dr. Stan Lai

II. Physikalisches Institut, Georg-August-Universität Göttingen

Prof. Dr. Wolfram Kollatschny

Institut für Astrophysik, Georg-August-Universität Göttingen

Prof. Dr. Hans-Christian Hofsäss

II. Physikalisches Institut, Georg-August-Universität Göttingen

Dr. Carsten Niebuhr

Deutsches Elektronen-Synchrotron DESY, Hamburg

Tag der mündlichen Prüfung: 22.05.2020

Referenz: II.Physik-UniGö-Diss-2020/03 


\title{
Commissioning and first data taking experience with the Belle II pixel vertex detector
}

\begin{abstract}
The Belle II experiment at the SuperKEKB particle accelerator at KEK in Tsukuba (Japan) is the successor to the Belle experiment, which played a crucial role in the discovery of $\mathrm{CP}$ violation in the B-Meson system. The vertex detector of Belle II was upgraded with two layers of a DEPFET based pixel detector (PXD) to improve the vertex resolution compared to Belle. This thesis gives an overview of the PXD and its individual components. The focus of the work lies on the transition from a pure laboratory system to a fully integrated one in Belle II. Before the final detector components were installed at Belle II, a pre-experiment called phase 2 was conducted to test the new parts. A description of the necessary steps and changes to the hardware configuration and software as well as a report on the operation experiences gathered during phase 2 is given in this thesis.

Additionally, this thesis reports on the effects of ionising radiation on the PXD sensors and their DEPFET matrices. This includes a description of the radiation sources at particle accelerators in general and SuperKEKB in particular. In order to get a better understanding of the radiation effects seen on the PXD sensors during the phase 2 operation an X-ray irradiation campaign was performed. In this campaign a single PXD sensor belonging to the same batch as the final sensors was irradiated. The DEPFETs of the PXD sensor received a dose of $266 \mathrm{kGy}$ during this campaign. A detailed description of the campaign and the results gathered from it are presented.
\end{abstract}





\section{Commissioning and first data taking experience with the Belle II pixel vertex detector}

\section{Zusammenfassung}

Das Belle II Experiment am SuperKEKB Teilchenbeschleuniger am KEK in Tsukuba (Japan) ist der Nachfolger des Belle Experiments, welches eine entscheidende Rolle bei der Entdeckung der CP Verletzung im B-Mesonen System gespielt hat. Der Vertexdetektor von Belle II wurde mit einem zweilagigen Pixeldetektor (PXD), der auf DEPFET Technologie basiert, verbessert um die Vertexauflösung gegenüber Belle zu erhöhen. Diese Arbeit gibt einen Überblick über den PXD und seine einzelnen Komponenten. Der Fokus der Arbeit liegt dabei auf dem Übergang von einem Labor basierten System zu einem komplett integrierten bei Belle II. Bevor die finalen Detektorkomponenten bei Belle II installiert wurden, wurde ein Vorexperiment (Phase 2) durchgeführt um die neuen Komponenten zu testen. Diese Arbeit gibt eine Beschreibung der notwendigen Schritte und Änderungen an Hard- und Software sowie einen Bericht zu den Erfahrungen, die während der Phase 2 Operation gesammelt wurden.

Zusätzlich enthält diese Arbeit einen Abschnitt über die Effekte von ionisierender Strahlung auf PXD Sensoren und ihre DEPFET Matrizen. Dabei wird auch auf Strahlungsquellen bei Teilchenbeschleunigern im Allgemeinen und SuperKEKB im Besonderen eingegangen. Um ein besseres Verständnis für die auf den PXD Sensoren während Phase 2 beobachteten Strahlungseffekte zu gewinnen, wurde eine Bestrahlungskampagne mit einer Röntgenröhre durchgeführt. Bei dieser Kampagne wurde ein einzelner PXD Sensor, der aus der gleichen Produktionscharge stammt wie die Sensoren, die für Belle II genutzt werden, bestrahlt. Die DEPFETs des PXD Sensors haben dabei eine Dosis von 266 kGy angesammelt. Eine detaillierte Beschreibung der Messkampagne sowie der gewonnenen Resultate erfolgt in dieser Arbeit. 

$\begin{array}{ll}\text { 1. Motivation and Overview } & 1\end{array}$

2. Introduction 3

2.1. Standard Model of Particle Physics . . . . . . . . . . . . . . . . . . 3

2.1.1. Quantum Electrodynamics (QED) . . . . . . . . . . . . 4

2.1.2. Quantum Flavourdynamics (QFD) . . . . . . . . . . . . . 5

2.1.3. Quantum Chromodynamics (QCD) . . . . . . . . . . . . 6

2.1.4. Higgs boson . . . . . . . . . . . . . . . . . . . . 6

2.2. CKM Matrix ....................... . . 7

2.3. Symmetries . . . . . . . . . . . . . . . . . . . 10

2.4. CP Violation . . . . . . . . . . . . . . . . . . . 10

2.5. B-Physics and B-Factories . . . . . . . . . . . . 13

$\begin{array}{lr}\text { 3. SuperKEKB and Belle2 } & 17\end{array}$

3.1. SuperKEKB . . . . . . . . . . . . . . . . . . . . . 17

3.1.1. Backgrounds . . . . . . . . . . . . . . . . . . 19

3.2. The Belle II experiment . . . . . . . . . . . . . . . . . . . . 24

3.2.1. Vertex Detector $(\mathrm{VXD}) \ldots \ldots \ldots \ldots \ldots$

3.2.2. Central Drift Chamber $(\mathrm{CDC}) \quad \ldots \ldots \ldots \ldots . \ldots . \ldots 25$

3.2.3. Particle Identification . . . . . . . . . . . . . . . . 26

3.2.4. Electromagnetic Calorimeter (ECL) . . . . . . . . . . . . 27

3.2.5. $K_{L}$ and muon detector $(\mathrm{KLM}) \ldots \ldots \ldots . \ldots 28$

4. Pixel Vertex Detector for Belle II 29

4.1. Semiconductors . . . . . . . . . . . . . . . . . . 29

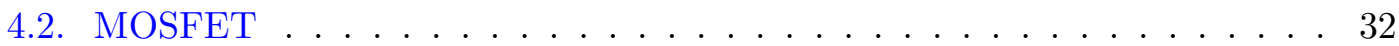

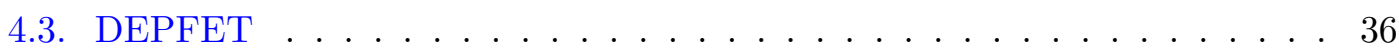

4.4. Radiation Damage Effects on the DEPFETs . . . . . . . . . . . . 38

4.4.1. Bulk Damage . . . . . . . . . . . . . . . . . . . . . 38 
4.4.2. Oxide Damage . . . . . . . . . . . . . . . . . . . . . . 39

4.4.3. Oxide Damage Annealing . . . . . . . . . . . . . . . . 41

4.5. PXD half-ladder and Front-end readout . . . . . . . . . . . . . . . . 42

4.5.1. DEPFET Matrix . . . . . . . . . . . . . . . . 43

4.5.2. Switcher . . . . . . . . . . . . . . 46

4.5.3. Drain Current Digitizer (DCD) . . . . . . . . . . . . . 46

4.5.4. Data Handling Processor (DHP) . . . . . . . . . . . . . . 49

4.6. Back-end readout and Data Acquisition (DAQ) . . . . . . . . . . . . 52

4.6.1. DHH Components . . . . . . . . . . . . . . . 52

4.6.2. Full DHH System and Global DAQ Integration . . . . . . . . . . 53

4.6.3. Local PXD DAQ and Laboratory Readout . . . . . . . . . . . . . 55

4.7. Power Supply and Sevices . . . . . . . . . . . . . . . . . . . . . . . . 55

4.8. Hybrid5 Sensors . . . . . . . . . . . . . . . 57

5. Slow Control and Calibration Software 59

5.1. EPICS . . . . . . . . . . . . . . . . . . . 59

5.2. ASIC Configuration . . . . . . . . . . . . . . . . 62

5.3. Configuration Database . . . . . . . . . . . . . . . . 62

5.4. PXD Startup Sequence . . . . . . . . . . . . . . . . . . . 63

5.4.1. Pedestal and 2-Bit DAC Offset Correction Upload . . . . . . . . 65

5.5. Calibration Software Framework . . . . . . . . . . . . . . . 68

5.6. Utility IOC . . . . . . . . . . . . . . . . . . . . . . . . . 69

5.7. Calibration IOC . . . . . . . . . . . . . . . 70

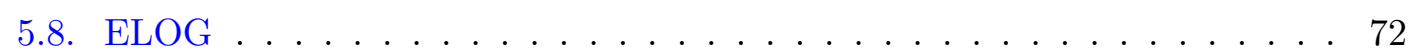

5.8.1. ELOG Usage in the Laboratory . . . . . . . . . . . . . . . . . . 73

5.8.2. Automation of Belle II Run Logbook Entries . . . . . . . . . . . . 76

$\begin{array}{ll}\text { 6. Phase } 2 \text { Commissioning } & \mathbf{7 9}\end{array}$

6.1. BEAST II . . . . . . . . . . . . . . . . . . . . . . . 79

6.2. Pixel Vertex Detector Preparations in the Laboratory . . . . . . . . . . . 82

6.3. Commissioning and Operation of the Pixel Vertex Detector . . . . . . . . 86

6.4. Data Taking Experience and Analysis . . . . . . . . . . . . . . . . 89

6.4.1. Cluster Charge Analysis . . . . . . . . . . . . . . . . . 89

6.4.2. Sensor Noise . . . . . . . . . . . . . . . . . . 93

6.4.3. Signal-to-Noise Performance . . . . . . . . . . . . . . . . . 93

6.5. Total Ionising Dose Estimation . . . . . . . . . . . . . . . . . 98

$\begin{array}{ll}\text { 7. Irradiation Experiment } & 109\end{array}$

7.1. Irradiation Setup . . . . . . . . . . . . . . . . . . . . 109

7.1.1. Mechanics and Cooling . . . . . . . . . . . . . . 110

7.2. X-ray Spectrum and Geant4 Simulation . . . . . . . . . . . . . . 113

7.3. Dosimetry . . . . . . . . . . . . . . . . . . . . 116

7.3.1. Direct Measurement of the X-ray Spectrum . . . . . . . . . . . . 119

7.4. Irradiation and Measurement Procedure . . . . . . . . . . . . . . . 120 
7.5. Threshold Shifts . . . . . . . . . . . . . . . . . . . 122

7.6. Threshold Shift Comparison . . . . . . . . . . . . . . . 127

7.7. ASIC Performance . . . . . . . . . . . . . . . . . . 128

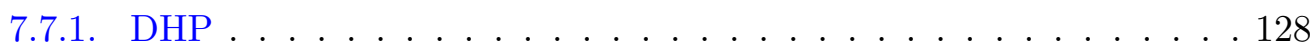

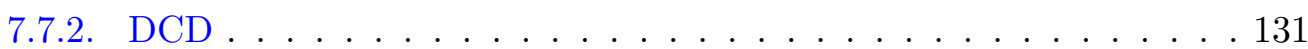

7.8. Radioactive Source Measurements . . . . . . . . . . . . . . . 133

7.8.1. Energy Calibration and DEPFET Working Point Evolution . . . . 135

7.8.2. Signal-to-Noise Ratio . . . . . . . . . . . . . . . . . . . . . . . 139

7.9. Efficiency Measurements with Beam Test Data . . . . . . . . . . . . . 140

$\begin{array}{ll}\text { 8. Conclusion } & 145\end{array}$

$\begin{array}{ll}\text { Bibliography } & 149\end{array}$

$\begin{array}{ll}\text { Appendices } & 159\end{array}$

$\begin{array}{ll}\text { A. ADC Transfer Curves } & 161\end{array}$

A.1. Transfer Curve Examples . . . . . . . . . . . . . . . . . . . 162

B. Total lonizing Dose Estimation 165

B.1. Residuals . . . . . . . . . . . . . . . . . . 165

$\begin{array}{lr}\text { C. Irradiation Campaign } & 169\end{array}$

C.1. System Gain Evolution . . . . . . . . . . . . . . . . . . . . . . 169

C.2. Signal-to-Noise Evolution . . . . . . . . . . . . . . . . 171 

CHAPTER 1

Motivation and Overview

The goal of physics has always been the description and understanding of the world around us. In order to do that theoretical models are developed and tested in practical measurements. Particle physics is no exception to this general approach. It aims at understanding the fundamental forces of nature by studying elementary particles and how they interact. While the standard model of particle physics gives exceptionally good descriptions of most of the observed phenomena, there are still various open questions that cannot be answered by our current models in a satisfactory manner.

One of the most interesting questions is the large asymmetry of matter and anti-matter in the universe. The standard model answers this question partly but an explanation of the vast excess of matter in the known universe is still out of our grasp. Other topics that keep physicists occupied are dark matter and of course the inclusion of gravity in the quantum field theories that describe all other forces of nature so successfully.

The experiments needed to study these subatomic particles have grown significantly in size and complexity over the last decades. When the first particles like the electron and later the positron where discovered, the experiments were conducted by individuals or small groups of physicists using small-scale experiments like bubble chambers.

Nowadays huge particle accelerators are built underground to collide particles and record the results of these collisions with enormous detectors. These accelerators and the particle detectors are designed, constructed, maintained and operated by hundreds of people organised in large international collaborations. Through research and development the accelerators are able to reach higher energies and collision rates requiring faster and more precise detectors to record the collision events.

The innermost part of nearly all detectors is usually a tracking device used to reconstruct the tracks of the particles created in these collisions and their decay vertices. The Belle II detector is particularly interesting in this aspect as it uses a pixel detector based on DEPFET technology which is used here for the very first time in a particle 


\section{Motivation and Overview}

detector. The DEPFET collaboration has been working for over 10 years on this pixel detector. The first sensors were finally installed in the Belle II detector in 2017 for a pre-experiment called phase 2 .

This thesis describes the DEPFET pixel detector (PXD) system with all its components from a hardware and software perspective. The transition from a laboratory system to a fully integrated system at Belle II will be shown and a report on the operational experience will be given.

In the first chapters the aforementioned standard model of particle physics is introduced and its key features are discussed. A particular focus lies on the phenomena of $\mathrm{CP}$ violation which explains to some degree the matter anti-matter asymmetry in the universe and the physics processes that are studied at Belle II. This includes a small outlook on possible extensions of the standard model.

After that a detailed description of the SuperKEKB accelerator and the Belle II detector is given. The peculiarities of the SuperKEKB are highlighted in this chapter especially compared to its predecessor KEKB. The description of Belle II introduces all sub-systems of the detector including the vertex detector.

The third chapter focuses on the DEPFET pixel detector itself. At first a general introduction to semiconductor detectors is given before field effect transistors are described which leads to an explanation of the DEPFET technology and how it is used for the pixel detector at Belle II. Furthermore the chapter reports on the front-end read out electronics as well as the services required to operate a DEPFET pixel sensor.

The following chapter is dedicated to the software side of the pixel detector. The various software frameworks required for the operation and calibration of the sensors are introduced and explained. This includes a description of the EPICS system, which builds the foundation of the entire software framework. Additionally, a report on software components that were developed as a part of this thesis work is given.

Chapter six covers the so-called phase 2 commissioning, the first operation of final DEPFET sensors at Belle II. The first half of the chapter discusses the challenges of the commissioning process and which adaptations from software and hardware side were required to operate the sensors outside of a controlled laboratory environment. The second half focuses on the performance of the sensors during the operation. This part also contains an estimation of the ionising dose received by the PXD.

Chapter seven reports on an X-ray irradiation campaign during which the DEPFETs of a single PXD half-ladder sensor were irradiated to a dose of $266 \mathrm{kGy}$. The results of the various measurements are presented in detail and compared with previous irradiation campaigns including the results from the phase 2 operation presented in the previous chapter. At the end of the chapter results from two beam test campaigns are discussed. The beam tests were done before and after the irradiation to investigate whether the hit detection efficiency suffered due to the irradiation.

The last chapter serves as the conclusion of this thesis and sums up all results. Furthermore, an outlook on the phase 3 operation is given. 
CHAPTER 2

Introduction

In this chapter an introduction to the field of particle physics will be given starting with a summary of the standard model of particle physics. Without going into too much details about the underlying mathematical formalisms the key points of the standard model will be presented together with the historically most important experiments and observations. As this thesis focuses on the pixel detector for the Belle II experiment a special focus lies on so-called b-quark physics. Therefore the concept of "B-Factories" will be introduced and explained.

\subsection{Standard Model of Particle Physics}

For over 40 years the standard model of particle physics has successfully described the interactions of elementary particles and is one of the best tested theories to date. It describes three of the four forces of nature, the electromagnetic force, the weak force and the strong force. The inclusion of gravity remains a challenge but as elementary particles have very low masses, it can be neglected for practically all measurements. Descriptions of the standard model can be found in various textbooks, the following overview is mainly based on [1].

The theoretical background for the standard model is Quantum Field Theory (QFT), which is a relativistic description of quantum mechanics and can therefore be used to describe microscopic particles at high velocities/energies. A fundamental property of elementary particles is their spin, which is also used to classify them into two groups, fermions and bosons. The former have a half-integer spin value while the latter have a full-integer value. A list of all fermions in the standard model can be found in table 2.1. In total there are 12 fermions, six leptons and six quarks. While they all have the same spin, they differ in mass, electric charge and the forces they interact with. Quarks are 


\section{Introduction}

the only particles that interact with the strong force. The fermions are further classified in three generations. Across these generations one particle "type" differs only in its mass, the muon for example has the same quantum numbers as the electron but its mass is over 200 times larger. For all fermions there is an anti-particle which has the same properties except for their charge related quantum numbers. The anti-particle for the electron is the positron which has an electric charge of +1 instead of -1 .

All other particles are composites of two or three quarks, called mesons and baryons. The lightest meson is the pion $\pi^{0}$, which consists of an up and anti-up quark (or down and anti-down). The proton is an example for a baryon with the quark content uud. There are, of course, many more mesons and baryons, forming what is sometimes called a particle zoo.

\begin{tabular}{rllllr}
\hline \multirow{2}{*}{ Fermion } & Name & Symbol & Generation & EM charge & Mass $\left[\mathrm{MeV} / \mathrm{c}^{2}\right]$ \\
\hline \multirow{6}{*}{ Lepton } & electron & $e^{-}$ & I & -1 & $0.5109989461(31)$ \\
& muon & $\mu^{-}$ & II & -1 & $105.6583745(24)$ \\
& tau & $\tau^{-}$ & III & -1 & $1776.86(12)$ \\
& electron neutrino & $\nu_{e}$ & I & 0 & $\approx 0$ \\
& muon neutrino & $\nu_{\mu}$ & II & 0 & $\approx 0$ \\
& tau neutrino & $\nu_{\tau}$ & III & 0 & $\approx 0$ \\
\hline \multirow{4}{*}{ Quark } & up & $u$ & I & $+2 / 3$ & $2.16_{-0.26}^{+0.49}$ \\
& charm & $c$ & II & $+2 / 3$ & $1270(20)$ \\
& top & $t$ & III & $+2 / 3$ & $172900(400)$ \\
& down & $d$ & I & $-1 / 3$ & $4.67_{-0.17}^{+0.48}$ \\
& strange & $s$ & II & $-1 / 3$ & $93_{-5}^{+11}$ \\
& bottom & $b$ & III & $-1 / 3$ & $4180_{-20}^{+30}$ \\
\hline
\end{tabular}

Table 2.1.: Fermions in the standard model and their properties. Not shown are the antiparticles which have the opposite electric charge but are identical otherwise. Values for the masses taken from [2].

\subsubsection{Quantum Electrodynamics (QED)}

As mentioned before the standard model covers three forces of nature. These forces are mediated by gauge bosons. A list of all gauge bosons can be found in table 2.2. The gauge boson of the electromagnetic force is the massless photon, it interacts with all particles that carry an electric charge. That means that neutrinos do not feel the electromagnetic force while all other fermions do. Electromagnetic interactions are described in the theory of Quantum Electrodynamics (QED). In 1960 Richard Feynman introduced a way to graphically represent these interactions using what is called Feynman diagrams. 
An example of such a diagram can be seen in fig. 2.1. The same formalism can be used to describe the other forces in the standard model as well. These diagrams can easily be translated into mathematical expressions to be evaluated.

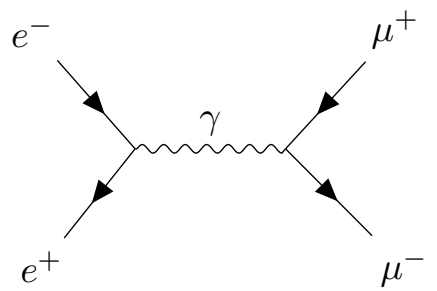

Figure 2.1.: Simple QED Feynman diagram showing the interaction of an incoming electron and positron through a photon, producing a muon/anti-muon pair.

\subsubsection{Quantum Flavourdynamics (QFD)}

Quantum Flavourdynamics (QFD) describes the weak force which has two gauge bosons, the $\mathrm{W}^{ \pm}$boson and the $\mathrm{Z}$ boson. The former comes in a positively and negatively charged form. In contrast to the photon they are not massless but have a mass of $\approx 80 \mathrm{GeV}\left(\mathrm{W}^{ \pm}\right)$and $\approx 90 \mathrm{GeV}(\mathrm{Z})$. Another key difference is that they interact with all fermions, including the neutrinos. As the $\mathrm{W}$ is electrically charged and the $\mathrm{Z}$ is not, weak interactions are divided into charged and neutral current interactions. While neutral current interactions do not allow flavour changes, charged current interactions do. Another important distinction between them is the fact that the $\mathrm{W}$ boson only interacts with left-handed particles and right-handed anti-particles. The corresponding property of a particle is called chirality. For massless particles the chirality is equivalent to the helicity of a particle, which describes whether the spin of the particle is oriented in the same or opposite direction of its movement. For massive particles, however, the helicity depends on the observer, as there is always an inertial frame of reference in which the particle moves "backwards". Mathematically, this behaviour is described by the introduction of left-handed doublets to which the $\mathrm{W}$ boson couples:

$$
\left(\begin{array}{l}
u \\
d
\end{array}\right)_{L},\left(\begin{array}{l}
c \\
s
\end{array}\right)_{L},\left(\begin{array}{l}
t \\
b
\end{array}\right)_{L},\left(\begin{array}{c}
\nu_{e} \\
e
\end{array}\right)_{L},\left(\begin{array}{c}
\nu_{\mu} \\
\mu
\end{array}\right)_{L},\left(\begin{array}{c}
\nu_{\tau} \\
\tau
\end{array}\right)_{L}
$$

Figure 2.2 shows Feynman diagrams of charged and neutral current interactions. Here the aforementioned flavour change during the $\mathrm{W}^{-}$interaction is visible $\left(\mu^{-} \rightarrow \nu_{\mu}\right)$. 

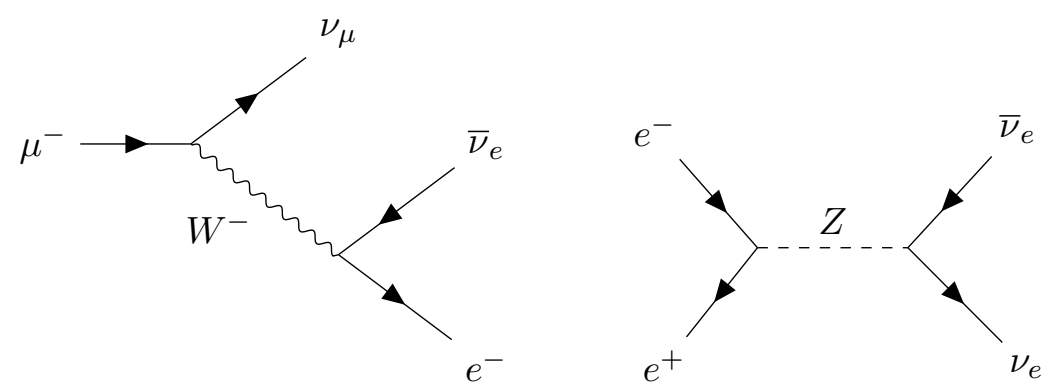

Figure 2.2.: Decay of muon into an electron (left) and neutrino/anti-neutrino production via a $\mathrm{Z}$ boson (right).

\subsubsection{Quantum Chromodynamics (QCD)}

The carrier of the strong force is the gluon, which is massless like the photon. The gluon only interacts with quarks, as they are the only fermions that carry a colour charge. Conceptually, this charge is similar to the electromagnetic charge, but there are three types of charge ("colours") instead of one, they are usually called red, blue and green. While quarks carry one of these colours, their anti-particle pendants carry an anti-colour, like anti-red. The gluon itself does carry a colour and an anti-colour, which is another difference to the photon which does not carry an electric charge itself.

The theory that describes the strong force and its interactions is fittingly called quantum chromodynamics (QCD), which is mathematically based on an $\mathrm{SU}(3)$ gauge group. As this is a non-abelian gauge group, the gluon can couple to itself. An example of this self-coupling can be seen in fig. 2.3.

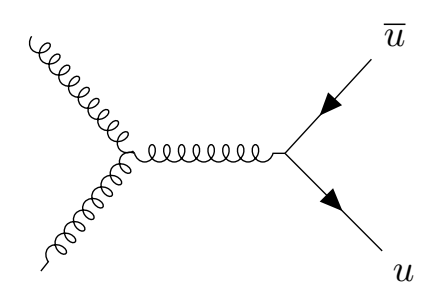

Figure 2.3.: Example of gluon-gluon self coupling. Two gluons fuse together and produce an up/anti-up quark pair.

\subsubsection{Higgs boson}

The last boson is the Higgs boson, which is special compared to the others. It is not connected to one of the three forces mentioned above and is the only boson that has a spin of 0. In 2012 it was discovered at the LHC [3] by the ATLAS [4] and CMS [5] collaborations. The theoretical prediction of the particle was made in the 1960s by Peter Higgs [6] and others [7,8]. He described a mechanism of spontaneous symmetry 
breaking for the electroweak interaction, that gives the $\mathrm{W}^{ \pm}$and $\mathrm{Z}$ bosons their masses. A consequence of this theory and the introduced Higgs field was the Higgs boson, which was found over 40 years later. By coupling to the Higgs also the fermions get their masses, which is called Yukawa coupling.

\begin{tabular}{llllrl}
\hline & & Symbol & EM charge & Mass $\left[\mathrm{GeV} / \mathrm{c}^{2}\right]$ & Spin \\
Force & Name & & & & \\
\hline Strong & Gluon & $\mathrm{g}$ & 0 & 0 & 1 \\
EM & Photon & $\gamma$ & 0 & 0 & 1 \\
\multirow{2}{*}{ Weak } & Z boson & $\mathrm{Z}$ & 0 & $91.1876(21)$ & 1 \\
& W boson & W & \pm 1 & $80.379(12)$ & 1 \\
\hline - & Higgs & H & 0 & $125.10(14)$ & 0 \\
\hline
\end{tabular}

Table 2.2.: Bosons in the standard model and their properties. Values for the masses taken from [2].

\subsection{CKM Matrix}

The weak interaction allows flavour changes via charged currents. In 1963, Cabibbo introduced a formalism to describe these interactions and explain why certain couplings in the quark sector were stronger than others using a $2 \times 2$ unitary matrix [9]. This model was later extended by Kobayashi and Maskawa to cover three quark generations [10] and is now called CKM matrix. The matrix connects the weak interaction states $q^{\prime}$ to the physical quark states $q$ :

$$
\left(\begin{array}{l}
d^{\prime} \\
s^{\prime} \\
b^{\prime}
\end{array}\right)=\left(\begin{array}{lll}
V_{u d} & V_{u s} & V_{u b} \\
V_{c d} & V_{c s} & V_{c b} \\
V_{t d} & V_{t s} & V_{t b}
\end{array}\right)\left(\begin{array}{c}
d \\
s \\
b
\end{array}\right)
$$

The size of the elements explains why interactions across generations are suppressed compared to interactions within a generation. A charged current transition of the form $u \rightarrow s$ involves the $V_{u s}$ element of the matrix for example. Assuming there are only three quark generations, the matrix has to be unitary. Mathematically this means $\sum_{i} V_{i j} V_{i k}^{*}=$ $\delta_{j k}$ and $\sum_{j} V_{i j} V_{k j}^{*}=\delta_{i k}$. Geometrically, these equations can be interpreted as triangles, the most frequently used one being

$$
V_{u d} V_{u b}^{*}+V_{c d} V_{c b}^{*}+V_{t d} V_{t b}^{*}=0 .
$$

The magnitudes of the elements are determined experimentally in various weak decays and interactions. The elements on the diagonal are close to $\approx 1$, the off-diagonal elements are significantly smaller, making cross-generation interactions less likely. The CKM matrix can be parametrized by three mixing angles $\left(\theta_{12}, \theta_{13}, \theta_{23}\right)$ and a complex phase 


\section{Introduction}

$\delta$ as [11]:

$$
V_{\mathrm{CKM}}=\left(\begin{array}{ccc}
c_{12} c_{13} & s_{12} c_{13} & s_{13} e^{-i \delta} \\
-s_{12} c_{23}-c_{12} s_{23} s_{13} e^{i \delta} & c_{12} c_{23}-s_{12} s_{23} s_{13} e^{i \delta} & s_{23} c_{13} \\
s_{12} s_{23}-c_{12} c_{23} s_{13} e^{i \delta} & -c_{12} s_{23}-s_{12} c_{23} s_{13} e^{i \delta} & c_{23} c_{13}
\end{array}\right)
$$

Here $s_{i j}=\sin \theta_{i j}$ and $c_{i j}=\cos \theta_{i j}$. Due to convenience another parametrisation, the Wolfenstein parametrisation [12], is used defining four new parameters $\lambda, A, \bar{\rho}$ and $\bar{\eta}$ as

$$
\begin{gathered}
s_{12}=\lambda=\frac{\left|V_{u s}\right|}{\sqrt{\left|V_{u d}\right|^{2}+\left|V_{u s}\right|^{2}}}, \quad s_{23}=A \lambda^{2}=\lambda\left|\frac{V_{c b}}{V_{u s}}\right| \\
s_{13} e^{i \delta}=V_{u b}^{*}=A \lambda^{3}(\rho+i \eta)=\frac{A \lambda^{3}(\bar{\rho}+i \bar{\eta}) \sqrt{1-A^{2} \lambda^{4}}}{\sqrt{1-\lambda^{2}}\left(1-A^{2} \lambda^{4}(\bar{\rho}+i \bar{\eta})\right)} .
\end{gathered}
$$

Using this parametrisation, the aforementioned unitarity triangle (see eq. (2.2)) can be drawn in the $\bar{\rho}-\bar{\eta}$ space and its vertices are $(0,0),(1,0)$ and $(\bar{\rho}, \bar{\eta})$. The angles of this triangle are then given as

$$
\begin{aligned}
& \beta=\phi_{1}=\arg \left(-\frac{V_{c d} V_{c b}^{*}}{V_{t d} V_{t b}^{*}}\right), \\
& \alpha=\phi_{2}=\arg \left(-\frac{V_{t d} V_{t b}^{*}}{V_{u d} V_{u b}^{*}}\right), \\
& \gamma=\phi_{3}=\arg \left(-\frac{V_{u d} V_{u b}^{*}}{V_{c d} V_{c b}^{*}}\right) .
\end{aligned}
$$

Experimental measurements allow to constrain the side lenghts and the angles of the triangle independently. Figure 2.4 shows a combination of all measurements performed and how they constrain the parameters of the triangle. 


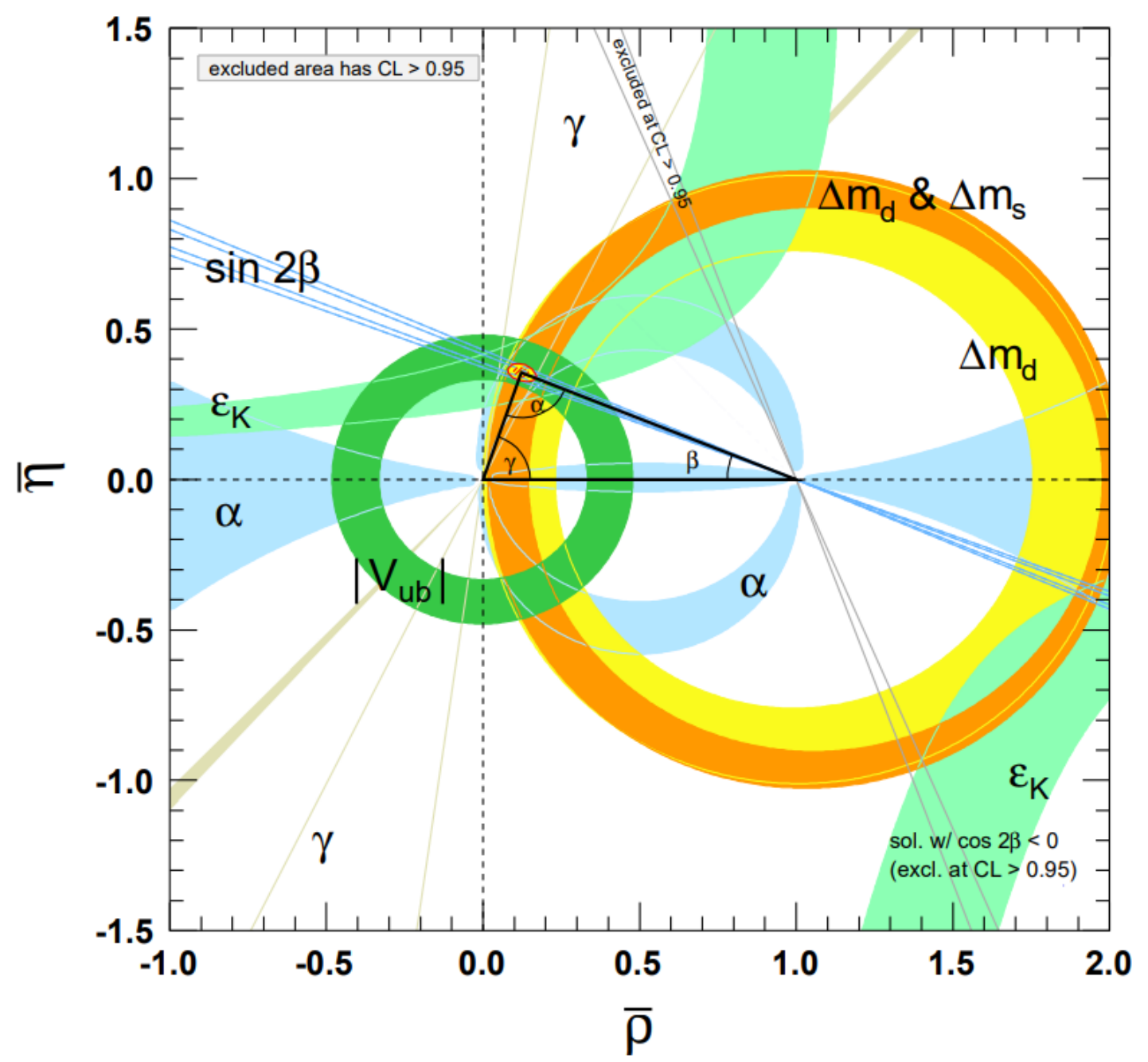

Figure 2.4.: Unitarity triangle with experimentally determined constraints on the angles and side lenghts. From [2]. 


\section{Introduction}

\subsection{Symmetries}

Ever since Emmy Noether formulated her theorem that connects symmetries to conservation laws (Noether Theorem [13]), symmetries have played an important role in physics in general and in particular in particle physics. While the Noether theorem is connected to continuous symmetries, particle physics also uses discrete symmetries.

Parity: The quantum mechanical parity operator $P$ changes the sign of one of the spatial dimensions, effectively "mirroring" the system.

$$
\overrightarrow{\mathbf{x}} \rightarrow-\overrightarrow{\mathbf{x}}
$$

Depending on their eigenvalue, particles are classified as scalars or axial-vectors $(\mathrm{P}=+1)$ or vectors or pseudo-scalars $(\mathrm{P}=-1)$. Historically, it was assumed that mirror images of physical processes behave exactly the same as the original ones, which means that parity is conserved. However, an experiment by C.S. Wu [14], in which she measured the spin orientation of neutrinos from a beta decay, showed that parity is violated maximally in weak interactions.

Charge Conjugation: The charge conjugation operator $C$ converts any particle to its anti-particle. In this process all internal (additive) quantum numbers like electric charge are conjugated. Properties like mass and spin are not affected by this transformation.

Time Reversal: The last operator is the time reversal operator $T$, which changes the direction of time in a system.

$$
t \rightarrow-t
$$

Test of its conservation are more difficult than for the other two, but there are reasons to believe that it is also violated (see next section).

\subsection{CP Violation}

While parity as well as charge conjugation are violated in a maximal way in weak interactions, the combination $C P$ is not. Until 1964 this symmetry seemed to be conserved exactly, but then Cronin and Fitch demonstrated that even this symmetry was broken [15] in the Kaon system. The measured effect was very small though. In $2001 \mathrm{CP}$ violation was also observed in the $B$ meson system by the BaBar [16] and Belle [17] collaborations. There are three types of $\mathrm{CP}$ violation $(\mathrm{CPV})$ :

Direct: In general the decay amplitudes of a given particle $M$ and its CP counterpart $\bar{M}$ to a final state $f / \bar{f}$ are given as

$$
\begin{array}{ll}
A_{f}=\langle f|H| M\rangle, & \bar{A}_{f}=\langle f|H| \bar{M}\rangle \\
A_{\bar{f}}=\langle\bar{f}|H| M\rangle, & \bar{A}_{\bar{f}}=\langle\bar{f}|H| \bar{M}\rangle
\end{array}
$$


Here $H$ denotes the Hamiltonian of the weak interaction. Direct $\mathrm{CP}$ violation means that the decay of a particle $M$ to a specified final state $f$ has lower/higher probability than its $\mathrm{CP}$ counterpart.

$$
\begin{gathered}
\left|\bar{A}_{\bar{f}} / A_{f}\right| \neq 1 \\
\Rightarrow \Gamma(M \rightarrow f) \neq \Gamma(\bar{M} \rightarrow \bar{f})
\end{gathered}
$$

For charged mesons direct $\mathrm{CP}$ violation is the only possible source of $\mathrm{CP}$ violation. The asymmetry in these kind of decays is usually defined as

$$
\mathcal{A}_{f^{ \pm}}=\frac{\Gamma\left(M^{-} \rightarrow f^{-}\right)-\Gamma\left(M^{+} \rightarrow f^{+}\right)}{\Gamma\left(M^{-} \rightarrow f^{-}\right)+\Gamma\left(M^{+} \rightarrow f^{+}\right)}
$$

The effect of this kind of CPV is rather small, but was measured in experiments, one example is the decay $B^{0} \rightarrow \pi^{-} K^{+}[18]$.

Mixing: Neutral mesons can show the effect of $\mathrm{CP}$ violation in the mixing of flavour eigenstates. Mixing describes the phenomenon when a meson $M^{0}$ undergoes a transformation to its $\mathrm{CP}$ counterpart $\bar{M}^{0}$, which can be described with a Feynman box-diagram:

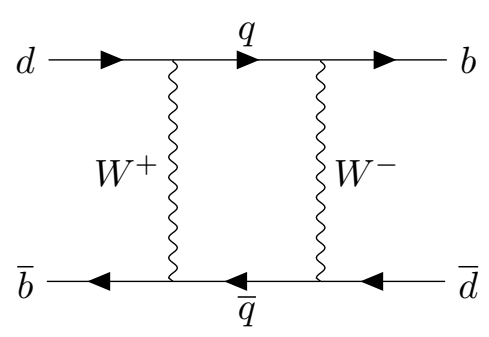

The time evolution of this mixing process is given by a $2 \times 2$ Hamiltonian $\mathbf{H}$ :

$$
\mathbf{H}=\mathbf{M}-\frac{i}{2} \boldsymbol{\Gamma}
$$

Here the off-diagonal elements describe flavour changing transitions $M^{0} \leftrightarrow \bar{M}^{0}$. The eigenvectors of the Hamiltonian are the mass eigenstates $M_{L / H}$ of the mesons as they have a defined mass and decay width. They are linked to the flavour eigenstates $M^{0} / \bar{M}^{0}$ by

$$
\begin{aligned}
\left|M_{L}\right\rangle & \propto p \sqrt{1-z}\left|M^{0}\right\rangle+q \sqrt{1+z}\left|\bar{M}^{0}\right\rangle, \\
\left|M_{H}\right\rangle & \propto p \sqrt{1+z}\left|M^{0}\right\rangle-q \sqrt{1-z}\left|\bar{M}^{0}\right\rangle .
\end{aligned}
$$




\section{Introduction}

The complex parameters $p, q$ and $z$ introduced here are linked to the Hamiltonian by

$$
\begin{aligned}
\left(\frac{q}{p}\right)^{2} & =\frac{\mathbf{M}_{12}^{*}-\frac{i}{2} \boldsymbol{\Gamma}_{12}^{*}}{\mathbf{M}_{12}-\frac{i}{2} \boldsymbol{\Gamma}_{12}}, \\
z & =\frac{\left(\mathbf{M}_{11}-\mathbf{M}_{22}\right)-\frac{i}{2}\left(\boldsymbol{\Gamma}_{11}-\boldsymbol{\Gamma}_{22}\right)}{\Delta m-\frac{i}{2} \Delta \Gamma},
\end{aligned}
$$

where $\Delta M$ and $\Delta \Gamma$ are the mass and decay width differences between the two mass eigenstates. CP violation in the mixing occurs when $|q / p| \neq 1$. Considering a semileptonic particle decay of the form $M / \bar{M} \rightarrow \ell^{ \pm} X$, the asymmetry due to the mixing is defined as

$$
\begin{aligned}
\mathcal{A}_{S L}(t) & =\frac{\frac{\mathrm{d} \Gamma}{\mathrm{d} t}\left[\bar{M}_{\mathrm{phys}}^{0}(t) \rightarrow \ell^{+} X\right]-\frac{\mathrm{d} \Gamma}{\mathrm{d} t}\left[M_{\mathrm{phys}}^{0}(t) \rightarrow \ell^{-} X\right]}{\frac{\mathrm{d} \Gamma}{\mathrm{d} t}\left[\bar{M}_{\mathrm{phys}}^{0}(t) \rightarrow \ell^{+} X\right]+\frac{\mathrm{d} \Gamma}{\mathrm{d} t}\left[M_{\mathrm{phys}}^{0}(t) \rightarrow \ell^{-} X\right]} \\
& =\frac{1-|q / p|^{4}}{1+|q / p|^{4}}
\end{aligned}
$$

Interference: The type of CPV with the largest effect (for B mesons) can be observed in systems where a meson and the corresponding anti-meson can decay to the same $\mathrm{CP}$ eigenstate

$$
\begin{aligned}
& M^{0} \rightarrow f_{C P} \\
& M^{0} \rightarrow \bar{M}^{0} \rightarrow f_{C P} .
\end{aligned}
$$

$\mathrm{CP}$ violation of this type occures when

$$
\operatorname{J} m\left(\lambda_{f_{C P}}\right)=\operatorname{J} m\left(\frac{q}{p} \frac{\bar{A}_{f}}{A_{f}}\right) \neq 0
$$

The asymmetry in this case is defined as

$$
\mathcal{A}_{f_{C P}}(t)=\frac{\frac{\mathrm{d} \Gamma}{\mathrm{d} t}\left[\bar{M}_{\mathrm{phys}}^{0}(t) \rightarrow f_{C P}\right]-\frac{\mathrm{d} \Gamma}{\mathrm{d} t}\left[M_{\mathrm{phys}}^{0}(t) \rightarrow f_{C P}\right]}{\frac{\mathrm{d} \Gamma}{\mathrm{d} t}\left[\bar{M}_{\mathrm{phys}}^{0}(t) \rightarrow f_{C P}\right]+\frac{\mathrm{d} \Gamma}{\mathrm{d} t}\left[M_{\mathrm{phys}}^{0}(t) \rightarrow f_{C P}\right]}
$$

A prominent example for this type of CPV is the decay of a neutral B meson $\left(B^{0}\right)$ to a $J / \Psi$ and $K_{s}$. For this particular decay eq. (2.15) can be expressed as

$$
\mathcal{A}_{J / \Psi K_{s}}(t)=-\xi_{f} \sin (2 \beta) \sin (\Delta m \Delta t),
$$

where $\xi_{f}$ is the CP eigenvalue of the final state and $\beta=\arg \left(-\frac{V_{c d} V_{c b}^{*}}{V_{t d} V_{t b}^{*}}\right)$ one of the angles from the unitarity triangle. To measure this asymmetry it is necessary to perform a time-dependent analysis as eq. (2.16) shows. 


\subsection{B-Physics and B-Factories}

The study of mesons with bottom quarks is referred to as b-physics. The bottom quark is the heaviest quark that hadronizes (forms bound states before it decays), which makes it particularly interesting together with its long life time. To study b-physics the BaBar [19] and the Belle [20] experiment were constructed. The accelerators are referred to as B-Factories because they are operated at an energy level where almost exclusively Bmeson pairs $\left(B^{0} / \bar{B}^{0}\right.$ and $\left.B^{+} / B^{-}\right)$are produced. In contrast to accelerators like the LHC, where protons and anti-protons are collided at an energy of $14 \mathrm{TeV}$, they were not designed to search for physics at the high energy frontier but to perform high precision measurements.

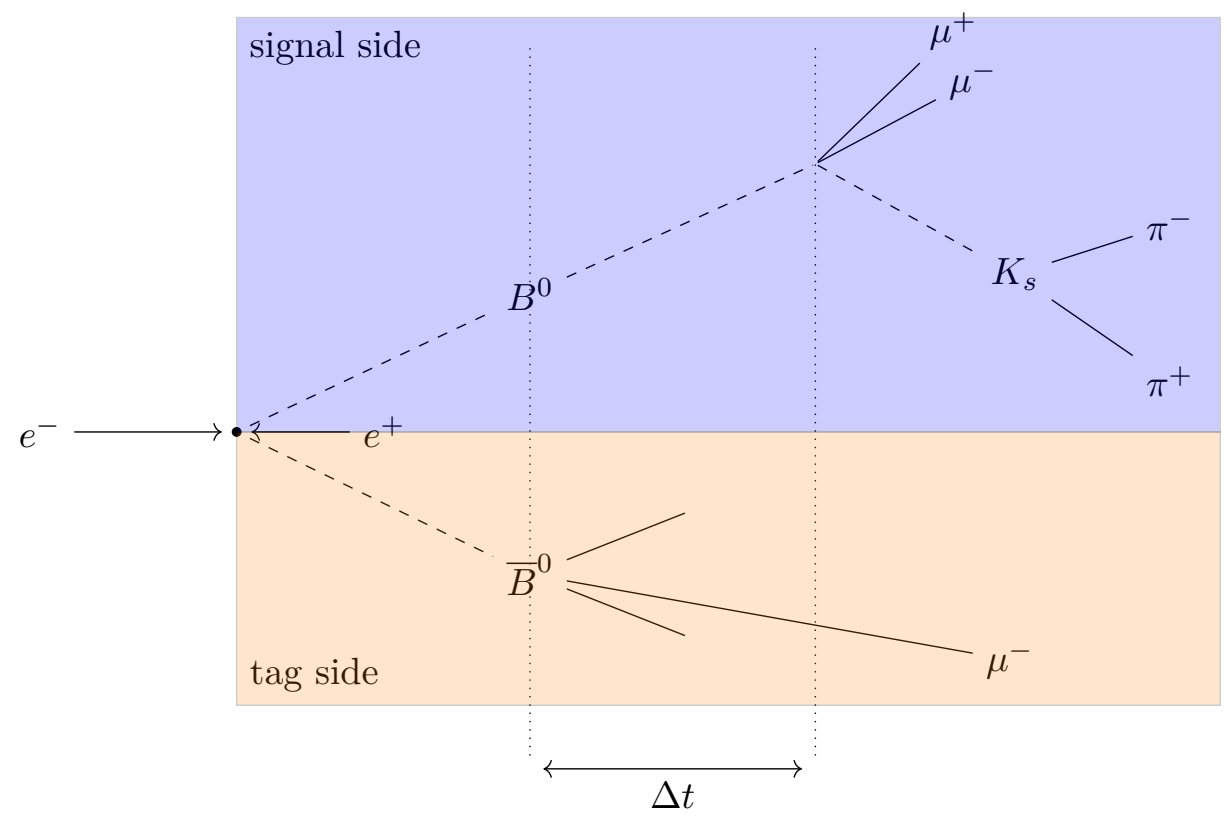

Figure 2.5.: Sketch of the measurement technique used by Belle for the $B^{0} \rightarrow J / \Psi K_{s}$ decay. One of the $\mathrm{B}$ mesons decays into the desired signal mode, while the flavour of the other B meson is tagged by the charge of a lepton in a semi-leptonic decay mode.

Their main purpose was the measurement of $\mathrm{CP}$ violation in the neutral $\mathrm{B}$ meson system and the precise measurement of the CKM matrix elements $V_{u b}$ and $V_{c b}$. As mentioned in section 2.4, the $\mathrm{CP}$ violation in interference of mixing and decay has the largest measurable effect in the B system. Because of the accelerator and detector design, Belle was able to make a time dependent measurement of CPV in the $B^{0} \rightarrow J / \Psi K_{s}$ decay. As the KEKB accelerator, at which the Belle experiment was operating, was an asymmetric $e^{+} / e^{-}$collider, the created $B^{0} / \bar{B}^{0}$ pairs were boosted in one direction, which allowed the experimentalists to translate spacial distances along the beam axis 


\section{Introduction}

$\Delta z$ into time differences via

$$
\Delta t=\frac{\Delta z}{\beta \gamma c},
$$

where $\beta \gamma=0.425$ is the Lorentz boost of the system. To make this kind of measurement possible, a high resolution vertex detector is required to determine the positions of the decay vertices precisely and measure $\Delta z$. Furthermore, the entanglement of the $B^{0} / \bar{B}^{0}$ pair is exploited. Both $B$ mesons are reconstructed and the flavour of the $B$ meson that does not decay to the $J / \Psi K_{s}$ mode is determined by semileptonic decay modes $^{1}$, fixing the flavour of the signal-side $B$ meson. The positions of the decay vertices of both mesons are then compared and the time difference is calculated. This allows to perform the desired time-dependent analysis required to determine the $\mathrm{CP}$ asymmetry, see eq. (2.16). A sketch of this specific measurement technique can be seen in fig. 2.5.

Because of the large number of produced B mesons, data from the B-Factories was also used to determine the magnitude of various CKM matrix elements. The value for $\left|V_{u b}\right|$ can be extracted from $B \rightarrow X_{u} \ell \bar{\nu}$ decays. Semileptonic decays of the type $B \rightarrow D^{(*)} \ell \bar{\nu}$ on the other hand can be used to determine $\left|V_{c b}\right|$. Together with QCD models and calculations data from the B-Factories can also be used to constrain the values of other elements like $V_{t d}$ and $V_{t s}$ by measuring $B-\bar{B}$ oscillations.

While B-Factories play a significant role in the field of B-physics, B mesons are also created and analysed at other accelerators. The LHCb detector [21] at the LHC is an example of an experiment that studies the physics of b-quarks at a hadron collider. In contrast to Belle and BaBar it is not a classical $4 \pi$ detector, but a single-arm spectrometer. While the initial four-momentum state in proton/anti-proton collisions is not completely known in contrast to electron/positron collisions, the LHCb experiment was still able to perform a variety of measurements that were done by Belle and BaBar before and provides another angle on b-physics.

When searching for new physics beyond the standard model B-Factories are interesting as well. While CP violation in the quark sector has been observed for many years now, the current models and observations cannot explain the vast matter/anti-matter asymmetry observed in the universe, which is why physicists are looking for additional sources of $\mathrm{CP}$ violation.

In the standard model the coupling of $W^{ \pm}$bosons to leptons is assumed to be universal, e.g. there is no difference between an electron or a tau. At Belle this universality was tested by comparing the branching rations of $B \rightarrow D^{(*)} \ell \nu_{\ell}$ and $B \rightarrow D^{(*)} \tau \nu_{\tau}$ decays

$$
\begin{aligned}
R(D) & =\frac{\mathcal{B}\left(B \rightarrow D \tau \nu_{\tau}\right)}{\mathcal{B}\left(B \rightarrow D \ell \nu_{\ell}\right)} \\
R\left(D^{*}\right) & =\frac{\mathcal{B}\left(B \rightarrow D^{*} \tau \nu_{\tau}\right)}{\mathcal{B}\left(B \rightarrow D^{*} \ell \nu_{\ell}\right)},
\end{aligned}
$$

\footnotetext{
${ }^{1}$ Other decay modes are also possible, but experimentally more difficult.
} 

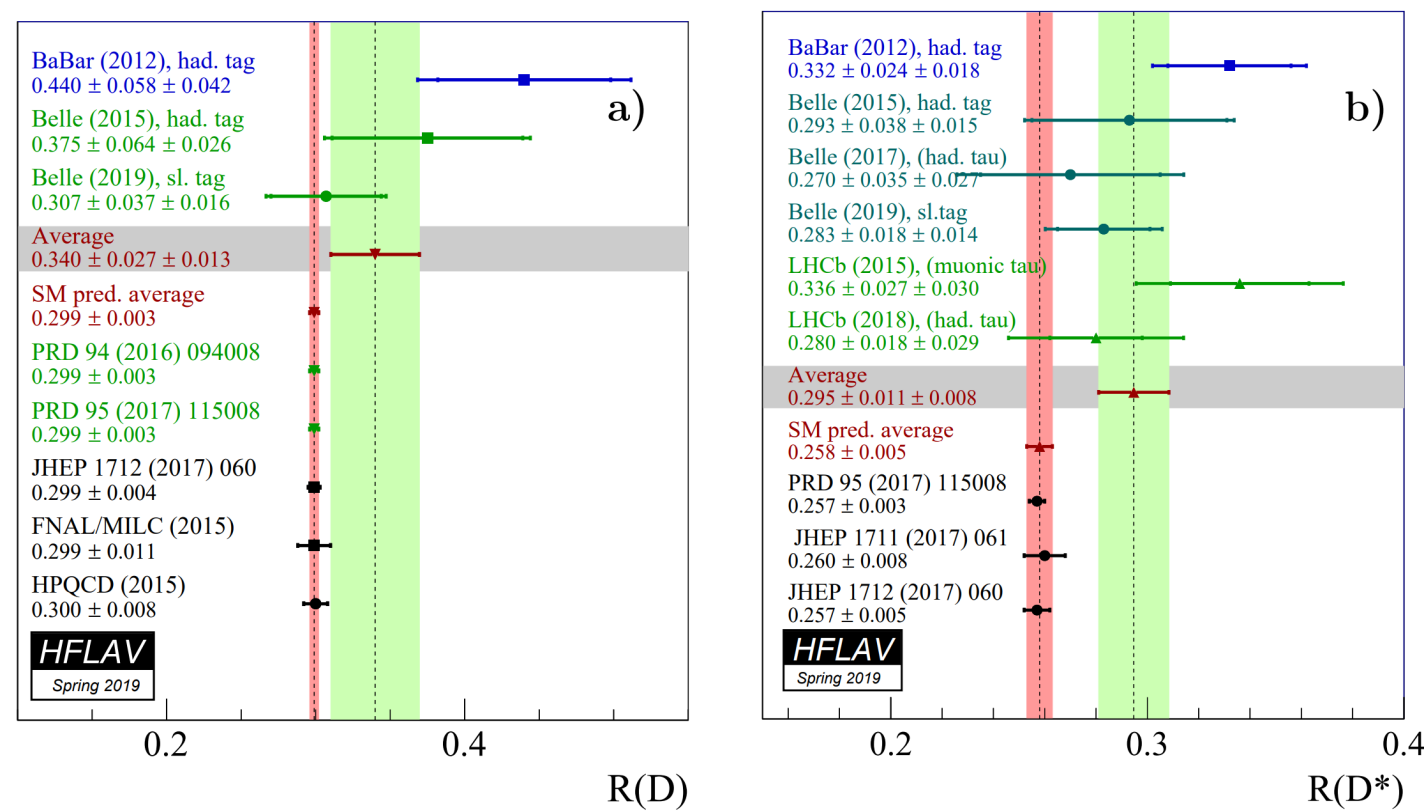

Figure 2.6.: Measurements of $R(D)$ (left) and $R\left(D^{*}\right)$ (right). From [22].

where $\ell$ is either an electron or a muon. This ratio has also been measured at BaBar and $\mathrm{LHCb}$. All measurements show a deviation from the standard model prediction. A summary of the individual measurements performed by the various experiments and the current world average can be seen in fig. 2.6. The averages for $R(D)$ and $R\left(D^{*}\right)$ exceed the standard model expectation by $1.4 \sigma$ and $2.5 \sigma$, respectively [22]. The deviations from the standard model prediction might hint at new physics processes like a charged Higgs boson that couples more strongly to the tau than to the other leptons, thereby influencing the branching ratio and the $R$ value.

Another example of new physics might lie in possible lepton flavour violation. While the standard model forbids processes like $\tau \rightarrow \ell \gamma$, there are various extensions of the standard model that would allow such processes. The Belle and BaBar experiment have set upper limits on the branching ratio of the corresponding processes in the past. Nevertheless, these processes might be possible.

Because of the reasons listed above and many more, the Belle II experiment was planned as a successor to the Belle experiment. The following chapter will give an indepth look at this new detector and the new accelerator built to study b-physics further. Are more comprehensive list of the physics that will be studied at Belle II can be found in the Belle II physics book [23]. 

CHAPTER 3

\section{SuperKEKB and Belle 2}

This chapter gives an overview of the SuperKEKB accelerator, its particularities and the basic concepts of particle accelerators in general. The concepts of beam backgrounds are also introduced as they are important for the analyses presented in chapter 6 . In addition, the chapter describes Belle II with its various subsystems. This also includes the vertex detector with its pixel detector. The description is limited to the mechanics and geometry of the pixel detector. Details about its technology are given in chapter 4 .

\subsection{SuperKEKB}

The SuperKEKB [24] accelerator is the successor to the KEKB [25] accelerator and is located at the KEK research centre in Tsukuba, Japan. Like its predecessor, it is an asymmetric electron-positron collider with a centre-of-mass energy of $10.58 \mathrm{GeV}$, which corresponds to the $\Upsilon(4 S)$ resonance. The $\Upsilon(4 S)$ is a bound $b \bar{b}$ state that decays almost exclusively into pairs of $\mathrm{B}$ mesons $\left(48.6 \% B^{0} / \bar{B}^{0}\right.$ and $\left.51.4 \% B^{+} / B^{-}[2]\right)$. The electron beam has an energy of $7.007 \mathrm{GeV}$ and the positron beam an energy of $4 \mathrm{GeV}$. Because of the energy difference, the accelerator beams/rings are also referred to as high- and low-energy ring ( $H E R$ and $L E R$ ). The most important consequence of the difference in energy is that the centre-of-mass system is boosted into the direction of the electron beam. As mentioned before, this boost is desired to make time dependent measurements possible. Figure 3.1 shows a sketch of the accelerator together with the injection linac (linear accelerator).

Compared to KEKB, the energy of the positron ring was increased slightly while the electron beam energy was decreased. The motivation of this change is a reduction of beam backgrounds [26]. The relativistic boost of the $B / \bar{B}$ system was therefore lowered from $\beta \gamma \approx 0.42$ to $\approx 0.28$. As a consequence the average flight length of a $B$ is decreased 


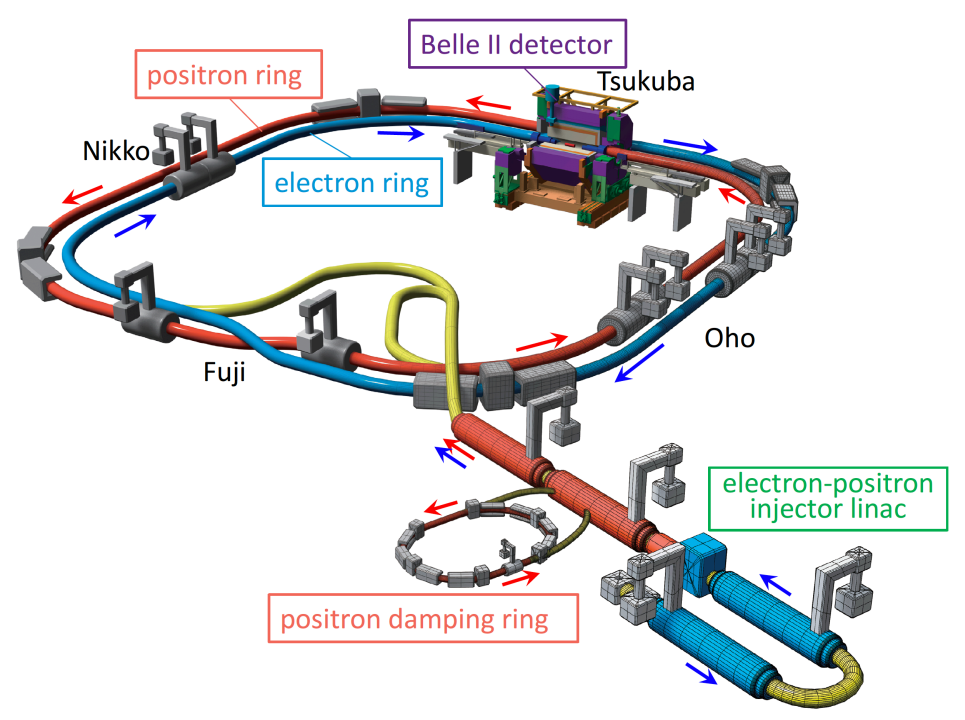

Figure 3.1.: Overview of the SuperKEKB accelerator, together with the pre-accelerators. The Belle II detector is located in the Tsukuba hall, the other three halls (Nikko, Fuji and Oho) are currently not used [26].

from $\approx 200 \mu \mathrm{m}$ to $\approx 130 \mu \mathrm{m}$. To compensate for this effect a vertex detector with a higher resolution compared to Belle is required.

Like other synchrotron type colliders, SuperKEKB accelerates bunches of particles in its rings. Within each bunch are a large number of electrons or positrons $\left(\approx 10^{10}\right)$. The design foresees 2500 bunches to circulate at the same time. Through focusing magnets these bunches are eventually brought to collisions at the interaction point. During these bunch crossings not all particles of the bunches interact. The particles that do are naturally removed from the bunch, decreasing the number of particles in the ring over time. To measure the rate at which particles collide at any particle collider, the concept of luminosity $\mathcal{L}$ is used, it is defined as

$$
\mathcal{L}=\frac{1}{\sigma} \frac{\mathrm{d} N}{\mathrm{~d} t}
$$

where $\sigma$ is the cross-section of the collision process and $\mathrm{d} N / \mathrm{d} t$ the number of collisions per second. By integrating over a given time-span the integrated luminosity $\mathcal{L}_{\text {int }}$ can be calculated as

$$
\mathcal{L}_{\text {int }}=\int \mathcal{L} \mathrm{d} t
$$

which is a measure for the collected data-sample of a collider. The goal of SuperKEKB is to achieve a luminosity of $8 \times 10^{35} \mathrm{~cm}^{2} \mathrm{~s}^{-1}$, which is 40 times higher than what KEKB has achieved. The target for the integrated luminosity is $50 \mathrm{ab}^{-1}$. 
To achieve a higher luminosity, it was decided to not simply increase the beam currents $I_{ \pm}$but change the collision scheme. The luminosity $\mathcal{L}$ depends on several geometrical and accelerator related variables like the beam currents $I_{ \pm}$, the beam sizes at the interaction point $\sigma_{x, y}^{*}$, the vertical beta function at the interaction point $\beta_{y}^{*}$ and beam-beam tune-shift parameters $\xi_{y \pm}^{*}$. For SuperKEKB the luminosity is given as [24]:

$$
\mathcal{L}=\frac{\gamma_{ \pm}}{2 e r_{e}}\left(1+\frac{\sigma_{y}^{*}}{\sigma_{x}^{*}}\right) \frac{I_{ \pm} \xi_{y \pm}^{*}}{\beta_{y}^{*}} \frac{R_{L}}{R_{\xi_{y}}}
$$

Here $\gamma_{ \pm}$are the Lorentz factors of the electron and positron beam and $r_{e}$ the classical electron radius. The vertical beta function is related to the vertical beam size $\sigma_{y}^{*}$. $R_{L} / R_{\xi_{y}} \approx 0.8$ is the ratio of the reduction factors for the luminosity and the beam-beam parameter [27]. For SuperKEKB the vertical beam size is reduced to $62 / 48 \mathrm{~nm}$ for the electron and positron ring, respectively. The crossing angle of the bunches is changed from $22 \mathrm{mrad}$ to $84 \mathrm{mrad}$. A visualisation of this so-called nanobeam [28] scheme is shown in fig. 3.2. At Belle, the beams collided head-on, meaning that the interaction region was roughly the length of the bunches. In the nano beam scheme the longitudinal size of the overlap is reduced significantly which allows to reduce the vertical beta function and still avoid the hourglass effect. Compared to Belle, the vertical beta function is decreased by a factor of 20 . Together with a factor two increase of the beam currents this gives the desired 40 times increase in luminosity [26]. The actions taken to increase the luminosity do however come at the cost of increased backgrounds.

\section{Head on collison}

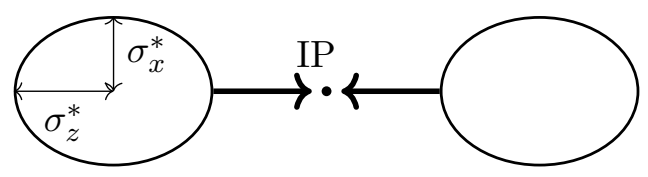

\section{Nano beam scheme}

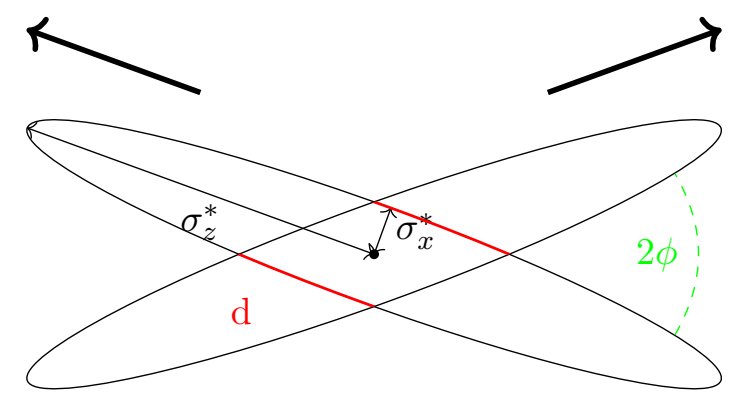

Figure 3.2: Comparison of Head on collision scheme as used in Belle (left) and the new nano beam scheme used in Belle II (right). For the nano beam scheme the longitudinal size of the overlap $d=\sigma_{x}^{*} / \phi$ is significantly smaller than the bunch size, allowing for a smaller $\beta_{y}^{*}$.

\subsubsection{Backgrounds}

Every particle physics experiment has to deal with undesirable backgrounds. There are various sources for these backgrounds and they can be classified in two categories: luminosity depended ones and machine related ones. The former ones are only present when 


\section{SuperKEKB and Belle2}

the beams collide, while the latter originate from the beams themselves. Backgrounds are problematic for two reasons, firstly they hide potential signal events in the detector and secondly damage the detector.

\section{Touschek scattering}

Touschek scattering [29] describes the effect of intra-bunch coulomb scattering. The particles within a bunch interact with each other due to the coulomb force and a large single scattering can kick a particle out of the bunch. The interaction between the particles can also modify their trajectory by transferring transverse momenta into longitudinal momenta. This will lead to a loss of the particle if it is out of the acceptance of the accelerator momentum space. The loss of particles naturally decreases the lifetime of the beam. When a particle that was removed from the bunch hits the inner wall of the beam pipe, this leads to the production of a shower of particles. If this happens close enough to the interaction point, the particles from this shower will hit the detector which is then called Touschek background.

As pointed out before, SuperKEKB uses the nano beam scheme for its bunches to increase the luminosity. This scheme does however increase the Touschek effect significantly compared to KEKB, as the particles within a bunch are closer together. The most accurate description of the Touschek scattering rate $R$ is given by the Piwinski formula [30].

$$
R_{\text {Touschek }}^{\text {Piwinsi }}=\frac{r_{e}^{2} c \beta_{x} \beta_{y} \sigma_{h} N^{2}}{8 \sqrt{\pi} \beta^{2} \gamma^{4} \sigma_{x \beta}^{2} \sigma_{y \beta}^{2} \sigma_{s} \sigma_{p}} F\left(\tau_{m}, B_{1}, B_{2}\right)
$$

with

$$
\begin{aligned}
& F=\int_{\tau_{m}}^{\infty} \mathrm{d} \tau e^{-B_{1} \tau} I_{0}\left(B_{2} \tau\right) \frac{\sqrt{\tau}}{\sqrt{1+\tau}}\left(\left(2+\tau^{-1}\right)^{2}\left(\frac{\tau \tau_{m}^{-1}}{1+\tau}-1\right)+1\right. \\
&\left.\quad-\frac{\sqrt{1+\tau}}{\sqrt{\tau \tau_{m}^{-1}}}-\frac{4 \tau+1}{2 \tau^{2}} \ln \frac{\tau \tau_{m}^{-1}}{1+\tau}\right) . \\
& B_{1}=\frac{1}{2 \beta^{2} \gamma^{2}}\left(\frac{\beta_{x}^{2}}{\sigma_{x \beta}^{2}}-\frac{\beta_{x}^{2} \sigma_{h}^{2} \tilde{D}_{x}^{2}}{\sigma_{x \beta}^{4}} \frac{\beta_{y}^{2}}{\sigma_{y \beta}^{2}}-\frac{\beta_{y}^{2} \sigma_{h}^{2} \tilde{D}_{y}^{2}}{\sigma_{y \beta}^{4}}\right), \\
& B_{2}^{2}=B_{1}^{2}-\frac{\beta_{x}^{2} \beta_{y}^{2} \sigma_{h}^{2}}{\beta^{4} \gamma^{4} \sigma_{x \beta}^{4} \sigma_{y \beta}^{4} \sigma_{p}^{2}}\left(\sigma_{x}^{2} \sigma_{y}^{2}-\sigma_{p}^{4} D_{x}^{2} D_{y}^{2}\right), \\
& \tau_{m}=\beta^{2} \delta_{m}^{2}=\beta^{2}\left(\frac{\Delta p_{m}}{p}\right)^{2} \\
& \sigma_{h}= \frac{\sigma_{x \beta} \sigma_{y \beta} \sigma_{p}}{\sqrt{\tilde{\sigma}_{x}^{2} \sigma_{y \beta}^{2}+\tilde{\sigma}_{y}^{2} \sigma_{x \beta}^{2}-\sigma_{x \beta}^{2} \sigma_{y \beta}^{2}}} \\
& \tilde{D}_{x, y}=\alpha_{x, y} D_{x, y}+\beta_{x, y} D_{x, y}^{\prime}, \\
& \tilde{\sigma}_{x, y}^{2}=\sigma_{x \beta, y \beta}^{2}+\sigma_{p}^{2}\left(D_{x, y}^{2}+\tilde{D}_{x, y}^{2}\right)
\end{aligned}
$$


Here $N_{0}$ is the number of particles in a bunch and $r_{e}$ the classical electron radius. $I_{0}$ is the modified Bessel function while $\beta$ and $\gamma$ are the Lorentz factors. The beam sizes are $\sigma_{x, y, s}$ and $\sigma_{x \beta, y \beta}$ (without momentum spread). $D_{x, y}, D_{x, y}^{\prime}, \alpha_{x, y}$ and $\beta_{x, y}$ are the optical functions of the accelerator. From eq. (3.2) one can see that the scattering rate increases quadratically with the number of particles per bunch. As the number of bunches is usually fixed this corresponds to a quadratic increases with the beam current $I$. Using a non-relativistic and flat-beam approximation of Piwinski's formula as described in [24], it can be seen that the scattering rate is proportional to $\gamma^{-3}$ and therefore scales with the beam energy like $E^{-3}$. As a consequence the Touschek background is more pronounced in the positron ring. For the analyses presented in this paper a simplified and more heuristic description of the scattering rate $R_{\text {Touschek }}$ is chosen.

$$
R_{\text {Touschek }}=T \frac{I^{2}}{\sigma n_{b}}
$$

where $T$ is a free "Touschek" parameter for the scattering rate, $I$ the beam current, $n_{b}$ the number of bunches and $\sigma$ the vertical bunch size. The aforementioned energy dependency is absorbed into the Touschek parameter as the energy during normal operation is constant at SuperKEKB. To mitigate the effect of Touschek scattering, SuperKEKB uses multiple collimators around the ring. These collimator block particles that deviate too far from their nominal trajectory. They are also movable to some degree to adjust them in an optimal way during the operation of the accelerator.

\section{Beam-gas}

Another machine related background is the beam-gas scattering. It refers to the interaction of particles from the bunches with atoms of the gas in the beam. While there is a vacuum inside the beam pipe, the gas "desorption" effect [31] releases gas molecules from the beam pipe which can then interact with the particles of the beam. The beamgas scattering can be subdivided into coulomb scattering and bremsstrahlung scattering. The former can lead to a change of the trajectory of a particle from the bunch. When this particle hits the beam beam pipe, a shower is created similar to the Touschek scattering. Because of the smaller beam pipe of SuperKEKB compared to its predecessor $(1 \mathrm{~cm}$ vs $1.5 \mathrm{~cm}$ ), the effect is more pronounced than before. The beam-gas bremsstrahlung, in which the particle's energy is lowered, is a lesser concern as it can be suppressed effectively with the installed collimators. A simplified, heuristic description of the total scattering rate $R_{B}$ for beam-gas interactions is given by

$$
R_{B}=B I P Z_{\text {eff }}^{2}
$$

where $B$ is the beam-gas parameter, $P$ the vacuum pressure in the beam pipe and $Z_{\text {eff }}$ the effective atomic number of the gas in the beam pipe. The collimators used for the reduction of the Touschek background reduce the beam-gas background as well, but they need to have a minimum width. If they are too narrow, they cause Transverse Mode Coupling (TMC) instabilities [32]. 


\section{SuperKEKB and Belle2}

\section{Synchrotron radiation}

Similar to other accelerators, another background is synchrotron radiation (SR). Created by the constant acceleration of the particles required to keep them on their trajectory around the ring, the photons of the SR can be a direct threat to the detector. In general, the power of the synchrotron radiation scales with the energy of the beam currents like $E^{4}$. In order to prevent that these photons hit the vertex detector directly, which could cause significant damage, there are special structures around the beam pipe to stop them before they can reach it.

As a second counter measure the beam pipe is coated with a gold layer to absorb the photons. The energy of the photons is in the keV range. In general, the power of the SR is proportional to the beam energy and the strength of the magnetic field, therefore the SR from the HER is the dominant component.

\section{Injection Background}

SuperKEKB uses a "continuous injection" scheme where new bunches are injected into the electron and positron ring with a repetition rate of up to $50 \mathrm{~Hz}$. The injection of new bunches is done via kicker magnets and a septum magnet. After the injection the bunches start to perform a Betatron-oscillation around the nominal beam orbit. This oscillation is damped by synchrotron radiation and a designated bunch-by-bunch feedback system until the new bunch reaches the nominal orbit and merges with the "old" bunch. This mechanism is called betatron phase space injection [24].

During the oscillation these bunches lead to increased background level, which is why they are also referred to as noisy bunches. Because this effect is temporary, a trigger veto signal is used that notifies the Belle II subsystems about these bunches so that the systems can take actions to deal with the increased background level.

\section{Radiative Bhabha scattering}

One of the luminosity dependent backgrounds is Radiatitive Bhabha scattering

$$
e^{+} e^{-} \rightarrow e^{+} e^{-} \gamma
$$

in which an additional photon is created. Figure 3.3 shows the corresponding Feynman diagrams for this particular process. While the photons represent a background themselves for the detector, they can also produce neutrons by interacting with the magnets of SuperKEKB. As the scattered particles lose energy because of the radiated photon, the focusing magnets can "overbend" the particle, which is then lost, hits the beam pipe and creates an electromagnetic shower. To reduce this effect, different focusing magnets for the incoming and outgoing beams are used. 

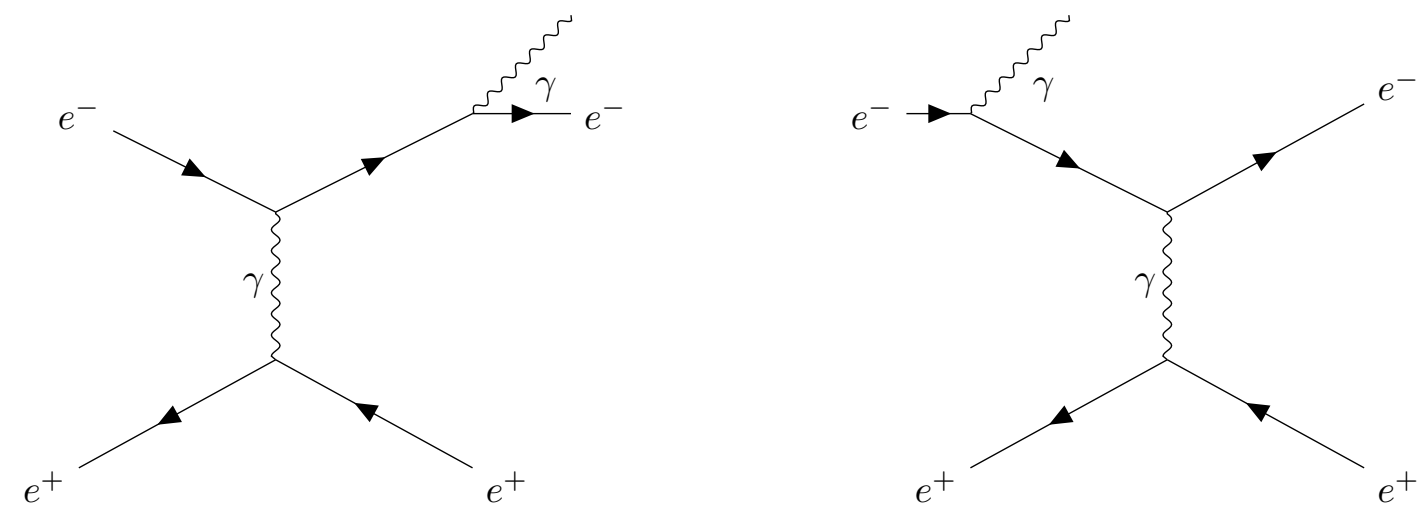

Figure 3.3.: Radiative Bhabha scattering with an additional photon at the end of the process.

\section{Two-photon interaction}

Another luminosity dependent background is the QED process

$$
e^{+} e^{-} \rightarrow e^{+} e^{-} f \bar{f}
$$

with four fermions in the final state, created by a two photon interaction as depicted in fig. 3.4. The fermion/antifermion pair (mostly electron/positron) is very low energetic and characterised by a small transverse momentum $p_{t}$. For the innermost detector part it is an important background since the low momentum particles tend to curl in the magnetic field and give multiple hits in the detector. Because of the smaller beam pipe, this is also a background that is larger than at KEKB.

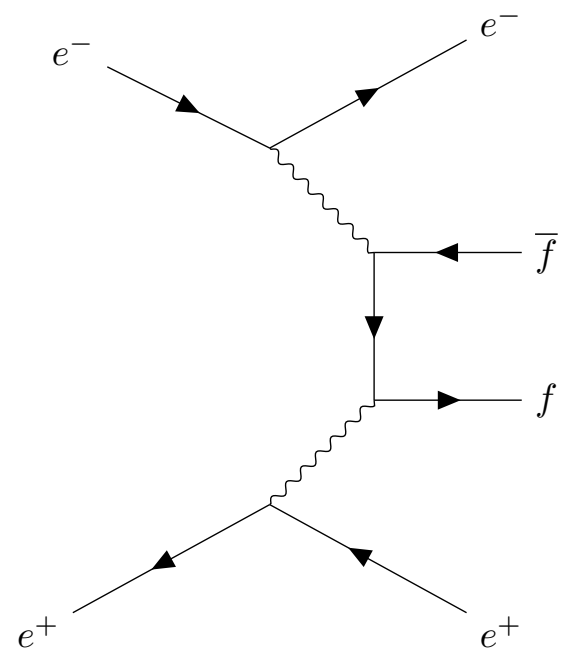

Figure 3.4.: Two photon process with 4 fermions in the final state. 


\section{SuperKEKB and Belle2}

\subsection{The Belle II experiment}

The Belle II detector is a general-purpose $4 \pi$ detector and the successor of the Belle experiment. Compared to Belle all subsystem were upgraded and improved. Only a few parts of Belle like the solenoid magnet and parts of the readout electronics were reused. The superconducting solenoid creates a magnetic field of $1.5 \mathrm{~T}$ to bend the tracks of electrically charged particles and measure their momentum. The design goal was to achieve at least the same performance as Belle while being able to handle the increased backgrounds due to the higher luminosity and nano beam scheme of SuperKEKB. In this section the various subsystems of Belle II will be presented, beginning at the most inner part (vertex detector) and going outwards from there. Figure 3.5 shows a CAD drawing of the Belle II detector. The following description is based on the Belle II technical design report [33].

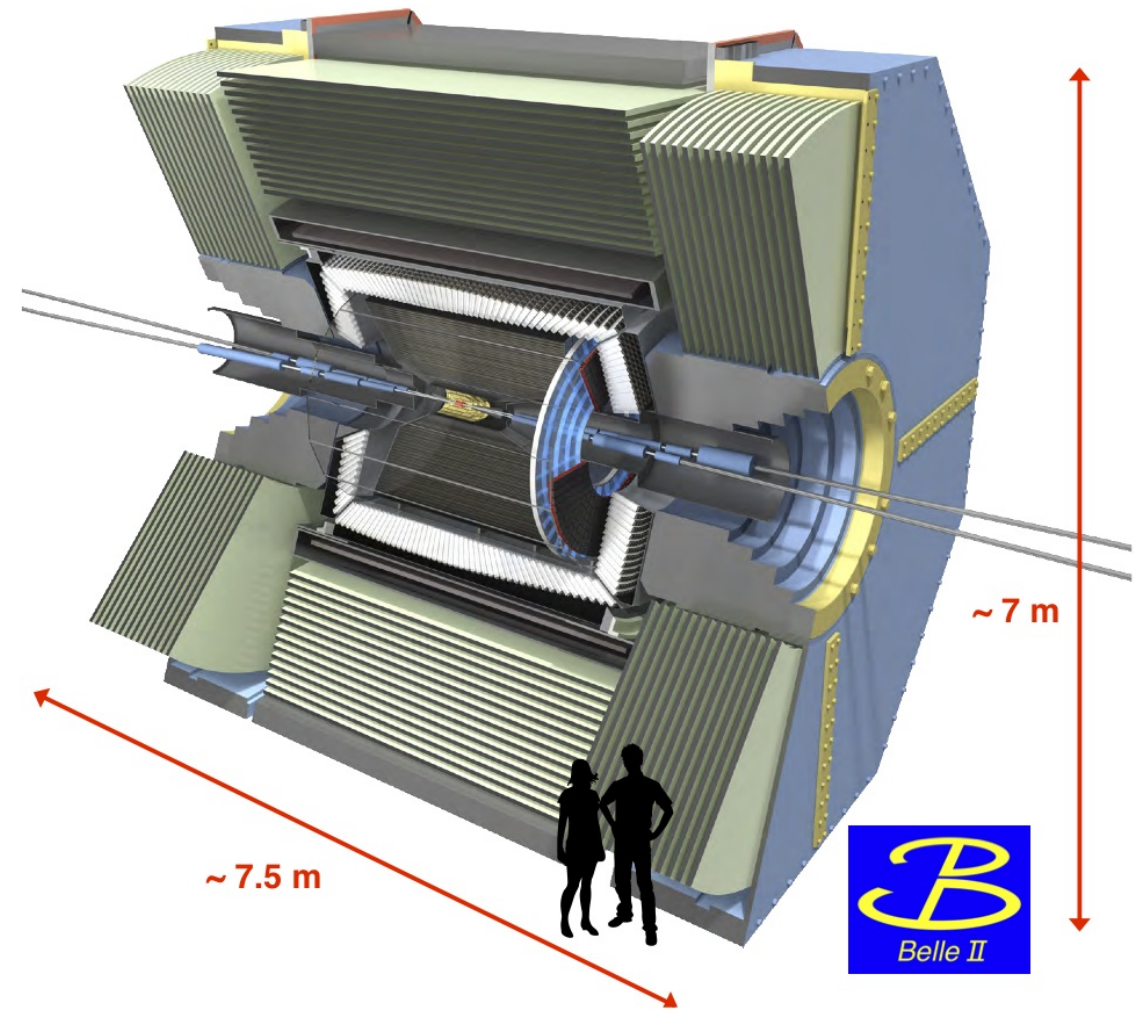

Figure 3.5.: CAD drawing of the Belle II detector. From [34]. 


\subsubsection{Vertex Detector (VXD)}

The vertex detector (VXD) is the part of Belle II which is closest to the interaction point (IP). The VXD consists of two layers of DEPFET pixel detector (PXD) ladders and four layers of double-sided silicon strip ladders forming the silicon vertex detector [35] (SVD). These six layers are positioned at radii of 14, 22, 38, 80, 115 and $140 \mathrm{~mm}$ around the beam pipe.

The PXD layers are organised in a windmill structure creating some overlap of the individual segments, which can be seen in fig. 3.6. In total the PXD consists of 40 halfladders, the smallest unit that can be operated on its own. For mechanical stability two half-ladders are glued together to form a (full) ladder. The VXD is used to reconstruct the decay vertices of the particles created in the electron positron interactions. Only for the two innermost layers pixels are used while the other four layers are strips. As pointed out before a high vertex resolution is required for time dependent measurements of $\mathrm{CP}$ violation. Other physical processes require a high resolution as well. As the focus of this thesis is the PXD, its components and its technology will be described in detail in chapter 4.

The SVD covers the angles between $17^{\circ}$ and $150^{\circ}$ and is built asymmetric because of the boost in $+z$ direction. The VXD is designed to be able to reconstruct low- $p_{t}$ tracks in the low $\mathrm{MeV}$ range.

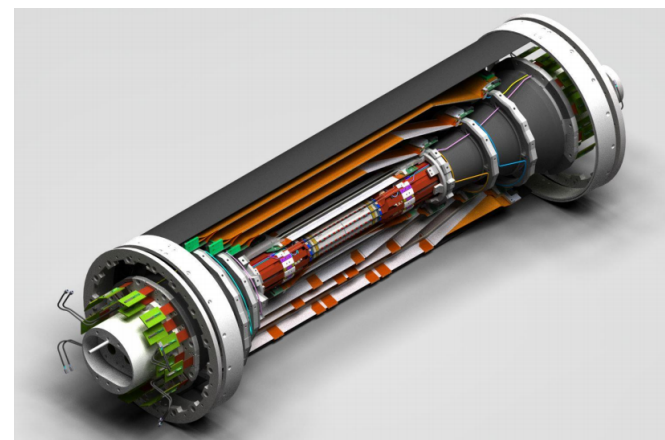

(a)

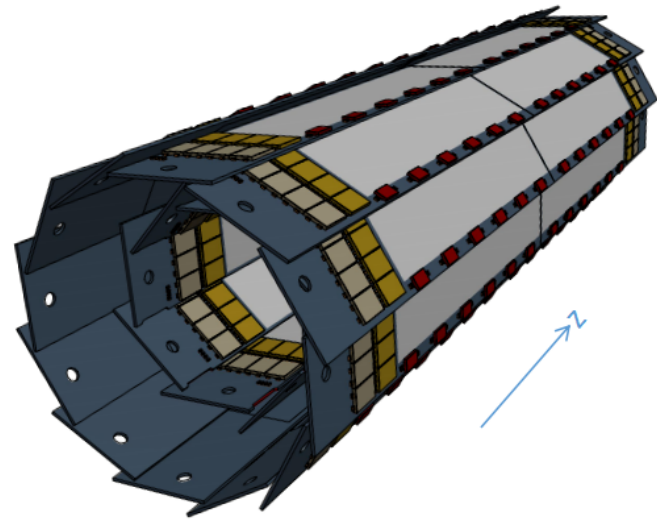

(b)

Figure 3.6.: CAD drawing of the full Belle II VXD system (a) and the PXD alone (b) showing the windwill arragement of the individual ladders. From [36].

\subsubsection{Central Drift Chamber (CDC)}

The central drift chamber (CDC) is used for tracking and momentum measurements of electrically charged particles. The chamber is filled with $\mathrm{He}-\mathrm{C}_{2} \mathrm{H}_{6}$ gas and 14336 sense wires with a thickness of $30 \mu \mathrm{m}$. A high positive voltage is applied to these wires. When 


\section{SuperKEKB and Belle2}

a particle flies through the chamber it ionises the gas, and the released electrons drift to the nearest wire, producing a current and measurable signal. As the VXD has a larger outer radius than in Belle, the CDC radii had to be adjusted as well. In Belle II the CDC starts at a radius of $160 \mathrm{~mm}$ and extends to $1130 \mathrm{~mm}$. The configuration of the wires was also modified as can be seen in fig. 3.7. In addition to the momentum measurement based on the curvature of tracks inside the $\mathrm{CDC}$, the chamber also delivers a three-dimensional trigger information for Belle II. The CDC can also be used for particle identification by measuring the energy loss within the gas volume, which is important for low energy particles that do not reach the particle identification systems outside of the CDC. To be able to cope with the increased background and occupancy, the readout electronics was also upgraded.

\section{Wire Configuration}
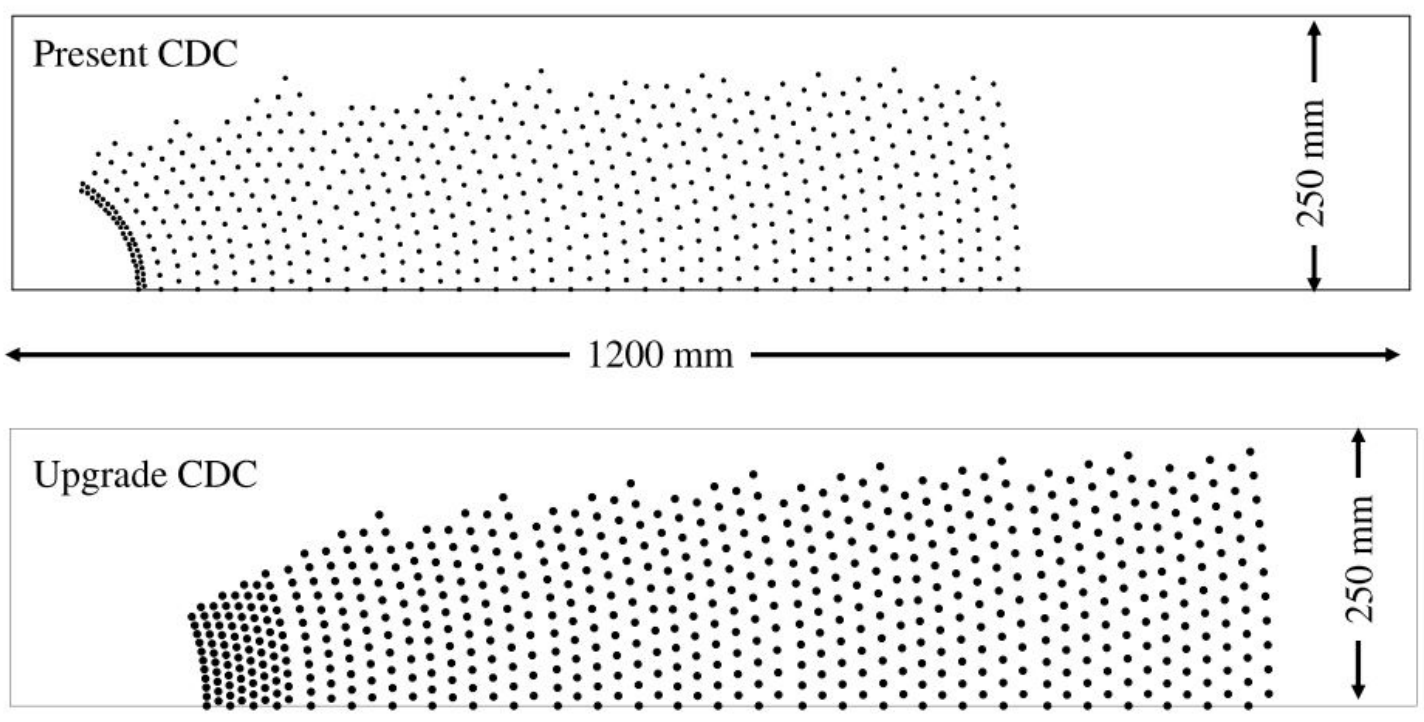

Figure 3.7.: Wire configuration of the old (left) and new (right) CDC. The number of layers was increased from 50 to 56 and the total number of wires has grown from 8400 to 14336. From [33].

\subsubsection{Particle Identification}

The next subsystem is the particle identification system (PID) which is used mainly to distinguish Kaons from pions. The design goal was to improve the separation power compared to Belle while at the same time decrease the material budget of the system. To achieve this goal the technology was switched from time-of-flight sensors (ToF) to timeof-propagation (TOP) counters. Both systems have in common that they use Cherenkov light to identify the particles. Cherenkov light is produced when a particle moves within a certain material faster than the phase speed of light. While Belle used areogel as the 
medium to generate Cherenkov light, the TOP system uses a quartz block. The angle $\theta_{c}$ under which the light is emitted depends on the velocity of particle. Together with the momentum measurement of the CDC this allows to identify the particle. Figure 3.8 visualises the working principle of a single TOP counter block. Part of the Cherenkov light is trapped inside the block because of total reflection and measured with photo detectors at the end of the block. Together with a precision timing measurement this creates a characteristic pattern in space and time.

For the endcaps of Belle II the aerogel approach is reused, the system is called proximityfocusing Aerogel Ring-Imaging Cherenkov detector (ARICH). The Cherenkov light produced in the aerogel is detected by a photon detector positioned a few centimetres behind where the photons form a "ring". The angle of the Cherenkov light determines the radii of these rings on the sensors. A photon detector with high granularity is required to measure the radii with high precision.

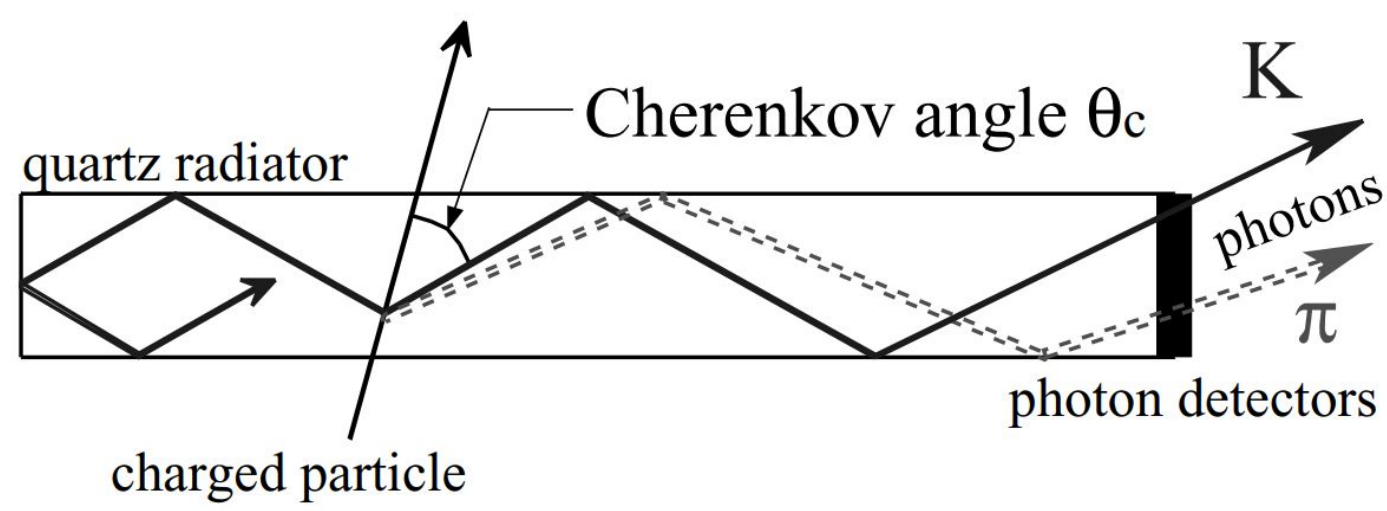

Figure 3.8.: Working principle of a Time-Of-Propagation (TOP) counter. From [33].

\subsubsection{Electromagnetic Calorimeter (ECL)}

The electromagnetic calorimeter (ECL) of Belle II uses CsI(Tl) scintillation crystals for the barrel part to measure the energy of particles crossing it. The barrel part has an inner radius of $1.25 \mathrm{~m}$ and is $3 \mathrm{~m}$ long. Mounted to the rear surface of the crystals are photodiodes, which detect the scintillation light produced within the crystals. Each crystal has a radiation length of $16.1 X_{0}$. When an electron or photon interacts with the crystals, an electromagnetic shower is created as the particle loses its energy. Apart from measuring the energy, the ECL is also used for particle identification (electrons). For the endcaps CsI crystals are used as they have a faster scintillation decay time which is needed because of the higher occupancy in the endcaps. The ECL also contributes trigger information to the global triggering system and is used to measure the luminosity. 


\section{SuperKEKB and Belle2}

\subsection{5. $K_{L}$ and muon detector (KLM)}

The most outward positioned subsystem is the $K_{L}$ and muon detector (KLM). Like the Particle Identification system it is subdivided in a barrel part and an endcap part. The barrel part is constructed in a sandwich design with $4.7 \mathrm{~cm}$ thick iron plates, which are also part of the solenoid system, and glass-electrode resistive plate chambers (RPC) to detect the particles. Muons show typically only a small or no signal in the ECL, but are detected in the KLM due to the thick iron plates providing 3.9 interaction lengths. The RPCs were also used in the Belle KLM system and proved to be a reliable system. They do, however, have a significant dead time which degrades their performance with increasing occupancy. For the barrel part, the increased background from SuperKEKB is expected to have only a small effect. The endcaps on the other hand are more susceptible to the background as the KLM system is not shielded as good as the barrel part. That is why scintillators are used for the endcaps instead of RPCs. The detection efficiency for muons with a momentum above $1 \mathrm{GeV}$ is $89 \%$ while the hadron fake rate is $1.3 \%$. 


\section{CHAPTER 4}

\section{Pixel Vertex Detector for Belle II}

As the most inner part of Belle II, the pixel vertex detector (PXD) is used to measure decay vertices with a very high precision. In total the PXD consists of nearly 8 million pixels, spread over a surface of $273.92 \mathrm{~cm}^{2}$. Because of its closeness to the interaction point and the beam, the PXD also suffers the most from radiation damage. This chapter will describe the working principle and the properties of the PXD starting with a short general introduction to semiconductor detectors, followed by an in depth description of the DEPFET technology and closing with the front- and back-end electronics needed to operate the PXD.

\subsection{Semiconductors}

Using Semiconductors is an efficient way to detect most of the particles that are created in high energy physics. Semiconductors are materials with an electrical conductivity below conductors but above isolators. In the band model they have a small gap between their valence and their conduction band. A prominent example of a semiconductor is Silicon $(\mathrm{Si})$ with a band gap of $1.1 \mathrm{eV}$. The process of doping describes the creation of impurities within the semiconductor crystal. For Si, which has 4 valence electrons, this can be done with Phosphor, which has 5 valence electrons. The additional electron cannot form a covalent binding and can move freely within the crystal. This is called $n$-doping. Using Aluminium, which has 3 valence electrons, the same effect with an additional positive charge ("hole") can be created, which is then called $p$-doping. To use the semiconductor as a detector, a p-n junction is built. At this junction the electrons from the $\mathrm{n}$-doped side diffuse to the $\mathrm{p}$-doped side and the holes from the $\mathrm{p}$-doped side diffuse to the $n$-doped side. The diffusion is stopped because of the electric field that is created by this movement of charge carriers. Around the junction a depletion region is created with no free charge carriers. By applying an external voltage it is possible to 


\section{Pixel Vertex Detector for Belle II}

increase or decrease the size of this region. For a particle detector a large depletion area is desired. When a particle (e.g. an electron) traverses the depletion area of the detector, it interacts with the electrons of the crystal which brings them from the valence band to the conductor band, creating new electron-hole pairs. For Si the energy required to create an electron-hole pair is $3.6 \mathrm{eV}$. These pairs are created along the particles trajectory within the detector and they begin to drift as they feel the force of the electric field. This creates a current which can be measured. A sketch of this process can be seen in fig. 4.1 .

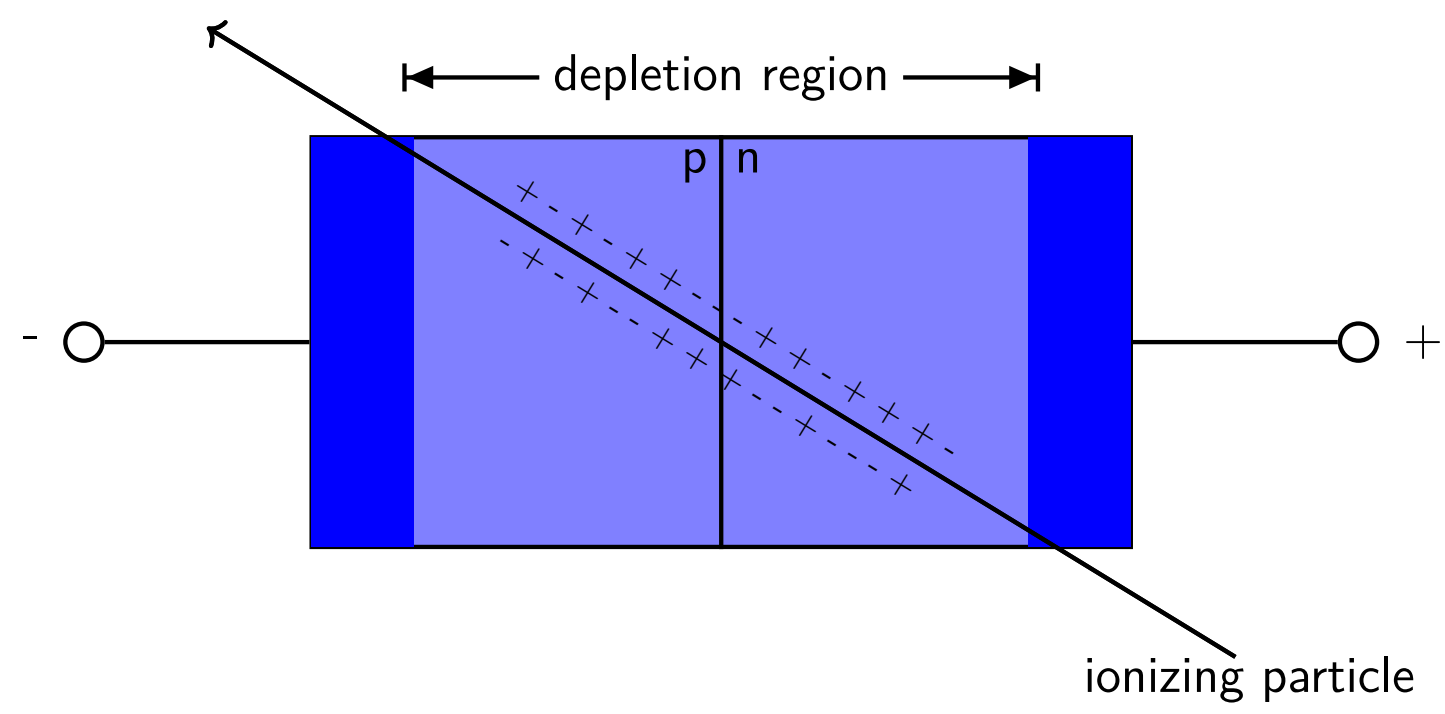

Figure 4.1.: Basic principle of a semiconductor detector. A silicon strip is doped to form a diode with a p-n junction. The diode is reverse biased to create a depletion region with no free charge carriers. When an ionising particle crosses the volume of the diode, electron-hole pairs are created which drift to the external contacts resulting in an electrical current.

The concept can be expanded to a strip detector by adding multiple electrodes on top of the depleted volume, see fig. 4.2. The charge carriers will drift to the nearest electrode, which gives information about the position of the crossing particle. In a double sided design these strips are on both sides of the volume rotated by 90 degrees relative to each other, giving a two dimensional reading. If $d_{x / y}$ is the distance between the strips in $x$ and $y$ direction and the readout is purely binary (hit or no hit), the resolution $\sigma_{x / y}$ of the detector is given as:

$$
\sigma_{x / y}=\frac{d_{x / y}}{\sqrt{12}}
$$

In case of the SVD of Belle II, which has strip pitches between $50 \mu \mathrm{m}$ and $240 \mu \mathrm{m}$, this means the resolution is between $14 \mu \mathrm{m}$ and $70 \mu \mathrm{m}$. 


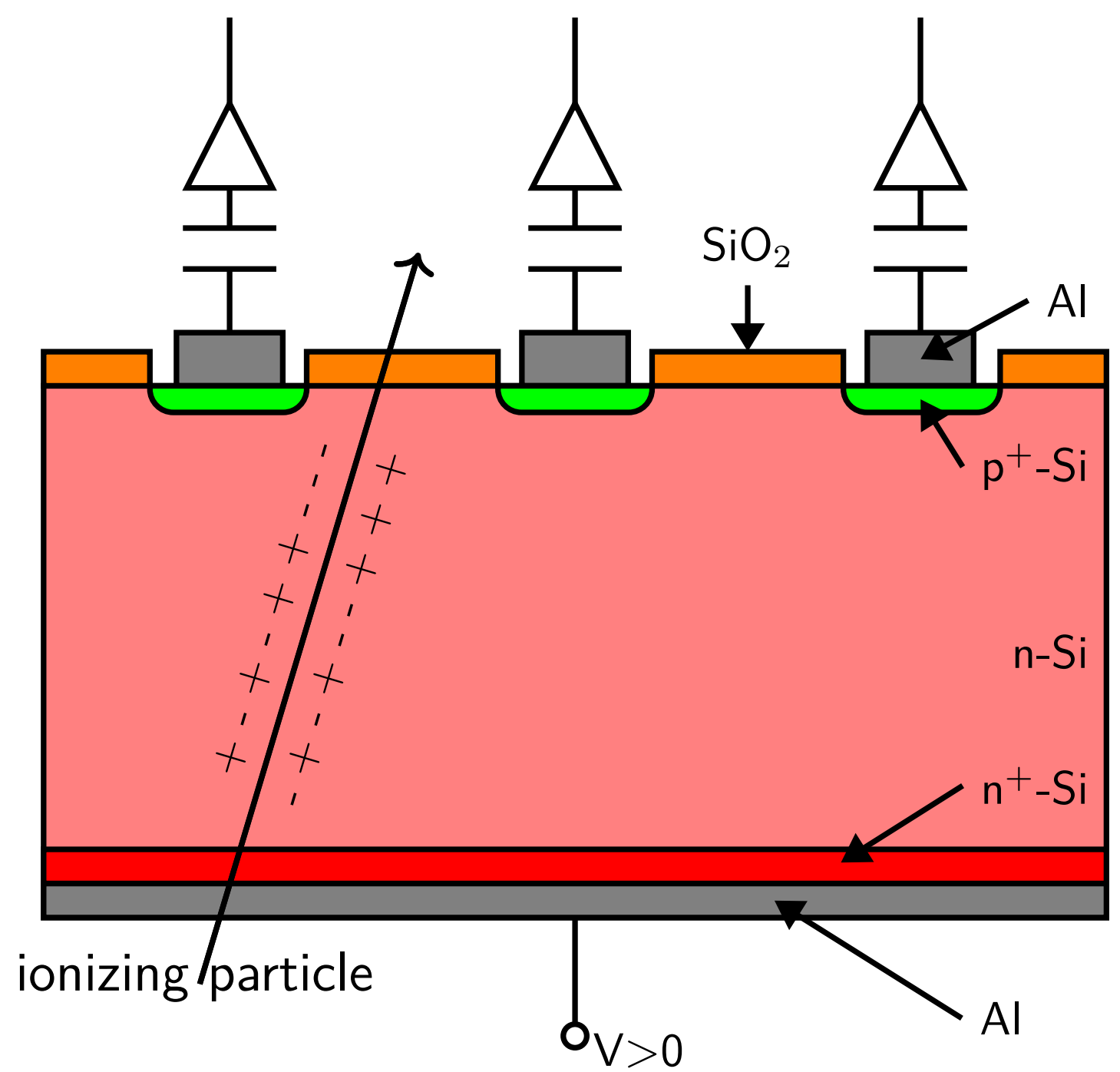

Figure 4.2: Simplified sketch of a strip detector. Depletion of the detector volume is achieved by a voltage applied at the backside of the detector. The created electron-hole pairs drift to the nearest electrode at the top side, giving information about where the particle traversed the detector. 


\section{Pixel Vertex Detector for Belle II}

\subsection{MOSFET}

Before going into detail about the DEPFET technology, it is necessary to understand the metal-oxide-semiconductor field-effect transistor (MOSFET). The following decription is based on [37]. MOSFETs are a special kind of field-effect transistors (FETs). As all FETs they are steered by voltages instead of currents like bi-polar transistors. Each MOSFET has three contacts: Source, Gate and Drain. The gate contact is used to steer the current that flows between source and drain. In general, there are two types of MOSFETs: n-channel and p-channel ones. The type determines whether electrons or holes are used as charge carriers for the source-drain connection. Because of the higher mobility of electrons, the n-channel design is more frequently used. However, since the DEPFET uses a p-channel, the p-channel MOSFET will be explained here. The structure of a p-channel MOSFET can be seen in fig. 4.3. The main part of the

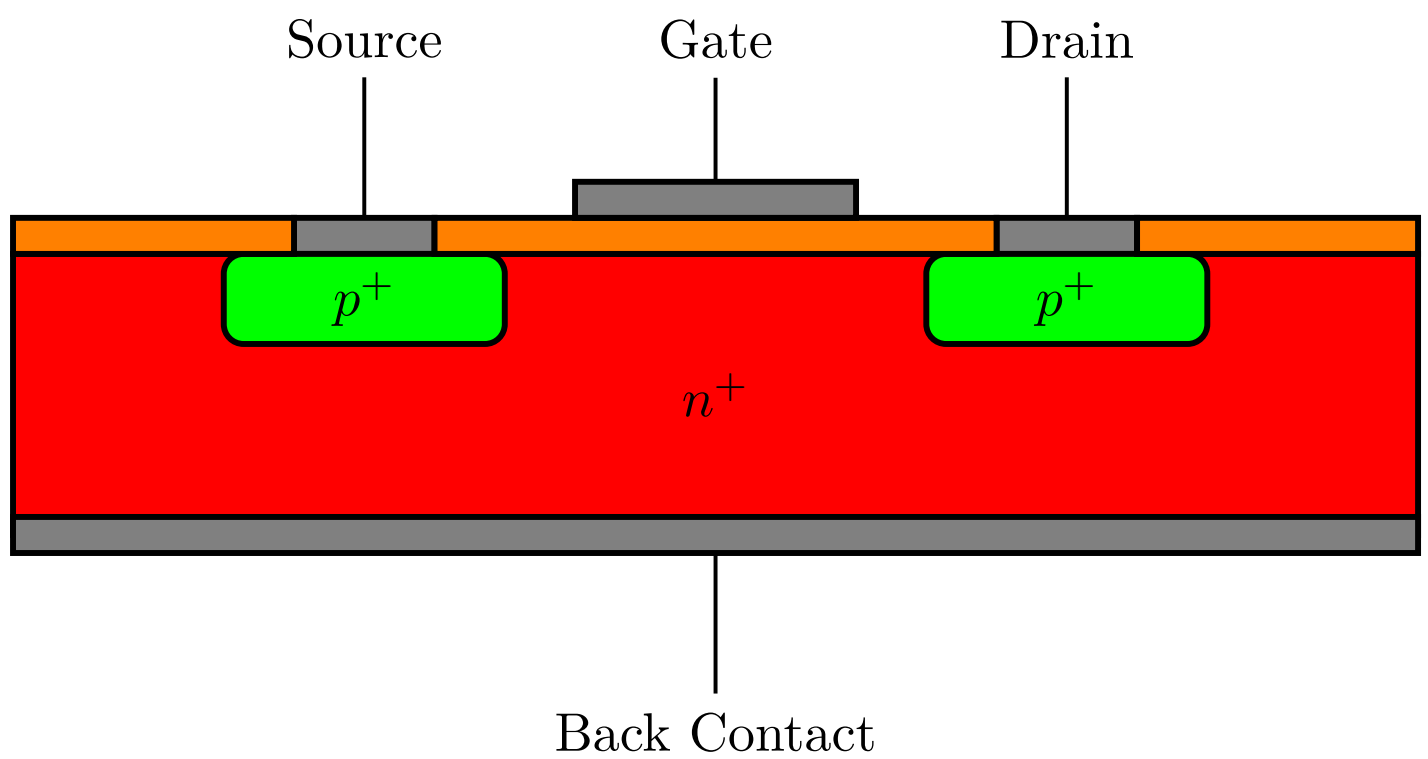

Figure 4.3.: Schematic drawing of a p-channel MOSFET. The substrate (red) is n-doped while the source and drain regions (green) are p-doped. The metal gate contact (black) is isolated from the substrate by a thin oxide layer $\left(\mathrm{SiO}_{2}\right)$. When no voltages are applied no current can flow from source to drain because of the pnp junction.

MOSFET is n-doped silicon (substrate). Within this substrate are two p-doped regions, forming the source and drain part. Because of this arrangement a pnp junction (two pn junctions back to back) is formed, which blocks any current from flowing. The gate metal contact is located between source and drain and separated from the substrate by a thin isolator (e.g. $\mathrm{SiO}_{2}$ ). The back contact can be steered individually but is often shortened with the source, leaving only source, drain and gate as free contacts. Assuming source and drain are at ground level, a negative voltage applied to the gate contact has 
the following effect: The electrons below the gate (in the n-doped region) are pushed further into the substrate, creating a depletion area just below the gate. This effect is enhanced by the p-doped source and drain regions, from which free positive charge carriers (holes) drift towards the gate because of the negative voltage. This creates a $\mathrm{p}$-channel, connecting drain and source. Because this $\mathrm{p}$-channel is formed within the $\mathrm{n}$ doped substrate, it is also called inversion channel. Once the voltage applied at the gate is below the threshold voltage $V_{\mathrm{thr}}$, a current starts to flow between source and drain. The value of $V_{\text {thr }}$ depends on the doping concentrations and the width/length of the transistor. Decreasing the gate voltage further increases the number of charge carriers in the channel and therefore increases the current.

To have any current $I_{D}$ flowing from drain to source, the voltage between source and drain $V_{D S}$ must of course be greater than zero. The magnitude of this voltage also determines whether the transistor is operated in the so-called linear mode or saturation mode. In the linear mode increasing $V_{D S}$ leads to an almost linear increase of $I_{D}$. This behaviour changes though at a certain point. Assuming the source contact is connected to the ground and $V_{G}$ is constant, $V_{D S}$ can be seen as a voltage drop across the transistor. This does have an influence on the gate voltage as it also decreases from $V_{G}$ at the source contact to $V_{G^{-}} V_{D S}$ at the drain contact. On other hand, this decreases the depth of the p-channel, deforming it more and more. Once $V_{D S}$ is above the saturation point $V_{D S, s a t}$, the channel is completely pinched off and a further increase of $V_{D S}$ has no additional effect on the current $I_{D}$. Figure 4.4 shows a visualisation of this process.

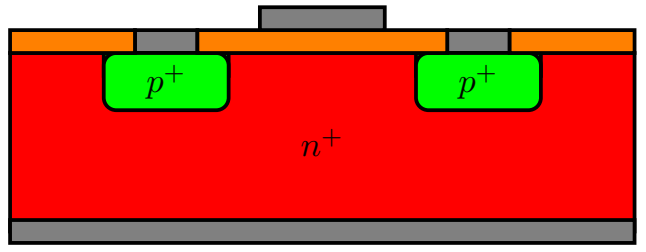

(a)

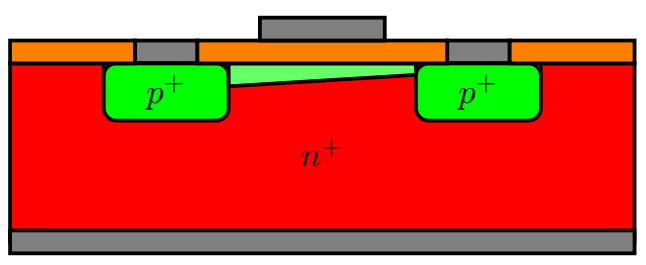

(c)

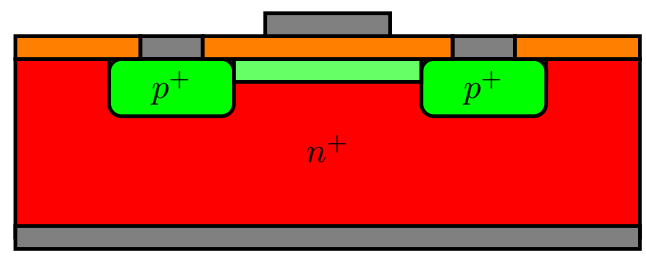

(b)

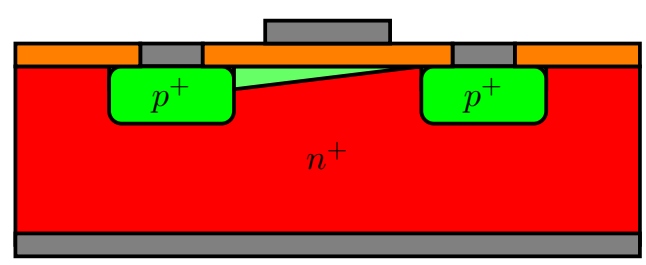

(d)

Figure 4.4.: Formation of the induced p-channel. Below the threshold voltage $V_{\text {thr }}$ there is no channel between source and drain (a). If $V_{G}$ is above $V_{\text {thr }}$ the channel is formed and a current can flow (b). With increasing $V_{D S}$ the channel is deformed (c). Above the saturation voltage $V_{\text {sat }}$ the channel is fully pinched off and the transistor operates in the saturation regime $(\mathrm{d})$.

The drain current $I_{D}$ in the linear region before $V_{D S}$ reaches the saturation value can 


\section{Pixel Vertex Detector for Belle II}

be described by

$$
I_{D}=\mu_{p} C_{o x} \frac{W}{L}\left(\left(V_{G}-V_{\mathrm{thr}}\right) V_{D S}-\frac{V_{D S}^{2}}{2}\right),
$$

where $W$ is the width of the gate, $L$ the length of the gate, $\mu_{p}$ the mobility of the (positive) charge carriers and $C_{o x}$ the capacitance per unit gate area, determined by its oxide thickness since the gate can be seen as a plate capacitor. In the saturation region the drain current is independent of $V_{D S}$ and given as

$$
I_{D}=\frac{1}{2} \mu_{p} C_{o x} \frac{W}{L}\left(V_{G}-V_{\mathrm{thr}}\right)^{2}
$$

Here only $V_{G}$ steers the drain current and the amplification $g_{m}$ of the current is defined as

$$
g_{m}=\frac{\partial I_{D}}{\partial V_{G}}=\mu_{p} C_{o x} \frac{W}{L}\left(V_{G}-V_{\mathrm{thr}}\right)
$$

Figure 4.5 shows the behaviour of the drain current for different values of $V_{G}$ in the linear and saturation regime based on eqs. (4.2) and (4.3). However, it has to be noted that these equations are based on a simplified model. In reality, $V_{D S}$ still has an effect even in the saturation region as it reduces the length of the conductive channel slightly. 


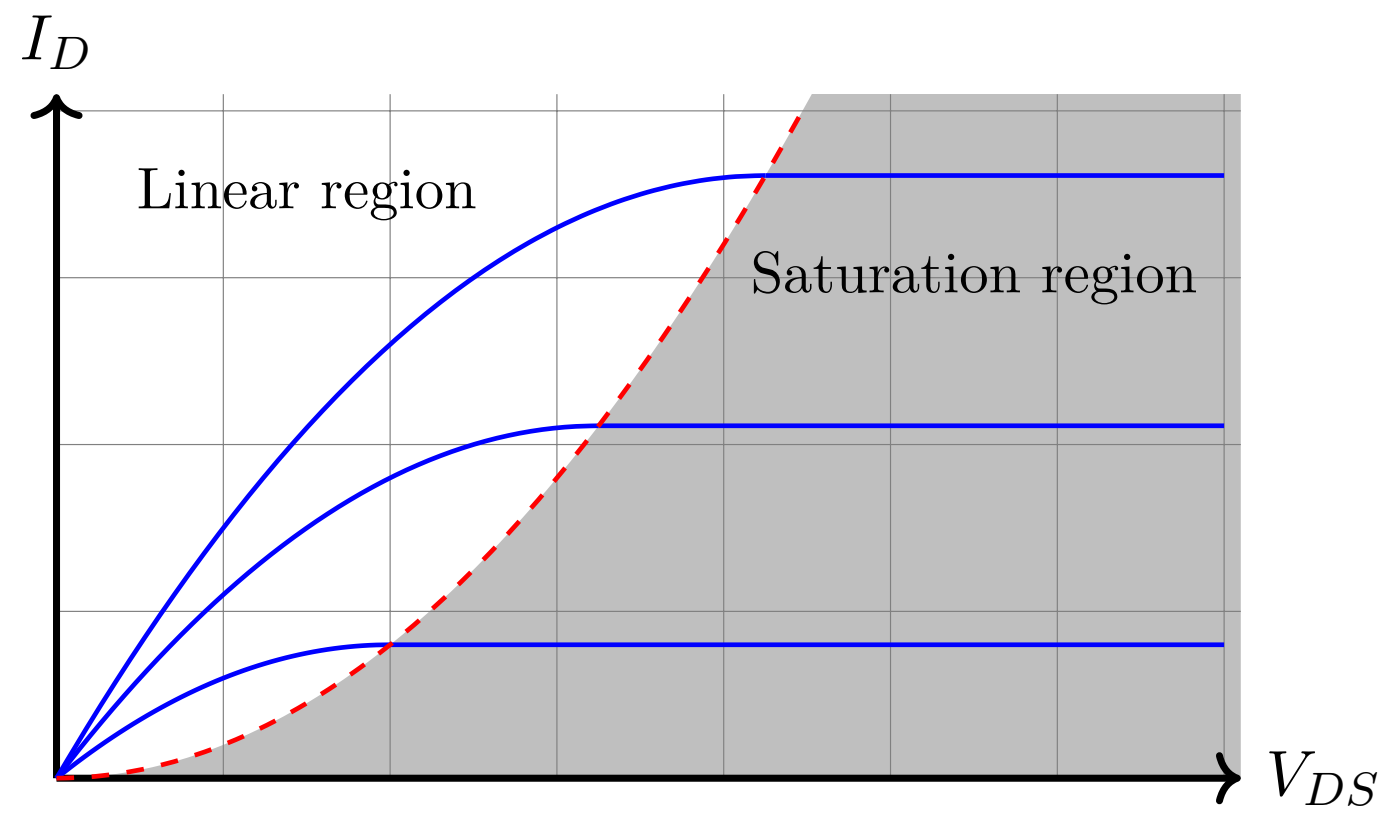

Figure 4.5.: Drain current $I_{D}$ as a function of drain source voltage $V_{D S}$ for different values of $V_{G}$. The red dashed line separates the linear region (left) from the saturation region (right). 


\section{Pixel Vertex Detector for Belle II}

\subsection{DEPFET}

With a basic knowledge of the MOSFET it is possible to extend the concept to the DEPFET technology. This section covers the general working principle of a DEPFET but also describes the specific implementation used for the PXD in Belle II. The depleted p-channel field-effect transistor (DEPFET) technology was first described in 1987 by J. Kemmer and G. Lutz [38]. The PXD for Belle II marks the first time that this technology is used within a large collider experiment. It follows the principle of the MOSFET but adds an additional "internal" gate and a second FET for the so-called clear mechanism. Figure 4.6 shows a sketch of a simple DEPFET cell. Like the MOSFET, the DEPFETs

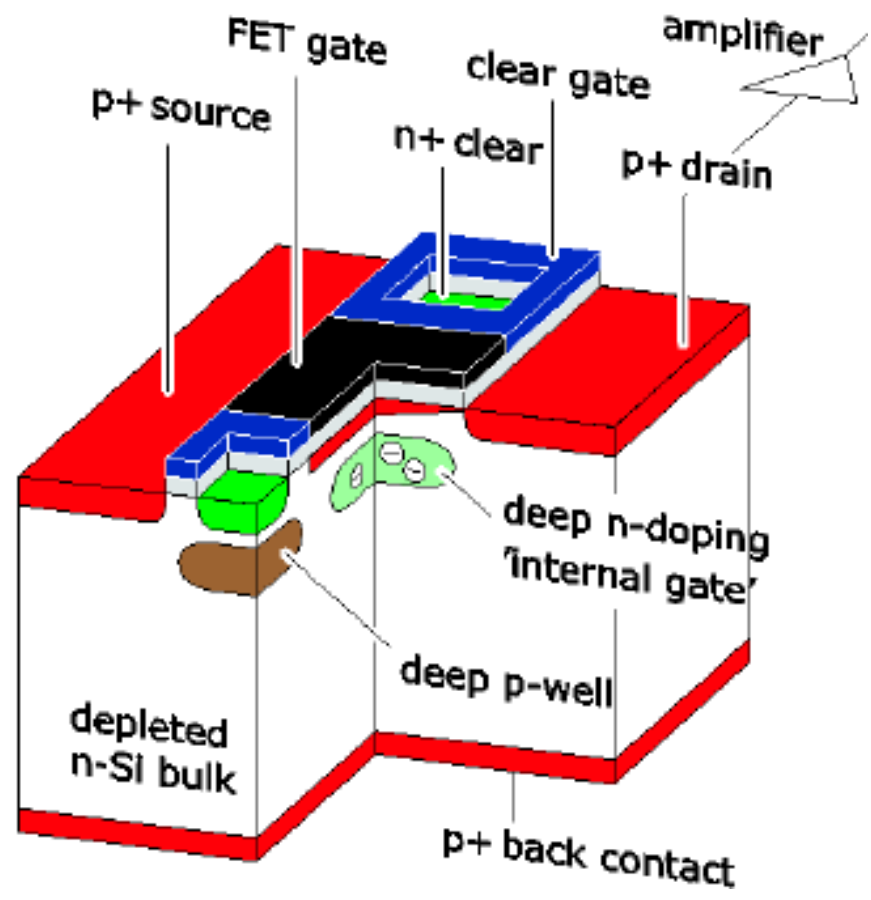

Figure 4.6.: Sketch of a DEPFET cell. From [39].

main volume is a n-doped Silicon substrate, in the following called bulk. On top of the bulk are the source, drain and gate contacts forming the MOSFET. This FET is operated in the saturation regime. As the DEPFET design has more than one gate, this gate will be called the FET gate from here on.

The first major difference compared to the simple MOSFET is the additional n-doped area below the FET gate, called the internal gate. To function as a detector, on the backside of the bulk a large negative voltage is applied to deplete the whole volume. When an ionising particle crosses the depleted bulk and creates electron-hole pairs, the electrons drift to the internal gate (because the doping is stronger than in the rest of the bulk) and are collected there, while the holes drift to the backside and are removed from the volume. The negatively charged electrons inside the internal gate now have the 
same effect as the negative voltage applied at the FET gate, just from "the other side". This second gate strengthens the p-channel which increases the drain current $I_{D}$. This is the main idea behind the DEPFET detector. It collects electrons and thereby charge $q_{i}$ inside its internal gate, which effectively adds another voltage term $V_{i}$ in eq. (4.3), giving

$$
\begin{aligned}
I_{D} & =\frac{1}{2} \mu_{p} C_{o x} \frac{W}{L}\left(V_{G}+V_{i}-V_{\mathrm{thr}}\right)^{2} \\
& =\frac{1}{2} \mu_{p} C_{o x} \frac{W}{L}\left(V_{G}+\frac{q_{i}}{C_{o x} W L}-V_{\mathrm{thr}}\right)^{2} .
\end{aligned}
$$

This internal amplification $g_{q}$ of the drain current can then be derived as

$$
\begin{aligned}
g_{q} & =\frac{\partial I_{D}}{\partial q_{i}} \\
& \left.=\frac{\mu_{p}}{L^{2}}\left(V_{G}+\frac{q_{i}}{C_{o x} W L}-V_{\mathrm{thr}}\right)\right) V_{i}=\frac{q_{i}}{C_{o x} W L} \ll V_{G} \\
& \approx \frac{\mu_{p}}{L^{2}}\left(V_{G}-V_{\mathrm{thr}}\right) \\
& =\frac{g_{m}}{C_{o x} W L} .
\end{aligned}
$$

Here the fact was used that the voltage induced by the charge in the internal gate is much smaller than the external gate voltage. As eq. (4.7) shows $g_{q}$ depends only on the working point $\left(V_{G}-V_{\mathrm{thr}}\right)$ of the DEPFET. Therefore, the drain current increases linearly with the deposited charge in the internal gate. As a consequence measuring the (increased) drain current and comparing it to the original one (with an empty internal gate) allows to directly measure the charge in the internal gate. The significant advantage here is that the signal of the particle is amplified internally compared to other detector designs where an external amplification is required.

After each mesurement/readout the internal gate has to be cleared of any charge to be ready for the next readout. To achieve this a second transistor is built into the DEPFET cell. This transistor is directly connected to the internal gate, which serves as the source of the clear transistor. The clear gate is located on top of the bulk next to the FET gate. The drain of the transistor is simply called clear contact and is a n-doped region below the surface. Figure 4.7 shows a sketch of the DEPFET as a simplified electric circuit. It highlights how the transistors are connected to each other. When the clear process is started, the clear transistor is activated and electrons from the internal gate are pulled to the $\mathrm{n}$-doped clear contact. There are, however, several complications with this approach. During the charge collection phase electrons could drift to the clear contact instead of the internal gate. To prevent this a p-doped well is placed below the clear contact. Due to technical constraints the clear transistor is not steered directly by the gate but by the voltage applied to the clear contact, which is capacitively coupled 


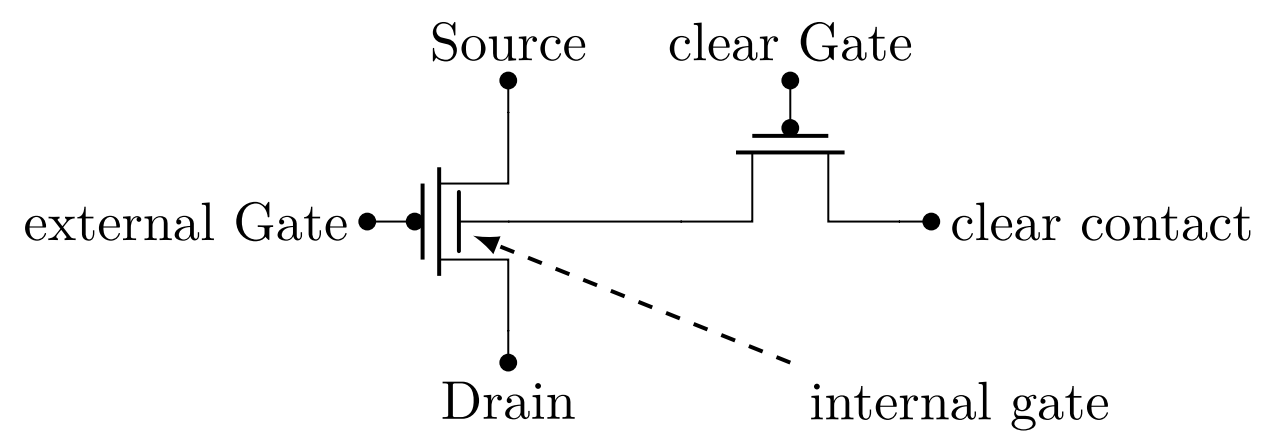

Figure 4.7.: Simplified representation of the DEPFET as an electric circuit.

to the clear gate ${ }^{1}$. During the clear process a large positive voltage is applied, forming a n-channel between the internal gate and the clear gate through which the electrons flow. After the process is completed, the voltage is lowered and the channel is closed. As the internal gate is now empty, the cell is back to the initial state and ready to collect new charges.

\subsection{Radiation Damage Effects on the DEPFETs}

Understanding the PXD also means to understand how ionising radiation affects the sensor, especially the DEPFETs. In general, radiation effects on silicon detectors and FETs are categorised into two types: surface damage and bulk damage. In the following a short overview of these two types will be given.

\subsubsection{Bulk Damage}

Whenever heavy particles like neutrons or protons cross the bulk of the sensor, they interact with the atoms of the silicon volume. In these interactions the atoms are kicked out of their position (primary knock-on atoms or PKAs) in the lattice which leads to so-called crystal defects. The PKAs travel through the lattice while they lose their energy and create further defects, which leads to the formation of crystal defect clusters. The consequences of these kind of damages are an increased leakage current, reduced collection efficiency due to additional trapping centres, and eventually type inversion (converting $\mathrm{n}$-doped silicon into p-doped). A more detailed description of the damage mechanisms is given in [40].

The amount of damage done by a traversing particle does not only depend on its energy, but also on the type of the particle. Using the NIEL (Non-Ionising Energy Loss) scaling hypothesis, it is possible to compare the damage of different particles and energies. The

\footnotetext{
${ }^{1}$ Steering the clear gate directly would require additional lines, and the space available on a PXD
} half-ladder is limited. 
idea is that any particle fluence $\Phi$ can be reduced to an equivalent $1 \mathrm{MeV}$ neutron fluence $\Phi_{\text {eq }}$ that causes the same damage:

$$
\Phi_{\mathrm{eq}}=\alpha \cdot \Phi
$$

The conversion factor $\alpha$ can be acquired by comparing the displacement damage crosssection of neutrons and the particle type that is to be compared. If the particle has a fixed energy $E_{0}$, the conversion factor is given as

$$
\alpha=\frac{D\left(E_{0}\right)}{D\left(E_{\text {neutron }}=1 \mathrm{MeV}\right)} .
$$

At Belle II the main source of particles affecting the PXD are $\approx 6 \mathrm{MeV}$ electrons. Simulations have shown that the expected NIEL damage to the PXD after 10 years of Belle II operation is $\approx 10^{13} 1 \mathrm{MeV} \mathrm{n}$ eq $/ \mathrm{cm}^{2}$. Previous studies [41] have shown that the noise increase at this level of bulk damage is manageable and the type inversion only becomes an issue at $\approx 10^{14} 1 \mathrm{MeV} \mathrm{n}$ eq $/ \mathrm{cm}^{2}$. Bulk damage is therefore not considered an issue for the operation of the PXD.

\subsubsection{Oxide Damage}

The second type of damage is surface or oxide damage. It describes damage done to the gate oxide layers between the metal contacts and the silicon volume. As described before a DEPFET cell has two gates, the main DEPFET gate and the common clear gate (ccg). Both gates are affected by ionising radiation and experience damage. When an ionising particle traverses the gate oxide layer $\left(\mathrm{SiO}_{2}\right)$, it interacts with the material and electron-hole pairs are created. The physical quantity to describe the amount of created pairs is the absorbed energy, called total ionising dose or just dose. The absorbed dose $D$ of any material is defined as the absorbed energy $E$ per mass $m$ and is measured in Gy:

$$
\begin{aligned}
D & =\frac{E}{m} \\
1 \mathrm{~Gy} & =1 \frac{\mathrm{J}}{\mathrm{kg}}
\end{aligned}
$$

The energy required to generate one electron-hole pair in $\mathrm{SiO}_{2}$ is $\approx 18 \mathrm{eV}$ [42]. Using the density of $\mathrm{SiO}_{2}\left(2650 \frac{\mathrm{kg}}{\mathrm{m}^{3}}\right)$ this gives an initial electron-hole pair density per absorbed dose of $\approx 9.19 \cdot 10^{20} \frac{\text { pairs }}{\mathrm{m}^{3} \mathrm{~Gy}}$. Due to initial recombination processes this number is quickly decreased though. This recombination is influenced by the electric field in the oxide and the line density of charge pairs. While there is no analytical solution for an arbitrary line density, there are solutions for special cases [43] and experimental data available [44]. While the electrons drift outside of the volume rather quickly (if the FET is biased), the holes move much slower and remain in the oxide volume for a longer time [45]. In general, the movement of the holes towards the $\mathrm{SiO}_{2} / \mathrm{Si}$ border can be described as a continuous-time-random-walk (CTRW) [46]. The temperature as well as the electric 


\section{Pixel Vertex Detector for Belle II}

field influence their movement significantly. The specific transport mechanism is referred to as polaron hopping [47] where the hole moves through the lattice together with the created lattice distortion. Due to their (slow) movement, the concentration of holes is highest near the $\mathrm{SiO}_{2} / \mathrm{Si}$ border [48] where they are trapped. The holes are trapped there because the oxidation in this region of the Si is not complete. Due to a missing oxygen atom, a weak $\mathrm{Si}-\mathrm{Si}$ bond is created. The hole breaks this bond and relaxes the

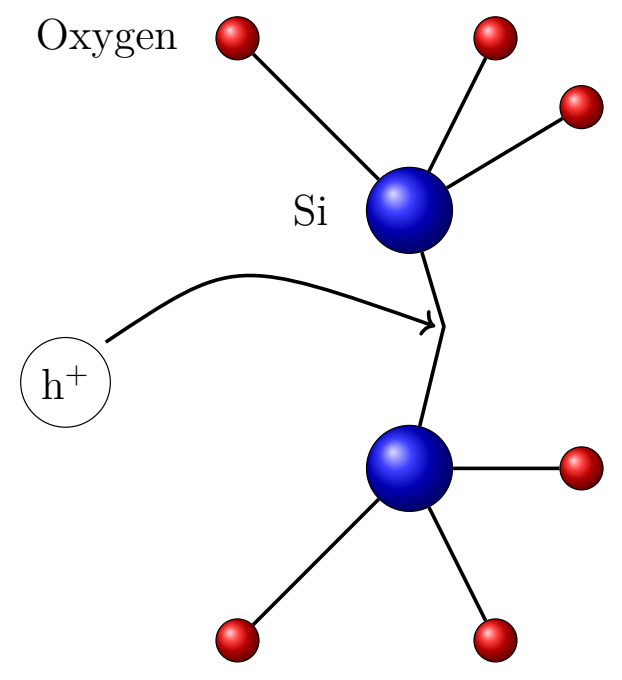

Neutral oxygen vacancy defect

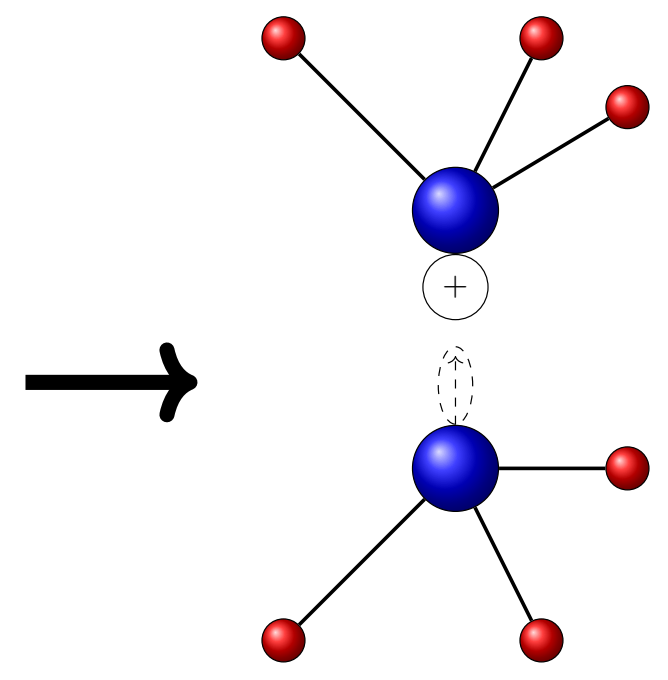

Positively charged $E^{\prime}$ centre

Figure 4.8.: Hole trapping mechanism.

lattice in an asymmetric way, creating a $E^{\prime}$ centre structure with positive charge [49]. A sketch of this process is shown in fig. 4.8.

In addition to the trapped holes, there is a second effect due to the radiation, the creation of electronic states within the $\mathrm{Si}$ bandgap. They are located near the $\mathrm{SiO}_{2} / \mathrm{Si}$ border and called interface traps. In the past, various models have been discussed to explain the formation of these traps. The model that is widely accepted nowadays describes the creation of these traps by a two-stage $\mathrm{H}^{+}$process $[50,51]$. While the holes move through the lattice, they release bound hydrogen atoms (protons) that drift towards the $\mathrm{SiO}_{2} / \mathrm{Si}$ interface. There the protons react with hydrogen-passivated defects and form $\mathrm{H}_{2}$ molecules. These molecules diffuse out and leave a charge defect behind.

Both effects have in common that they create charges. These charges affect directly the threshold voltage $V_{\text {thr }}$ of the FET through electrostatic effects.

$$
\Delta V_{\mathrm{thr}}=-\frac{1}{\epsilon_{\mathrm{ox}}} \int_{0}^{d_{\mathrm{ox}}} x \rho_{\mathrm{ox}}(x) \mathrm{d} x
$$


Here $\epsilon_{\mathrm{ox}}$ is the dielectric constant of the oxide, $d_{\mathrm{ox}}$ is the oxide thickness, $\rho_{\mathrm{ox}}$ is the volume density of charge in the oxide and $x$ is the position in the oxide. This expression is often simplified by introducing an equivalent charge located at the border region instead of describing the position of the holes in the oxide. The shift due to the positive charge of the holes $Q_{\mathrm{ox}}$ is then given as

$$
\Delta V_{\text {oxide }}=-\frac{Q_{\mathrm{ox}}}{C_{\mathrm{ox}}},
$$

where $C_{\mathrm{ox}}$ is the capacitance of the oxide. The charge due to the aforementioned interface traps can be positive or negative (or even neutral) depending on the position of the Fermi potential. For p-channel MOSFETs the Fermi level is above the midband energy and for n-channel MOSFETs below [52]. The contribution from the charge $Q_{\text {it }}$ to the threshold shift is analogue to the one from the holes in the oxide and given as

$$
\Delta V_{\text {interface }}=-\frac{Q_{\mathrm{it}}}{C_{\mathrm{ox}}} .
$$

The total shift of the threshold voltage is given as the sum of both components:

$$
\Delta V_{T}=\Delta V_{\text {oxide }}+\Delta V_{\text {interface }} \text {. }
$$

For p-channel MOSFET both contributions (holes and interface traps) are negative, i.e. shift the threshold voltage to more negative values. For n-channel MOSFETs the shift can be positive or negative, depending on the design of the FET.

\subsubsection{Oxide Damage Annealing}

Although the holes trapped in the oxide are rather stable, they can be removed by a process called annealing. Annealing processes are quite slow and can stretch over hours, days or years. They are highly dependend on the conditions in the oxide, especially temperature and electric fields. At room temperature tunnelling is the dominant mechanism responsible for annealing whereas at high temperatures, thermal excitation becomes more and more important. This is also the reason why irradiated samples are often heated up to speed up the annealing process. Characteristic for annealing processes is also that the annealing rate is highest directly after an irradiation and then decreases. Experiments [53] have shown that there is also an effect of temporary annealing where a sample seemed to be annealed fully but showed oxide damage effects again after several hours. This behaviour was explained in a model by Lelis et al. [54-56]. In this model electrons from the substrate tunnel to the neutral Si that was created through the hole trapping process (see fig. 4.8). By doing so a dipol structure is formed and the electron can tunnel back and forth to the substrate. This explains the observed temporary annealing. If the negative and positive $\mathrm{Si}$ atoms are located close enough to each other the coulomb force is strong enough to pull them together and reform the broken bound, which corresponds to the observed permanent annealing. 


\section{Pixel Vertex Detector for Belle II}

\subsection{PXD half-ladder and Front-end readout}

This section covers the smallest functional part of the PXD, the half-ladder. The halfladder contains a DEPFET matrix which is made up of $250 \times 768$ individual pixels. In addition there are 3 types of control chips. These ASICs (application-specific integrated circuits) are needed to control the rolling shutter readout, digitise the drain currents and process the data.

Figure 4.9 shows a drawing of a half-ladder including the aforementioned ASICs. The ASICs at the long side of the matrix are called switchers [57]. They switch the FET gate voltage and the clear contact voltage between their on and off values. At the bottom side of the matrix where the drainlines of the pixels end, 4 pairs of the drain current digitizers [57] (DCD) and the data handling processors [58] (DHPs) are located. The DCDs primary function is the digitisation of the drain currents, while the DHP processes the digitised information before it is sent out to the back-end readout electronics. The connection to the back-end electronics is realised by a Kapton flex cable, through which the power and data lines are routed. Each DCD-DHP pair is responsible for the readout

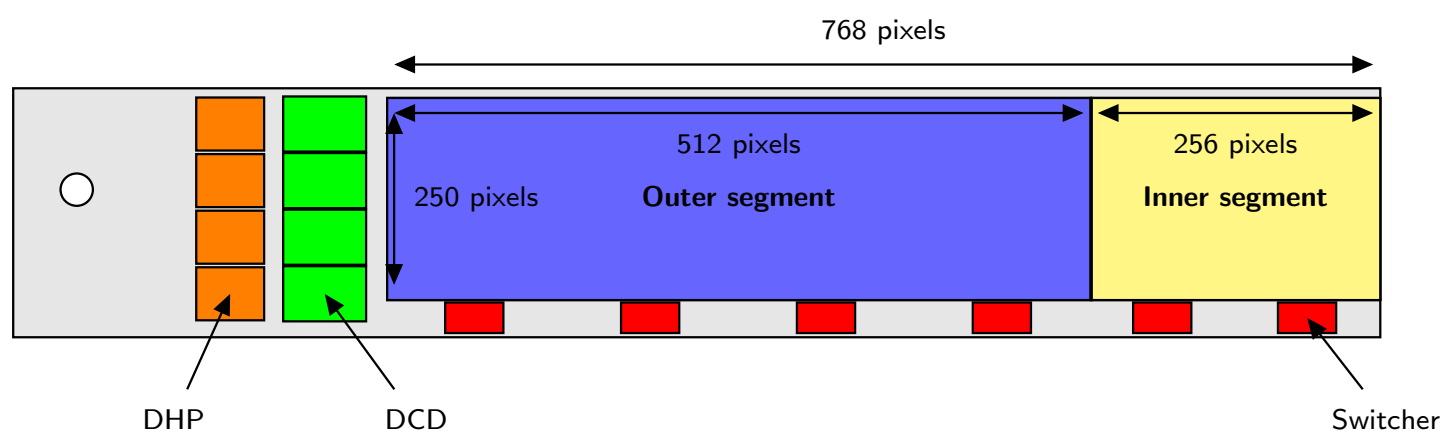

Figure 4.9.: Sketch of a half ladder. The DEPFET matrix is divided into 2 sections, inner segment has smaller pixels than the outer segment. The switchers (red), DCDs (green) and DHPs (orange) from the front-end readout electronic.

of one quarter of the matrix. The long side of the matrix is divided evenly into three parts. The one farthest away from the DHPs/DCDs has smaller pixels than the other two. Each of the three sections has its own Gate-On and CCG line. This design helps to deal with inhomogeneities in the matrix along this axis and is particularly important when radiation damage is considered. In total there are four types of half-ladders used in the PXD: an inner-forward (IF), an inner-backward (IB), an outer-forward (OF) and an outer-backward (OB) type. As their names suggest, they are placed at different positions relative to the interaction point and the electron beam direction of SuperKEKB.

Table 4.1 summarises their mechanical properties. The forward and backward halfladders are glued together before their installation in the vertex detector to increase their mechanical stability. The thickness of the half-ladders is not homogeneous. The 


\begin{tabular}{cccccc}
\hline type & length $[\mathrm{mm}]$ & width $[\mathrm{mm}]$ & inner pixel size & outer pixel size & switcher location \\
\hline IF & 44.8 & 12.5 & $55 \times 50$ & $60 \times 50$ & left \\
IB & 44.8 & 12.5 & $55 \times 50$ & $60 \times 50$ & right \\
OF & 61.44 & 12.5 & $70 \times 50$ & $85 \times 50$ & left \\
OB & 61.44 & 12.5 & $70 \times 50$ & $85 \times 50$ & right \\
\hline
\end{tabular}

Table 4.1.: Mechanical properties of all four PXD half-ladder types.

matrix is thinned and has a thickness of only $75 \mu \mathrm{m}$. The balcony with the switchers and the area where the DCDs/DHPs are located has a thickness of $\approx 500 \mu \mathrm{m}$. The overall material budget of one PXD layer is $0.15 \% X_{0}$.

\subsubsection{DEPFET Matrix}

To construct the pixel detector for Belle II, multiple DEPFET cells (pixels) are placed next to each other forming a $768 \times 250$ matrix [59]. Figure 4.10 shows how this matrix looks like in the case of the PXD. In fact, here one can see the smallest unit of the matrix consisting of four individual pixels that share a common FET gate and a clear gate. The clear gate connects also to the next unit, which is why it is referred to as common clear gate (ccg). Pixels within one column share a common drain, a drain line ending in the readout chip. The source is shared by all pixels. An equivalent electrical circuit of the PXD DEPFET matrix can be seen in fig. 4.11. Because all pixels in one column share a drainline, the readout mechanism has to take this into account. While the matrix has geometrically 768 rows and 250 columns, the electrical mapping is different. Four geometric rows are combined to one electric gate, giving a total of 192 gates $^{2}$. A rolling shutter readout is used, which activates one of these gates at a time. The readout time for the whole matrix (going over all gates) is $20 \mu \mathrm{s}$ ( $\approx 100 \mathrm{~ns}$ per electric gate). Using this method all pixels of one gate can be read out at the same time. During the rolling shutter readout there are three states:

Charge Collection: In the charge collection phase the FET Gate is switched off. The clear contact is set to a low positive voltage. All electrons created in the bulk drift to the internal gate where they are collected. Because the FET is switched off, no drain current is flowing.

Sampling: In the sampling phase, the FET is switched on and drain current flows through it, amplified by the charge in the internal gate. The clear contact is still set to a low positive voltage.

Clear: The gate is switched off and the clear contact is set to a high positive voltage. The electrons are removed from the internal gate and the cycle can start over.

\footnotetext{
${ }^{2}$ These gates should not be confused with the FET gate or the clear gate of a single DEPFET pixel.
} 


\section{Pixel Vertex Detector for Belle II}

It is worth nothing here that the clear process is rather short and therefore often called "clear pulse".

The (negative) depletion voltage applied to the backside of the DEPFET creates an electric field which causes the electrons created in the bulk to drift upwards to the internal gate. The PXD uses a second electric field to create a sidewards drift, simply called drift contact. It is a p-doped region to which a negative voltage is applied. To differentiate it from the backside depletion it is referred to as sideward depletion. Because the voltage is significantly lower than the backside voltage (called $H V$ from here on) the drift of the electrons is slower in this direction.

Overall there are eight voltages needed to operate the DEPFET matrix. Table 4.2 shows all voltages together with their typical values. The optimization of all the voltages is a highly non-trivial task since all of them influence the electric fields within the bulk and thereby the charge collection efficiency. One has to make sure that during the charge collection phase no charge is lost in the clear contact and that the clear process removes all charge from the gate.

As mentioned in section 3.1.1, there is a special accelerator background connected to the continuous injection scheme used at SuperKEKB which produces noisy bunches. The PXD handles this background by a special operation mode called Gated Mode. In this mode the large positive Clear-On voltage is applied together with the Gate-Off voltage (DEPFET transistors disabled). As a consequence the potentials inside the bulk are changed so that newly generated charges do not drift towards the internal gate but directly to the clear contact, i.e. there is no charge collection. Additionally, the potentials make sure that any charge already present in the internal gate is not removed but stays there (suppressed clear). The PXD is switched into Gated Mode when a noisy bunch passes by which is signalled by the accelerator trough a veto signal. A more detailed description of the Gated Mode mechanism can be found in [60].

\begin{tabular}{ccccccccc}
\hline voltage & Gate-On & Gate-Off & Source & Clear-On & Clear-Off & CCG & Drift & HV \\
\hline value & $-2.5 \mathrm{~V}$ & $5 \mathrm{~V}$ & $6 \mathrm{~V}$ & $19 \mathrm{~V}$ & $5 \mathrm{~V}$ & $0 \mathrm{~V}$ & $6 \mathrm{~V}$ & $70 \mathrm{~V}$ \\
\hline
\end{tabular}

Table 4.2.: Summary of all DEPFET voltages together with typical values. During the rolling shutter readout the Gate and Clear voltages switch between their "on" and "off" values to switch between the collection, readout and clear phase of a gate. 


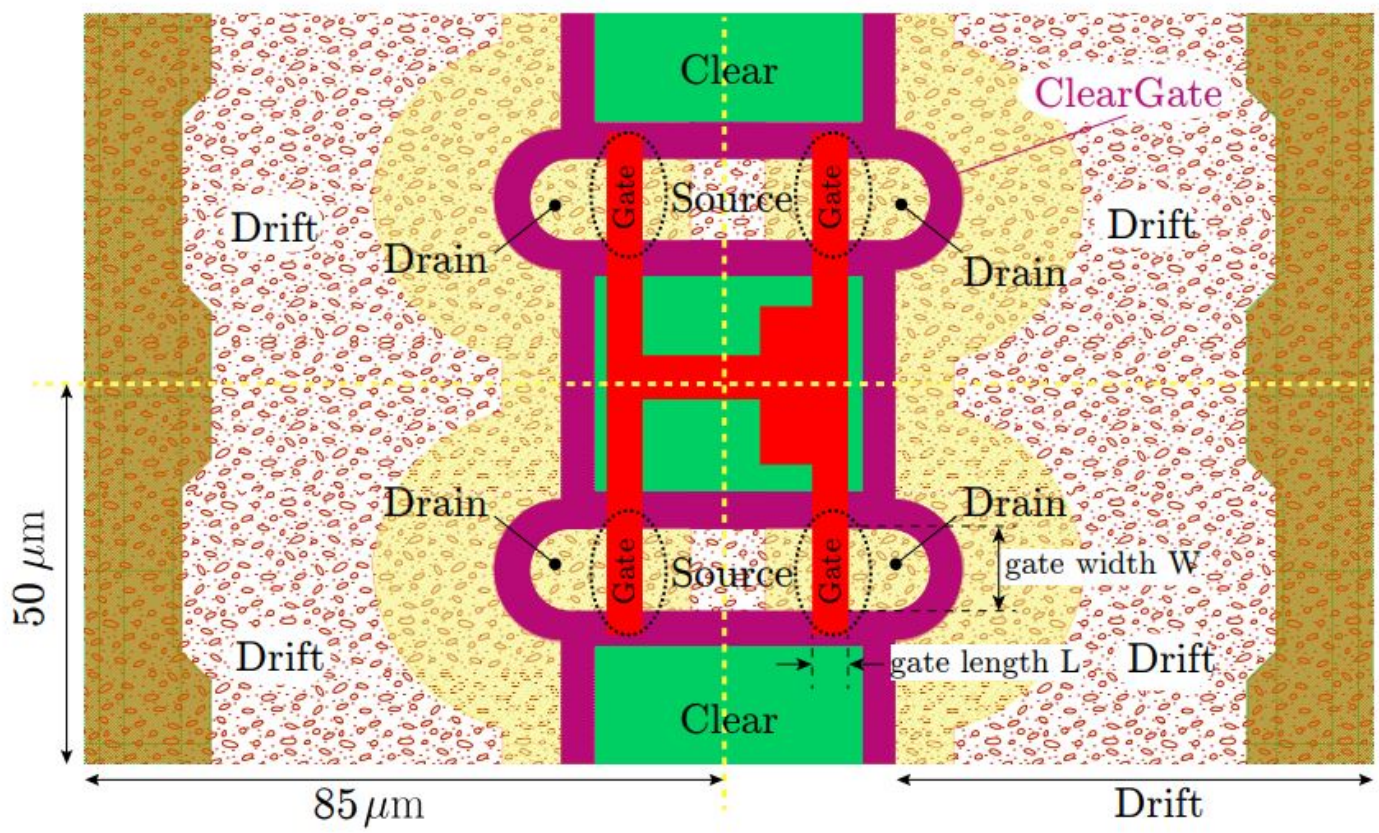

Figure 4.10.: Structure of the DEPFET matrix, from [60].

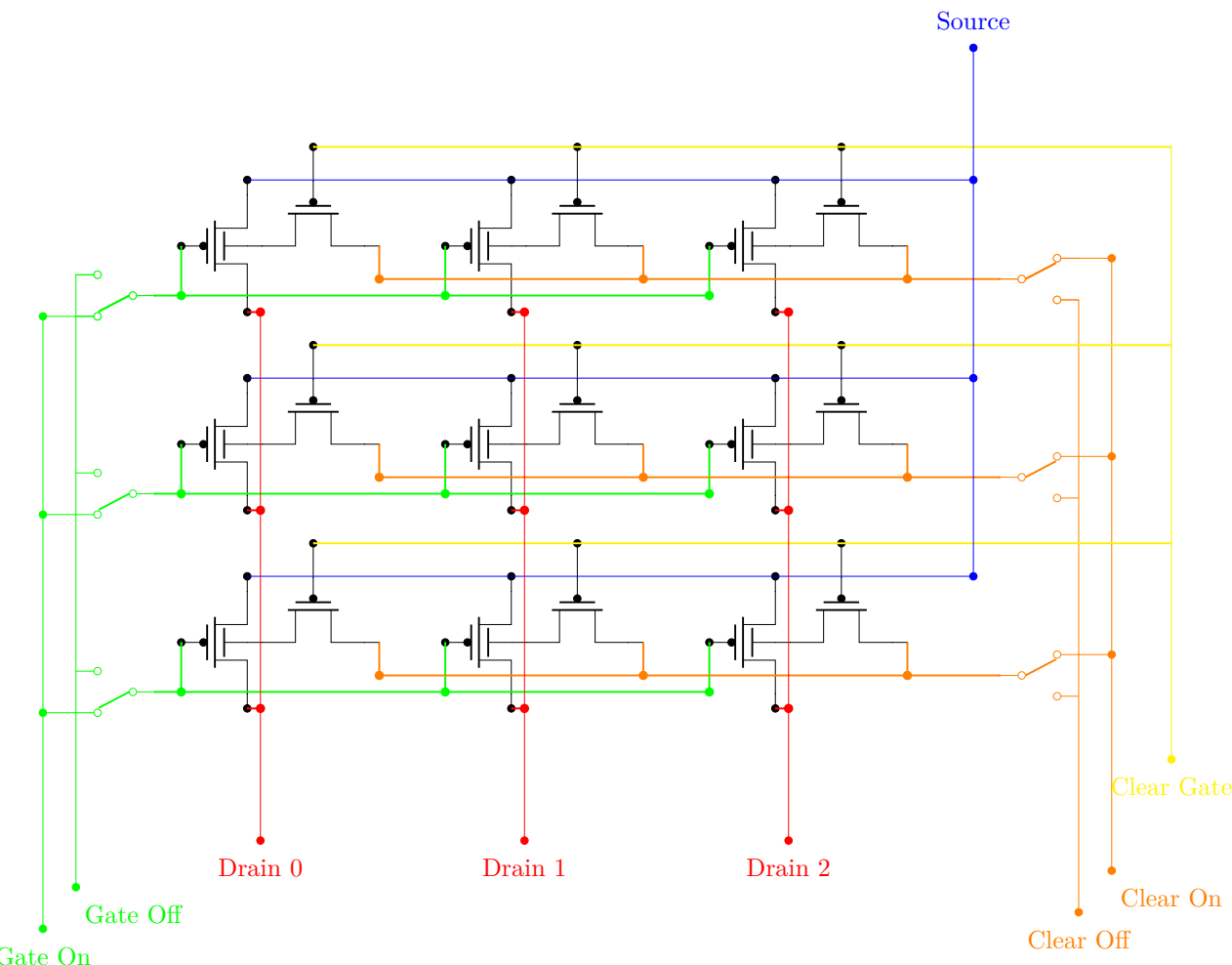

Figure 4.11.: Simplified representation of the DEPFET matrix as an electric circuit. 


\section{Pixel Vertex Detector for Belle II}

\subsubsection{Switcher}

The switcher ASIC is responsible for switching the FET gate and the clear voltage during the rolling shutter. In total there are 6 of them located next to the matrix. Each of them steers 32 electric gates (or 128 geometric rows). They are connected to each other in a row and controlled externally by one of the DHPs on the module. The DHP generates four control signals, also called switcher sequence, that propagate through each of the switchers. The switcher itself needs one voltage $(1.8 \mathrm{~V})$ for its digital part and two

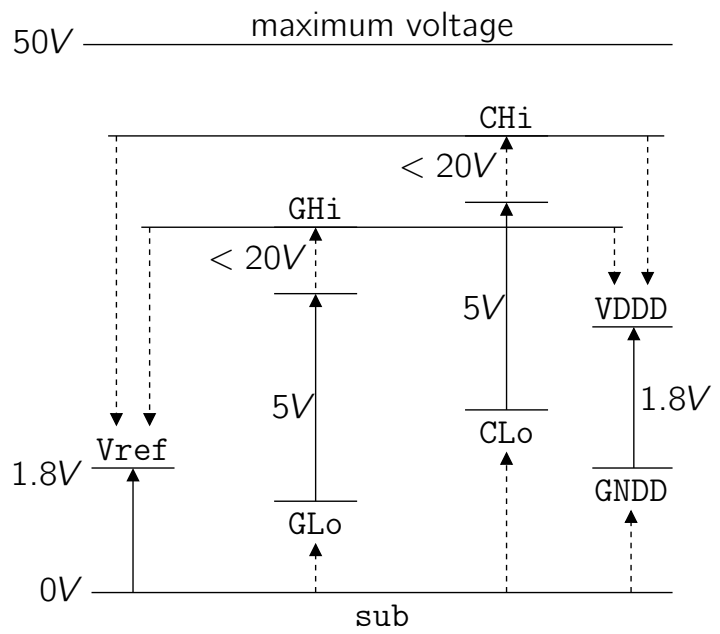

Figure 4.12.: Relation of switcher voltages to each other. $V_{\text {sub }}$ has to be the lowest voltage overall with $V_{\text {ref }}$ being $1.8 \mathrm{~V}$ above it. The difference between $V_{\text {Gate/Clear-On }}$

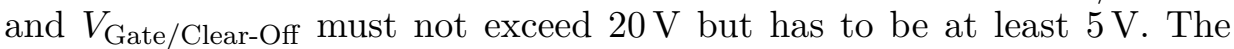
highest voltage must not exceed $50 \mathrm{~V}$.

voltages for its regulators called $V_{\text {ref }}$ and $V_{\text {sub }}$. The latter has to be the lowest voltage on the entire half-ladder at all times, while the former has to be $1.8 \mathrm{~V}$ above it. There are other constraints concerning the voltages of the switcher which are summarised in fig. 4.12 .

\subsubsection{Drain Current Digitizer (DCD)}

The drain current digitizers [57] (DCD) ASICs are located at the end of the matrix and digitise the drain currents of the DEPFETs. During the development phase of the PXD project there have been multiple versions of the DCD. The version used on the half-ladders that are installed in Belle II is called DCD4.2.

Each DCD has the capability to digitise 256 currents in parallel. Of those inputs only 250 are used, resulting in the parallel readout of 1000 pixels in one readout cycle as there are 4 DCDs on each half-ladder. For the digital output to the DHP the DCD has eight 8-bit wide outputs, where each of these outputs is used to transfer the data of 32 channels. To test the communication with the DHP the DCD can be put into 
test pattern mode. In this mode it will output a predefined test pattern which can be checked against to verify a stable communication between DCD and DHP.

\section{Digitization and Parameter Optimization}

The analog to digital conversion (ADC) of the drain currents is done using a redundant signed-digit conversion. The comparison of currents that is needed for this method is done via current memory cells. At the end of the conversion an 8-bit digital code is generated, representing values within the range from 0 to 255 ADU (Arbitrary Digital Unit).

The quality of the digitization process can be evaluated by looking at ADC transfer curves. These curves are generated by feeding a known current into the DCD and checking the digitised result. The are multiple current source available to record the transfer curves. The DCD itself has a built-in current source that can be used for this purpose. Yet, measurements have shown that this current source has a non negligible noise and is therefore not the optimal solution. Another possibility is the use of the DEPFETs themselves and their drain currents. By varying the applied gate voltage $V_{\text {Gate }}$ the drain current of the DEPFETs can be steered to record an ADC curve. The disadvantage of this method is the non-linear dependency of the drain current on the gate voltage. The last possibility is an external current source. An example of such a

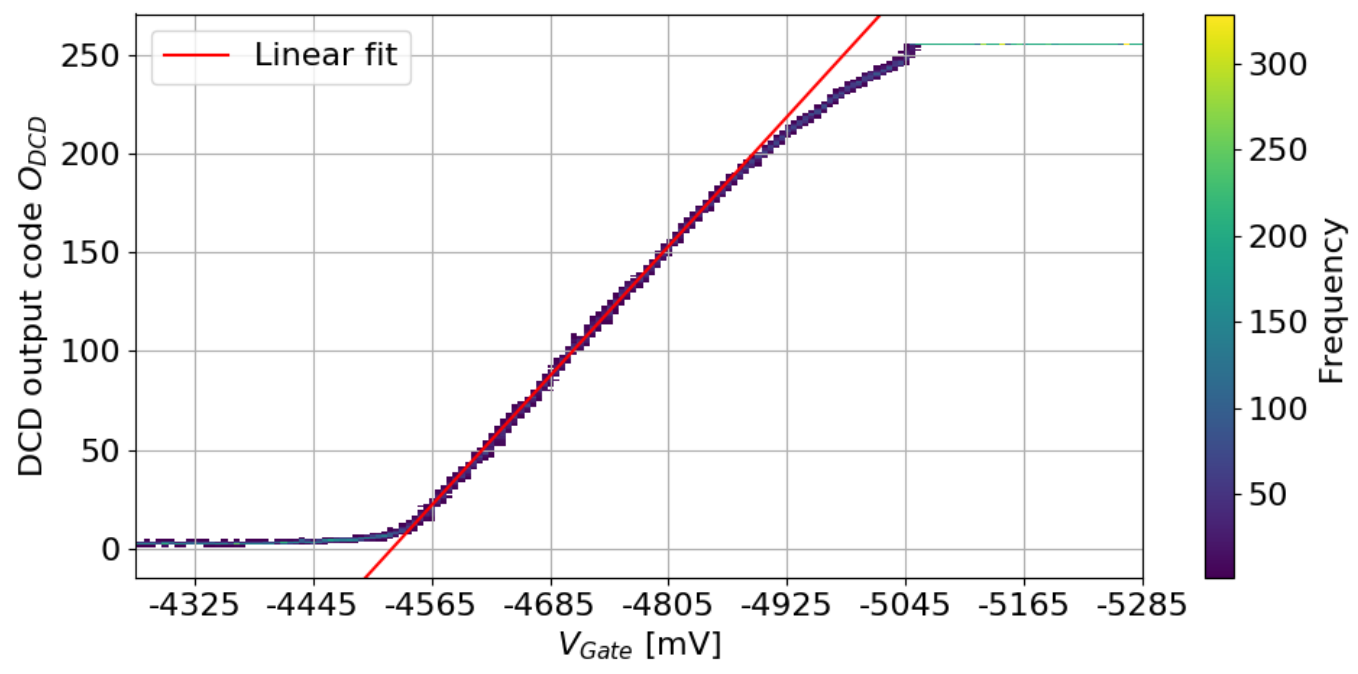

Figure 4.13.: Two dimensional histogram of a recorded ADC transfer curve. The curve was measured by varying the DEPFET gate voltage $V_{\text {Gate }}$ to control the input current into the DCD while recording its response $O_{D C D}$. While the curve covers the whole output range of the DCD and no code jumps are visible, the linearity of the curve for high values of $O_{D C D}$ is sub-optimal as the linear fit shows. 


\section{Pixel Vertex Detector for Belle II}

curve can be seen in fig. 4.13. These curves are used to judge the following criteria: Linearity, dynamic range (min. output code and max. output code), missing codes and noise. "Missing codes" refers to cases where certain output codes are not present in the curve, resulting in gaps within the curve. This error can occur when the current memory cells are not configured optimally. Based on these criteria the curves are categorised as grade-A/B/F. In appendix A more details about the quality criteria and the grading are given together with more examples of ADC transfer curves.

The DCD features several settings to configure the digitization process called DACs. These DACs have to be optimised together with two reference voltages $V_{\text {refin }}$ and $V_{\text {amplow }}$. In total there a five adjustable parameters that have a significant influence on the digitization process of the DCD. For practical reasons the parameter space is sliced to reduce the complexity of the optimization process. Measurements in the laboratory have shown that performing two 2-dimensional and one 1-dimensional scan in the 5-dimensional parameter space reliably produces an setting which fulfils all quality criteria. A comprehensive and detailed description of the optimisation process for DCD can be found in $[61]$.

\section{Common Mode and Offset Correction}

In addition to the pure digitization of the drain currents, the DCD has two additional features built in that improve the overall performance of the system. The first one is the analog common mode correction (ACMC). It uses an analog feedback circuit to remove electronic common mode noise from the drain currents before the digitization is started. During this correction the DCD calculates the mean of all incoming currents and subtracts it from the individual drain currents. Because the DCD digitises one gate after another, the common mode correction is also applied per gate. The ACMC is optional and can be switched off, which is required for certain kinds of calibration measurements.

The second important feature of the DCD is the so-called 2-bit DAC correction. It describes a mode of operation in which the DCD adds a predefined offset current $I_{D A C}$ to the input current $I_{i n}$. This offset current can be set for each drainline and conversion cycle individually, allowing a per pixel correction. While the size of the offset current is set globally, the effect on each pixel can be adjusted in four levels:

$$
\begin{aligned}
& 0: \quad I=I_{\text {in }}+0 \times I_{D A C} \\
& \text { 1: } \quad I=I_{\text {in }}+1 \times I_{D A C} \\
& 2 \text { : } \quad I=I_{\text {in }}+2 \times I_{D A C} \\
& 3: \quad I=I_{\text {in }}+3 \times I_{D A C}
\end{aligned}
$$

This allows to decrease the width of the input current distribution significantly. The input current distribution is divided into four parts of equal width. The highest setting $\left(3 \times I_{D A C}\right)$ is applied to the lowest part of the four while the second highest is applied to the second lowest part and so on. Figure 4.14 shows a graphical sketch of this procedure. Because the DCD does not have a memory to hold the information for which 


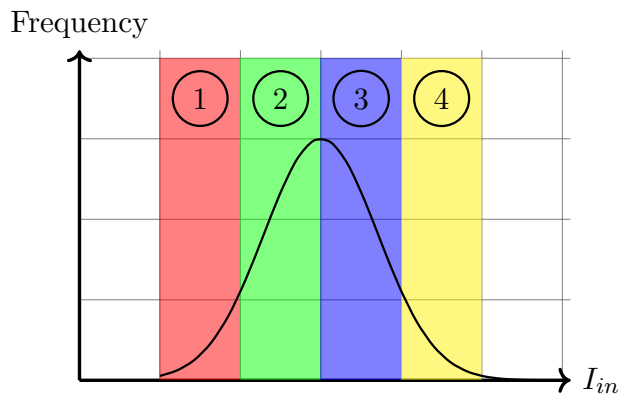

(a) before

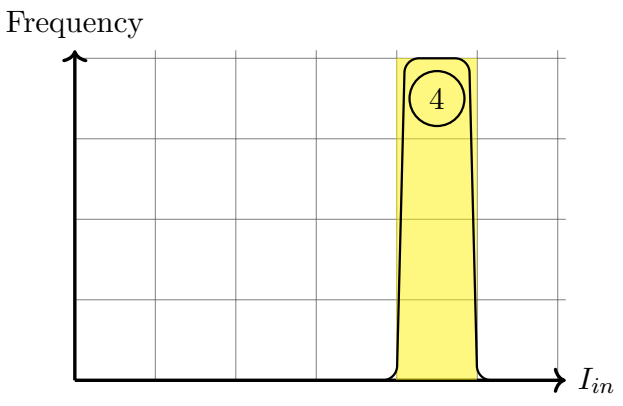

(b) after

Figure 4.14.: Idealized representation of the offset correction principle. The pedestal distribution of the sensor (typically Gaussian) is divided into four parts (a). The pixels belonging to the three lowest parts are shifted by adding a current from the DCD. Ideally the resulting distribution has a four times smaller width (b).

pixel which correction has to be applied, this information is stored in the DHP memory. The DHP sends out a control signal to the DCD during each digitization cycle to steer the correction.

\subsubsection{Data Handling Processor (DHP)}

The data handling processor (DHP) receives the data from the DCD and processes it further. Its main task is the data rate reduction. The incoming data rate (from the DCD) is approximately $20 \mathrm{~Gb} / \mathrm{s}$, the outgoing data rate is limited by the back-end electronics to a value of $\approx 1.6 \mathrm{~Gb} / \mathrm{s}$. To reduce the data rate the DHP performs a zero suppression on the incoming data. Only the signals from pixels that are above a defined threshold are sent out to the back-end electronics. With this approach only "hits" on the matrix are sent out.

\section{DCD-DHP Communication}

As described in section 4.5.3, the communication between the DCD and the DHP is realised by eight 8-bit wide data links. To ensure a stable and error-free data transmission, the sampling point on these 64 data lines can be adjusted on the DHP side. The DHP has dedicated delay elements (also called local delay elements) for each of the data lines in conjunction with delay elements that affect all data links simultaneously (called global delay elements).

Up to 15 of these elements can be connected in series to create a longer delay on a single line or globally. The DHP has registers to adjust the number of delay elements. An optimisation of these settings is necessary to ensure the DHP's sampling point is correct for all data lines. For this optimisation the DCD is put into a test mode, in which 


\section{Pixel Vertex Detector for Belle II}

it outputs a constant pattern instead of digitised currents from its inputs. The same pattern is sent over all eight links. During one readout cycle each link sends the data for 32 pixels. The test pattern is designed to have many bit changes during the 32 clock cycles and also to test for cross-talk between the individual data lines. By comparing the expected data to the received data it is possible to look for bit errors for the different combinations of local and global delay settings. Figure 4.15 shows an example of a scan over the delay parameter phase space for a single link. During the optimisation a set of parameters has to be found that reduces the number of observed bit errors to zero for all 64 links.

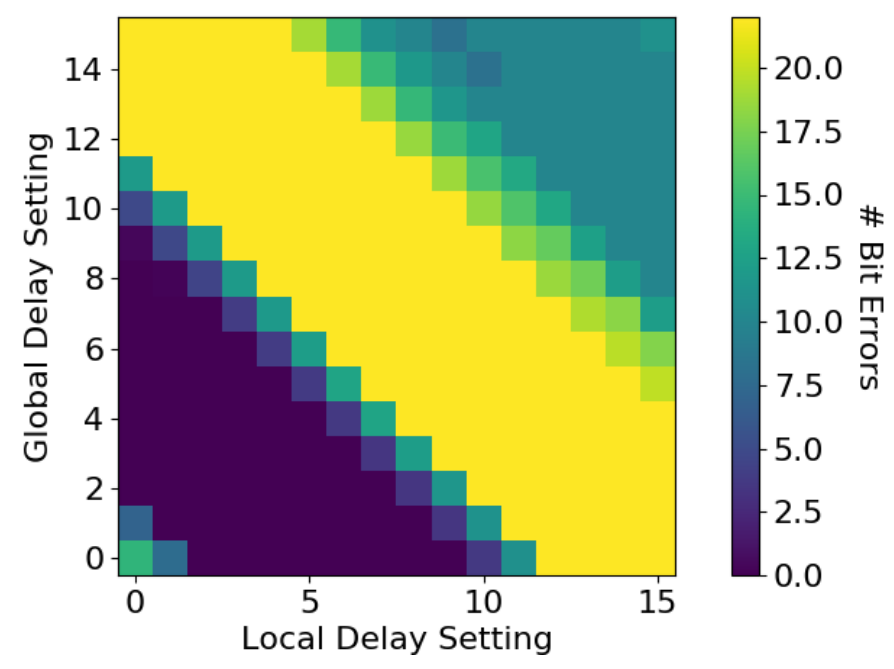

Figure 4.15.: Example of a delay optimisation scan for a single link. The $\mathrm{x}$-axis shows the number of local delay elements put in series and the $y$-axis the number of global delay elements. The colour shows the average number of bit errors observed in 100 data frames. The blue band shows the error-free region in the parameter space.

\section{Data Processing}

The DHP knows two basic modes of operation: raw data mode and zero suppression mode. The latter is used for normal operation of the experiment while the former is used to calibrate or debug the system. In raw data mode the incoming data is sent out without any further processing. Therefore, the data rate is not reduced and the trigger rate of the system is limited significantly. The zero suppressed readout is more sophisticated and divided into multiple steps.

Pedestal subtraction: The first step is the subtraction of the so-called pedestals. As mentioned in section 4.3 the drain current of a DEPFET is influenced by the 
charge in the internal gate. Even if there is no charge in the internal gate there is still a non-zero drain current flowing through the FET. Because of the production technique (e.g. variations in the doping) this "zero-charge" current is not identical for all FETs. The collection of all these currents is referred to as pedestals. To identify those pixels with a "hit" (charge in the internal gate) it is therefore necessary to subtract the known pedestals first.

Digital Common Mode Correction: The next step is the (optional) digital common mode correction (DCMC). Like the DCD, the DHP is capable of eliminating electrical common mode noise. Instead of a feedback circuit a purely digital removal is done using a technique called two parse average (TPA). In the first step a rough common mode estimate $\mathrm{CM}^{*}$ is made by calculating the average of all input data values $I_{j}$ :

$$
\mathrm{CM}^{*}=\frac{1}{N} \sum_{j} I_{j}
$$

In the second step all input values are checked against this value and replaced by it if they are above the value plus a predefined threshold $t_{D H P}$ :

$$
\mathrm{CM}=\frac{1}{N} \sum_{j} \begin{cases}I_{j} & I_{j}<\mathrm{CM}^{*}+t_{D H P} \\ \mathrm{CM}^{*} & \text { otherwise }\end{cases}
$$

The final value $\mathrm{CM}$ is then used and subtracted from all data values in order to remove the common mode noise.

Hit finding: In the last step all data values are compared to the global hit finding threshold $t_{\mathrm{Hit}}$. Only those values above the threshold are sent out to the backend electronics. Typical values for the threshold are $5-7$ ADU depending on the overall noise of the system.

\section{DHP Memories}

The DHP has several memories to store the received data, the pedestals, the switcher sequence and the 2-bit offset data. The main memory has 16 memory blocks and each block consists of 1024 words, where each of the words is 128 bit long. Each of the memory blocks is structured in the same way. The first 512 words are reserved for the data values. This is enough space to store $\approx 2.66$ full data frames ${ }^{3}$. The other half of a memory block is reserved for the pedestal data. This part is further subdivided into two parts which allows to store two sets of pedestals and quickly switch between them.

For the 2-bit offset correction values there are two additional memory blocks with the same specifications as the main memory. The memory is large enough to store the data

\footnotetext{
${ }^{3}$ Each word can store the data for 16 pixels and each DHP processes $192 * 256$ pixels during one frame. $\frac{16 \times 512 \times 16}{192 \times 256} \approx 2.66$
} 


\section{Pixel Vertex Detector for Belle II}

for each of the $768 \times 250$ pixels so that the DCD can perform a proper pedestal compression.

The last memory is the switcher sequence memory. It is a single memory block with 1024 words. Each word contains the sequence for one gate. The first 512 word are reserved for the normal operation, while the remaining words are reserved for the so-called gated mode operation, which is a special mode of the system that requires another switcher sequence.

\section{DHP Temperature Sensor}

The DHP also features a temperature sensor that can be read out to monitor the temperature of the system. To trigger a readout, certain registers of the DHP have to be set in the correct order via JTAG. However, the measured temperature depends on the applied DHP voltage, which has been verified by measurement in the laboratory. Therefore, a calibration of the sensor is required if the applied voltage is not the nominal one. In any case, the sensor can be used to identify relative changes in the temperature.

\subsection{Back-end readout and Data Acquisition (DAQ)}

The data handling hybrid (DHH) system forms the backend of the PXD readout and is an essential part of the PXD data acquisition. Apart from receiving the data stream from the front-end electronics it also generates the control signals required to configure and trigger the ASICs on the PXD half-ladders. This section gives an overview of its most important components and their functions. A detailed description of the full DHH system can be found elsewhere [62]. A simplified version of the DHH system is also used in the laboratory for the readout of a single half-ladder.

\subsubsection{DHH Components}

The DHH System has three main components. Following the readout stream the next part in the chain after the DHP is the DHE (Data Handling Engine), a readout board based on a Xilinx Virtex-6 LX130T field-programmable gate array (FPGA). All four DHPs of one half-ladder are connected to one DHE with highspeed data links using the Aurora Protocol with 8B/10B encoding. The DHE sends the trigger signal to the DHPs, receives their data and merges it into one DHE data frame.

The DHI (Data Handling Interface) works in parallel to the DHE and is responsible for the configuration of the ASICs on the half-ladder using the JTAG protocol. Prototype versions of the DHE used in the laboratory combined the functionality of the DHI and the DHE on one FPGA board which was also referred to as DHE. Figure 4.16 shows a single DHE/DHC unit. The units are built in the advanced mezzanine card (AMC) form factor. These units are mounted on carrier board to be operated in a stand-alone manner (laboratory) or together with other units in an ATCA (Advanced Telecommunications Computing Architecture) shelf. 


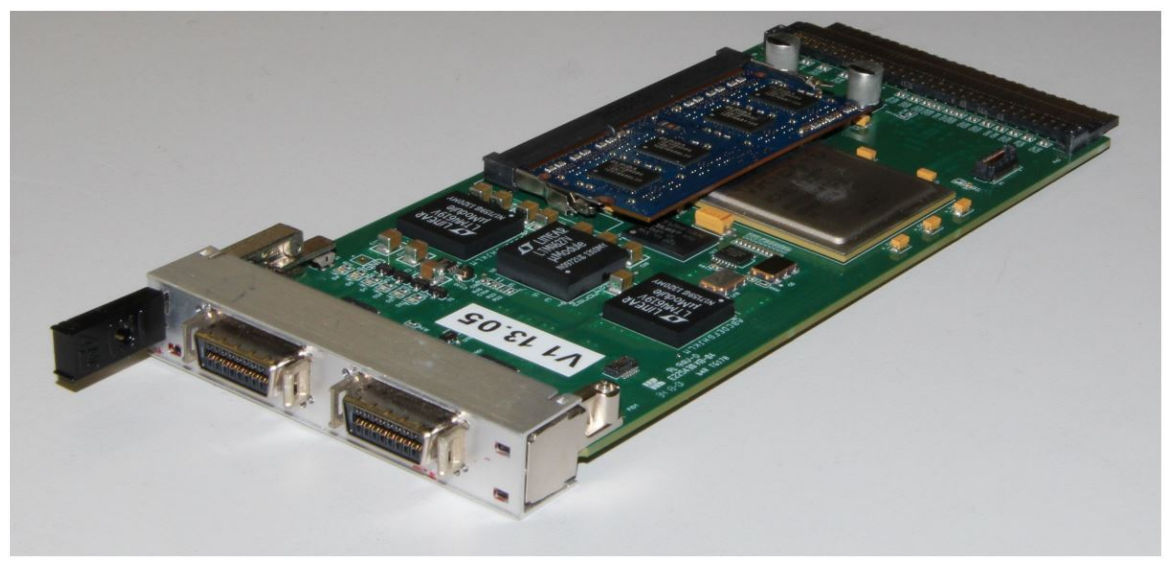

Figure 4.16.: Photograph of a DHH module (either DHE or DHC). The unit shown here has additional DDR3 RAM attached (blue card) to buffer incoming data and function as a DHC. From [62].

The data from 5 DHEs is collected in one DHC (Data Handling Concentrator). The DHC merges the 5 DHE data frames into a single DHC frame before it is send out further. The inner PXD sensors (and thereby the inner DHEs) are expected to have a higher data rate since they are closer to the interaction point where they are effected more strongly by the background than the outer ones. If the inner and outer sensor are distributed to the DHCs in the right way, the data rate of the DHCs can be averaged, making the subsequent data processing and load balancing easier.

\subsubsection{Full DHH System and Global DAQ Integration}

The DHH system functions as the connection of the PXD system to the rest of Belle II. Apart from transmitting the received data to the global Belle II DAQ system, it also ensures that the PXD is synchronised with the SuperKEKB accelerator by propagating the signals from the Belle II trigger and time distribution system (B2TT) [63]. From the B2TT clock (127.21 Mhz) the DHH system generates the $76.33 \mathrm{Mhz}$ clock that is further propagated to the ASICS on the PXD half-ladders.

The full DHH system consists of 40 DHE/DHI pairs and 8 DHCs to process the data of all 40 PXD half-ladders. Even with the zero-suppression of the DHPs the data rate of the PXD is still too high for the global Belle II Data acquisition system (Belle II DAQ). Assuming a trigger rate of $30 \mathrm{kHz}$ and an occupancy of $3 \%$ (which is the maximum possible occupancy for the DHP), the data rate of the full system is $\approx 22 \mathrm{~GB} / \mathrm{s}$. To reduce the data rate, the DHCs send their events to the ONSEN system [64], where they are buffered.

The Belle II DAQ system uses data from all subsystems but the PXD to construct an initial event when the (low level) hardware trigger is activated. The events are passed to the Belle II high level trigger (HLT) system [65] (high performance parallel computer farm), where a first tracking procedure is started. The reconstructed tracks 


\section{Pixel Vertex Detector for Belle II}

are extrapolated to the PXD sensors and a region of interest (ROI) around the predicted hit position is determined. These ROIs are passed together with the corresponding event numbers to the ONSEN system, which filters the PXD data and returns all registered PXD hits within the ROI of the given event. The data rate reduction factor because of the high level trigger and the ROIs is around 30 [64]. Together with the added PXD information a second event building process (including a new track reconstruction with the PXD data) is started before the data is stored to disk. Figure 4.17 shows a sketch which summarises this workflow.

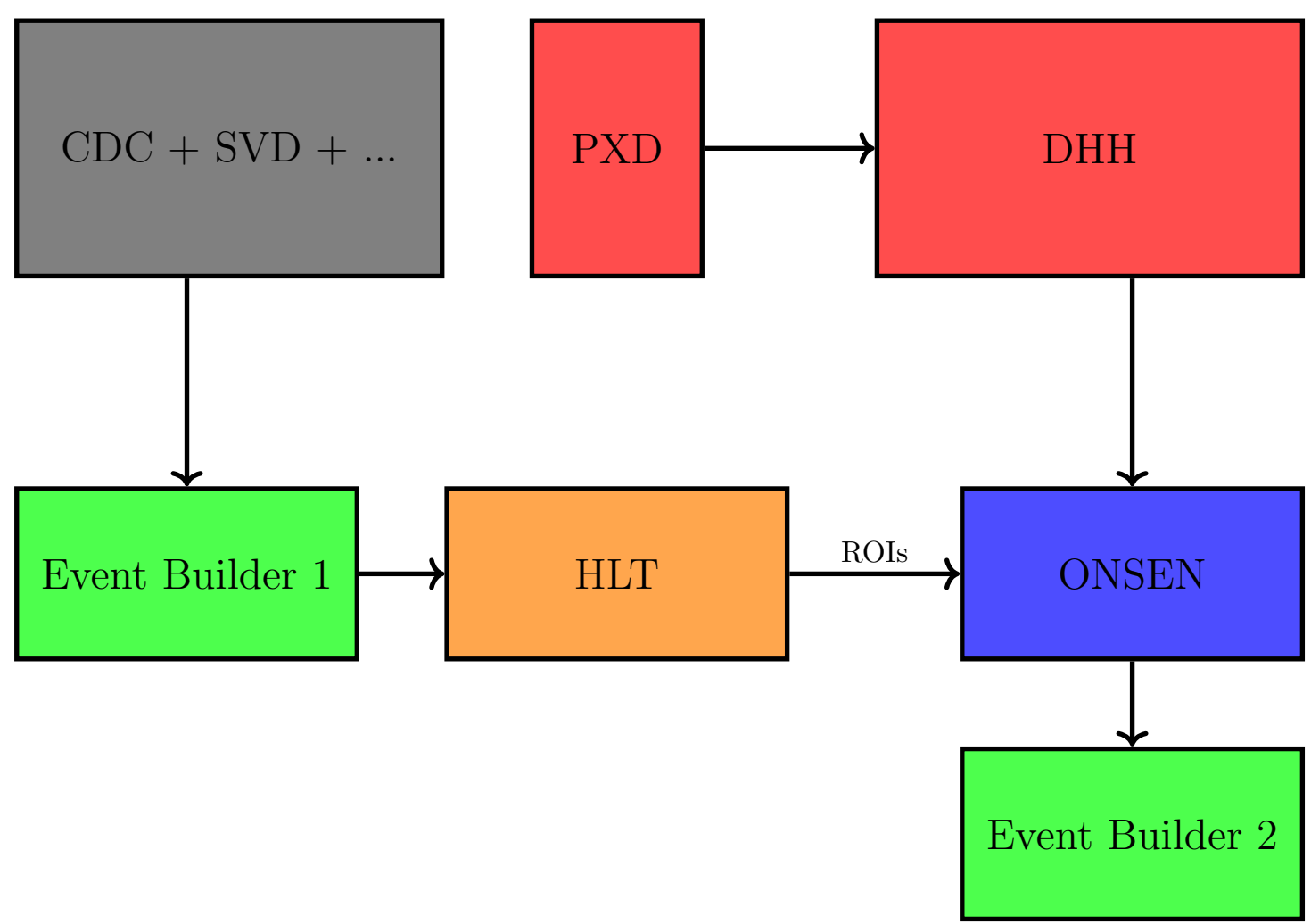

Figure 4.17.: Sketch of the dataflow of the Belle II DAQ system. PXD data is not used in the first reconstruction of an event but buffered and reduced in the ONSEN system. In a second event building step the PXD data is added to the global data stream.

There is a second outgoing data stream from the DHH system which sends a copy of the raw PXD data out to a connected computer using UDP (User Datagram Protocol). This data stream is used for the local PXD DAQ, which is used for data quality monitoring but also serves as the main data source for calibration measurements of the PXD. It is also the DAQ system that is used in the laboratories. 


\subsubsection{Local PXD DAQ and Laboratory Readout}

In the laboratory a simplified DHH system can be used to operate a half-ladder. In this configuration the DHC component is missing and the DHE (with DHI functionality) is directly connected to a control computer. The data transmission is realised via UDP packages that are sent over a RJ45 cable. These UDP packages are are received and processed by a custom made local PXD DAQ developed in Bonn [66].

To read out the sensor without an external trigger source, the DHE can generate its own triggers in this configuration. It is however still possible to attach an external trigger device to the DHE carrier board and use these external triggers.

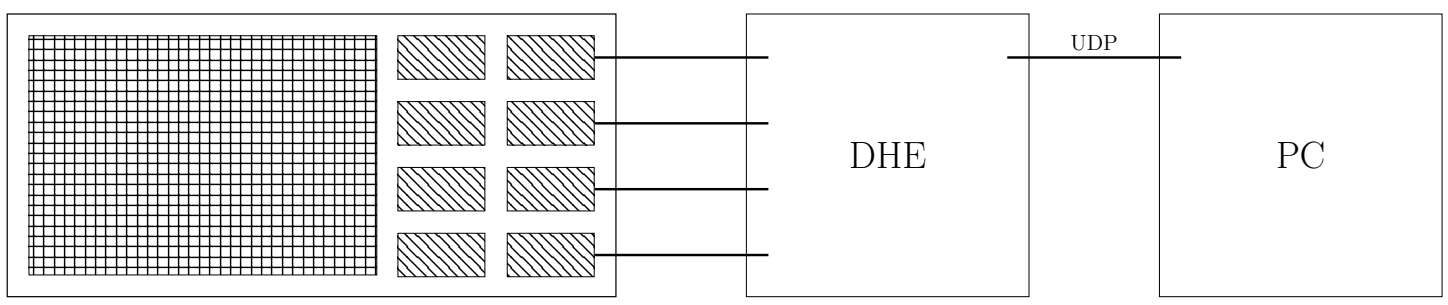

Figure 4.18.: Simplified Laboratory Readout scheme of a single PXD half-ladder. The DHPs are connected with high speed data links to the DHE, which send out its data frame via UDP to a connected PC.

This simplified readout scheme (see fig. 4.18) allows to test (partially) the DHH functionality without the need to simulate the entire Belle II DAQ system. Instead of a pure DHE based readout a DHE+DHC system can also be used in the laboratory to test the DHC functionality.

\subsection{Power Supply and Sevices}

Operation of a PXD sensor requires various services that will be presented here shortly. The most important one is the custom power supply developed at LMU (LudwigMaximilians-Universität München), referred to as LMU power supply [67]. Each PXD sensor requires more than 20 different voltages to be operated, including the ASIC supply voltages and the voltages required to bias the DEPFET matrix. These voltages need to be controllable independently and must be ramped in a specific order for the power-up and power-down procedure of a sensor.

The LMU power supplies fulfil these requirements and are used in the laboratory as well as in the Belle II experiment to power the PXD sensors. Figure 4.19 shows a photograph of a LMU power supply unit as it is used in the laboratory. On the front side two connectors are placed to transmit the power for the digital parts (ASICs) and the analog part of the connected PXD sensor. The units are connected to a computer via RJ45 cables, from where they are controlled through an input-output controller software. Each PXD sensor requires its own power supply, therefore 40 power units are needed for the Belle II operation. 


\section{Pixel Vertex Detector for Belle II}

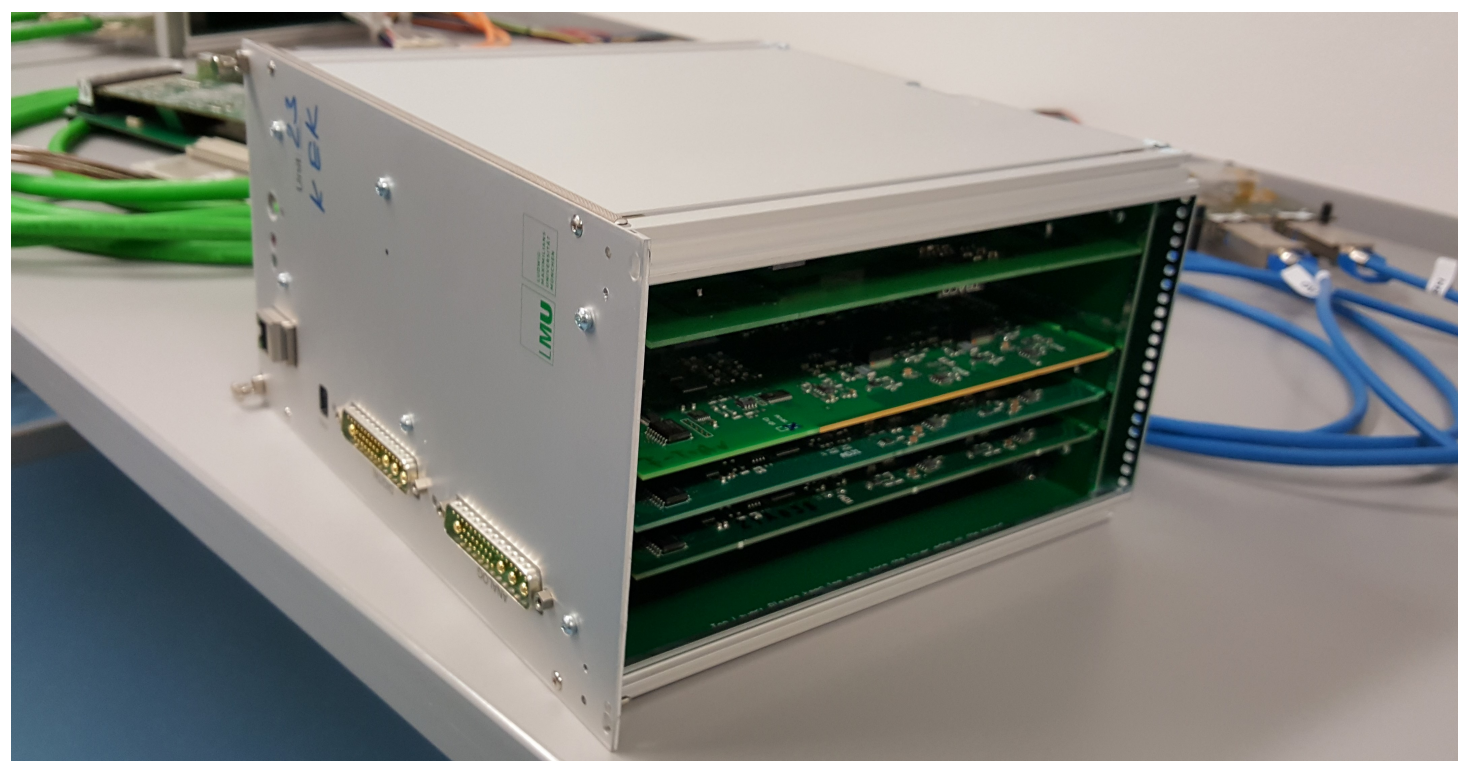

Figure 4.19.: Photograph of an LMU power supply unit.

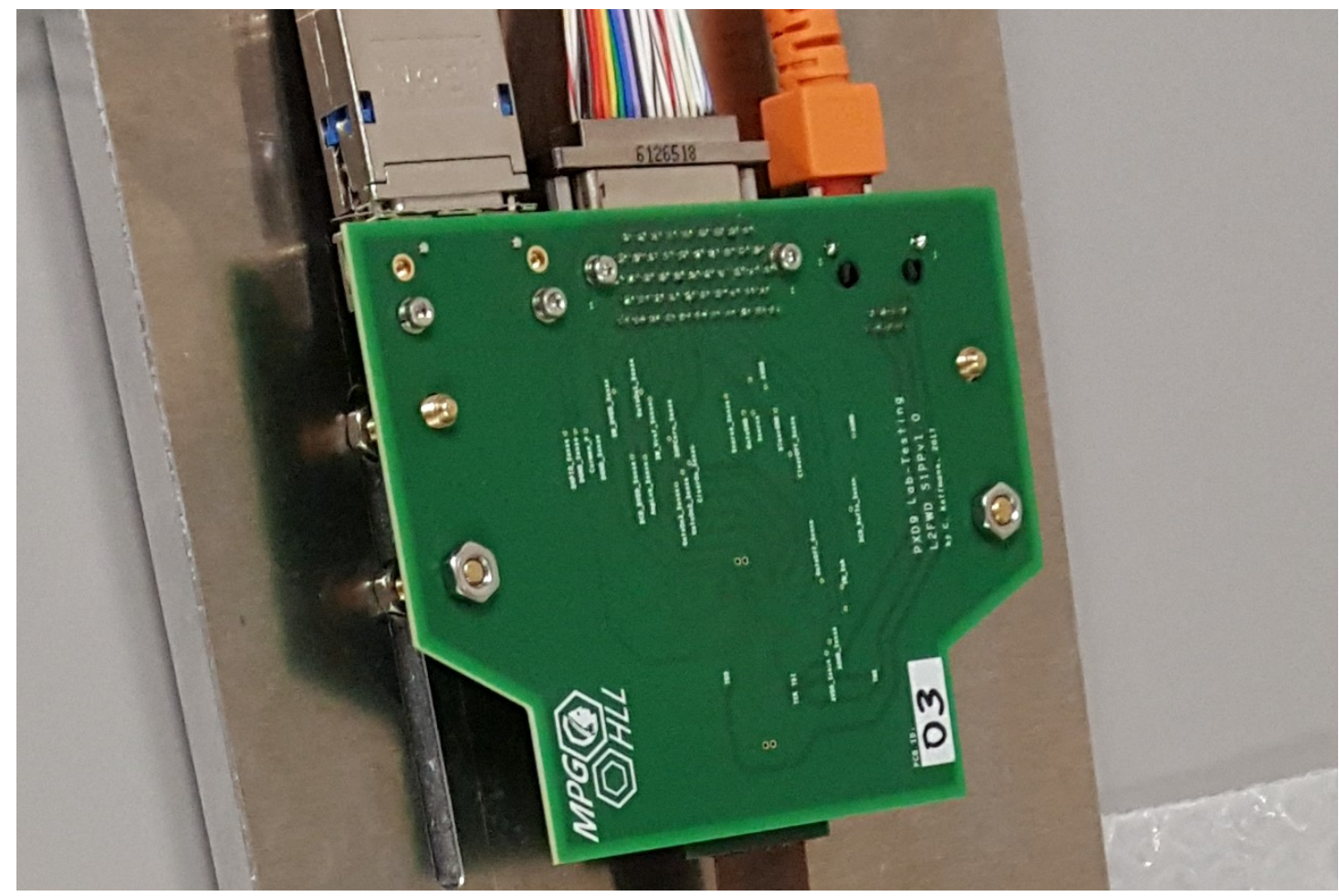

Figure 4.20.: Photograph of a laboratory-type patch panel. 
Connections between the individual components of the PXD (power supply, DHE, half-ladder, ...) are made through intermediate PCBs which concentrate data/power lines and translate signals if necessary. The kapton flex cables of the PXD half-ladders are connected to so-called patch panels. While the exact design of the patch varies between the ones used in the laboratory and those used at Belle II, their functionality is identical. They concentrate the incoming data, power and control lines which are further propagated over the kapton flex cable to the half-ladders. Figure 4.20 shows a laboratory type patch panel. The figure also shows the three cables that are combined by the patch panel: a power cable with a Glenair connector, an Inifiband cable for the data transmission and a RJ45 cable for the control signals.

In the laboratory the data and control lines are directly connected to the DHE unit and the power cable is connected to the LMUPS over a second PCB (power breakout board). At Belle II the data, control and power lines are routed together using another type of PCB (dock box) from where they are further distributed to the LMUPS units and the DHH system. In contrast to the laboratory, the dock box PCBs translate the electronic data signals (Infiniband cable) to an optical signal before it is transmitted to the DHH system.

\subsection{Hybrid5 Sensors}

In addition to the PXD half-ladders with 4 DHP-DCD pairs and a $768 \times 250$ pixel matrix, smaller test boards were constructed during the development of the PXD system. One of these boards is the so-called Hybrid5. Hybrid5 sensors feature only one DHP-DCD pair and one Switcher ASIC. They can be equipped with a small DEPFET matrix that has $64 \times 32$ pixels. Figure 4.21 shows a photograph of a Hybrid5 board with a DEPFET matrix attached to it. The main advantage of these units is the reduced complexity (fewer ASICs) while at the same time the full read-out and operation procedures can be tested. In contrast to a PXD half-ladder the system does also not require active cooling to be operated. 
4. Pixel Vertex Detector for Belle II

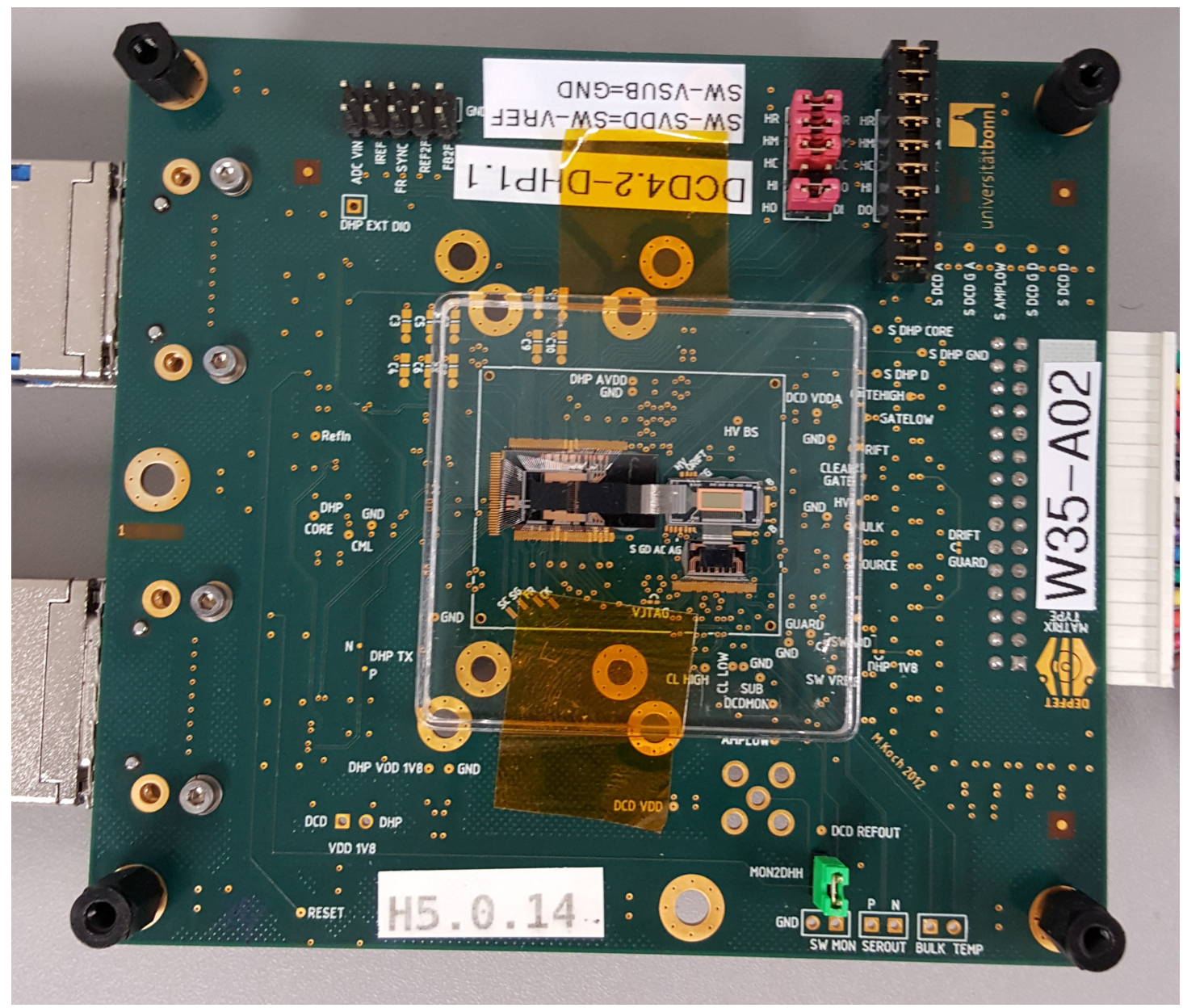

Figure 4.21.: Photograph of a Hybrid5 sensor with a small DEPFET matrix attached. 
CHAPTER 5

\section{Slow Control and Calibration Software}

The previous chapter has focused on the various hardware components that are used for the PXD. How to control and configure the ASICs was only briefly mentioned so far. This chapter will give more information on that subject which is referred to as slow control. This chapter will also give an overview of the calibration strategies for the PXD that are used in the final experiment as well as in the laboratories from a conceptual and a software perspective. Lastly, an automated approach for logging of calibrations and data taking in general will be presented.

\subsection{EPICS}

The Experimental Physics and Industrial Control System (EPICS) [68] is a free software framework designed to control and monitor hardware components in large networks in an easy and reliable way. EPICS is developed by an international team and used worldwide in many laboratories and research facilities. EPICS uses a server/client structure to allow many computers in one network (clients) to connect to dedicated servers to get information about the connected hardware. As these servers typically communicate directly to a hardware object, they are also called Input Output controllers (IOCs). These IOCs contain the drivers to communicate with the attached hardware and provide monitors and control options for the clients.

Each IOC provides PVs (process variables) which (usually) represent an observable of the hardware. They have a name, a value and can also hold information about the unit of the observable. In addition, they provide an alarm status and can contain a predefined range of "allowed" values. This allows to define a range of acceptable or expected values for a voltage and automatically trigger an alarm if the value of the voltage leaves the defined range. 


\section{Slow Control and Calibration Software}

Clients can interact with these PVs by simply knowing their name which serves as an ID. EPICS handles the underlying network traffic by itself. This is done via so-called channel access (CA). A graphical illustration of the CA principle can be seen in fig. 5.1. The underlying protocols used by EPICS for the channel access are UDP as well as

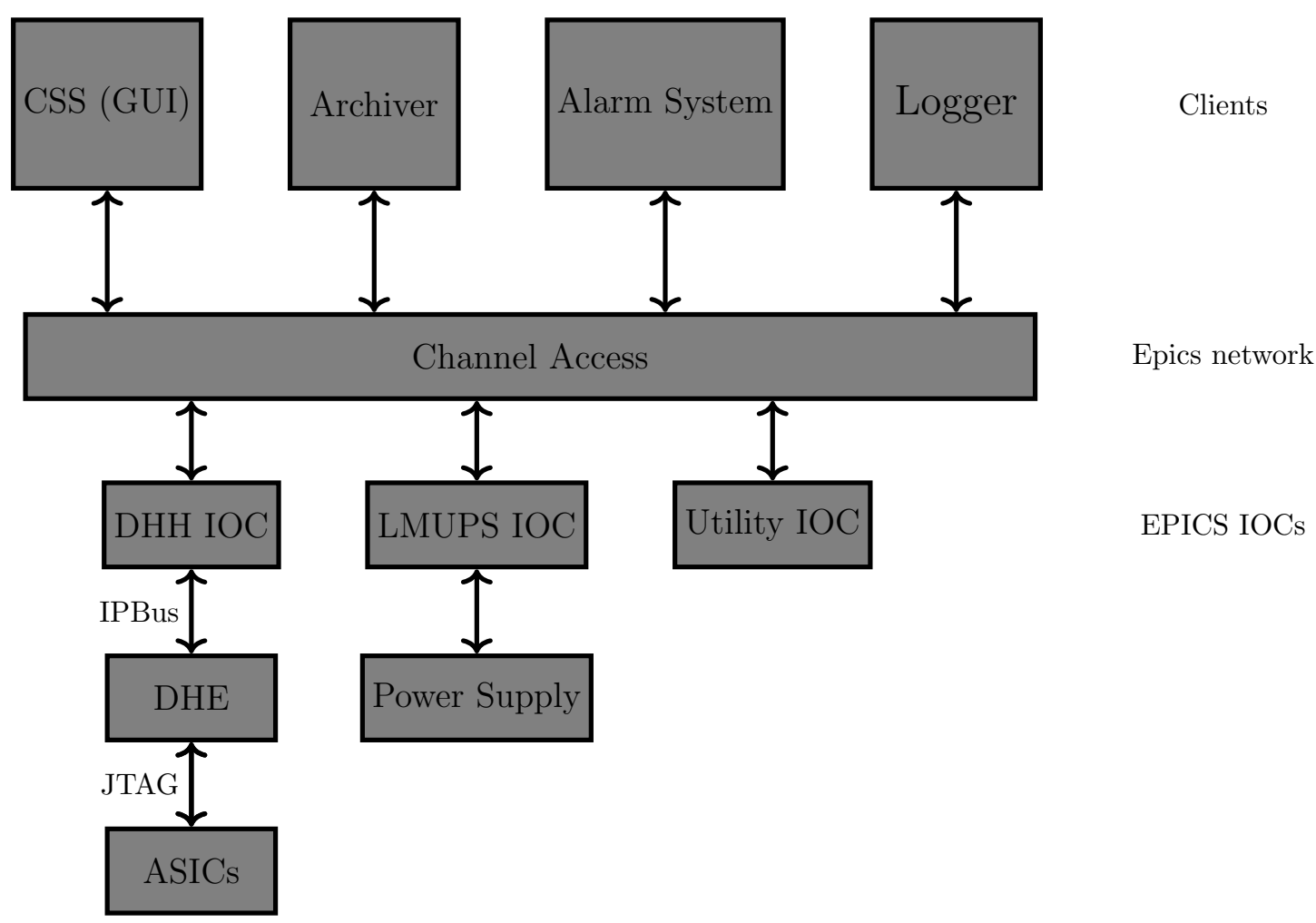

Figure 5.1.: Illustration of the channel access principle used by EPICS. Clients interact over PVs (channel access) in the EPICs network with IOCs which translate the high level commands to signals for the attached hardware. The protocol used in the hardware interactions varies from IOC to IOC. Certain IOCs perform no hardware interaction at all (Soft IOCs).

TCP. Whenever a client tries to connect to a new PV for the first time a UDP search request is sent to the network, to which the corresponding server replies with another UDP package before a permanent TCP connection between the client and the server is established. Each server sends a "beacon" in regular intervals which serves as a heartbeat for the clients that have any TCP connections to the server. If the "beacon" is not received because the server is no longer available, the client tries to reconnect and eventually reports a disconnection from the PV.

To allow a simple access and control of the PVs there are many graphical interfaces (GUIs) available. These GUIs are EPICs clients that connect to PVs via the EPICs 
network and visualise the PVs. The one used by the PXD collaboration and Belle II is Control System Studio (CSS) [69]. It allows the creation of customised widgets that help to visualise the values of PVs and set values to them. Figure 5.2 shows an example of such a widget. These widgets are particularly helpful as they allow non-experts to

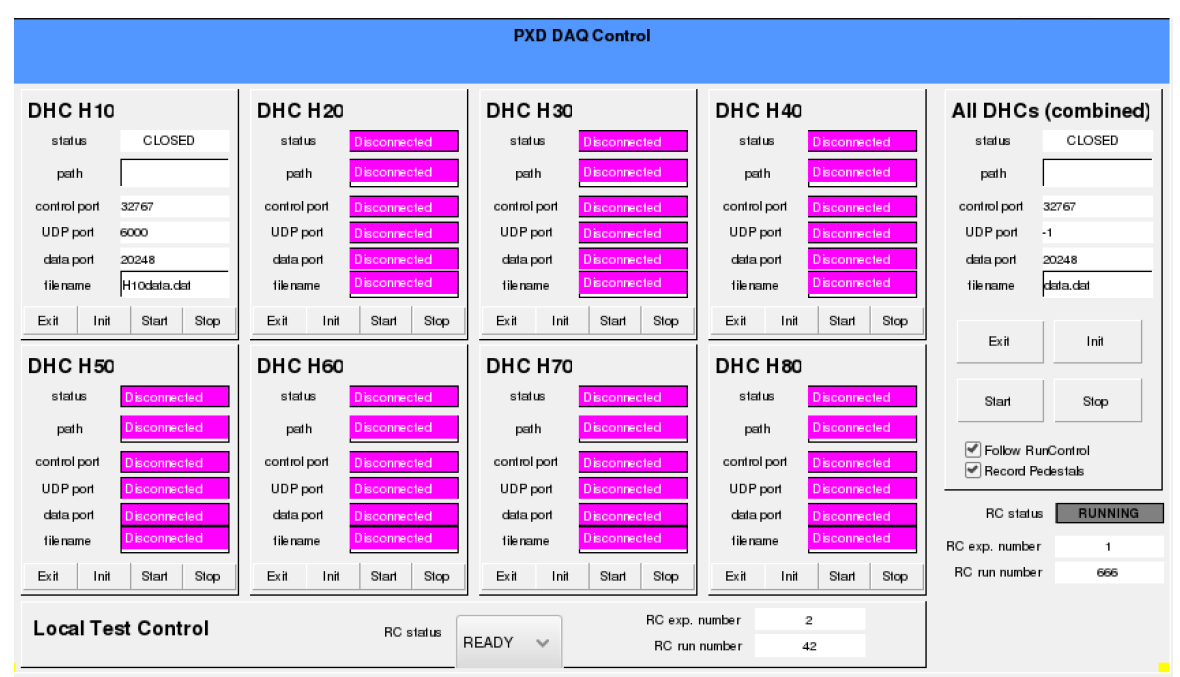

Figure 5.2.: Example of a graphical interface used for the PXD.

operate a complicated system like the PXD in an easy way with safety mechanisms that prevent improper handling of the system.

There is one other important aspect of the EPICS system which is the possibility to $\log$ values of PVs over time. Similar to the graphical interface there are multiple implementations of EPICS archivers. Belle II uses the EPICS Archiver Appliance [70], which allows to $\log$ and archive the values of millions of PVs. This archiver system is not only used for the full Belle II PXD system, but also in the laboratories during the testing and calibration of the half-ladders and prototypes. Access to the archiver is possible via a web interface or directly over CSS. The archiver also provides a variety of metrics and reports to analyse the evolution of one or many PVs.

To operate a single PXD half-ladder several IOCs are necessary. Two of them are communicating directly to the hardware while the others are "soft" IOCs that provide PVs and interact with other IOCs, but do not control hardware directly. The two hardware related IOCs are the DHH IOC and the PowerSupply IOC. The DHH IOC provides all PVs that are needed to configure the DHH itself but also all of the ASICSs on the half-ladders. 


\subsection{ASIC Configuration}

While EPICS provides the high-level access to hardware components, the direct interaction protocols are implemented in the IOCs in form of drivers. The most crucial IOC is the DHH IOC, which is responsible for translating EPICS commands to hardware interactions on the DHE FPGA and also the ASICS on the PXD half-ladders.

Communication between the controlling computer and the DHE units is realised over Ethernet using the UDP-based IPBus protocol [71]. The ASICs on the half-ladders are organised in one JTAG chain and ordered as depicted in fig. 5.3. On the DHE a hardware JTAG master is implemented to control the ASICs. The DHE IOC implements a software JTAG master in form of an EPICS driver, which translates high level EPICS operations to JTAG commands, which are transmitted to the hardware master via the aforementioned IPBus protocol and sent to the ASICs in the chain.

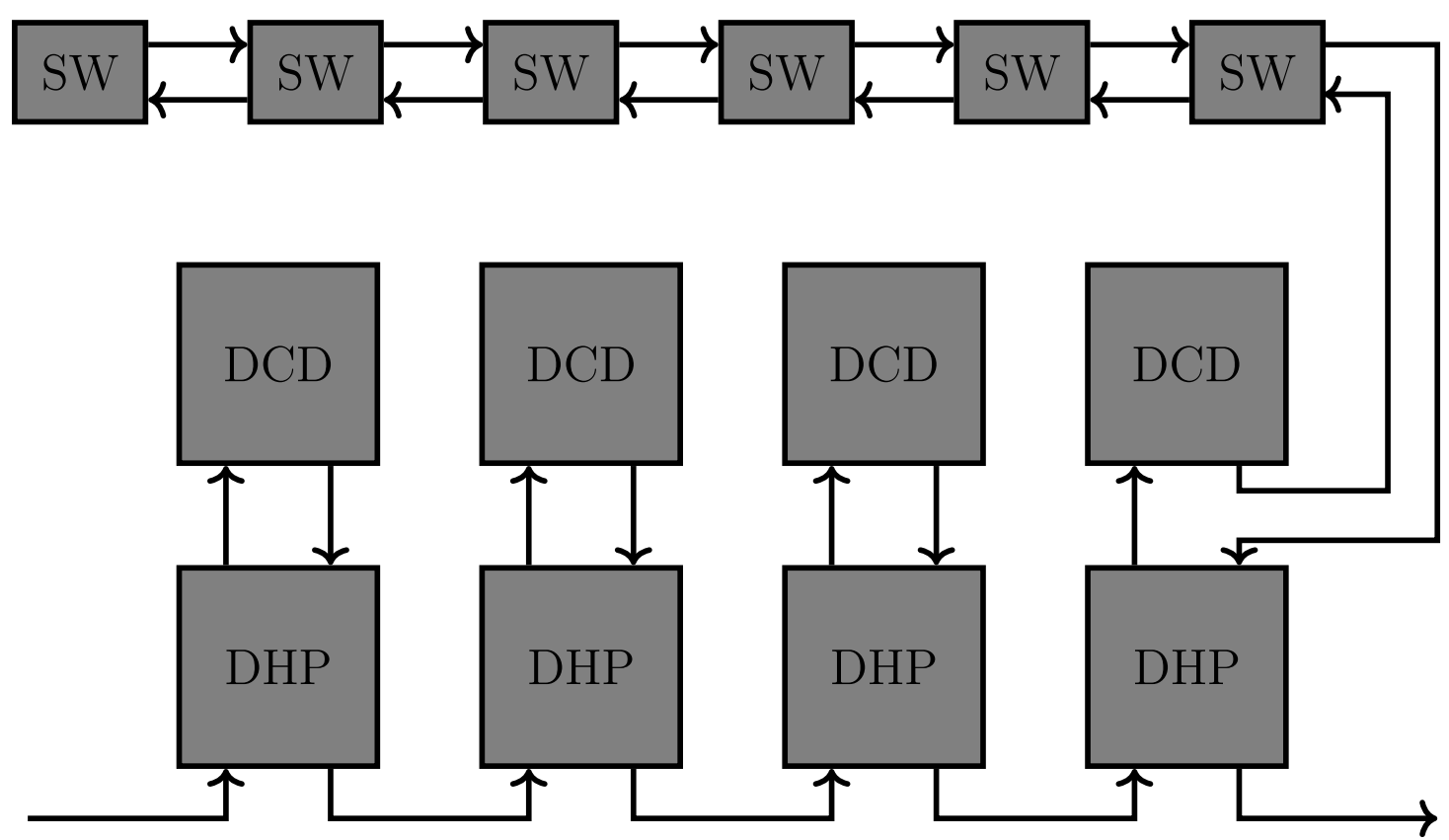

Figure 5.3.: Visualisation of the JTAG chain of a PXD half-ladder. The DHPs can be configured to exclude their attached DCD to "shorten" the JTAG chain.

\subsection{Configuration Database}

As described before, the IOCs allow to interact with all hardware components of the PXD, including the ASICs. The current status/configuration of the system is defined by the values of all these PVs. These sets of values are stored as commits within an SQL based database. For the laboratory setups this database was hosted in Heidelberg. For Belle II it is hosted directly at KEK. The interaction to the database is done via an 
IOC, the ConfigDB IOC. This soft IOC provides a commit-ID PV which can be set to load the corresponding configuration from the database.

For each PV defined in the loaded commit a configuration PV with the same name but different prefix is created which can be accessed by other IOCs. The commits can contain the configuration of a single half-ladder or the full PXD system. The configuration of the system itself is done via Sequencer IOCs that copy the values from the configuration PVs to the system PVs. This interplay of the various IOCs and databases is visualised in fig. 5.4.

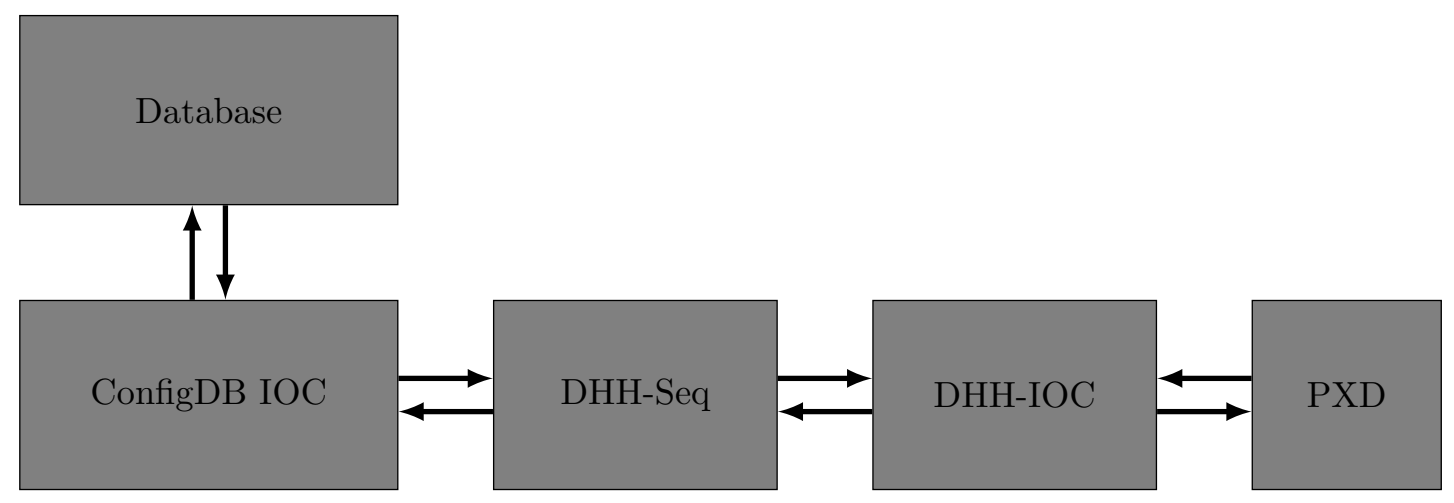

Figure 5.4.: Visualization of the IOC interplay and communication needed for the configuration of the PXD. Once triggered the ConfigDB IOC loads a commit from the (remote) database and creates PVs that can be accessed by other IOCs in the network. The sequencer IOCs steer the order in which the values from the database PVs are copied to the hardware PVs.

\subsection{PXD Startup Sequence}

The previous section mentioned a special kind of EPICS IOC, a sequencer. A sequencer can be its own IOC or it can be part of an existing IOC. It works as a state machine and is used for the control of the PXD DHH and power supply system. The sequencers used in the PXD project are based on the State Notation Language and Sequencer [72] (SNL) project.

SNL is a free software and fully integrated into EPICS. The software allows to define one (or more) state machines with an arbitrary number of states and state transitions. As it is fully integrated into the EPICS system, it can read from and write to PVs created by other IOCs and use the values of the PVs to initiate a state transition. Besides reading and writing to $\mathrm{PVs}$ it also possible to execute $\mathrm{C}$ code which gives the possibility to have more complex operations and calculations within the states.

The PXD slow control system has three major sequencers, the DHH Sequence, the PS Sequence and the PS Control sequence. The PS Sequence is responsible for the correct powering of the half-ladders. It applies the voltages in the correct order and with the 


\section{Slow Control and Calibration Software}

right speed. Using a sequencer for the powering is beneficial as there are over 20 voltages that have to be set in the correct order. The powering is further complicated by the fact that between the ramping of the voltages the ASICs have to be configured at several steps. This is done by the DHH sequencer which has to communicate with the PS Sequence via handshakes to make sure that the power-up/down is performed correctly. Any deviation from the pre-defined sequence could damage the attached half-ladder severely. The last sequencer, the PS Control sequence is a light-weighted sequencer that is used to control up to 40 half-ladders simultaneously. The user can select the desired half-ladders and ramp them in parallel to a common state. The hierarchy and relation-

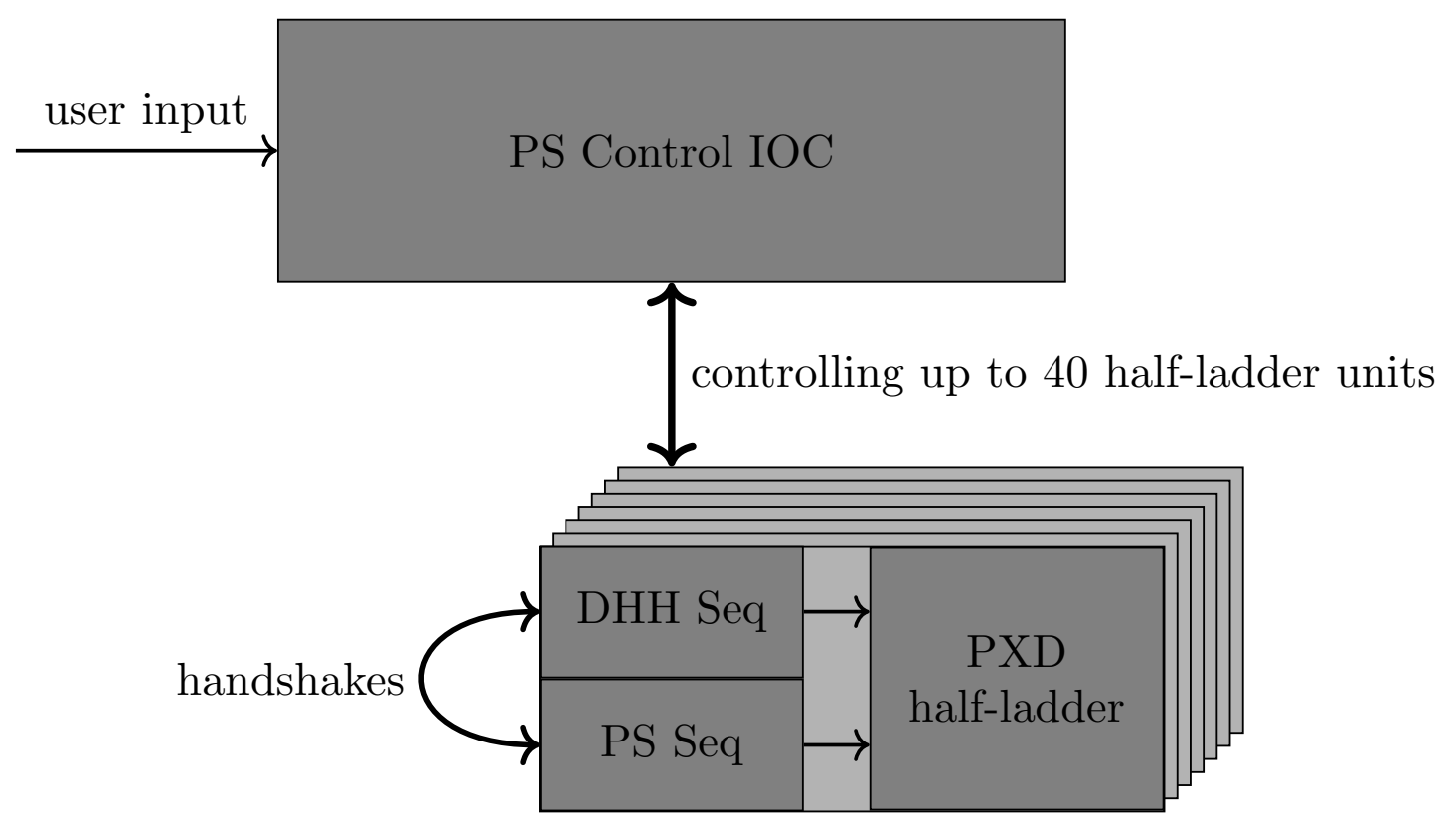

Figure 5.5.: Hierarchy of the Sequencer IOCs. A global PS Control IOC controls up to 40 half-ladder units, where each unit has its own DHH and PS Sequence IOC. The local sequencer communicate via handshakes with each other and control their half-ladder (via the DHH and Powersupply IOC).

ship of all three sequencers can be seen in fig. 5.5.

The DHH sequence is the centre of the whole EPICS sequencer slow control system. It communicates with the DHH, configures the ASICs, steers the PS Sequence and maps the state of an individual half-ladder to one of the general Belle II slow control states: OFF, STANDBY and PEAK. In addition, there are two error states, TRIP and ERROR. The former is not used by the PXD slow control and all severe errors of the system lead to the ERROR state. In the framework of this thesis, the existing DHH sequence was extended with additional features to fulfil all requirements for the laboratories but also for the operation at Belle II.

Figure 5.6 shows how the slow control states are ordered and how the transitions are 

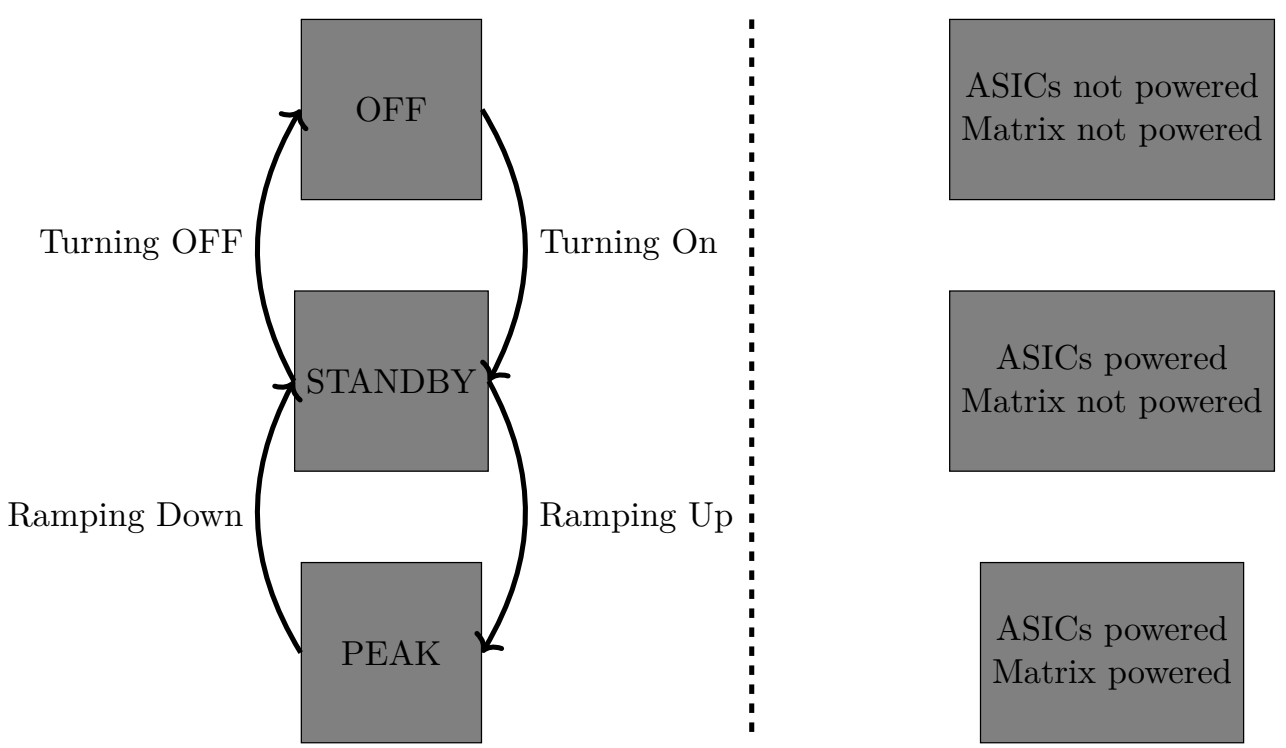

Figure 5.6.: States of the Belle II global slow/run control (left) and their meaning for the PXD slow control system (right).

named. It also shows the corresponding status of the PXD system. In the OFF state everything is unpowered, in the STANDBY state the ASICs are powered and configured and in the PEAK state the DEPFETs are powered as well, which means that the system is ready to take data. The PS Control Sequence calculates a common PXD state based on all individual half-ladders and forwards it to the global Belle II run control.

\subsubsection{Pedestal and 2-Bit DAC Offset Correction Upload}

At the start of this thesis work the DHH sequence was already in a state which allowed to power a half-ladder and configure it up to the PEAK state. There were, however, some features missing that are needed for the operation at KEK. One of the missing features was the upload of pedestal data. As described in section 4.5.4, the DHP needs the pedestals of the sensor for the hit-finding. These pedestals are usually constant over large times (at least several hours, often days), which is why they are stored in the configuration database. During a power-up they are retrieved from the database and uploaded to the DHP. In this thesis work additional states within the DHH sequence that take care of this upload were implemented. Figure 5.7 shows a flow chart of these states and describes their individual function.

The upload to the DHP itself is handled by the DHH IOC, which provides a PV that represents the corresponding memory of the DHP as a 262144 byte array. The mapping of the data in the configuration database is different from the one in the DHP which requires a remapping of the data before it can be written to the PV and then to the DHP. Listing 5.1 shows the function that is used by the DHH sequence to do this remapping. The data could now be written directly to the DHP memory. As pointed out earlier, 


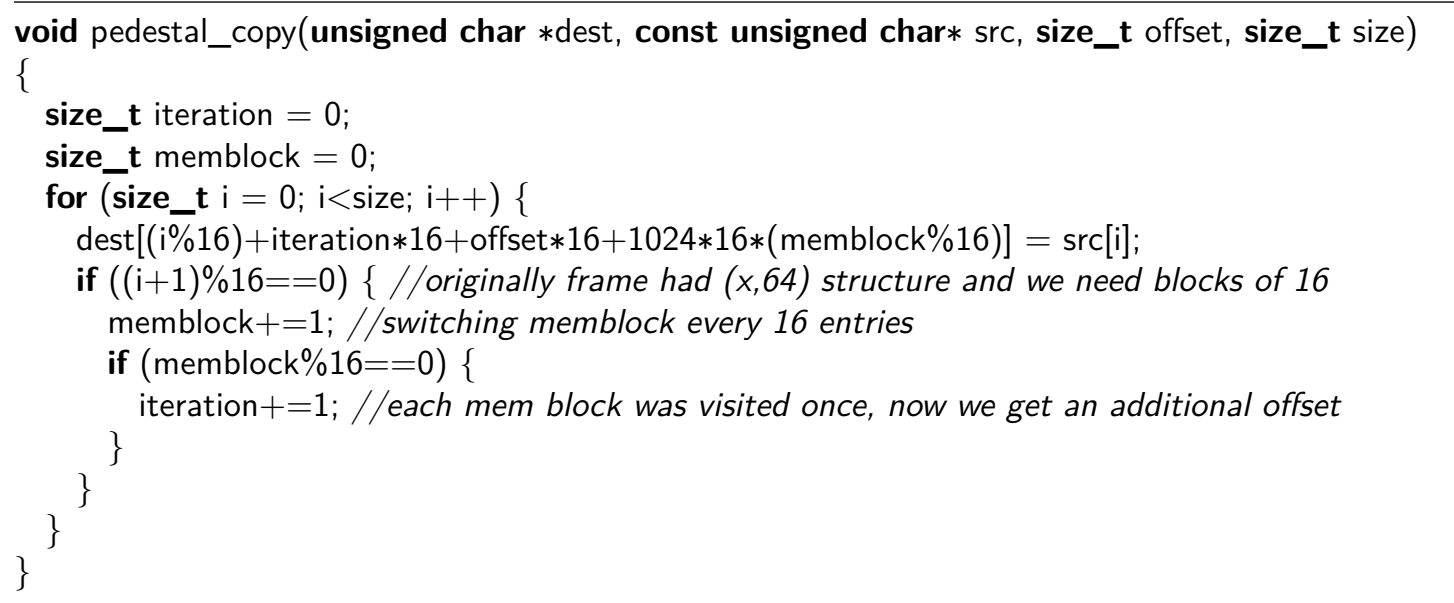

Listing 5.1: Function that maps data from the configuration database (src) to the DHP memory layout (dest). The for loop iterates over all 262144 bytes. After 8 bytes ( 1 word in the DHP mempory), the memory block is switched. Once all memory blocks have been visited once, data is written to the first block again but with an additional offset (the second "row" of the memory blocks).

the memory of the DHP is larger than the actual pedestal data and a simple upload would produce unnecessary write operations to the memory. To avoid this the upload procedure splits the dataset and uploads it sequentially. For this sequential upload, the start address to which the data is written on the DHP is changed after each upload. After completion an optional check of the uploaded data can be performed. Due to limitations of the DHH firmware this check is rather slow and a complete check of the memory takes several minutes. If the check is performed and an error is found in the uploaded data, the procedure is repeated.

The second addition to the DHH sequence was the 2-Bit DAC offset correction. Once an optimal set of values is found for this correction, it has to be stored within the DHP memory like the pedestal data. The implementation of this upload procedure is therefore very similar. The main difference is the mapping of the data. As the dataset is smaller than the pedestal data, the check of the uploaded data is also faster, but still around a minute, which is why this check is optional as well.

The third big feature added was the activation of the analog common mode correction (ACMC) of the DCD. While the configuration of the ASICs was part of the sequence before, the configuration of the ACMC requires special actions that were not covered by the "normal" ASIC configuration step. In this configuration steps PVs are set and the registers on the chips are written to. The configuration of the ACMC requires multiple steps of PV set actions. Therefore a separate state was created during which the necessary steps are performed. 


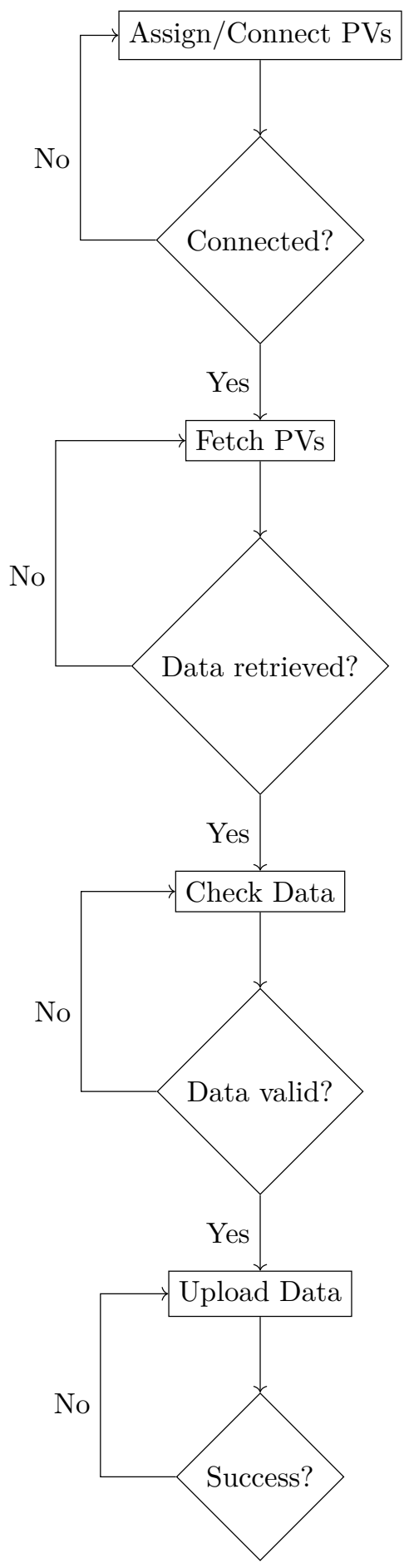

Connection to configuration database PVs is established.

The pedestal data is copied from the PVs of the configuration database IOC to local variables of the DHH sequence.

Sanity checks on the retrieved pedestal data are performed, e.g. right size, not empty, etc.

Data is mapped and uploaded to the DHP (via the DHH IOC).

Figure 5.7.: Steps for the pedestal upload. 


\section{Slow Control and Calibration Software}

\subsection{Calibration Software Framework}

The previous sections covered the Slow Control and the usage of EPICS. This section gives more information on the Calibration Software Framework which was developed over the years and used for the laboratory measurements of the PXD. The framework is written in python and consists of several libraries and calibration scripts. To interact with the hardware of the sensor the PyEpics [73] library is used. This library allows to read and write to EPICS PVs from any python script. The actual hardware interaction is then executed by the EPICS IOCs. In 2017 guidelines for the calibrations script were formulated in order to further structure the framework. Each calibration package is split into three parts.

Measurement: The measurement script configures the system (ASICs on the half-ladder as well as the DHH backend) and records the necessary data. After the measurement the initial configuration is restored. Parameters of the measurement are configured via a configuration file.

Analysis: The analysis of the recorded files is the secondary step of the calibration. Together with the metadata that is stored by the measurement script, it is possible to perform the analysis at any time after the measurement on any computer. Similar to the measurement script, parameters are specified in a configuration file. This script does not interact with the hardware at any point. Results of the analysis, usually optimised ASIC settings, are saved after the analysis in a standardised format that allows an easy upload to the system.

Update: The last (optional) step is the upload of the results of the analysis script. The upload script can either update the system or create a new commit in the configuration database or do both.

While this approach gives a clear structure to the calibration scripts, it makes them less flexible. The decoupling of the measurement and the analysis process requires to scan the whole parameter phase space and find the optimum later where a direct analysis after each measurement step might offer a faster way to the optimum. As most calibrations are done on a time-scale of minutes, this is not a severe issue.

Highspeed Links: Test of the highspeed links that connect the DHPs to the DHE. Three parameters have to be optimised to ensure a stable communication. A detailed description and discussion of the DHP high speed data links and their optimisation can be found elsewhere [74].

DCD-DHP communication: The communication between the DHP and the DCD is tested by sending a fixed testpattern from the DCD to the DHP. The sampling point on the DHP can be steered with delay elements which have to be configured correctly, see also section 4.5.4. 
2 Bit DAC offset correction: The optimization of the 2 Bit DAC offsets requires the upload of several testpatterns to find the optimal IPDAC value and the optimal setting for each pixel.

ADC Transfer Curves: Optimal parameters for the DCD are found by recording ADC transfer curves. There are two voltages and three important settings on the DCD that influence its performance. Because of the large phase space this calibration requires a long time and is only doable when the phase space is sliced by performing multiple two dimensional scans.

DEPFET voltages: To find the optimal DEPFET voltages is a non-trivial task. The gate, clear, drift and depletion voltage create a complex potential landscape within the DEPFET cells. The script is used to record data from radioactive sources and vary those voltages to optimise the charge collection and the amplification of the DEPFETs.

\subsection{Utility IOC}

During a regular start up of a PXD half-ladder, the configuration of the ASICs is handled by the DHH Sequence as described above. Once the system is fully powered, no large changes of its configuration are foreseen while data is being recorded. However, in the laboratory, it is often necessary to reconfigure individual ASICs for specific tests. As it is inconvenient to restart the system for each reconfiguration, a python based EPICS IOC was written to handle these reconfigurations, the Utility IOC. The creation of this IOC was part of this thesis work. This IOC uses the PCASPy [75] library, which allows to create EPICS IOCs with a high abstraction level.

The usage of EPICS allows to easily access the configuration database to get the necessary information for the configuration of the ASICs. For each half-ladder an instance of the IOC is started. Control of the IOC is done through EPICS control PVs, which can be set to trigger an action of the IOC. For each triggered action a new thread is spawned by the IOC. As long as the thread is active, the main process blocks further configuration requests by the user.

Prior to the Utility IOC, a functional similar IOC was used with the disadvantage that it was not easily scalable to more than half-ladder, which is why it was replaced. The IOC is designed so that is more flexible than the DHH Sequence, allowing to configure for example only one DHP or all DCDs but no other ASIC. Upload of new switcher sequences, pedestal data or 2-Bit offset correction data is also supported by the IOC. The data for these uploads can be retrieved from an arbitrary commit in the configuration database.

Another important feature of the Utility IOC is an automatic temperature measurement of the half-ladder using the DHP's temperature sensor. To prevent damage to 


\section{Slow Control and Calibration Software}

the half-ladder due to overheating, the temperature is read out in a definable time interval and compared to a given threshold value. If three consecutive readings of the temperature are above the critical value, an emergency shut-down of the power supply is triggered.

\subsection{Calibration IOC}

When operating a single device (PXD half-ladder or Hybrid5) the execution of calibrations is a simple task due to the structure of the calibration software framework. This does become more difficult when a large number of modules is meant to be calibrated in parallel. As the parallel operation and calibration of 40 half-ladders by non-experts is the target of the PXD project, a solution had to be developed. There were several requirements for this software solution:

1. The control of up to 40 half-ladders, which share (partly) their data-readout system via a common DHC.

2. Easy to use for non-experts at KEK with safeguards preventing faulty operation and damage to the PXD.

3. Giving feedback of the current calibration status.

4. Being expandable for different kind of calibrations.

Based on these requirements it was decided to build a dedicated EPICS IOC, called Calibration IOC. Like the Utility IOC, this IOC uses the PCASPy library. As the software calibration framework is written in python, an easy integration into the existing framework was also possible.

The IOC can interact through EPICS with other IOCs and can itself be controlled by PVs it creates on its own. Through these PVs actions of the IOC can be triggered, e.g. start of a certain calibration. Giving feedback to the operator is also possible through dedicated status PVs. Like for any other EPICS IOC, a GUI using CSS can be created. Figure 5.8 shows the GUI of the Calibration IOC used during phase 2. Through the GUI individual half-ladders can be selected by the user and a calibration can be started. Through a progress bar the user is informed about the current state of the calibration. If a calibration of device $X$ is started, the corresponding DHC, through which the data of the device has to go and which ultimately triggers the data taking, is blocked. While this calibration is ongoing, the IOC makes sure no additional calibration on one of the other devices connected to the same DHC can be started.

Internally, a new process is spawned whenever a new calibration is started. By doing so, the main process remains responsive to commands from the user and is not blocked by the calibration. In order to avoid code duplications, the Calibration IOC does not implement the calibrations by itself but uses the implementations already present in the 


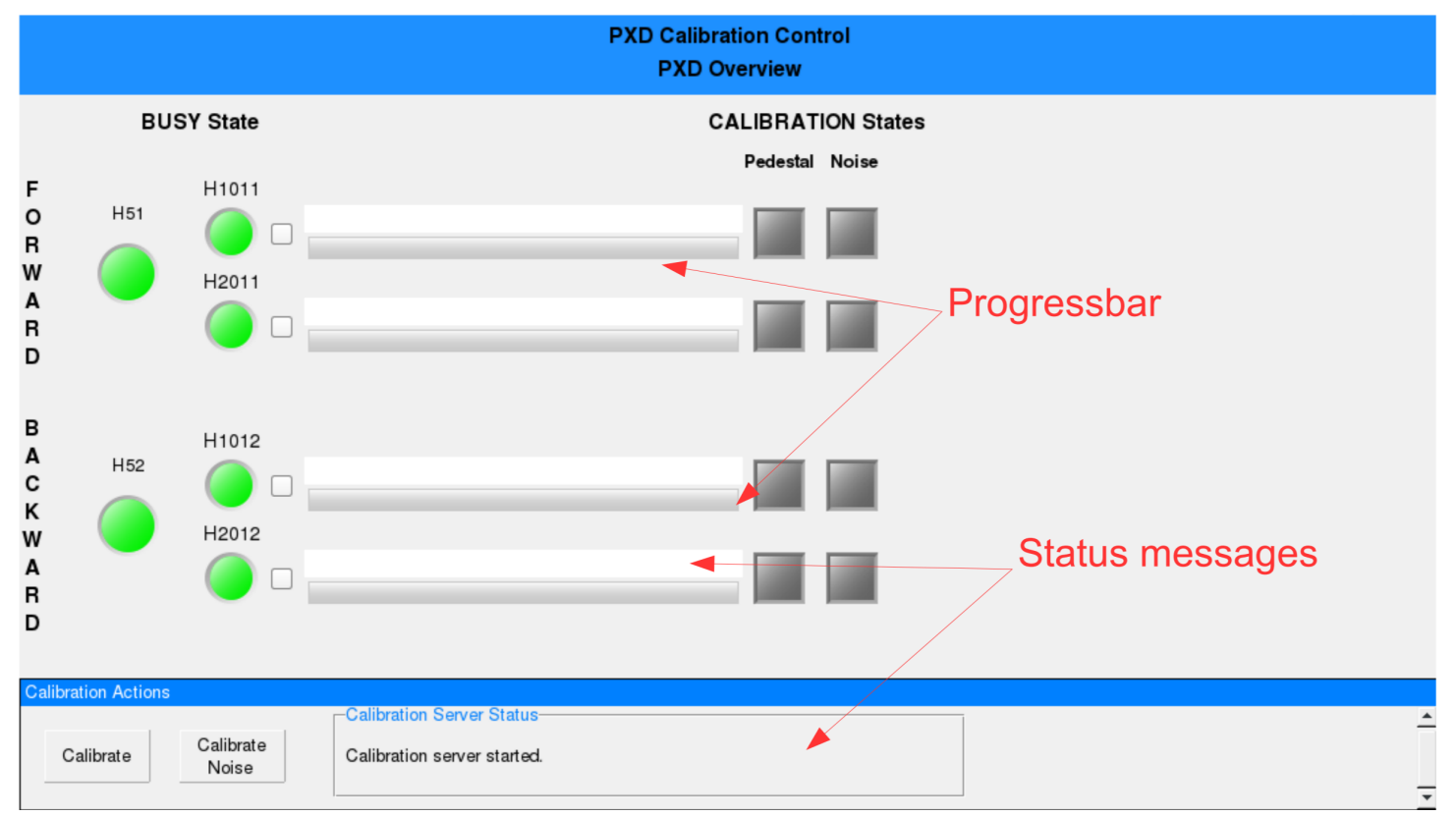

Figure 5.8.: Graphical user interface of the Calibration IOC during the phase 2 operation with four half-ladders and two DHCs. For each half-ladder/DHE and DHC, a LED shows the busy state of the device. Progress bars and text fields inform the operator about the current state of a configuration.

calibration software framework. This means that the same measurement-analysis-update strategy is used. To work with the Calibration IOC a standardised interface had to be defined, to make the function callable and give proper feedback to the IOC.

The most common calibration is the pedestal taking. Due to changes of the operation point, changes of environmental conditions or radiation damage to the sensor it is often necessary to update the pedestal values stored in the DHP memory. That is why the pedestal calibration was the first calibration implemented in the IOC. To have a quick look at the results of this particular calibration type, the IOC provides special PVs to hold the information. Using another CSS widget, see fig. 5.9, the quality of the calibration can be checked easily by the operator. This so-called pedestal monitor show the uploaded pedestal values as a heatmap and provides additional information about the pedestal value distribution like average value and standard deviation.

The Calibration IOC is configured with a configuration file that describes the system that has to be controlled. In particular, the numbers of DHCs and DHEs, their relation to each other and their names have to be known to the IOC. Based on this configuration, the IOC creates and provides the appropriate status and control PVs. When a calibration function is called (e.g. pedestal calibration), the information about the setup topology is passed along to the function so that correct data-taking and analysis is trig- 


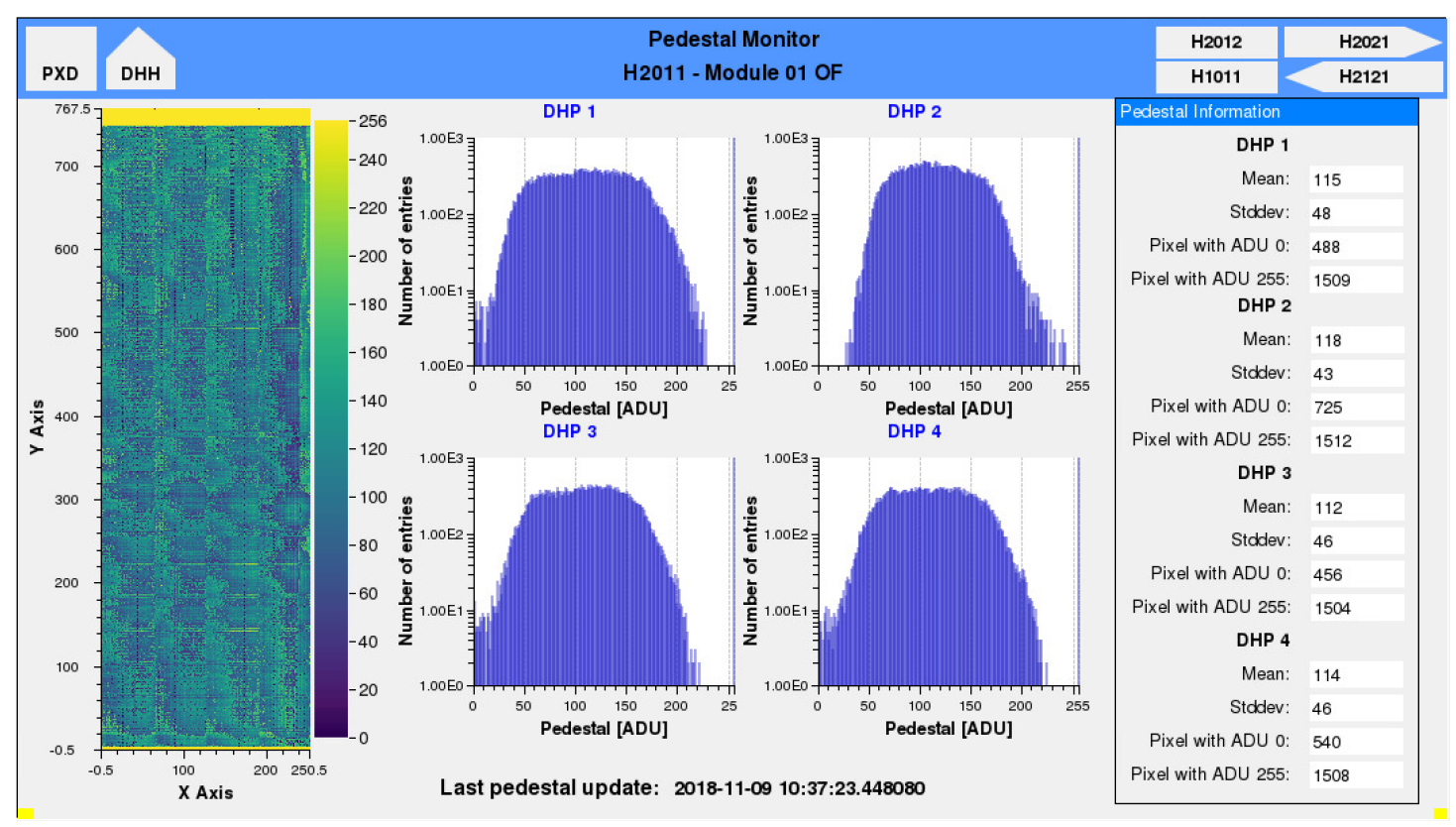

Figure 5.9.: Screenshot of the so-called Pedestal Monitor widget. The widget shows a 2D presentation of the uploaded pedestals and gives more detailed information about the distribution of the pedestals for each ASIC pair.

gered. To be compatible with laboratory type setups, the IOC also supports a special mode of operation in which a dummy DHC object is created. Using this approach the remaining logic of the IOC does not have to be changed for this case. This does, of course, mean that the implementation has to be done inside the calibration functions.

\subsection{ELOG}

Documentation of measurements and analyses is an important task during calibrations in the laboratory, but also during large-scaled test campaigns or the operation of the Belle II experiment. There is a variety of tools and software packages available to log information electronically. The tool used by the PXD collaboration and also for the Belle II experiment is the ELOG [76].

The ELOG software package allows to create servers that are easily accessible over a web interface and can be used to list and document measurements. It also allows to filter and sort entries or to search for specific ones. There is also the possibility to upload images and other files as attachments to the entries. These web logs can be configured in many aspects to fulfil the users specific needs. In addition, the read and write access can be limited to certain users or user groups. 


\subsubsection{ELOG Usage in the Laboratory}

While the ELOG provides an easy way to log operations, it still requires manual intervention by the user. This procedure is prone to errors, as the user might unintentionally enter wrong information. For the mass testing of the PXD half-ladders for Belle II, a large group of users had to log their progress in a common logbook. To compare the entries (of the same measurement type) it was therefore also desirable to use the same structure in the logbook entries. That is why an automation of the logging procedure was developed during this thesis work.

A python library was written, which contains the necessary functions to interact with an arbitrary remote logbook. The integration to the measurement and analysis scripts is done using an object oriented approach, where the scripts create an elog object with an identifier that specifies which kind of measurement or analysis it is. The specifics of the elog entry (e.g. message text and attachments) is defined in the elog library and has therefore to be prepared beforehand. Figure 5.10 shows the web-interface of the logbook that was used for the PXD mass testing and 5.11 shows how a pedestal analysis entry looks like in the logbook. The individual entries contain all relevant information of the measurement/analysis and also provide plots as well as the most important results. The "measurement entries" also contain a file with the values of all EPICS PVs at the start of the measurement, making it easier to check the actual configuration of the system which might in some cases differ from the configuration that is defined in the commit that was loaded at the time of the measurement.

The automation of the logbook entries proved to be very helpful during the PXD mass testing and made it easy to quickly exchange information between the different laboratories. The entries also made it easy to create meta logbook entries summarising the analysis results of one half-ladder or on a second level all half-ladders. 
Figure 5.10.: Screenshot of the PXD-Mass-Testing logbook. A filter is applied to only show entries belonging to a specific half-ladder (W43_IB). Through the columns a quick overview of the measurement and half-ladder type is possible. The text field is used to give more detailed information about the measurement or analysis. 


\begin{tabular}{l|l}
\hline Message ID: 1081 & Entry time: 2017/11/19 Sun 14:15 UTC \\
\hline Author: & goe-desktop04 \\
\hline Category: & Analysis \\
\hline Type: & Pedestal Analysis \\
\hline Device: & pxd9 \\
\hline Module: & W43_IB \\
\hline Moduletype: & ib \\
\hline CommitID: & 768 \\
\hline
\end{tabular}

Pedestal Analysis started at Sun, 19 Nov 2017 14:14:25.

Analysis of 1000 frames of pedestal data:

For the analysis/plotting pxd9 mapping was used.

The unconnected channels were masked.

No noise masking was used.

The offline common mode correction was not used.

The analog common mode correction on DCD 1 was Off

The analog common mode correction on DCD 2 was Off

The analog common mode correction on DCD 3 was Off

The analog common mode correction on DCD 4 was Off

The offset correction on DHP 1 was On

The offset correction on DHP 2 was On

The offset correction on DHP 3 was On

The offset correction on DHP 4 was On

Detailed information per DCD:

DCD 1:

0 channels showed an ADU value of 0,0 an ADU value of 255

The total spread is 238.0 , the median is 134.0 with a standard deviation of 30.1286804244

DCD 2:

0 channels showed an ADU value of 0,0 an ADU value of 255 .

The total spread is 187.0 , the median is 137.0 with a standard deviation of 23.5747777282

DCD 3:

0 channels showed an ADU value of 0,0 an ADU value of 255 .

The total spread is 183.0 , the median is 149.0 with a standard deviation of 24.3467117242

DCD 4:

0 channels showed an ADU value of 0,0 an ADU value of 255 .

The total spread is 164.0 , the median is 148.0 with a standard deviation of 22.9970072624

Data is stored on goe-desktop04.ph2.physik.lokal in /data/ext04/W43_IB/pedestal_scan/2017_11_19_002.

Pedestal Analysis stopped at Sun, 19 Nov 2017 14:15:11.

Total time passed: 0:00:46

\begin{tabular}{l|l}
\hline Attachment 1: & noise_plot.pdf 462 kB | show | show a11 \\
\hline Attachment 2: & pedestals_plot.pdf 470 kB | show | show a11 \\
\hline Attachment 3: & pedestals_mult_plot.pdf $475 \mathrm{kB} \mathrm{|} \mathrm{show} \mathrm{|} \mathrm{show} \mathrm{a11}$ \\
\hline Attachment 4: & results.npy 4.638 MB \\
\hline Attachment 5: & analysis.npy 1.501 MB \\
\hline
\end{tabular}

Figure 5.11.: Example of a pedestal analysis logbook entry. In the text the most important settings of the DCD and DHP ASICs are listed together with a short summary of the results. Attached to the entry are the created figures and binary data files with the results of the analysis. 


\section{Slow Control and Calibration Software}

\subsubsection{Automation of Belle II Run Logbook Entries}

As mentioned above, the ELOG software is also used for the logging of runs at Belle II. The same reasons for an automation of the logging procedure as in the laboratory are true there as well. This is why another PCASPy IOC was written which has the task to create elog entries at the end of each run and transmit them to the official Belle II run logbook. This IOC is called elog messenger.

Most of the information that is written to the logbook entries comes from the slow control network of Belle II and the subsystem. While the PXD and the SVD slow control use EPICS, the Belle II run control uses NSM2 [77] (Network Shared Memory II), which is the successor of the NSM system that was used for Belle. The NSM2 system is accessible via EPICS through a dedicated gateway so that access to variables in the NSM2 network is possible over EPICS PVs.

The PVs provided by the global Belle II run control are used by the IOC to check for a start/end of run. As soon as a run stops, the IOC checks various status PVs like beam currents, magnetic field strength of the solenoid and various others. In addition, a request from the IOC to the Belle II EPICS archiver is sent to get information about the recorded luminosity and number of triggers. The logbook entry is then submitted to the remote elog. Once the entry is submitted and listed there, the Belle II shifters can edit the entry to add any additional information. Attached to the logbook entries is also an XML file, which lists the values of various PVs of the Belle II subsystems and the SuperKEKB accelerator in a machine readable format. Figure 5.12 shows an example of an logbook entry belonging to a Luminosity run where all sub-detectors were included in the data taking.

In addition to the IOC used for the global Belle II runs, there is a fork of the IOC that records and submits entries for the PXD logbook. Internally this second IOC works very similar to the elog messenger. It does, however, have additional PXD features. The main addition is the control of the local PXD DAQ. As described in section 4.6.3, data from the DHH system is not only sent to the global Belle II DAQ but also (partly) to the local PXD DAQ. On the computer that receives this data stream the local DAQ software has to be started and stopped at the begin and end of run, which is controlled by the PXD elog IOC. Attached to the entries are also summary figures, showing the SuperKEKB beam currents and the trigger rates of the half-ladders over the course of the logged run. Additionally, hitmaps and cluster charge histograms, filled with information from the Local DAQ Online Monitor are plotted. An example of such a summary figure can be seen in fig. 5.13. 


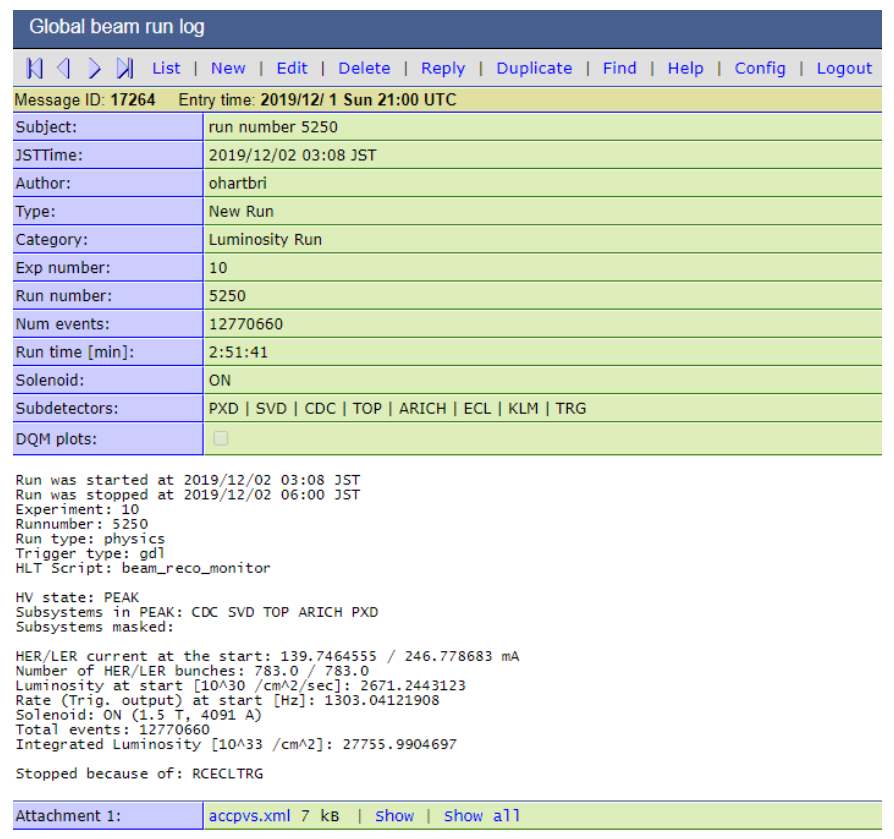

Figure 5.12.: Example of a Belle II global run logbook entry. The start time of the run is listed in conjunction with its duration and end point. The entry also contains information about the recorded luminosity and which subsystems of Belle II were active during the run. An xml file containing information about accelerator conditions is attached as well.

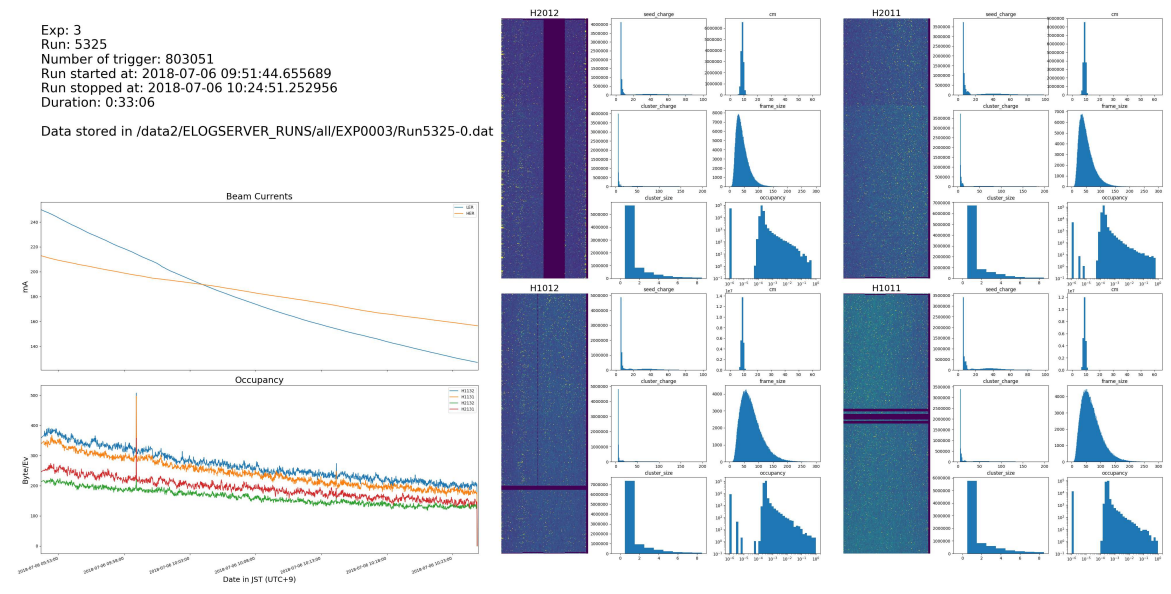

Figure 5.13.: Example of a PXD summary figure of a run logbook entry from phase 2 . The figure shows the hitmaps of the four phase 2 sensors together with various histograms giving information like cluster charge, common mode distribution and data frame size. 



\section{CHAPTER 6}

\section{Phase 2 Commissioning}

The commissioning and operation of the SuperKEKB accelerator and the Belle II experiment was subdivided in three phases. In the first phase of the commissioning the SuperKEKB accelerator was operated without its final focusing magnets and Belle II was not inserted. Therefore, no collisions of particles occurred. The main emphasis of this phase was on accelerator test and vacuum scrubbing of the beam pipe as preparation for the subsequent phases. During phase 2, which is the main focus of this chapter, the focusing magnets were in place and Belle II, without the complete VXD, was placed at the interaction point. The VXD that was installed during phase 2 consisted of only four PXD half-ladders (arranged in two layers) and four SVD ladders building layers four to six. The operation with the full VXD installed is referred to as phase 3 .

\subsection{BEAST II}

As the VXD was incomplete during phase 2, the free space was filled by additional sensors. These sensors had the task to measure the background rates. This VXD configuration was named the BEAST II experiment (Beam Exorcisms for A Stable Experiment). In the following these additional subsystems and their characteristics will be introduced.

FANGS: The FANGS (FE-I4 ATLAS Near Gamma Sensors) system uses ATLAS IBL [78] pixel detector modules arranged on three ladders. Each ladder has five of these modules consisting of a FE-I4 [79] readout chip and a $200 \mu \mathrm{m}$ thick n-in-n planar silicon sensor. Because the FE-I4 was designed to withstand the harsh radiation environment at the LHC (Large Hadron Collider) [3], it was chosen to study the backgrounds during phase 2. More details about the FANGS system can be found in $[80]$. 


\section{Phase 2 Commissioning}

CLAWS: The CLAWS (SCintillation Light And Waveform Sensors) system is a scintillation based sensor and is descibed in detail in [81]. Like FANGS the system uses a ladder design with eight sensors per ladder. In total two CLAWS ladders were installed in the VXD volume. The readout of the sensors is realised by a digital oscilloscope, which allows a high time resolution. Because of this high resolution, CLAWS main purpose was the study of injection based backgrounds during phase 2 .

PLUME: The PLUME (Pixelated Ladder using Ultra-light Material Embedding) system [82] was designed for a future linear collider and used during BEAST II to measure background rates. PLUME is an air cooled system that uses a doublesided ladder of MIMOSA-26 sensors. Each ladder has 2x6 sensors with a $2 \mathrm{~mm}$ thick Si-carbide foam between them. Two ladders were installed in the VXD, one of them at a radius of $5 \mathrm{~cm}$, the other one tilted with a radius of $5 \mathrm{~cm}$ at the closest and $9 \mathrm{~cm}$ at the farthest point. More details about the PLUME system during phase 2 can be found in [83].

Figure 6.1 shows the phase 2 configuration of the VXD with the SVD, PXD, FANGS, CLAWS and PLUME sensors as a CAD drawing.

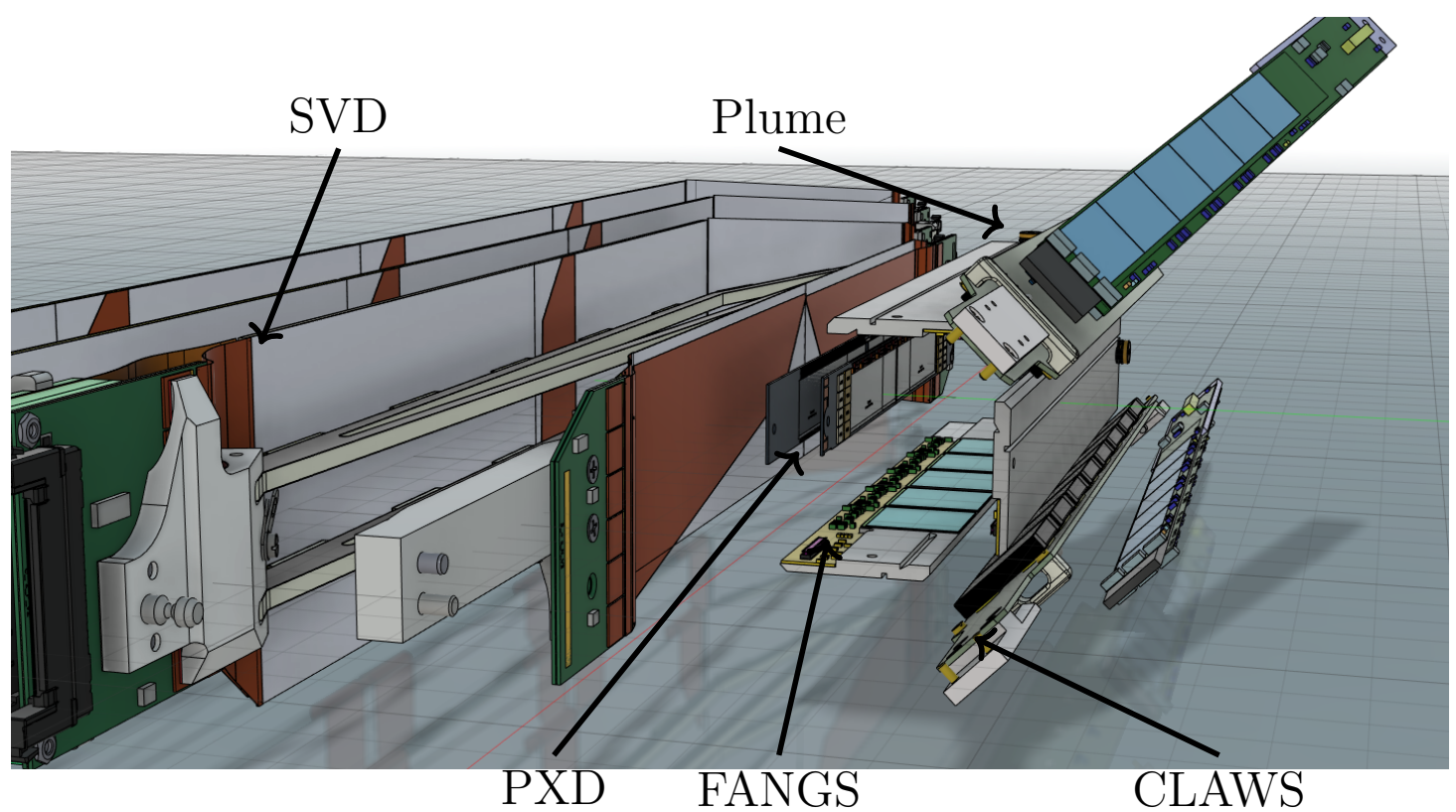

Figure 6.1.: CAD drawing of the VXD during phase 2. The support structures, housings and pipes are not shown. Adapted from [84].

In addition to these system that all have the capacity to measure background rates with a spatial resolution, there was a also a dedicated system to measure the ionising dose rate, the diamond system. The single-crystal sensors had a size of $4.5 \times 4.5 \times 0.5 \mathrm{~mm}^{3}$. 
Like for other semiconductor detectors, particles going through the sensitive volume create electron-hole pairs. Through electrodes an electric field is created which causes the charge carries to drift. The resulting current is proportional to the measured dose rate. For precise results a calibration of each sensor is required.

The diamond system had two tasks during phase 2. First of all, it was used for a continuous dose rate measurement and secondly it was used as a beam abort system. The data for the continuous measurement was read out and archived with a rate of $10 \mathrm{~Hz}$. To distribute the information and for the archiving, EPICS PVs for the individual diamond sensors were provided. The diamond system and its results during phase 2 are described in detail in [85].

In total there were eight diamond sensors positioned around the beam pipe of which only four were read out at the same time. Figure 6.2 shows a drawing of the beam pipe with the positions of the diamond sensors around it. Four sensors were positioned in the forward direction at $z=138 \mathrm{~mm}$ and four in the backward direction at $z=-100 \mathrm{~mm}$ (relative to the interaction point of the beams). The azimuthal angles (in the Belle II reference frame) of the sensors are $55^{\circ}, 125^{\circ}, 235^{\circ}, 305^{\circ}$ for both backward and forward.

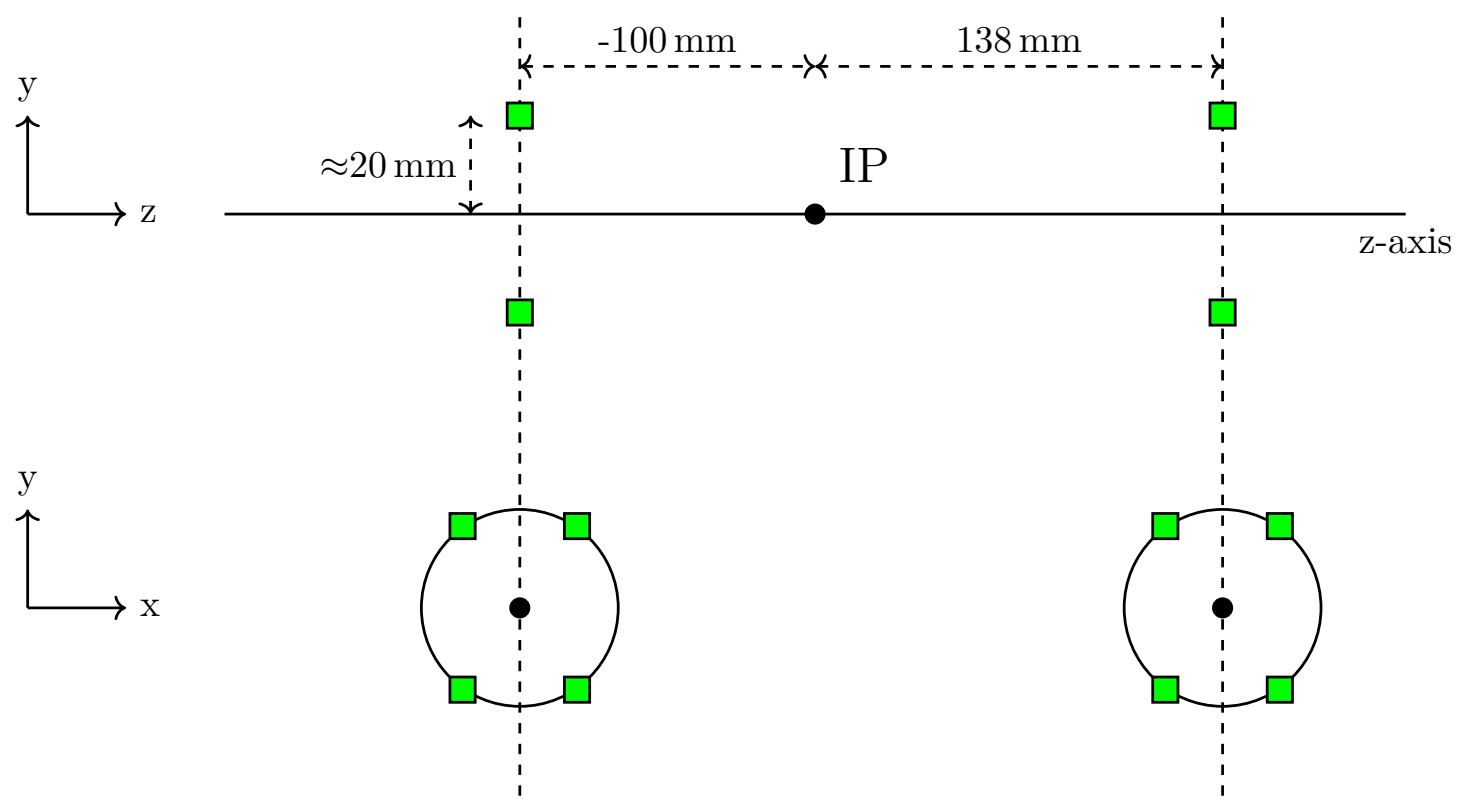

Figure 6.2: Sketch showing the positions of the diamond sensors (green boxes) relative to the interaction point (IP).

In addition to the diamond system, radiochromic foils were installed at several places in the VXD volume, including the positions of the diamond sensors. Three different types of foils were used, each of them sensitive to a specific dose range. Table 6.1 lists all types used during phase 2 together with their thickness and their dose range. The foils placed around the diamond sensors were $12 \times 18 \mathrm{~mm}^{2}$ pieces of HDV2 and EBT3. 


\section{Phase 2 Commissioning}

\begin{tabular}{lrrr}
\hline Type & Dose range $[k G y]$ & Thickness (total) $[\mu \mathrm{m}]$ & Thickness (active layer) $[\mu \mathrm{m}]$ \\
\hline B3 & $>2$ & 20 & 20 \\
HDV2 & $0.01-1$ & 110 & 12 \\
EBT3 & $0.00001-0.1$ & 280 & 28 \\
\hline
\end{tabular}

Table 6.1.: List of radiocromic foil types used during phase 2, from [86]. HDV2 and EBT3 have a substrate layer in addition to their active layers.

The same combination was used for the foils that were placed on the SVD ladders. On the FANGS and PLUME system all three types were used.

In contrast to the diamond system, the foils did not allow to monitor the radiation continuously as they could not be accessed once the VXD was installed in Belle 2. They were extracted after phase 2 had ended (August 2018) and then analysed. Depending on the level of radiation the foils change their color, which is used to determine the dose.

In addition to the systems described above, the BEAST II setup also featured pin diodes that were placed at the SuperKEKB focusing magnets to measure the radiation levels at this point. There were also MicroTPC units and He3 tubes to measure the fast and thermal neutron rate, respectively. Details about these systems can be found elsewhere [32].

\subsection{Pixel Vertex Detector Preparations in the Laboratory}

As mentioned above, only $1 / 10$ of the final PXD was installed for phase 2 . These four half-ladders, the W37_OF1,W41_IF, W46_IB, and W37_OB1 were characterised in the laboratory beforehand. Originally, it was planned to glue the half-ladders together to test the glueing process, too. Due to time constraints this plan was discarded. The four modules were distributed to German institutes with PXD laboratory setups. In Göttingen, the W37_OF1 half-ladder was characterised. Before the half-ladder was brought to Göttingen, basic sanity checks on a probe card station were performed in Munich. These tests included a power-up of the modules to check the current consumption of the ASICs and a configuration of the ASICs to test the JTAG functionality. These tests showed no abnormalities.

In Göttingen further tests and a calibration of the module were scheduled. For these measurements a dedicated test stand was set up, in which the half-ladder could be operated and characterised. Powering and readout of the half-ladder was realised with an ordinary laboratory set-up as described in section 4.6.3. Cooling of the half-ladder was realised by an aluminium cooling block which was connected to a water chiller. A close-up of the W37_OF1 half-ladder can be seen in fig. 6.3. As the mass testing procedures that were later used for the testing of the phase 3 modules were not yet defined, the testing of the W37_OF1 did not follow this procedure. The temperatures of the system were monitored continuously through the DHP temperature sensors. Table 6.2 


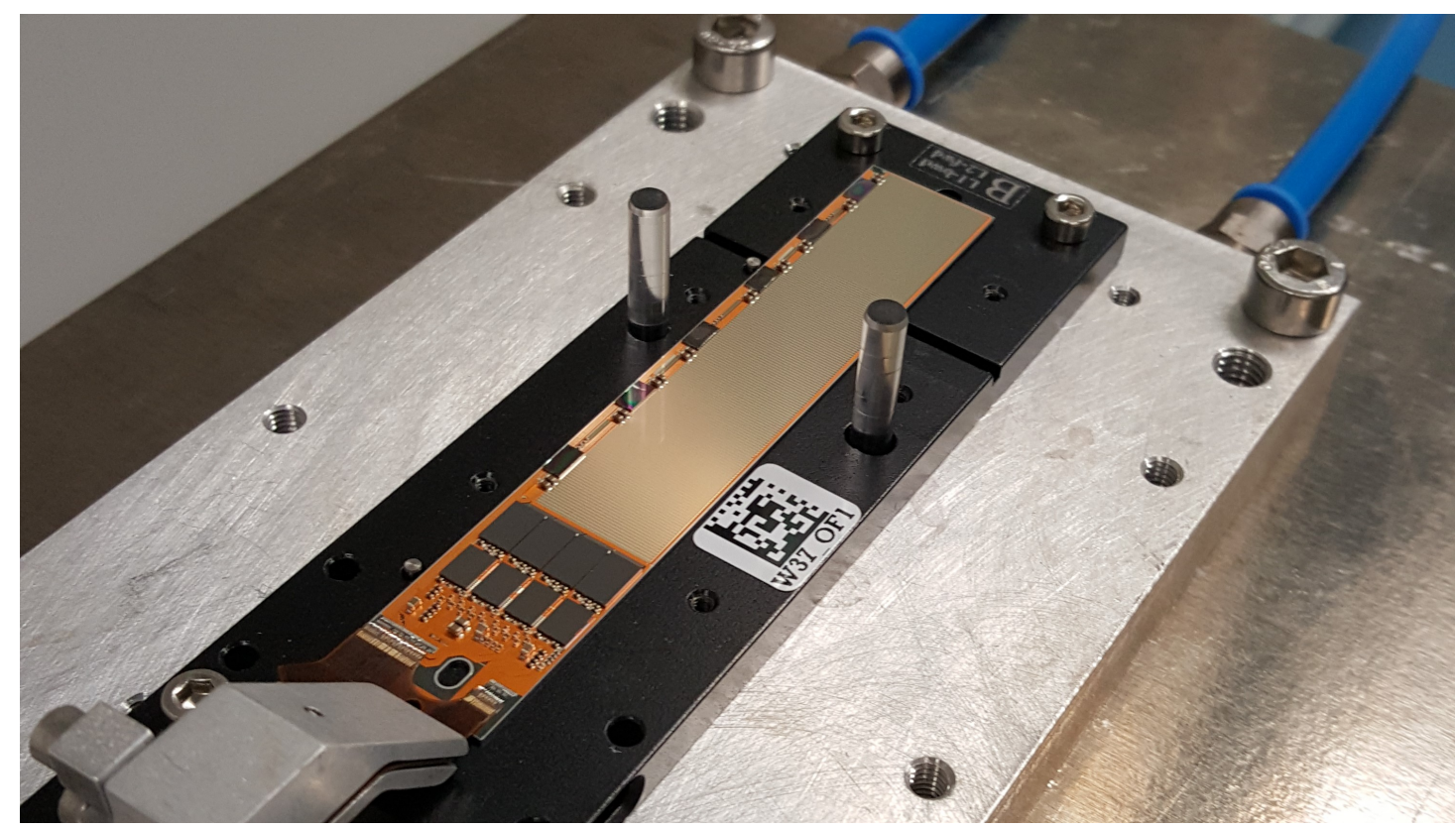

Figure 6.3.: Close up of the W37_OF1 half-ladder mounted on an aluminium cooling block. The cooling block is connected to a water chiller via two pipes (blue).

\begin{tabular}{l|r|r|r|r}
\hline & DHP & + DCD & + DCD $($ analog $)$ & + DEPFET Matrix \\
\hline Temperature $\left[{ }^{\circ} \mathrm{C}\right]$ & $25-30$ & $30-35$ & $50-58$ & $55-60$ \\
\hline
\end{tabular}

Table 6.2.: Temperatures of the W37_OF1 half-ladder measured by the DHP temperature sensors. The water chiller was set to $18^{\circ} \mathrm{C}$. The column names indicate which part of the half-ladder was switched on (cumulative).

summarises the temperatures of the half-ladder for different operation states. If the half-ladder was fully powered and ready for data taking, the temperature was $55-60^{\circ} \mathrm{C}$.

The first step of the calibration was the optimisation of the digital communication: DHP $\leftrightarrow$ DHE high speed data links and DHP $\leftrightarrow$ DCD data links. The optimisation of the latter was done using the DCD testpattern and a scan over the DHP delay element settings as described in section 4.5.4. For the former a scan over the corresponding DHP high speed data link parameters was performed until a stable setting was found.

In a second step, the optimization of the voltages and DCD parameters was performed. The goal of this optimisation is to achieve a working point with a large amplification. Increasing the DEPFET gate voltages increases the amplification but also increases the pedestal spread. Figure 6.4 shows the pedestal spread at a gate voltage setting of -1500/$1600 /-1700 \mathrm{mV}$ for the three Gate voltage sections. The histogram on the left side of 


\section{Phase 2 Commissioning}

the figure shows the pedestal spread across the sensor divided into the four DHP-DCD pairs. With these settings the complete dynamic range of the DCD is occupied. As a consequence, pixels with a high pedestal value have only little headroom for signal charges. A total of $1.38 \%$ of the pixels is completely out of the dynamic range (below $1 \mathrm{ADU}$ or above $256 \mathrm{ADU}$ ). The figure also reveals a ring-like structure in the pedestals. The origin of these rings is not fully understood yet. The most likely explanation is a variation of the doping in the wafer from which the sensor matrix is cut. A detailed study of this phenomenon is currently ongoing within the PXD collaboration.

Another visible feature are two broken drain lines, revealing themselves through a series of pixel that have a constant signal value of $1 \mathrm{ADU}$. Because of the design of the drain lines and the rolling shutter, these pixels appear in every fourth row.
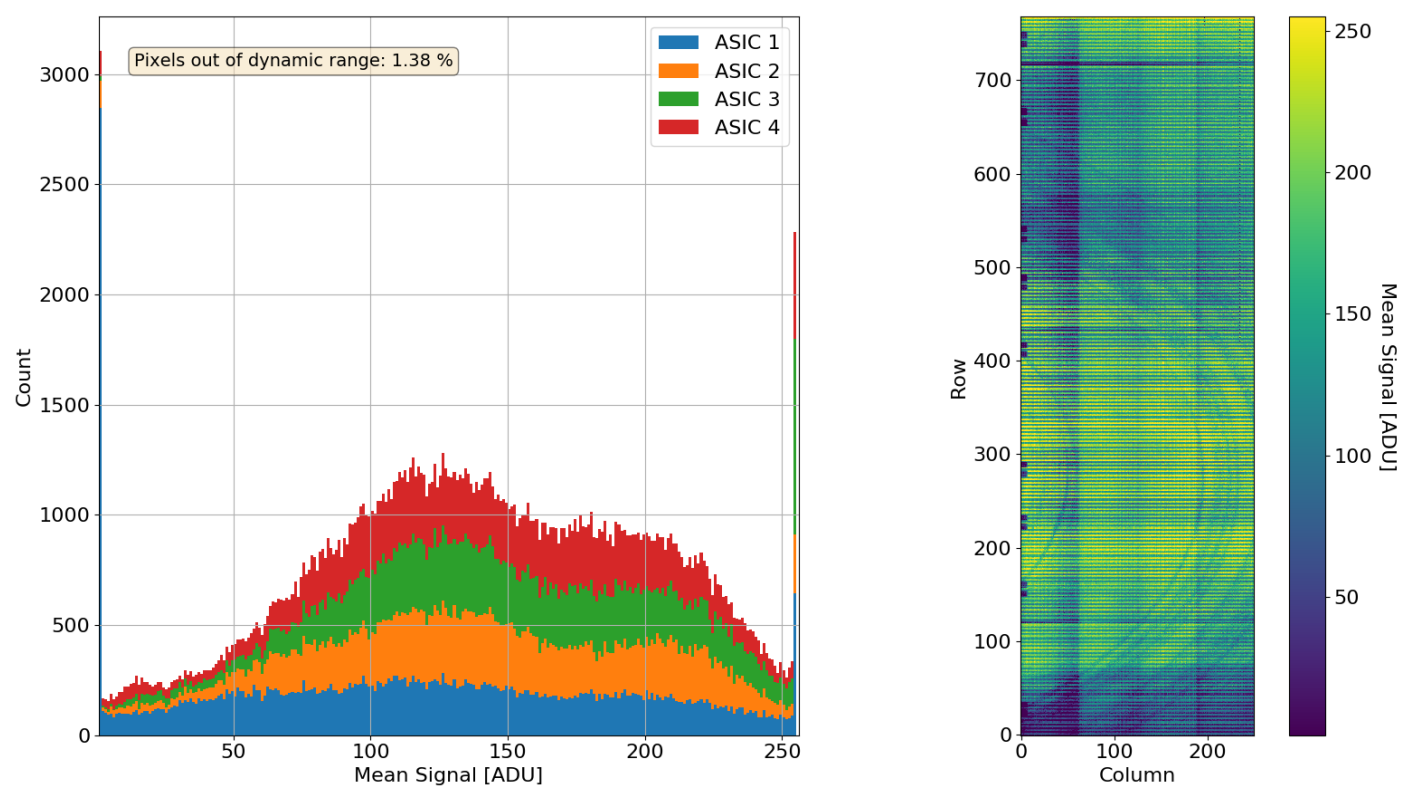

Figure 6.4.: Pedestals after optimization of the Gate-On voltages and the DCD VNSubIn settings. The left plot shows a histogram of the mean signal values (100 data frames). The right plot shows the mean signal value of each pixel as a heatmap.

In order to improve the pedestal distribution and make it narrower, the 2-Bit offset correction was used. Figure 6.5 shows the pedestal distribution with this correction applied. As expected the spread is significantly narrower and the fraction of pixels outside of the dynamic range was reduced to $0.01 \%$.

Due to time constrains no measurements with radioactive sources were performed in Göttingen. Instead the module was shipped to Munich were a short series of measurements with a ${ }^{90} \mathrm{Sr}$ source was performed. Figure 6.6 shows an example of a cluster charge 

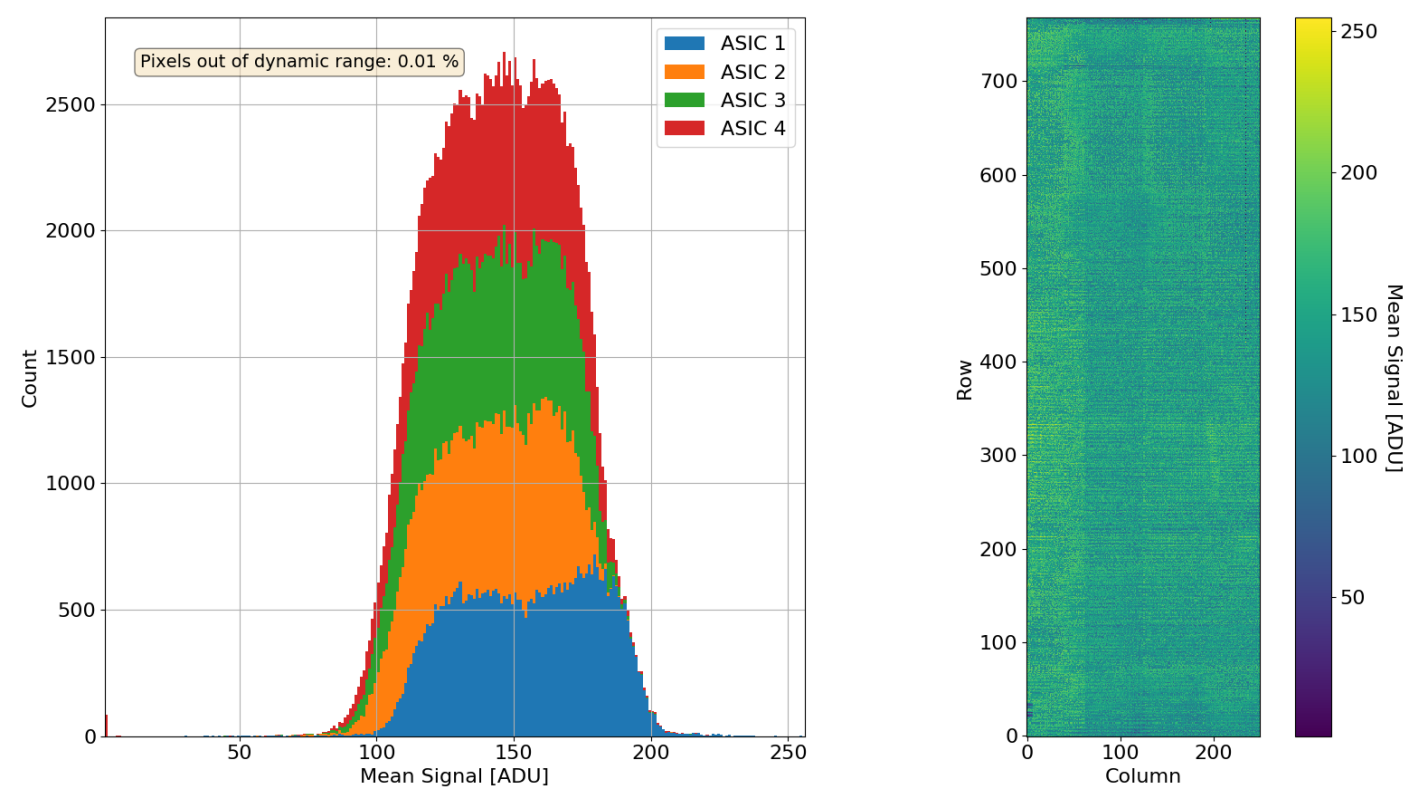

Figure 6.5.: Pedestals with 2-Bit DAC offset correction. The spread of the pedestal distribution is reduced significantly due to the 2-Bit DAC correction.

distribution from these measurements. As ${ }^{90} \mathrm{Sr}$ is a $\beta$-source, the cluster charge distribution can be described by a Landau distribution, convoluted with a Gaussian function to model the resolution of the sensor. 


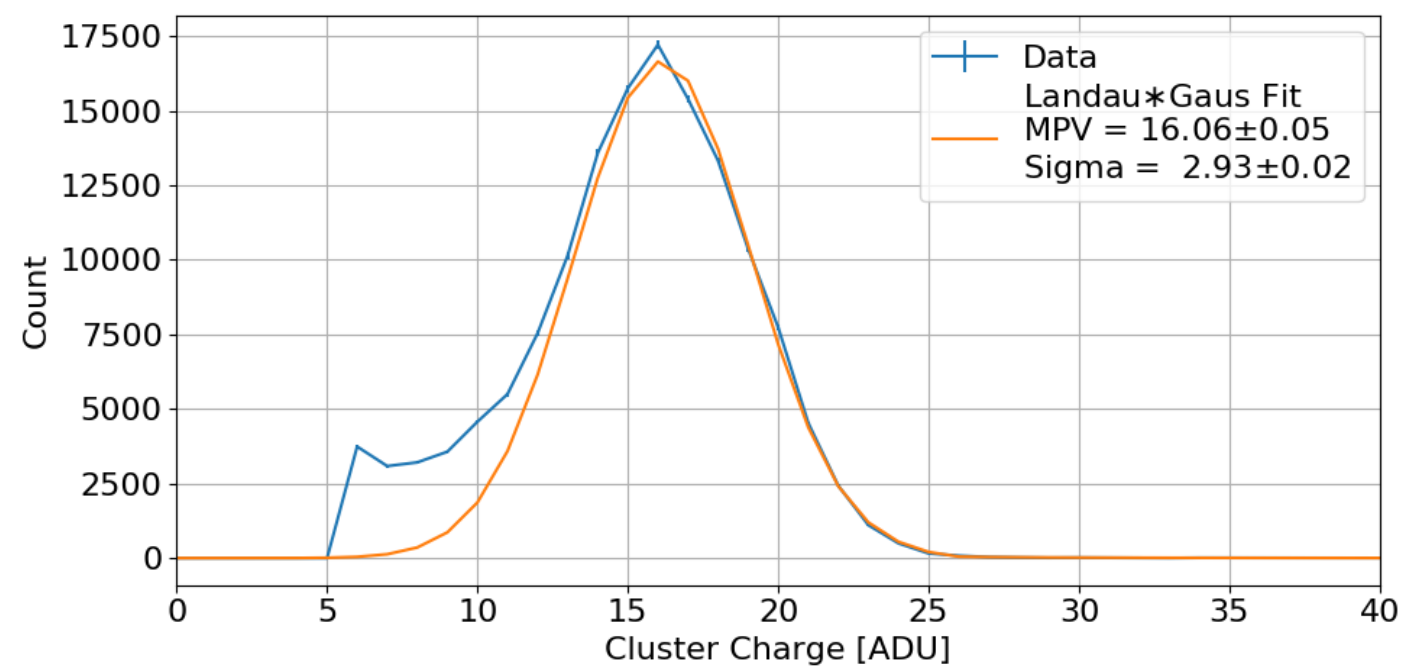

Figure 6.6.: Cluster charge distribution of a ${ }^{90} \mathrm{Sr}$ source measured with the W37_OF1 half-ladder. A landau function convoluted with a Gaussian function is fitted to the data to extract a value for the MPV. The deviation for low cluster charges originate from noise of the sensor.

\subsection{Commissioning and Operation of the Pixel Vertex Detector}

After the testing and characterization of the four half-ladders in Germany, the devices were shipped together with the necessary services to KEK, where they were combined with the SVD ladders and the BEAST 2 sensors to form the VXD volume. During this phase of the commissioning the half-ladders could not be cooled properly, which limited tests to DHP related tests only. The power consumption of the DHP ASIC is low enough so that it can be operated without cooling. In these test the functionality of the DHE $\leftrightarrow$ DHP communication was verified.

As mentioned before, the operation during phase 2 marked the first time four halfladders were operated together. The configuration of the two forward half-ladders of the phase 2 PXD system in terms of power, data and control connections is shown in fig. 6.7. Each half-ladder has its own power supply unit and a DHE/DHI unit. The data streams of the forward and backward sensors were combined in one DHC, which sent out the data to the global Belle II DAQ and an exact copy of this data to the local PXD DAQ. Additionally, the DHCs could be switched into a local mode, where triggers could be generated artificially, which allowed a controlled readout of the sensors to perform calibration measurements. 


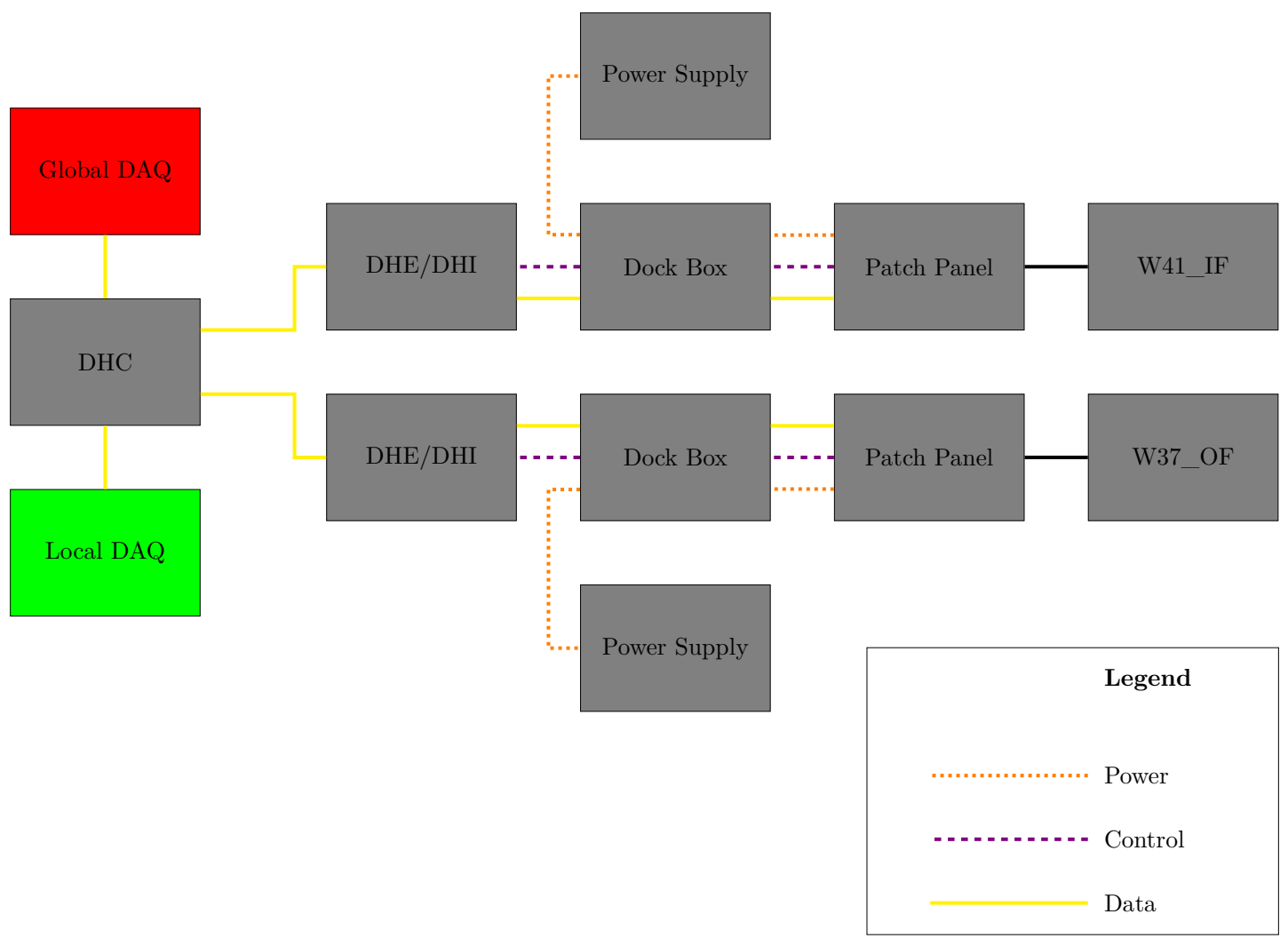

Figure 6.7.: Phase 2 configuration of the two forward half-ladders showing power, control and data line connections. The Kapton flex cable connecting the sensors to their patch panels transfers the data and control signals as well as the power. The data stream sent out by the DHC to the local DAQ and the global Belle II DAQ is identical. For the backward modules the configuration is analogous to the one shown here.

After the installation of the VXD volume in Belle II proper cooling was ensured and further tests of the half-ladders were possible. In this phase the focus was an optimization of the pedestal distributions. Due to different environmental conditions and a different grounding scheme compared to the laboratory, a re-optimization was necessary. This included a recalibration of the 2-Bit offset correction as well. Figure 6.8 shows the pedestal distribution of all four half-ladders after the re-optimization. A direct comparison of the pedestal distibutions shows, that except for the $W 46 \ldots I B$ half-ladder the width of the pedestal distribution was in the range of $150 \mathrm{ADU}$. For the $W 46$ _ IB half-ladder no better 2-Bit offset correction was found. Not only the pedestal distribution was optimized, the correct trigger delay setting on the DHE had to be found, too. 


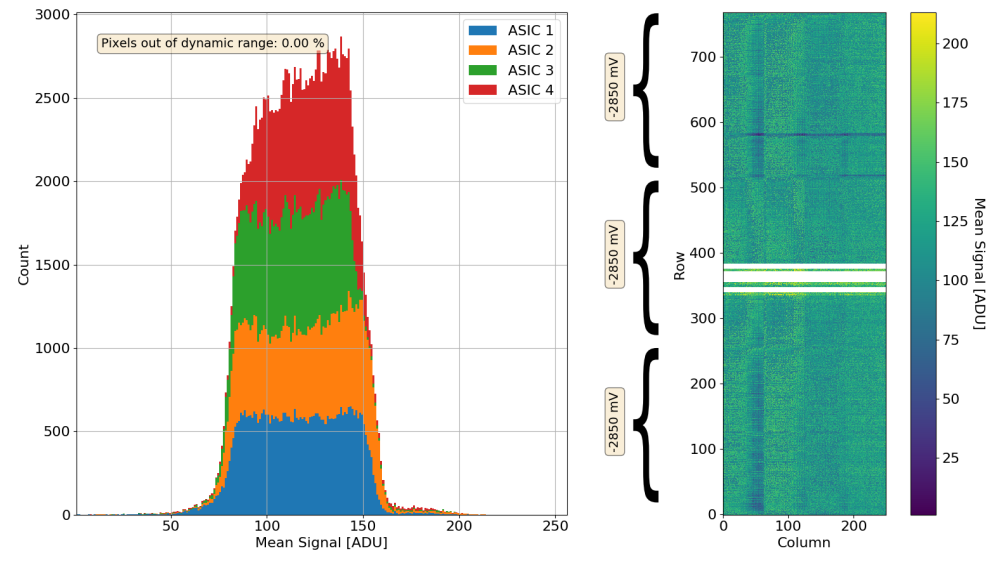

(a) W41_IF

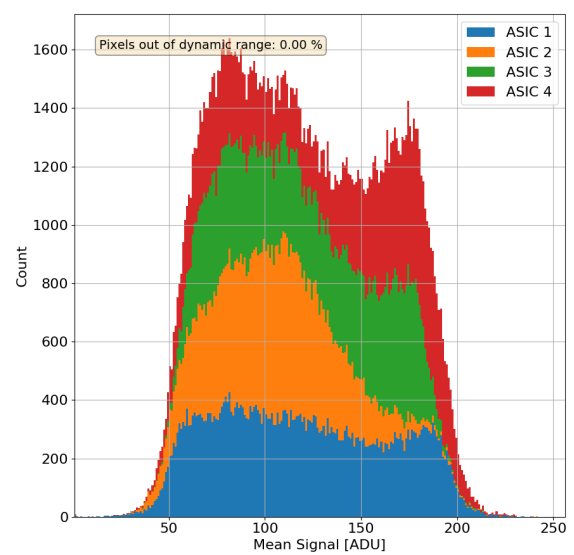

(c) W46_IB

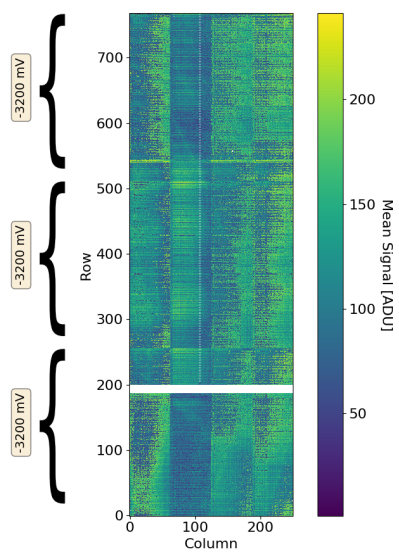

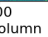

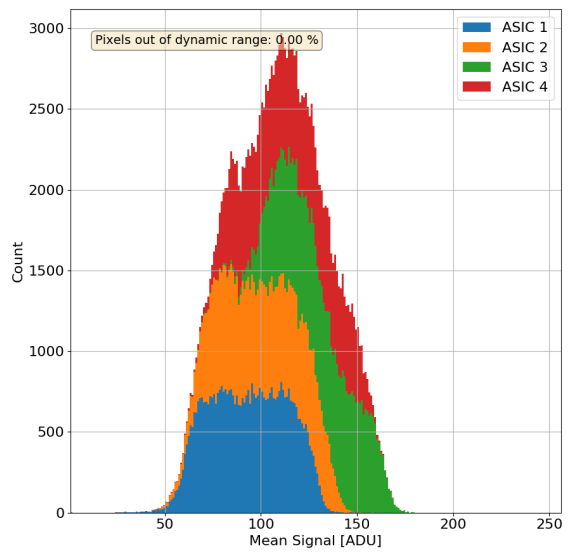

(b) W37_OF
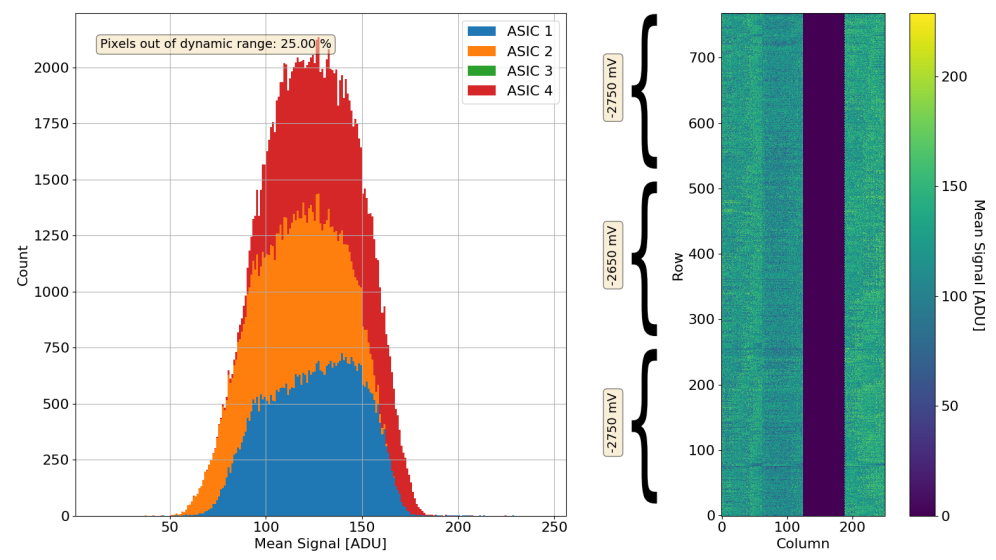

(d) W37_OB1

Figure 6.8.: Pedestals during phase 2 operation. The white rows in the hit maps are rows that were masked deliberately because they were broken or damaged. The highspeed data link of the third DHP on the W37_OB1 sensor was not working properly, which is why this part of the sensor could not be read out. 
Before collisions started, a cosmic run with all subsystems was performed in February 2018 to evaluate the general DAQ performance and to find an optimal trigger delay setting. On the 26th of April, 2018 the first collisions were recorded and the regular phase 2 operation started. During the collisions phase a recalibration of the PXD in regular intervals was necessary to compensate radiation damages on the sensors, see also section 4.4. The most prominent effect was the shift of the DEPFET threshold voltage and therefore the shift of the working point. Due to the shift of the working point the mean drain-source current $\tilde{I}_{D}$ measured by the power supplies showed a clear drop. This current was used as a reference when the gate voltages $V_{G}$ were decreased to compensate the radiation effects.

\subsection{Data Taking Experience and Analysis}

The local DAQ of the PXD was not only used for calibration measurements, but also to record all global Belle II runs independently. Using the same software framework as in the laboratories, it was possible to analyse this data and judge the PXD performance without having to rely on the global Belle II DAQ. The disadvantage of this method being that data from the other sub-detectors like the SVD and CDC was not accessible and no tracking was possible.

\subsubsection{Cluster Charge Analysis}

One type of analysis that does not require information from other subsystems is a study of the cluster charge distributions measured by the PXD sensors and recorded with the local PXD DAQ.

Hot pixel masking: Before the raw data of a run is clustered, a hot pixel masking is performed. The definition of a hot pixel is not precise and varies from analysis to analysis. In general, a pixel is considered hot if it gives a hit signal more frequently than the majority of all other pixels. In case of the PXD sensors this can be due to an electric damage of a pixel or a wrongly uploaded pedestal value. In the analysis presented here pixels were marked as hot in the following way:

1. The occupancy defined as the number of hits divided by the number of data frames (events) for all pixels is calculated.

2. The median of this occupancy distribution is calculated.

3. All pixels that have an occupancy larger than ten times the median occupancy are marked as hot and masked for further analysis.

This calculation is done for each sensor individually.

Clustering After the calculation of the hot pixel mask the hits of neighbouring pixels (including diagonal neighbours) are grouped into clusters. For each cluster the seed pixel is defined as the pixel with the highest hit value of all pixels belonging to the cluster. The charge of the cluster is the sum of the charges of the individual 


\section{Phase 2 Commissioning}

hits. For the clustering a minimum seed charge of $7 \mathrm{ADU}$ is required. This cut is applied to further remove noise hits from the data sample. All further analyses are based on the created cluster database.

A typical cluster distribution of a run during phase 2 can be seen in fig. 6.9. As expected a Landau shaped distribution is visible due to the charged particles traversing the PXD. In addition to this, a second Gaussian shaped component centred around 20 ADU can be seen. When looking at multi-pixel clusters the ratio of the these two components is changed significantly as can be seen in fig. 6.9b. Due to its shape and because of the change in the ratio when comparing multi and single-pixel clusters, this component was identified to be caused by photons. To extract the MPV of the Landau component, a Landau function was fitted to the cluster charge distribution for each run and each PXD sensor. As can be seen in fig. 6.9, these fits describe the rising part of the distribution and the region around the MPV well, but underestimate the number of clusters with larger charges.

This is caused by two effects. First of all the dynamic range of the DCD has to be taken into account when comparing the distributions. Depending on the pedestal value $p_{i}$ of the pixel that gives a signal $s$, the maximum signal is given as $s_{\max }=256-p_{i}$. The second and much more important effect is the angle of the incoming particle. When a particle goes through the PXD under a large angle (compared to perpendicular transition), it deposits more energy on average. As the particles in Belle 2 hit the PXD under a wide range of angles, the cluster charge distribution cannot be described accurately by a single Landau function.

However, the fit and the extracted MPV can be used to evaluate the evolution of the system gain, which changed due to the shifted threshold voltage $V_{\mathrm{thr}}$. If the gate voltage $V_{\text {Gate }}$ is not adjusted, the drain current $I_{D}$ decreases and by that the overall amplification $g_{q}$ :

$$
\left(V_{\text {Gate }}-V_{\mathrm{thr}}\right) \propto \sqrt{I_{D}} \propto g_{q} \propto \mathrm{MPV}
$$

Figure 6.10 shows the evolution of the MPV for all four modules as a function of time. The figure shows that the MPV slowly decreased over time with two pronounced jumps upwards. These jumps occurred whenever the threshold voltage was readjusted and the working point was set back to its original value.

While the threshold shift of the sensors was not measured directly by an I-V curve measurement, the shift can be inferred from the readjustment of the gate voltages. When using the dose measured by the diamond system, the threshold shift can be compared with previous irradiation measurements. This comparison can be seen in fig. 6.11. The comparison shows that the measured threshold shift of the phase 2 sensors is significantly larger than in the previous measurement with a prototype sensor. Even though previous measurements only used DEPFET prototype sensors, the discrepancy seen here 


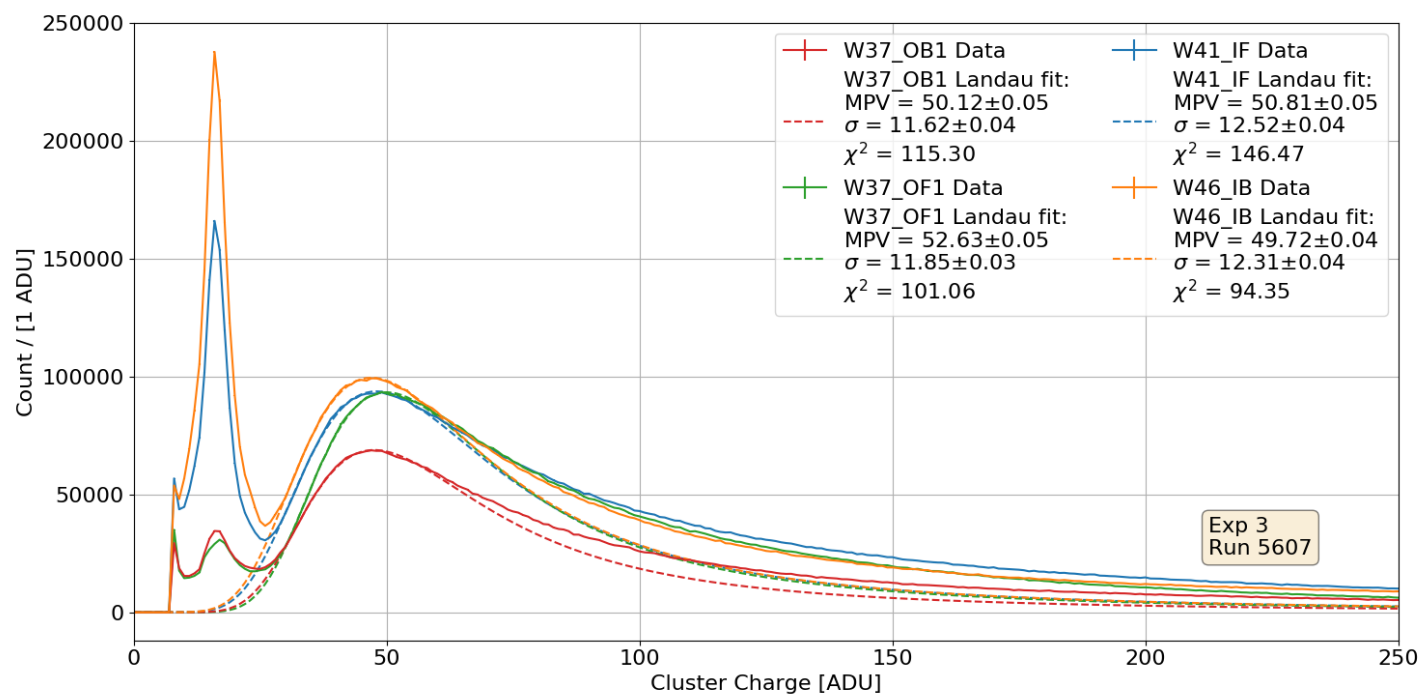

(a) All clusters

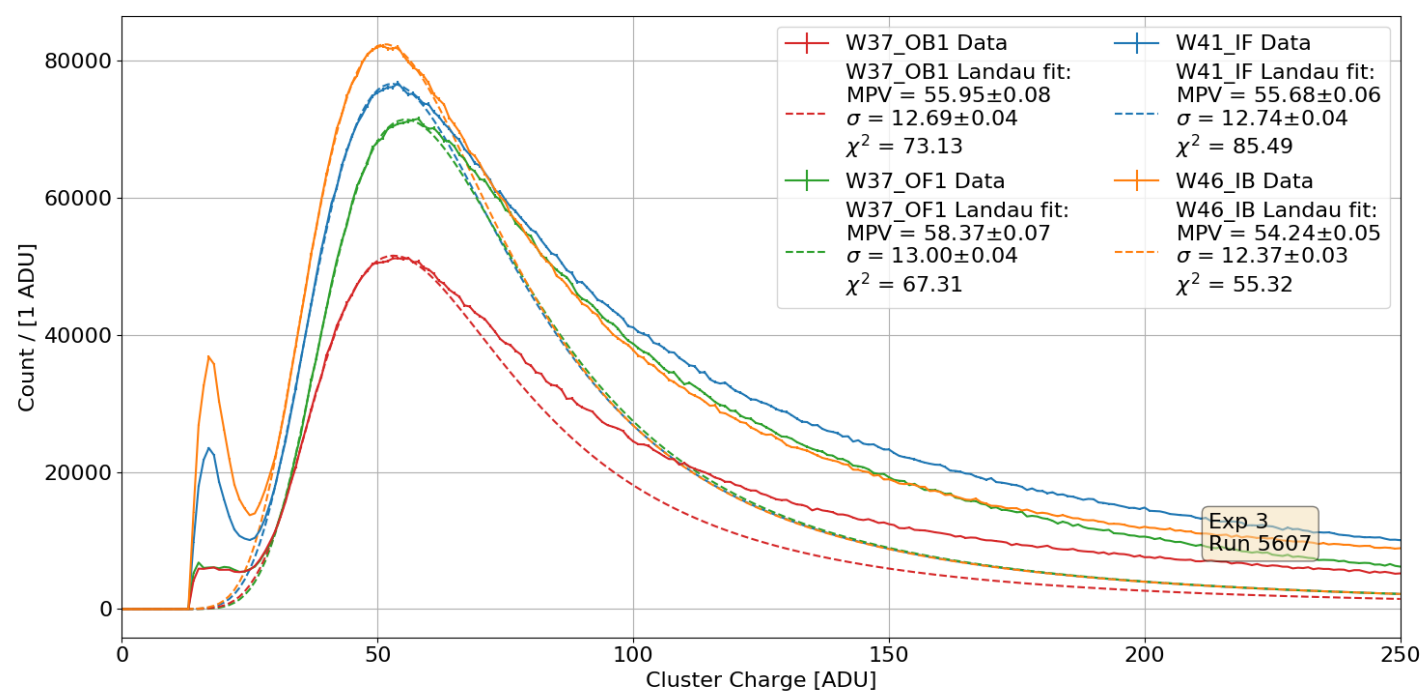

(b) Only multi-pixel clusters

Figure 6.9.: Cluster charge distribution for all four phase 2 sensors. In fig. 6.9a the cluster charge of all clusters is shown and in fig. $6.9 \mathrm{~b}$ only those with a size larger than one. For the outer-backward module (red), the overall entry count is lower because no data was recorded for the third DHP-DCD pair (25\% less data)

is not expected. The most plausible explanation is that the diamond system was not sensitive to some part of the irradiation that affected the PXD and therefore the total 


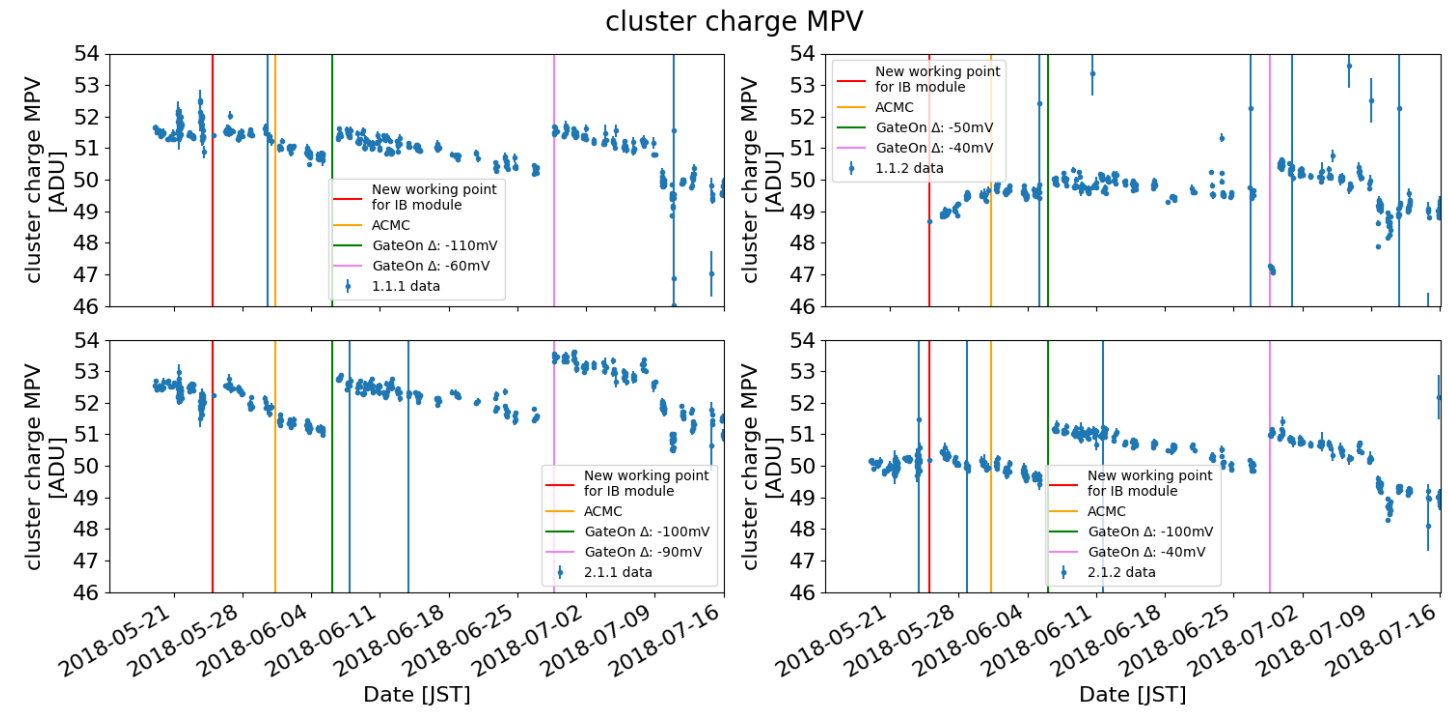

Figure 6.10.: Cluster charge MPV as a function of time for all four PXD sensors: W41_IF (top left), W46_IB (top right), W37_OF1 (bottom left), and W37_OB1 (bottom right). Changes of the applied gate voltages and other changes of sensor conditions are marked by vertical lines.

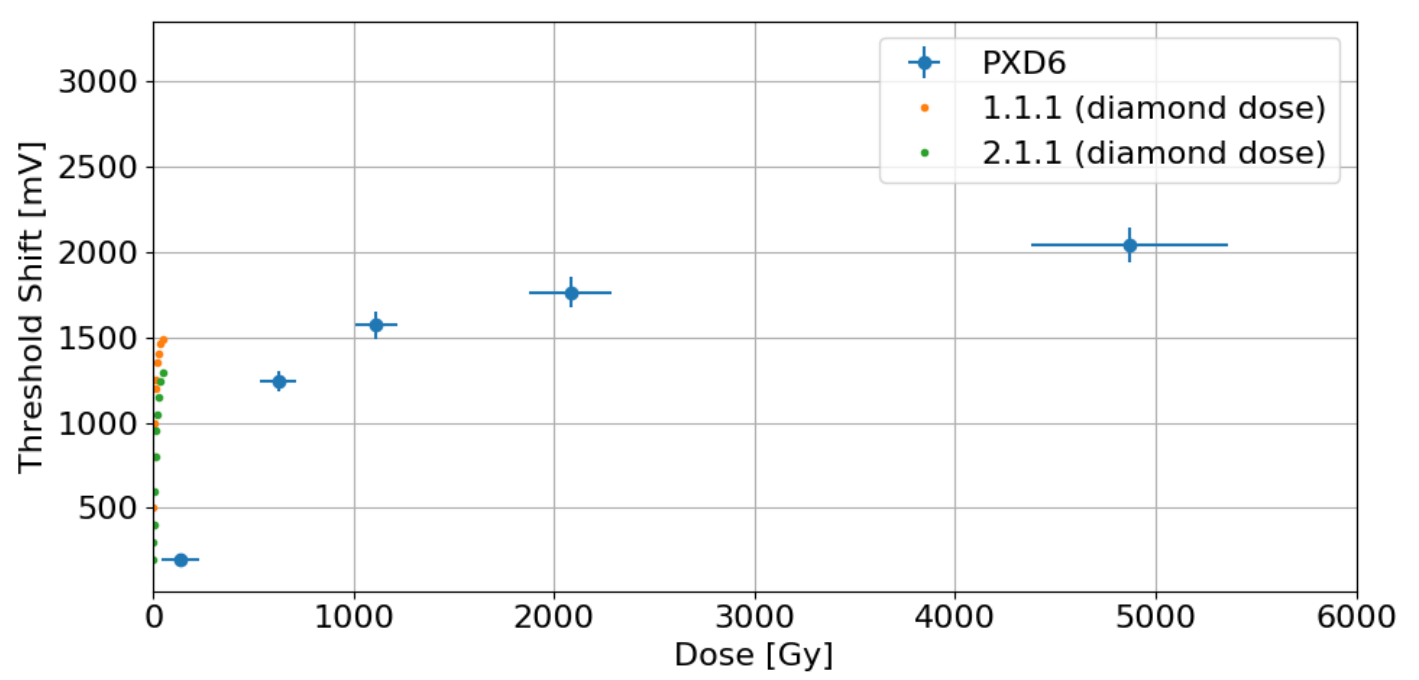

Figure 6.11.: Threshold shift of the phase 2 sensors compared with a previous PXD irradiation measurement. The dose for the phase 2 data points was measured by the diamond system.

dose is underestimated. A naive scaling of the dose of the inner PXD half-ladders by a factor of 100 and 50 for the outer ones aligns the curves reasonable well to the reference 
measurement.

\subsubsection{Sensor Noise}

The PXD sensors rarely show noise levels that would affect their overall performance, especially when the analog and digital common mode corrections are used. To monitor the noise during the phase 2 operation, the PXD local DAQ was configured to record 100 raw data pedestal frames at the end of every run automatically.

Using this data it is possible to look at the pedestal noise, which is defined as the standard deviation of the recorded data frames. Figure 6.12 shows the mean of the noise distribution for all four sensors as a function of time.

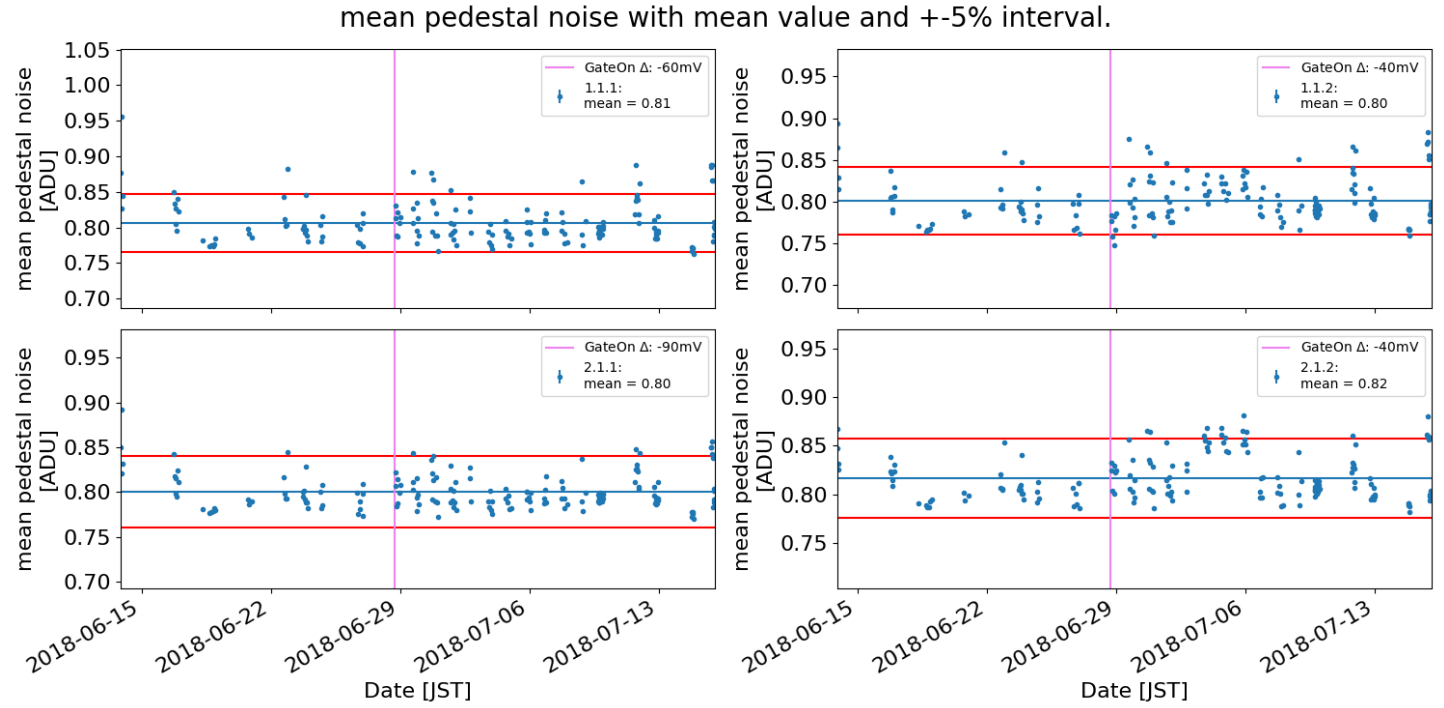

Figure 6.12.: Mean of the pedestal noise distribution as a function of time for all four PXD sensors: W41_IF (top left), W46_IB (top right), W37_OF1 (bottom left), and W37_OB1 (bottom right).

The noise was very stable over the observed timespan and always below 1 ADU, whereas the signal generated by charged particles was around $50 \mathrm{ADU}$ as shown in the previous section.

\subsubsection{Signal-to-Noise Performance}

A key observable of each detector is its signal-to-noise (SNR) value as it allows to judge its overall performance. There are various ways to define a signal-to-noise value for pixel detectors. In this analysis, the SNR is defined in the following way.

1. For each registered cluster a local SNR value of

$$
\mathrm{SNR}_{\text {local }}=\frac{S}{\sqrt{d} \cdot n_{\text {seed }}}
$$




\section{Phase 2 Commissioning}

is defined where $S$ is the total cluster charge, $d$ the size of the cluster and $n_{\text {seed }}$ the pedestal noise at the seed pixel. The pedestal noise is calculated as the standard deviation of 100 raw data frames.

2. The calculated local SNR values are filled into a histogram.

3. A fit with a Landau function to the histogram is performed. As the noise of the sensors is rather homogenous, the SNR distribution can be described by a Landau function. The MPV value of the fit is defined as the global SNR for the sensor.

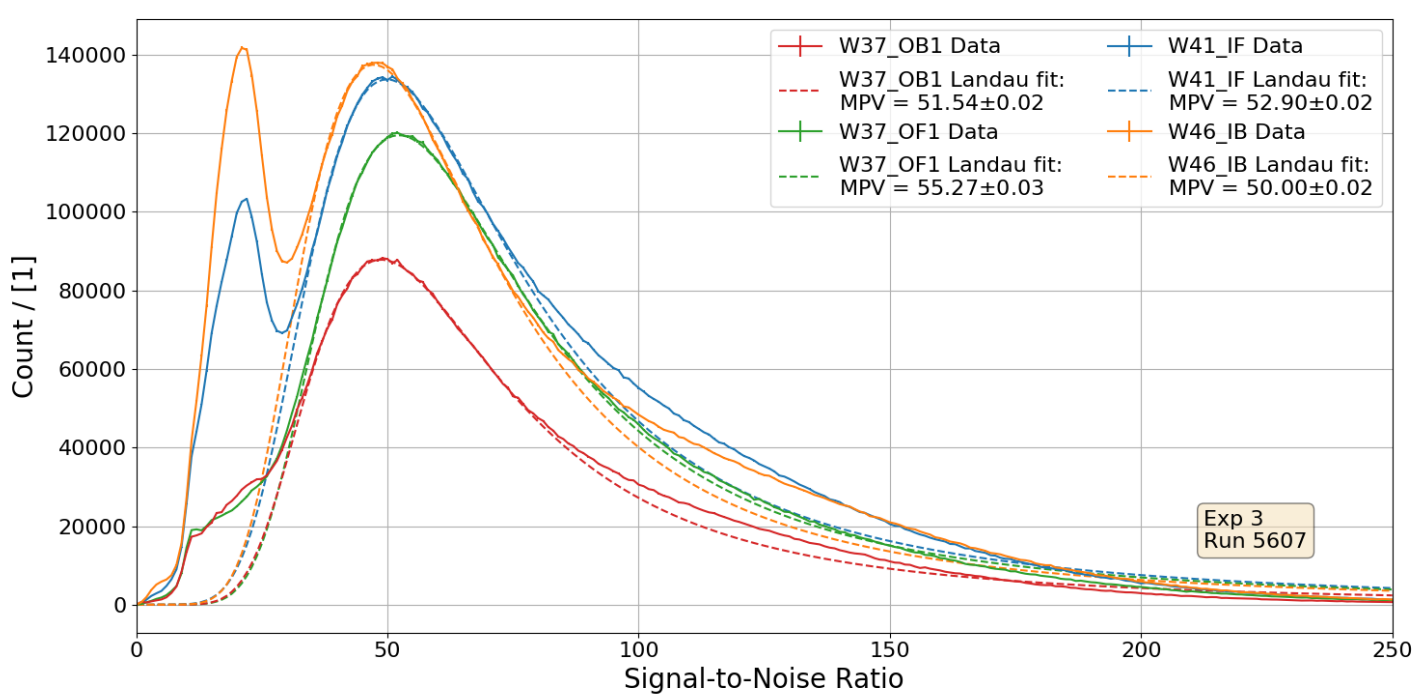

Figure 6.13.: Histogram filled with $\mathrm{SNR}_{\text {local }}$ values of a single run during phase 2. The run had $\approx 1.3$ million events. In addition to the landau shaped component, the photon component is visible for the inner PXD half-ladders.

Figure 6.13 shows the SNR distribution of a run during phase 2 with $\approx 1.3$ million events. For the two inner layers the extra photon component is visible as well in the distributions. For the calculation of the global SNR value this component is not taken into account.

If the data is segmented, the same analysis can be done for individual geometrical regions on the sensor. A natural segmentation are the DCD-DHP pair and switcher regions. As there are four DCD-DHP pairs and six switcher this results in 24 regions. Figure 6.14 shows the SNR calculated individually for these regions and all four PXD sensors. While the SNR is mostly homogeneous across the sensors, some regions show a distinct deviation from the rest. For the W46_IB sensor this is especially apparent. For this sensor one switcher row has a $12 \%$ lower SNR than the other rows. Similar behaviour was also seen in the laboratories on other sensors. 
The pedestal noise on all sensors is very homogeneous and the SNR drop in this particular row is due to a lower amplification of the DEPFETS. As the behaviour can be correlated to one switcher region, the most likely explanation is a shift of the sampling point of this switcher, resulting in a lower signal.

Apart from these singular regions, the SNR was very homogeneous for all runs recorded during the phase 2 operation of the sensor.

Similar to the analysis of the cluster charge MPV evolution, the global SNR value was also calculated for all runs of phase 2. Figure 6.15 shows the SNR of all four half-ladders as a function of time. The evolution shows that all sensors showed an SNR larger than 50 during the whole time. No significant decrease of the SNR is visible. 


\section{Phase 2 Commissioning}

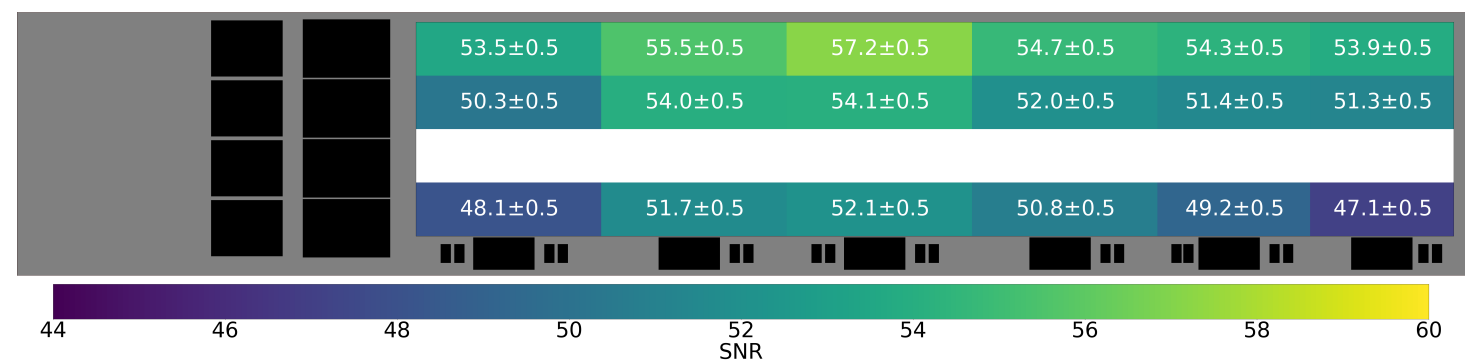

(a) $\mathrm{OB}$

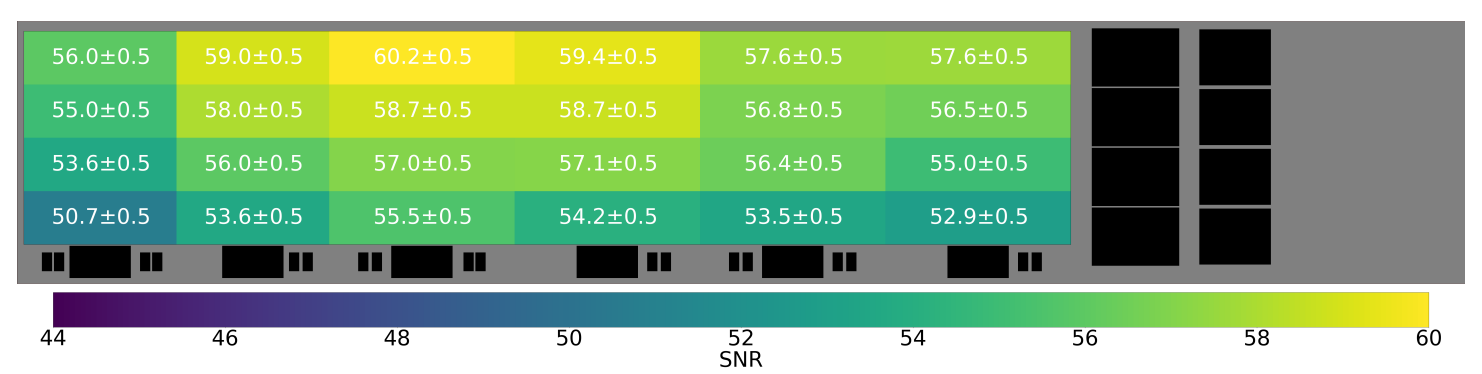

(b) $\mathrm{OF}$

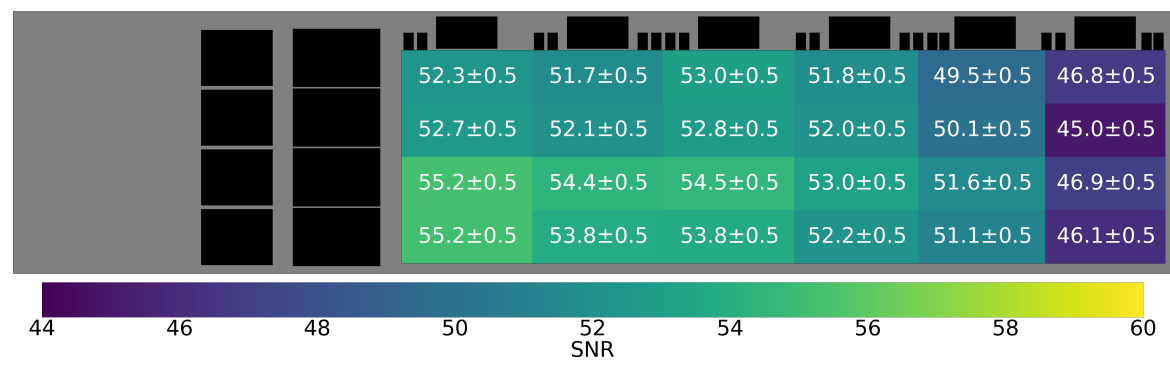

(c) IB

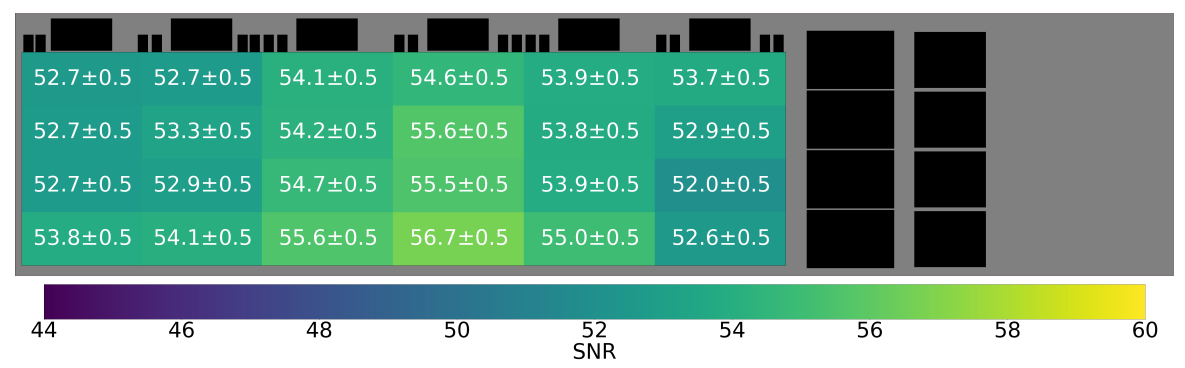

(d) IF

Figure 6.14.: SNR values of all four phase 2 PXD sensors as maps (run 6288). Each sensor is divided in 24 ASIC-Switcher regions. The figures use the correct scales and the ASICs are shown to visualise the orientation of the sensors. 
Signal-to-noise MPV with mean value and +-5\% interval.
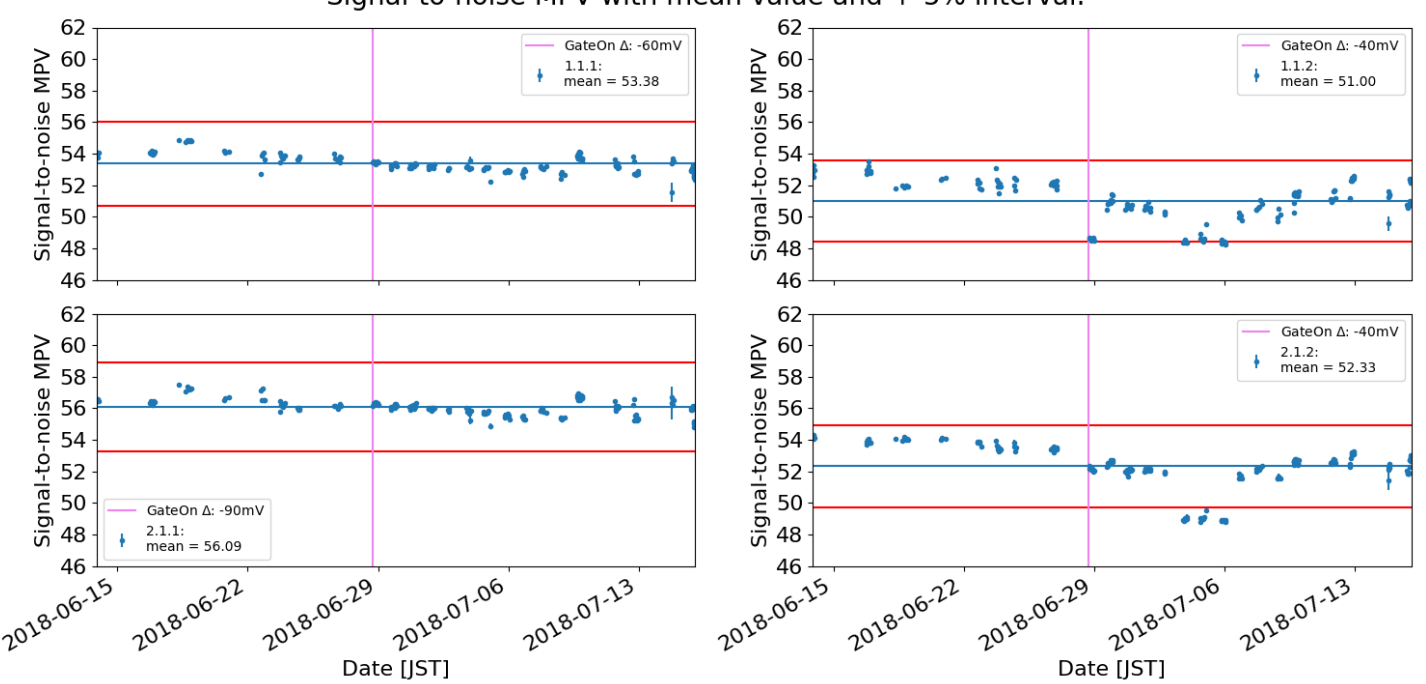

Figure 6.15.: Signal-to-noise ratio evolution for all four PXD sensors: W41_IF (top left), W46_IB (top right), W37_OF1 (bottom left), and W37_OB1 (bottom right). The mean (blue line) as well as the $\pm 5 \%$ is indicated for each halfladder individually. The drop for the backward modules shortly before 2018-07-06 is due to a misconfiguration of the system at this time. 


\section{Phase 2 Commissioning}

\subsection{Total Ionising Dose Estimation}

The threshold shift seen on the phase 2 PXD sensors indicates that the total ionising dose is significantly higher than what was measured by the Diamond Radiation Monitoring and Beam Abort System. Using only the clusters in the PXD it is possible to calculate a 'PXD dose rate'. In order to do that an energy calibration of the data was performed. For this calibration the medians of the measured cluster charge distribution of the PXD and simulated distributions were compared to calculate a conversion factor. Assuming MIPs (minimal ionising particles) as the particles going through the PXD the dose per cluster was calculated and then further processed to get a data sample with a mean dose rate per second solely based on PXD data. For this calculation one has to take into account that each "second" is only partly filled with PXD data, see fig. 6.16. Each time the PXD is triggered, it is read out for $20 \mu$ s (rolling shutter). The coverage of

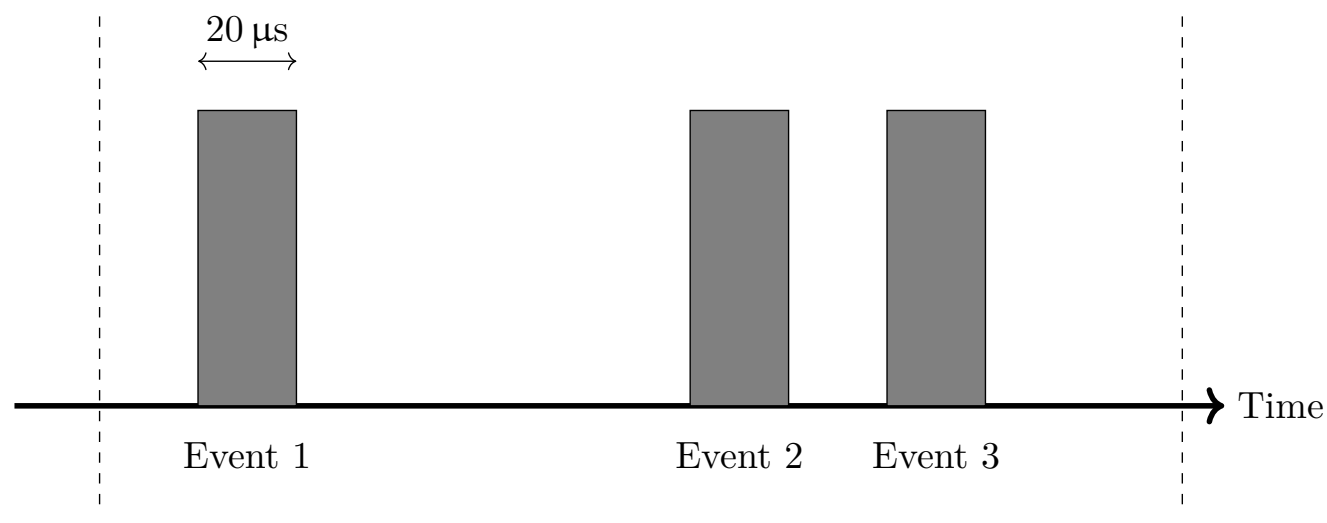

$\mathrm{n}$ th second

$\mathrm{n}+1$ th second

Figure 6.16.: For each trigger/event the PXD sensors have a readout time of $20 \mu$ s (time of rolling rolling shutter). When the data is split into one second intervals and the mean dose rate is calculated this has to be considered as each second is only "partly" read out.

each second is therefore $n \times 20 \mu$ s, when $n$ is the number of events in the one second window. The calculated dose rate is corrected accordingly. One disadvantage of this method is that this approach assumes that there is no significant difference between triggered and un-triggered time spans. As the luminosity during phase 2 was lower by a factor of $\approx 1000$ compared to the design luminosity of $8 \cdot 10^{35} \mathrm{~cm}^{-2} \mathrm{~s}^{-1}$, this approach is justified.

Neglecting the luminosity related backgrounds, the remaining components are Touschek and beam-gas (direct synchrotron radiation hits on the PXD unlikely because of the design of the interaction region). As explained in section 3.1.1, the occupancy (and therefore the dose rate) of the PXD can be described by a heuristic model with a linear 
beam-gas and a quadratic Touschek component as

$$
D_{\mathrm{PXD}}=\sum_{x \in\{\mathrm{LER}, \mathrm{HER}\}} B_{x} Z^{2} P \cdot I_{x}+\frac{T_{x}}{\sigma_{y} n_{b}} \cdot I_{x}^{2},
$$

where $P$ is the vacuum pressure, $\sigma_{y}$ the vertical beam size, $n_{b}$ the number of bunches, $Z$ the atomic number of the beam gas, $I$ the beam current, $B$ the beam-gas coefficient and $T$ the Touschek coefficient. For single beam operation eq. (6.3) simplifies and can be transformed to a linear equation:

$$
\frac{D_{\mathrm{PXD}}}{Z^{2} P \cdot I}=B+\frac{T}{\sigma_{y} n_{b} Z^{2} P} \cdot I
$$

This linearisation has the advantage of allowing an easier and more stable fitting to the recorded data. Figure 6.17 shows the evolution of PXD dose rate during several single beam runs (only LER) together with the heuristic beamgas + Touschek model. The fit parameters have been determined for each sensor individually. During phase 2 several
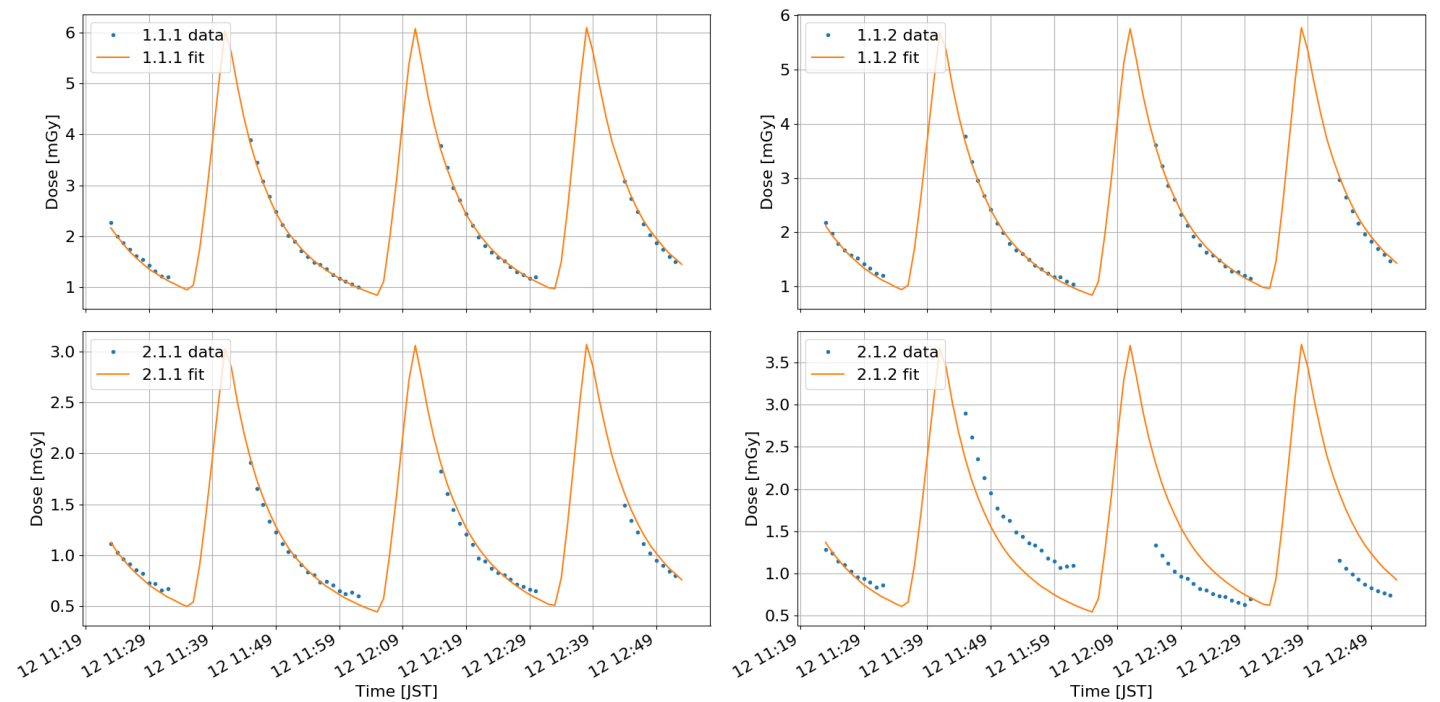

Figure 6.17.: Comparison of measured PXD dose rates with heuristic fit during single beam operation (LER). All phase 2 sensors but the 2.1.2 (outer backward) show a good agreement with the heuristic beamgas + Touschek model.

single beam studies have been performed and for all of them a good description using the heuristic model can be found. Depending on the used beam optics and emittance settings, the beamgas and Touschek parameters vary.

Because the PXD was not always read out during the operation of the detector there are gaps in this dataset. For the timespan between 2018-03-19 and 2018-07-17 PXD data is available for $\approx 12 \%$ of the time. To fill these gaps the data from the diamond 


\section{Phase 2 Commissioning}
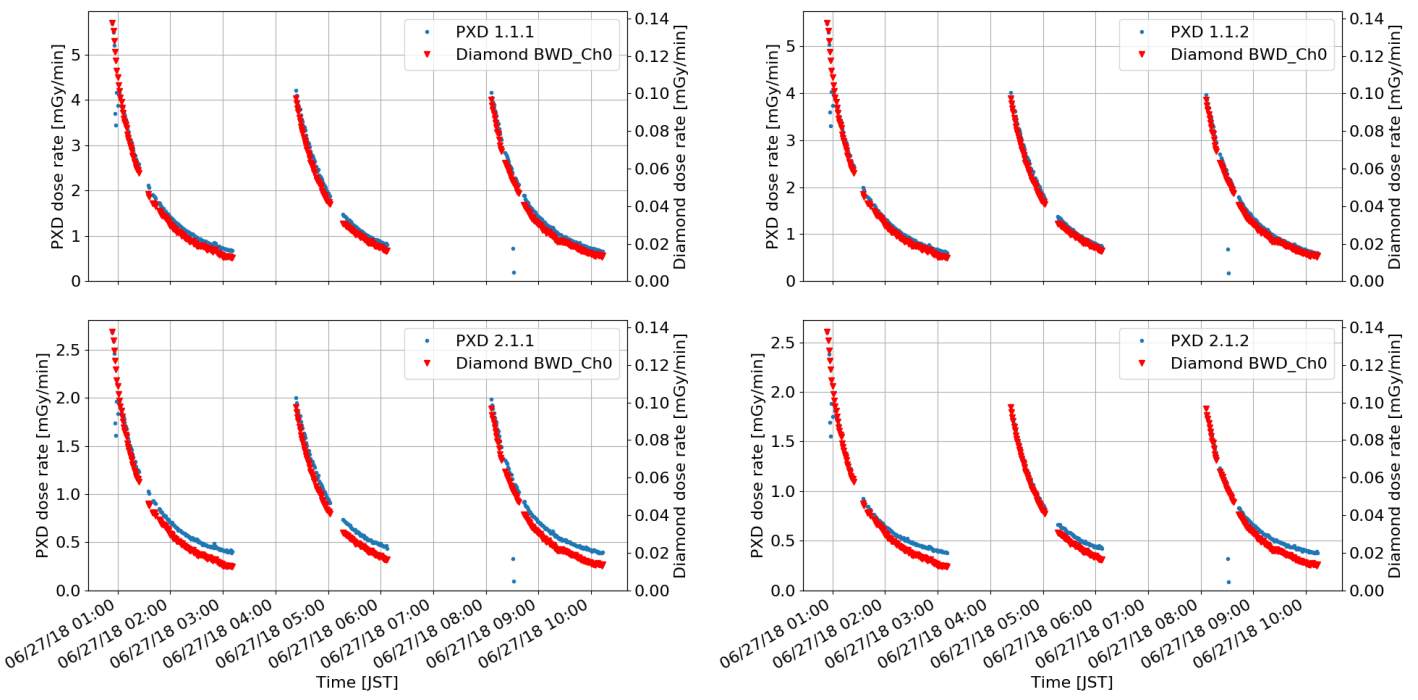

Figure 6.18.: PXD (blue) and Diamond (red) dose rates over 24 hours during phase 2 . The four sub figures show the four PXD sensors used during phase 2. The diamond dose rate is in all sub figures the same.

system is used. To do this, it is necessary to understand how the dose rate measured by the diamond system is related to the PXD dose rate. Looking at fig. 6.18 one can see how the the dose rates measured by the PXD and the diamond system show a very similar behaviour but differ significantly in their absolute values. The gaps seen in this figure correspond to times when the PXD was switched off and not read out because of injections into the accelerator. The diamonds were active the whole time but the data is not shown in this figure. The measured dose rates decrease over time due to the decreasing beam currents in the accelerator.

To compare the two dose rates in more detail their ratio was calculated. Figure 6.19 shows the ratio PXD/diamond. Here it can be clearly seen that the ratio is not constant over time. Instead it rises over the course of each run. Another observation made here is that the rates for the inner and outer modules differ. While the inner modules show a ratio between 30 and 50, the outer modules show a smaller ratio between 15 and 30, which is expected as the inner modules are affected more strongly by the aforementioned photon component of the background radiation than the outer modules.

The observables that change most quickly during a run are the beam currents. A connection between the beam currents becomes even more apparent when the PXD/diamond ratio is plotted against the beam current of the low and high energy ring (LER/HER). The dependency of the ratio $R$ on the beam current $(x)$ can be described reasonably 

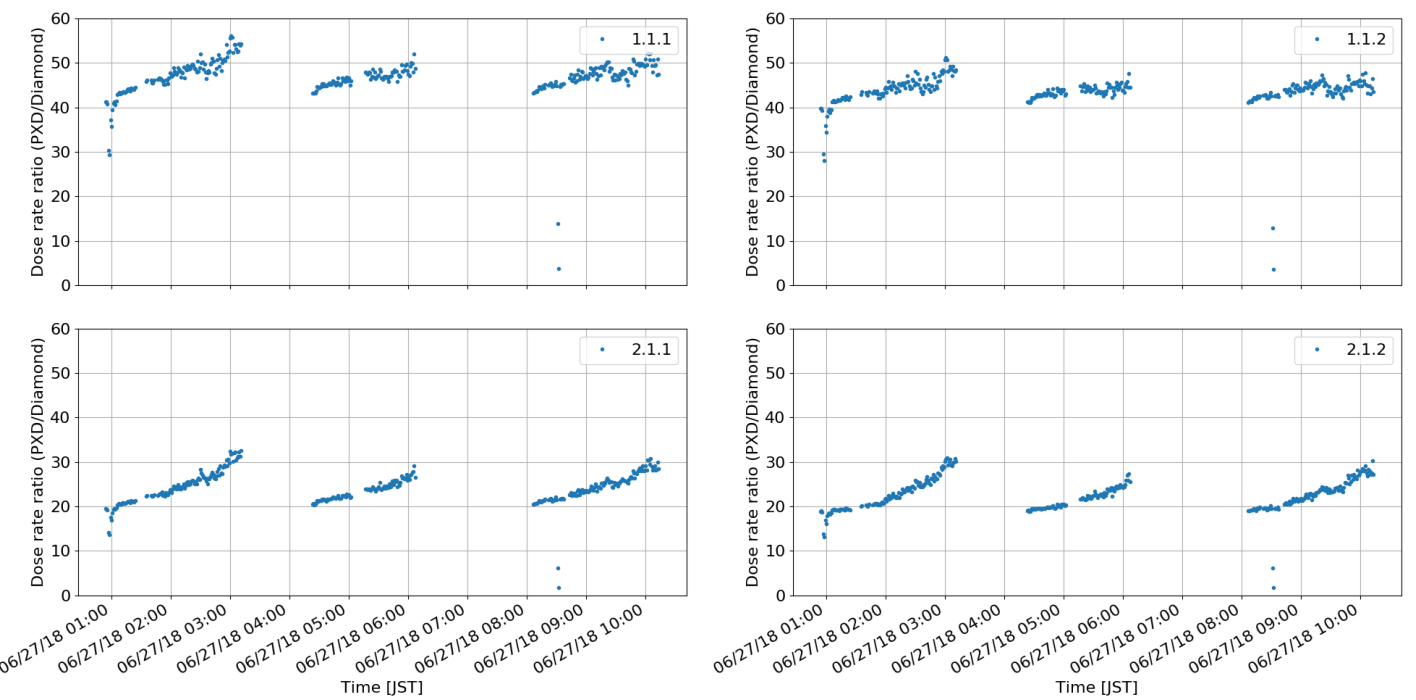

Figure 6.19.: Ratio of the PXD/diamond dose rates for all four PXD modules. The inner modules show on average a higher ratio than the outer modules. All modules have in common that the ratio rises during a run. The ratio shown is calculated with only one of the eight diamond channels.

well by an exponential function of the form

$$
R(x)=a \cdot e^{-b \cdot x}+c
$$

where $a, b$ and $c$ are parameters determined by a fit to the data. Figure 6.20 shows the ratio as a function of the HER and LER beam currents together with the fit of the exponential function. The fit was done for each PXD module and each diamond channel individually with a dataset of one day. This partition of the data is necessary because the parameters of the machine, the diamonds and to some degree the PXD changed during phase 2. Looking at fig. 6.21 which shows the evolution of one of the fit parameters one can see that 3 different zones can be identified:

- Zone 1: From 2018-05-26 to 2018-06-01

- Zone 2: From 2018-06-02 to 2018-07-01

- Zone 3: From 2018-07-02 to 2018-07-10

Because the fit gives similar results within the individual zones, a mean is calculated for each fit parameter and a lookup table is created for each module, diamond channel and fit parameter. Based on this table, eq. (6.5), the diamond dose rate and the beam currents a PXD dose rate $D_{\mathrm{PXD}}^{\prime}$ can be calculated to fill the gaps where the PXD was not sending data.

$$
D_{\mathrm{PXD}}^{\prime}\left(x, D_{\text {Diamond }}\right)=D_{\text {Diamond }} \cdot R(x)
$$




\section{Phase 2 Commissioning}

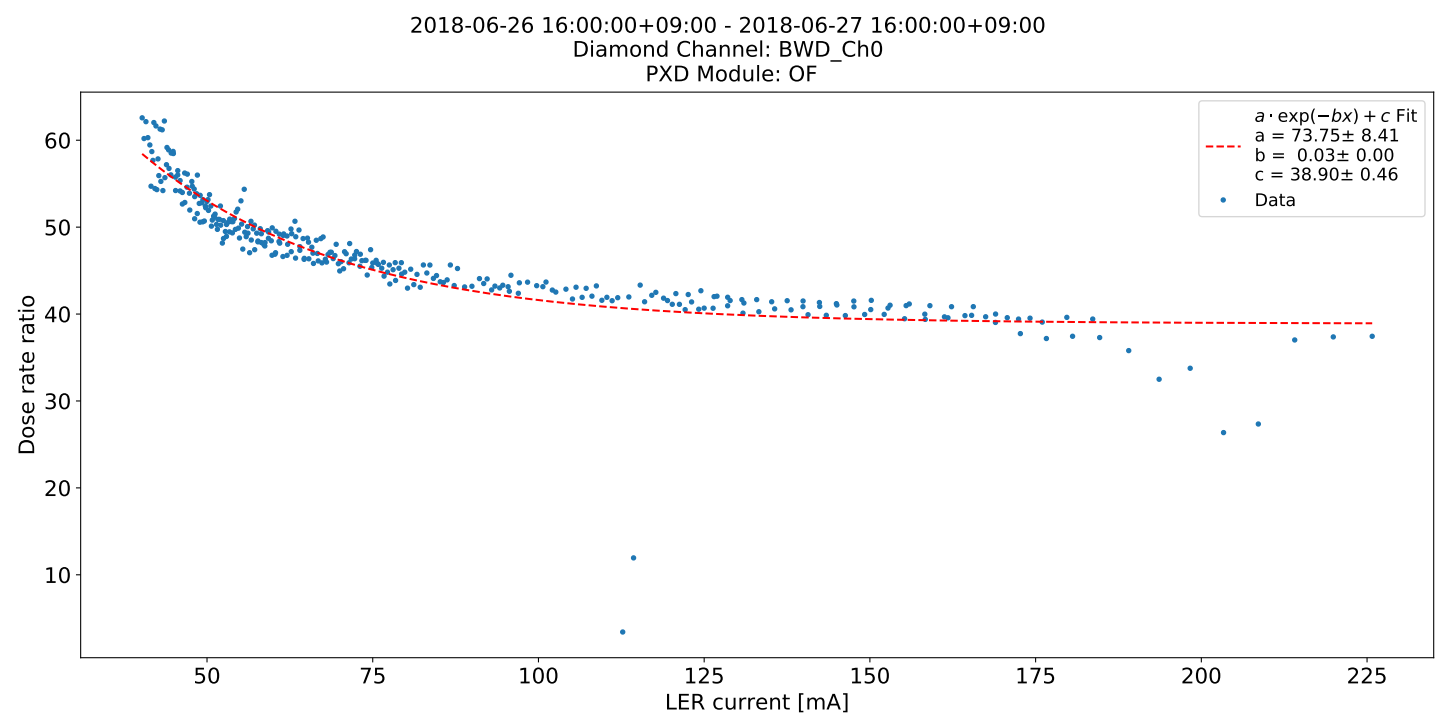

(a) LER

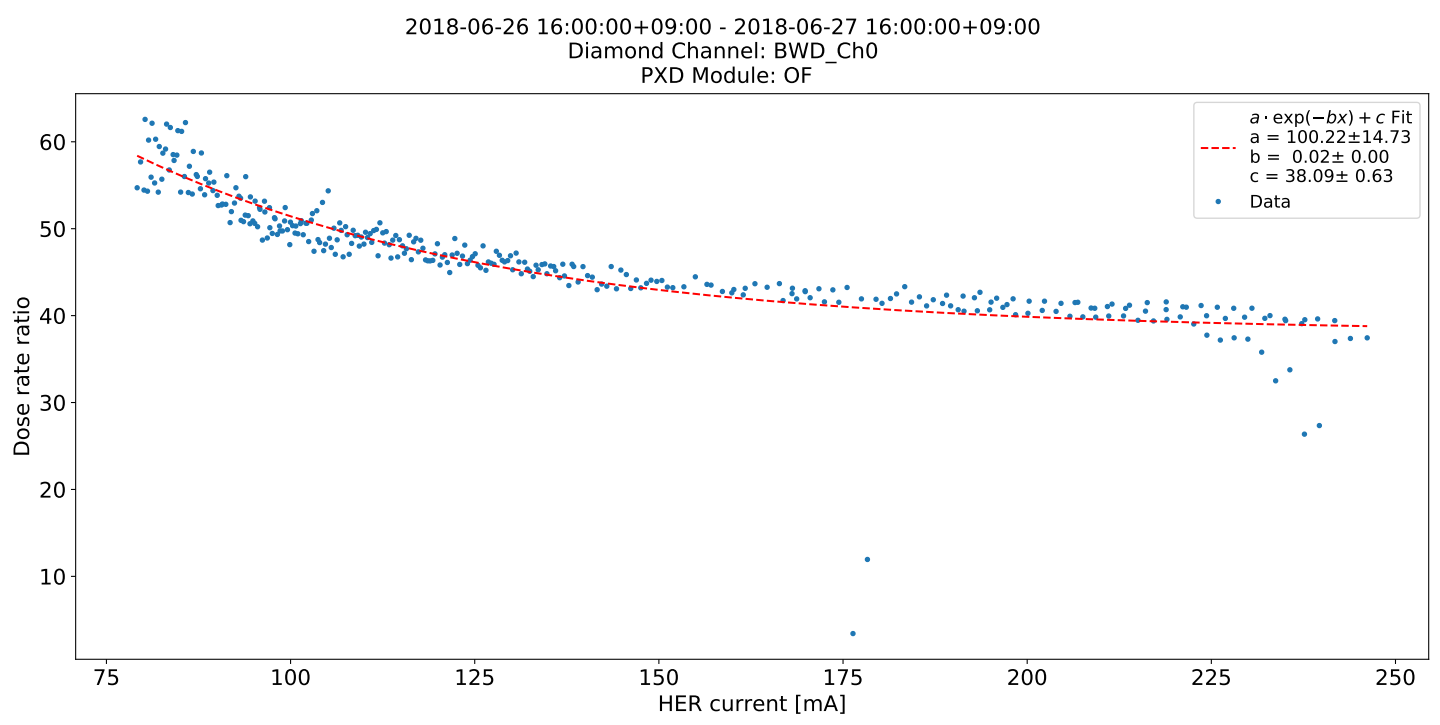

(b) HER

Figure 6.20.: PXD/Diamond ratio plotted as a function of the beam currents. 


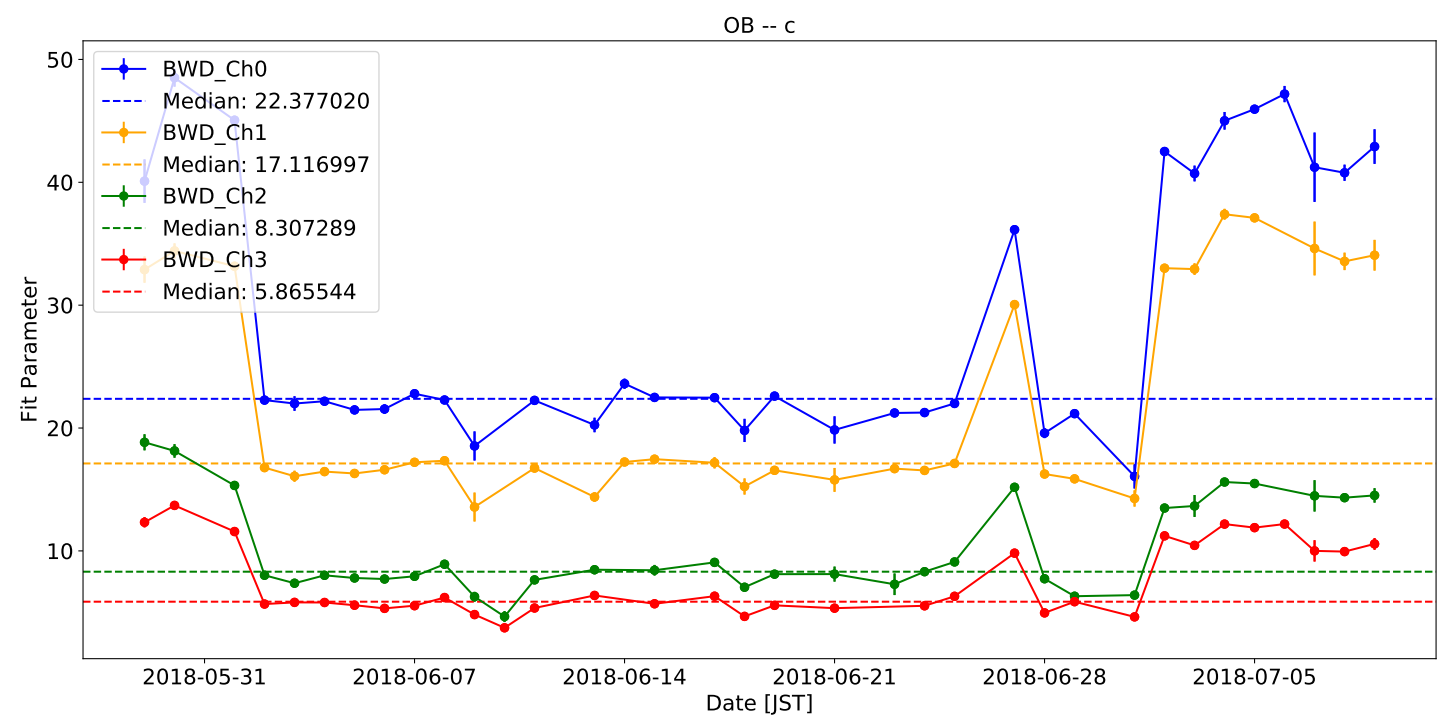

Figure 6.21.: Evolution of the fit parameter $c$.

The calculation is done for each diamond channel separately and the mean for each PXD sensor $j$ is calculated:

$$
D_{j, \mathrm{PXD}}^{\prime}\left(x, D_{\text {Diamond }}\right)=\frac{1}{4} \sum_{i=1}^{4} D_{i \text {, Diamond }}^{\prime} \cdot R_{i j}(x)
$$

To evaluate how accurate these methods are in comparison to the dose actually measured by the PXD, the relative difference between $D_{\mathrm{PXD}}$ and $D_{\mathrm{PXD}}^{\prime}$ was looked at for each zone and each module. Figure 6.23 shows the result for the whole range of phase 2. Figures that show the residuals for the other zones can be found in appendix B.1.

Figure 6.24 shows the cumulated sum of the PXD dose rates restricted to the points in time where the PXD was switched on and sending data. With this restriction one can see that $D_{\mathrm{PXD}}^{\prime}$ agrees nicely with $D_{\mathrm{PXD}}$. All of the previous plots show a comparison of the calculated and measured PXD dose rates. To calculate the total dose of phase 2 these data sources are combined. Because of it being the direct measurement, $D_{\text {PXD }}$ has the highest priority and is used whenever it is available, gaps in the time series are filled with $D_{\mathrm{PXD}}^{\prime}$. The combination $D_{\mathrm{PXD}}^{*}$ gives the best estimation of the PXD dose rate and allows to give a number on the total dose of phase 2 seen by the PXD. Figure 6.25 shows the evolution of $D_{\mathrm{PXD}}^{*}$.

At the end of phase 2 the total dose received by the PXD half-ladders is $\approx 2000 \mathrm{~Gy}$ and $\approx 1000 \mathrm{~Gy}$ for the inner and outer sensors, respectively. Using the calculated dose $D_{\mathrm{PXD}}^{\prime}$ it is possible to interpret the observed threshold voltage shift on the sensors as a function of received dose. Figure 6.26 shows the received dose the inner forward (W41_IF) and the outer forward (W37_OF1) sensor compared with previous PXD 


\section{Phase 2 Commissioning}

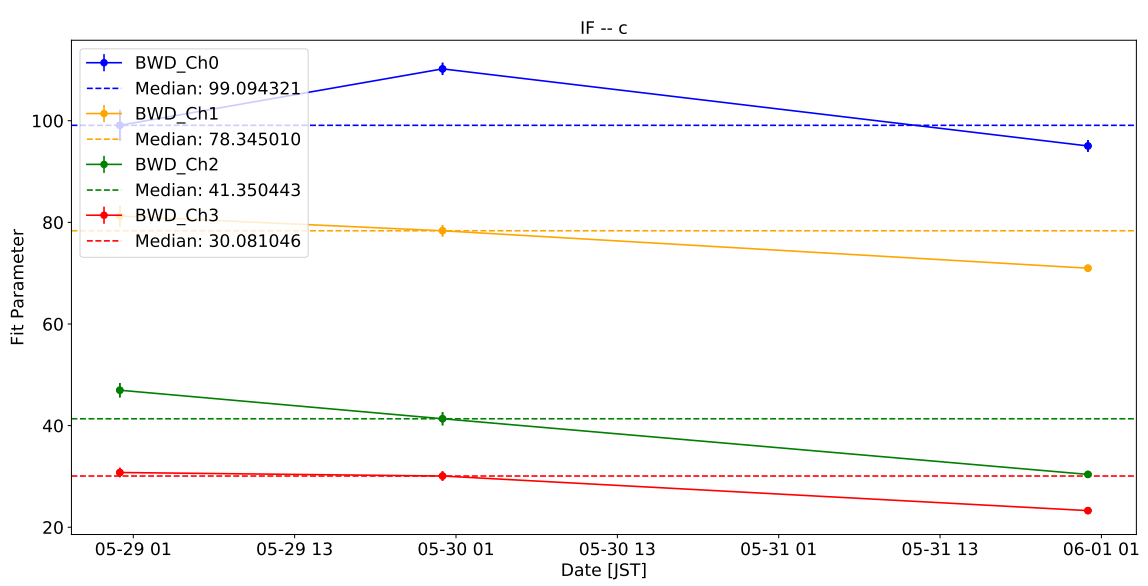

(a) First zone.

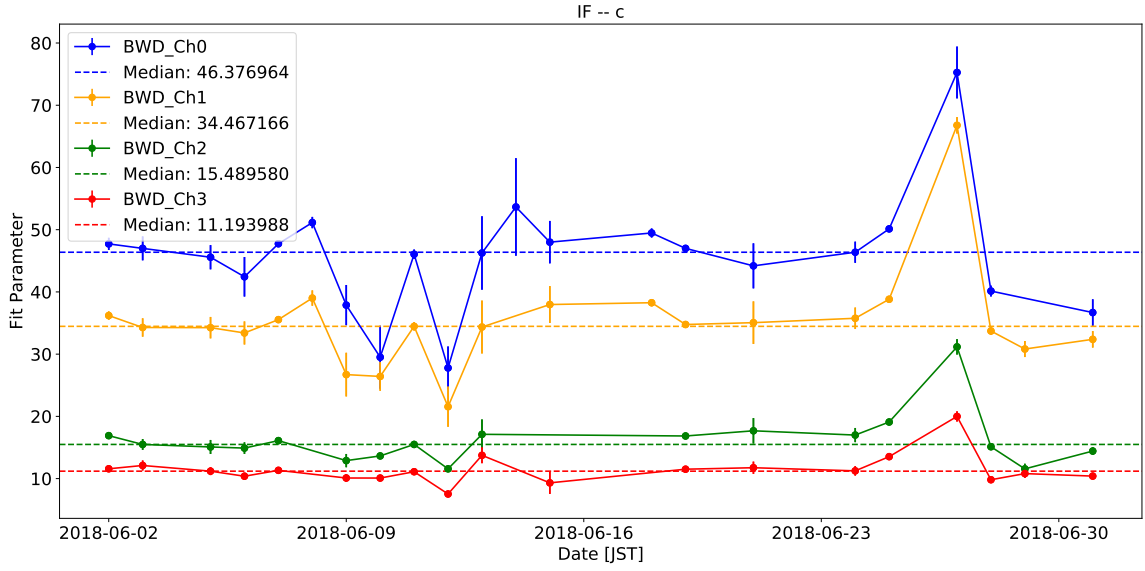

(b) Second zone.

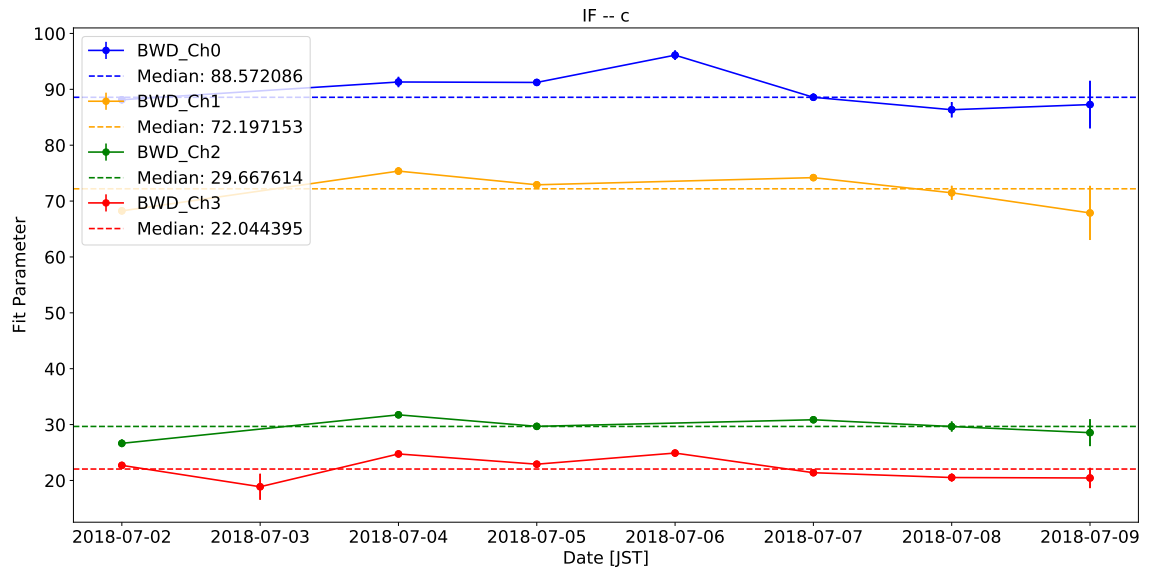

(c) Third zone.

Figure 6.22.: Mean of fit parameters for the individual zones for the inner forward PXD module. 

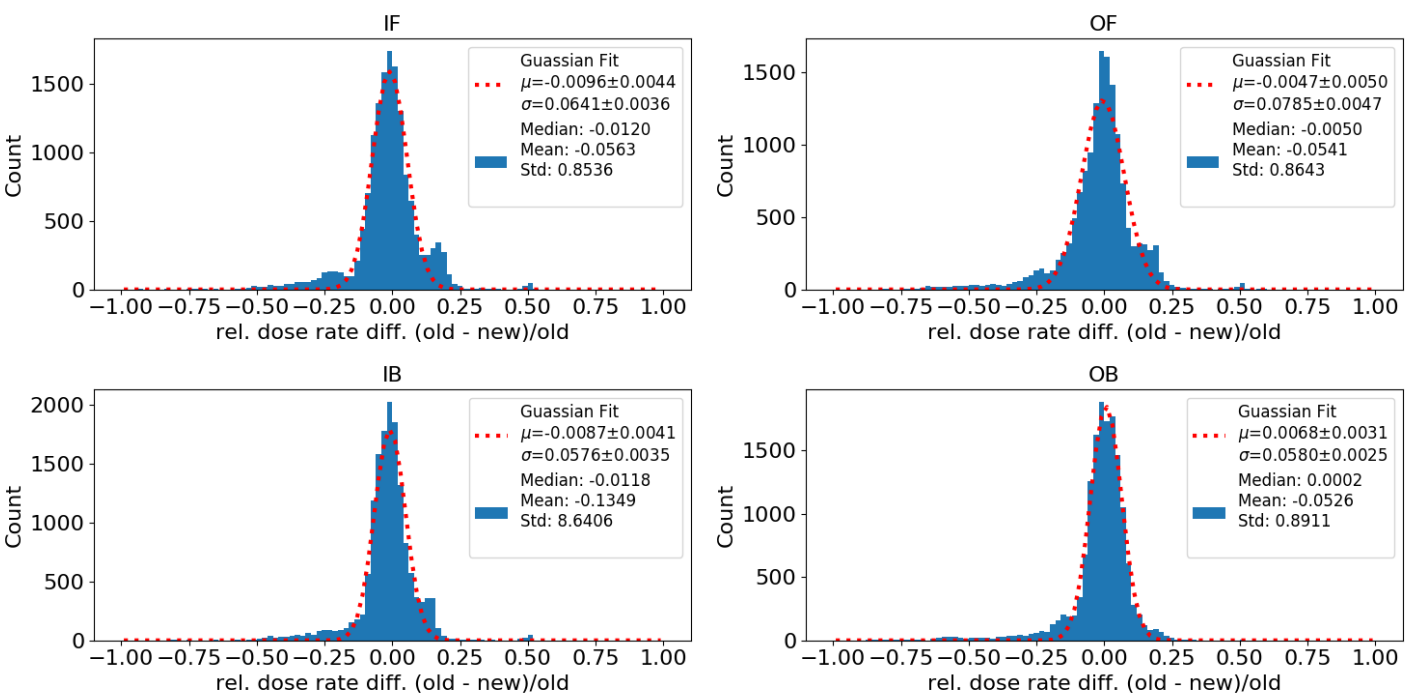

Figure 6.23.: Calculated Residuals for the entire dataset and all four phase 2 sensors.
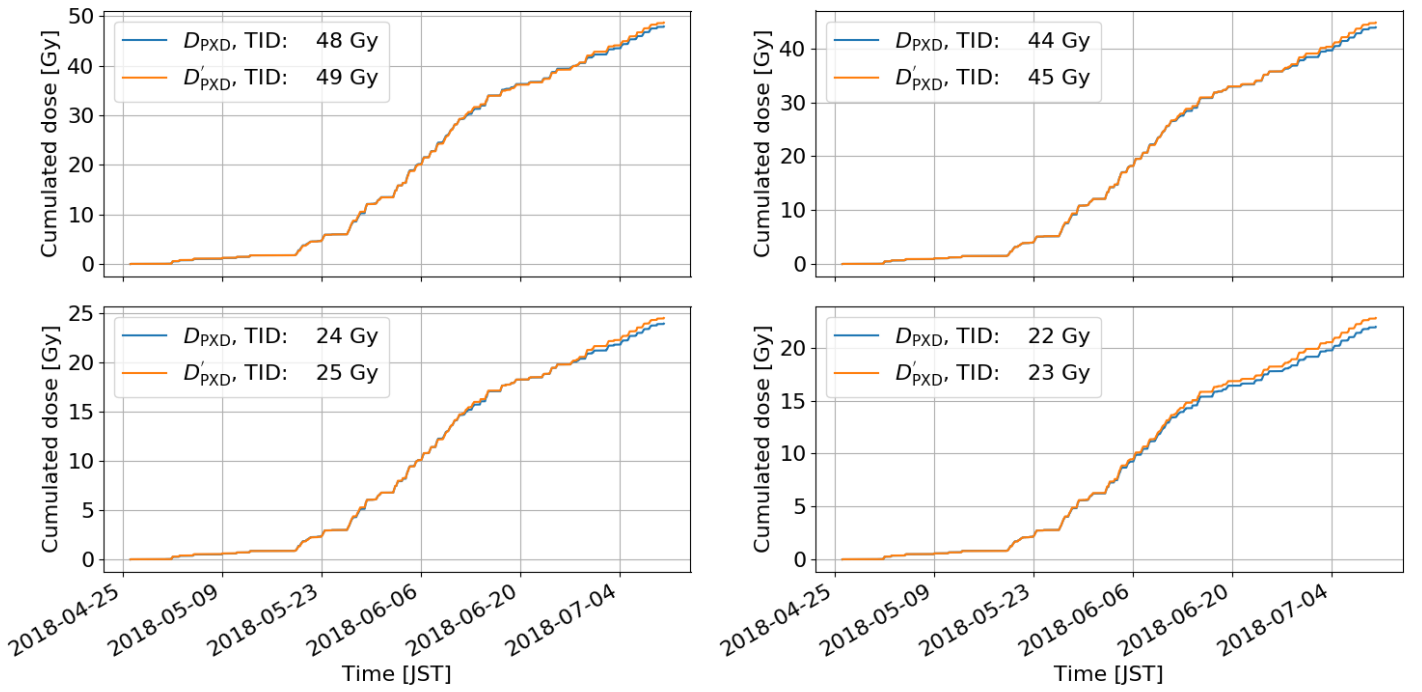

Figure 6.24.: Cumulated sum of the measured dose $D_{\mathrm{PXD}}$ and the calculated dose $D_{\mathrm{PXD}}^{\prime}$. The dataset is restricted to time intervals during which the PXD was on and a dose rate measurement is available. 

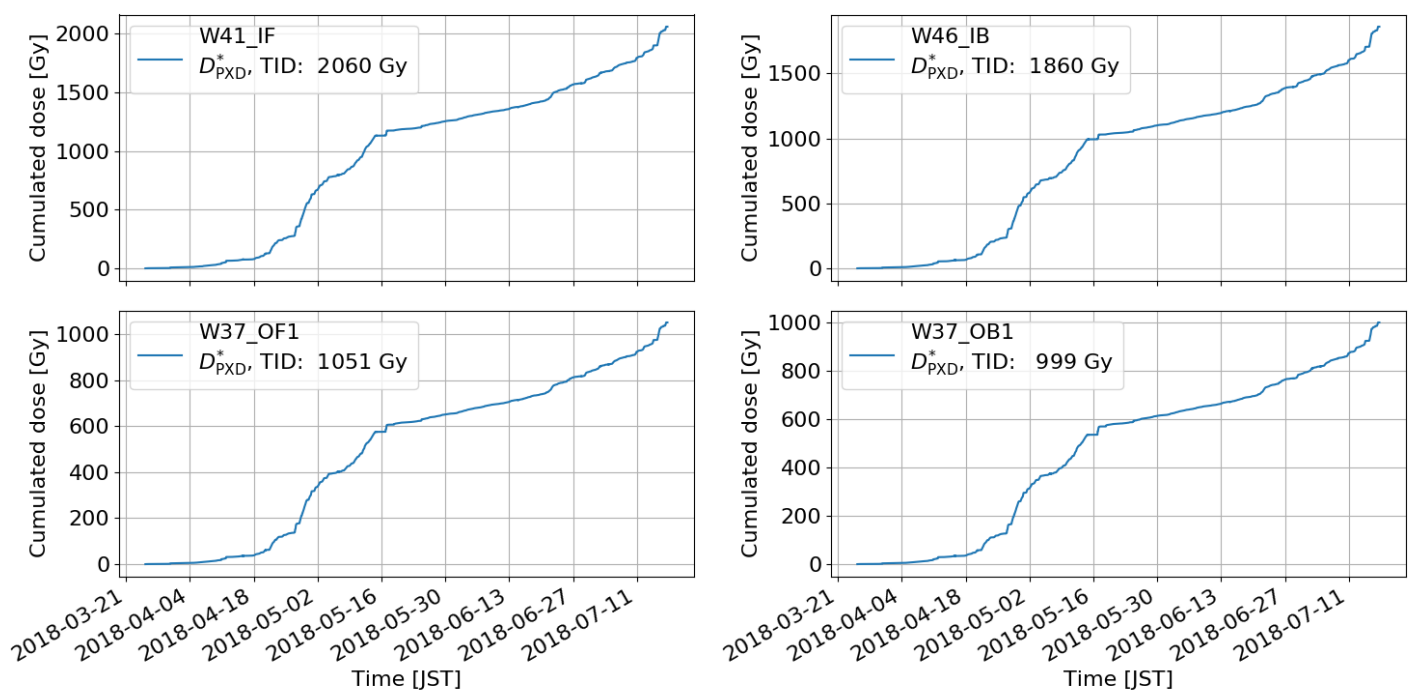

Figure 6.25.: Cumulated sum of the combined dose rates for each PXD sensor for the time between 2018-03-19 and 2018-07-17.

irradiation measurement, where prototype PXD6 DEPFET matrices were used. The comparison shows a significantly better agreement than before with the dose measured purely by the diamond system (see fig. 6.11).

The same analysis strategy was also used to determine the dose received by individual ASIC/Switcher sections on the sensor. Based on the phase 2 VXD geometry, it is possible to create a dose profile along the z-axis of the accelerator as seen in fig. 6.27.

Because nearly $90 \%$ of the dataset that is used to calculate the total dose is not actually measured by the PXD, the values presented here only represent a rough estimation of the ionising dose. The comparison with previous PXD irradiation results validates the results to some degree. Nevertheless, various assumptions are made for the calculation that put a large and difficult to estimate uncertainty on the calculation. 


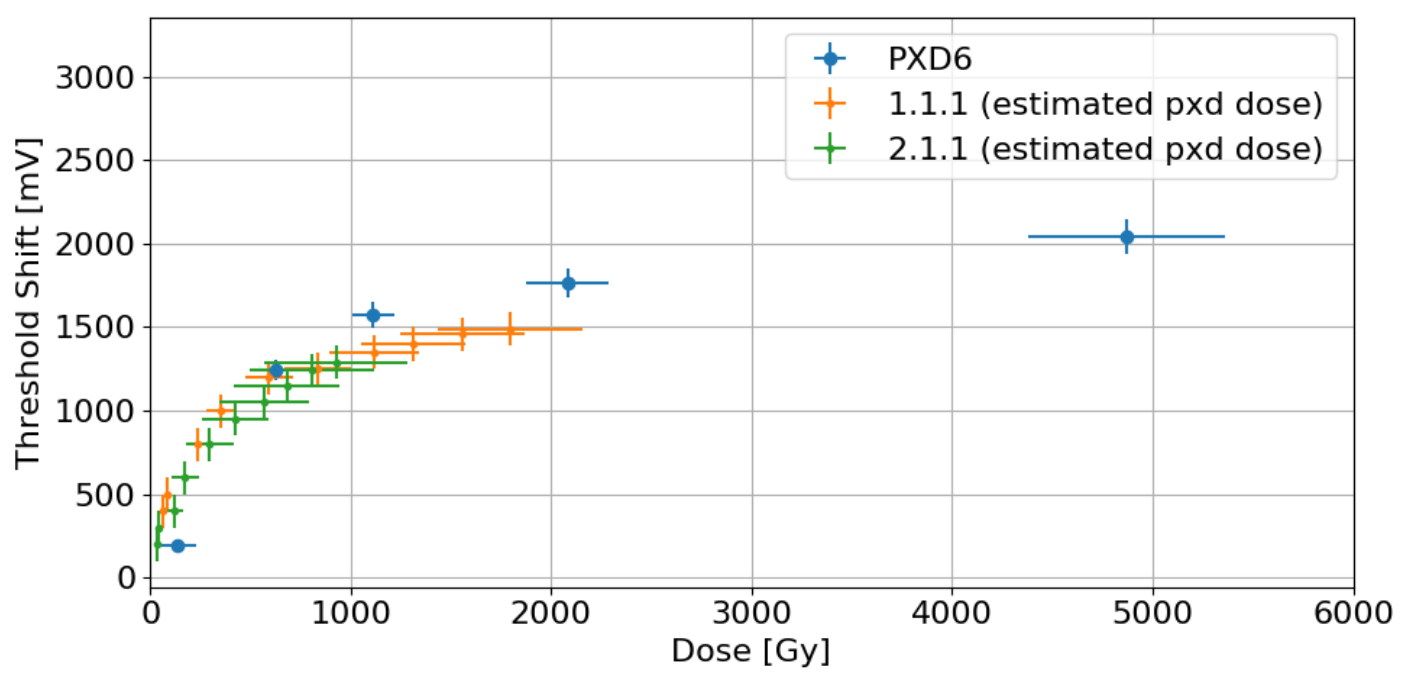

Figure 6.26.: Threshold shift of the phase 2 sensors compared with a previous PXD irradiation measurement. The dose for the phase 2 data points is the calculated dose $D_{\mathrm{PXD}}^{*}$.

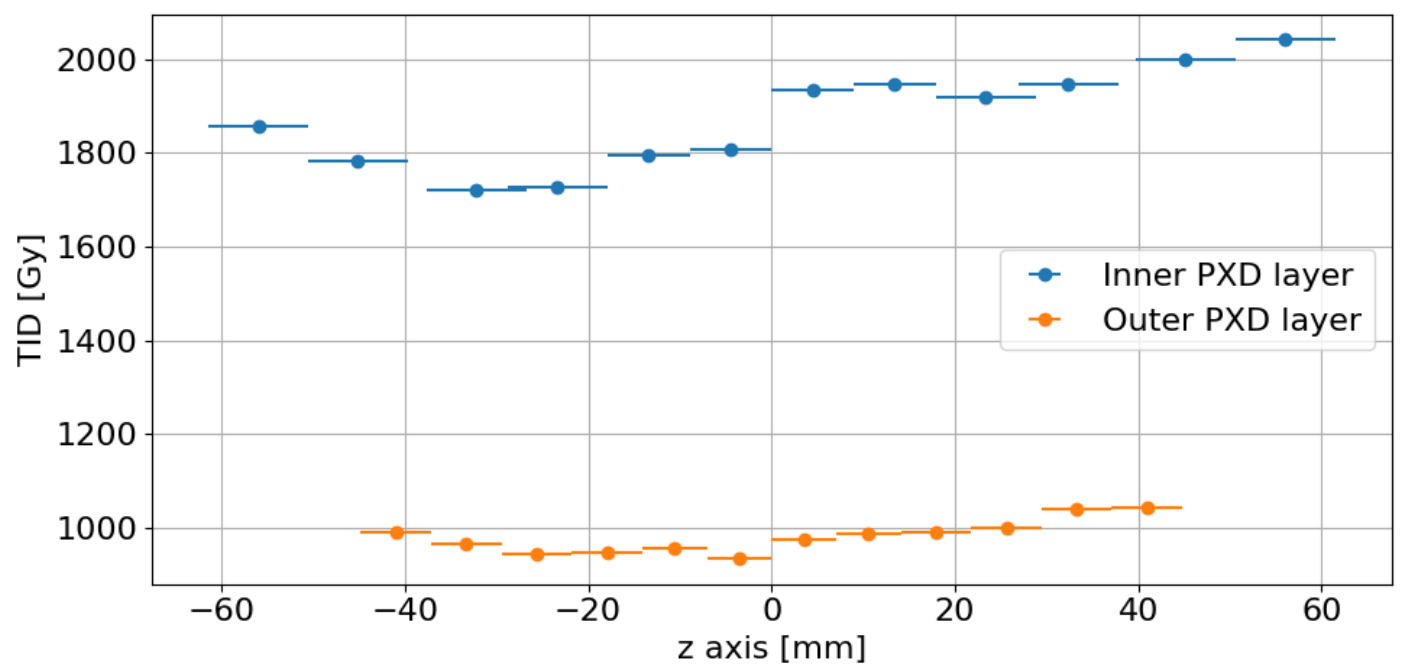

Figure 6.27.: Dose spread along the z-axis. 

CHAPTER 7

Irradiation Experiment

It is essential to understand the effects of ionising radiation on the PXD to guarantee a stable operation under the conditions of Belle II. The experience from phase 2 showed that it is mandatory to re-optimize the operation voltages of the individual PXD half-ladders regularly to ensure a stable performance. During the PXD's development prototype DEPFET structures as well as the ASICs have been irradiated individually but never a complete half-ladder with with final ASICs matching the specification of the half-ladders used in Belle II. For the measurement described in this chapter a half-ladder from the same batch of sensors that are installed in Belle II was used and irradiated up to a dose of $266 \mathrm{kGy}$. The results presented in this chapter have been published in Nuclear Instruments and Methods in Physics Research Section A under the title Effects of gamma irradiation on DEPFET pixel sensors for the Belle II experiment [87].

\subsection{Irradiation Setup}

The irradiation campaign was done at the University of Bonn with a commercial X-ray tube (ISO-DEBYEFLEX 3003, GE Inspection Technologies). The maximum possible acceleration voltage of $40 \mathrm{kV}$ was used and the tube current was set to $50 \mathrm{~mA}$. The tube allows to install various filters directly behind the exit window of the tube to adjust the energy spectrum of the photons. In order to have the highest dose rate, no filter was used for this measurement. Figure 7.1 shows a photograph of the machine. While the machine is operated, the cabinet is closed and the volume is fully darkened. Two devices were used as DUTs (Device under test) during the irradiation, a Hybrid5 (H5026) and a PXD9 outer-backward half-ladder (W05_OB1). 


\section{Irradiation Experiment}

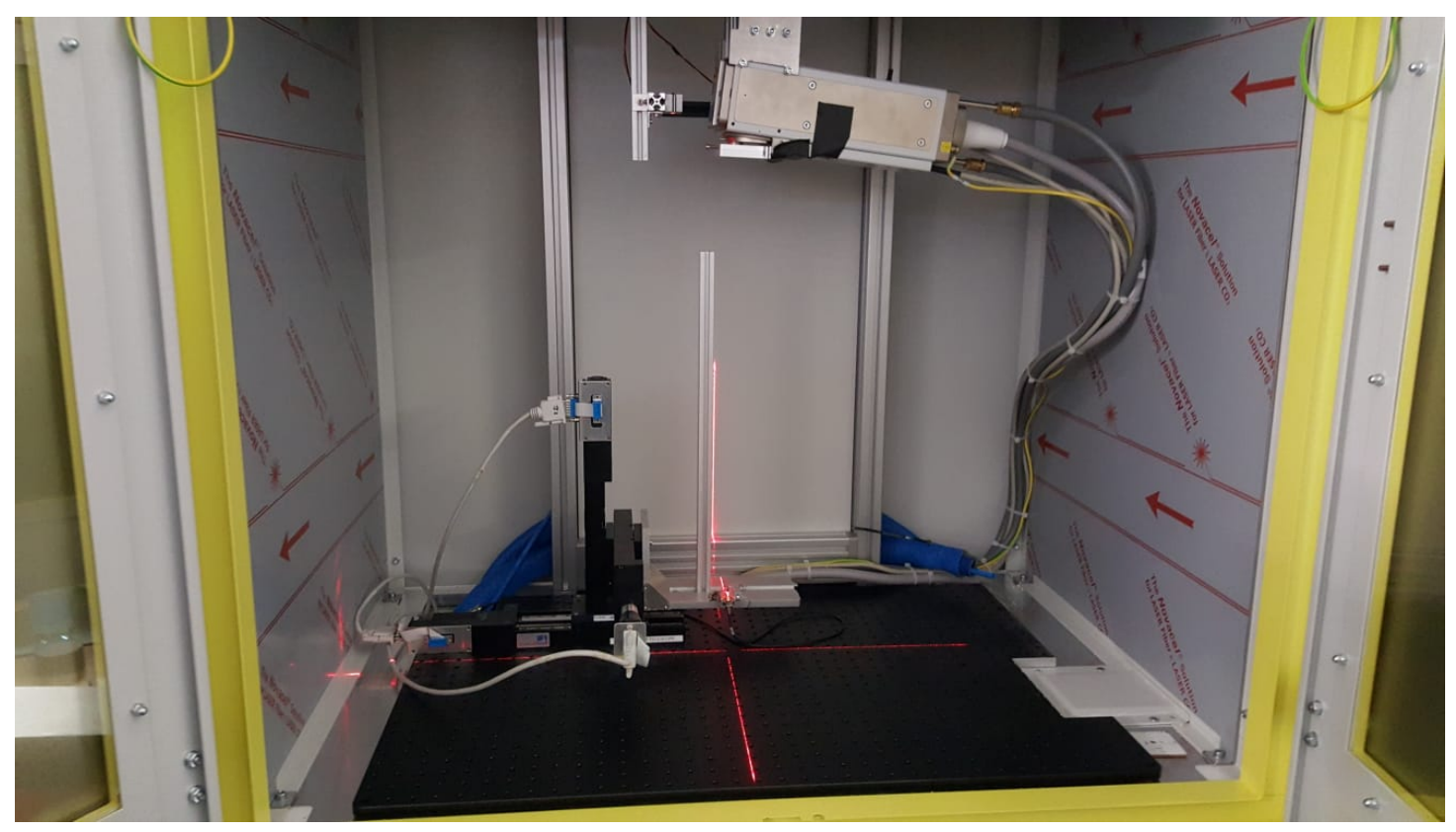

Figure 7.1.: Photo of the X-ray tube used for the irradiation. The tube is placed on the ceiling of the cabinet. A laser cross can be used to align the target device.

\subsubsection{Mechanics and Cooling}

Both devices were put on aluminium cooling blocks and connected to a water-chiller. The motivation was to recreate similar thermal conditions as in Belle II. Studies with a thermal mockup have shown that the expected temperature for the half-ladders is around $35^{\circ} \mathrm{C}$ [36]. The temperatures of the devices were checked using a thermal camera (VARIOSCAN 3021). The camera is cooled with liquid nitrogen during operation and has an absolute accuracy of $\leq 2 \mathrm{~K}$ [88]. To get correct temperature readings though, it is necessary to know the reflectivity of the surface that is looked at. To quantize how strongly the reflectivity of the devices influences the measurement, black paint ${ }^{1}$ was put on the ASICs and the DEPFET matrix of the H5026. Then a thermal image of the device was taken. Figure 7.2 shows a normal image of the Hybrid5 as well as a thermal image. The water-chiller was set to $15^{\circ} \mathrm{C}$. When looking at the DHP in the thermal image, the effect of the black paint is clearly visible. The difference between the surface that is covered with the paint and the surface that is not covered, is $\approx 2.7^{\circ} \mathrm{C}$. For the cooler DEPFET matrix the effect is far less pronounced. Motivated by the results from these images it was decided not to put any paint on the W05_OB1 half-ladder as the measured temperature difference is small enough to get a good estimation of the absolute temperature. Figure 7.3 shows a thermal image of the half-ladder with a chiller temperature of $5{ }^{\circ} \mathrm{C}$. Similar to the Hybrid5, the DCDs are the hottest parts of the sensor. On the DEPFET matrix a temperature gradient is visible, giving temperatures

\footnotetext{
${ }^{1}$ This paint is normally used for the inside of cameras and has a very low reflectivity.
} 


\subsection{Irradiation Setup}

from $\approx 37^{\circ} \mathrm{C}$ near the DCDs to $\approx 31{ }^{\circ} \mathrm{C}$ at the far side of the matrix. The temperatures match those expected at Belle II sufficiently, which is why this chiller setting was used for the irradiation campaign. To make sure that no condensation occurs on the cooling blocks or the devices, they were put into a styrofoam box which was flooded continuously with nitrogen. A picture of the box with the module and the cooling structures can be seen in fig. 7.4.

Only the devices were put into the cabinet of the X-ray machine. The power supplies as well as the readout hardware and computer were located outside next to the machine. For the readout a laboratory readout system as described in section 4.6.3 was used, consisting of a DHE and a computer to record the data. For the powering of the devices a pre-production LMUPS was used.

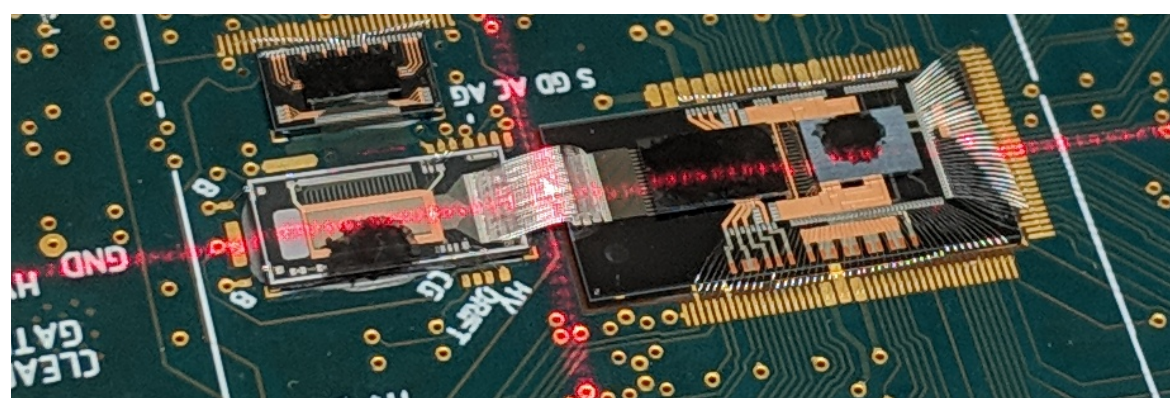

(a)

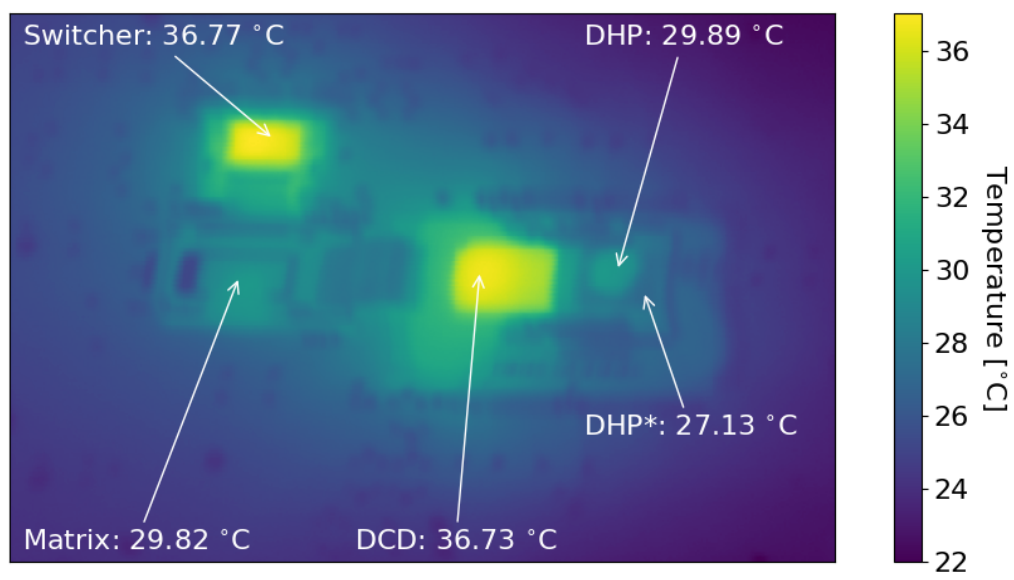

(b)

Figure 7.2.: View of the Hybrid5 device. Figure 7.2a shows a photograph of the matrix and the ASICs. Figure 7.2b shows a thermal image of the same are from a slightly different angle. The DCD and the Switcher are by far the hottest areas. The highlighted spots are covered with black paint except for the DHP* area which was not covered. 


\section{Irradiation Experiment}

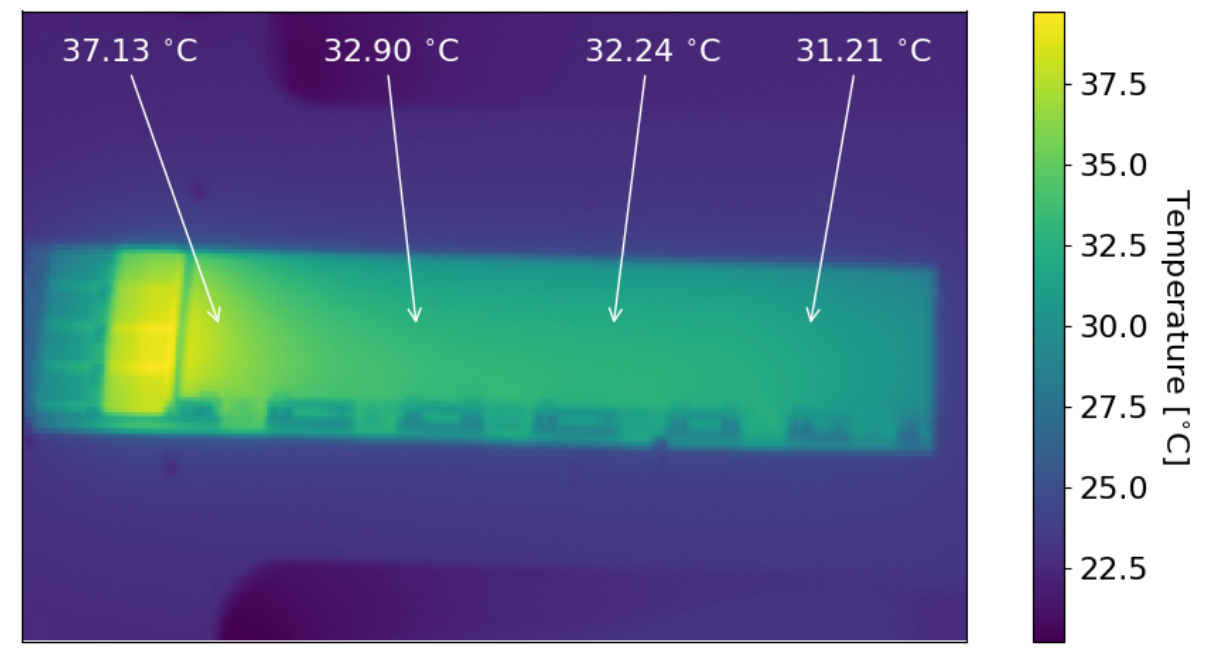

Figure 7.3.: Thermal image of the W05_OB1 with the water chiller temperature set to $5{ }^{\circ} \mathrm{C}$. As the DCDs are biggest heat source, there is a visible temperature gradient along the DEPFET matrix.

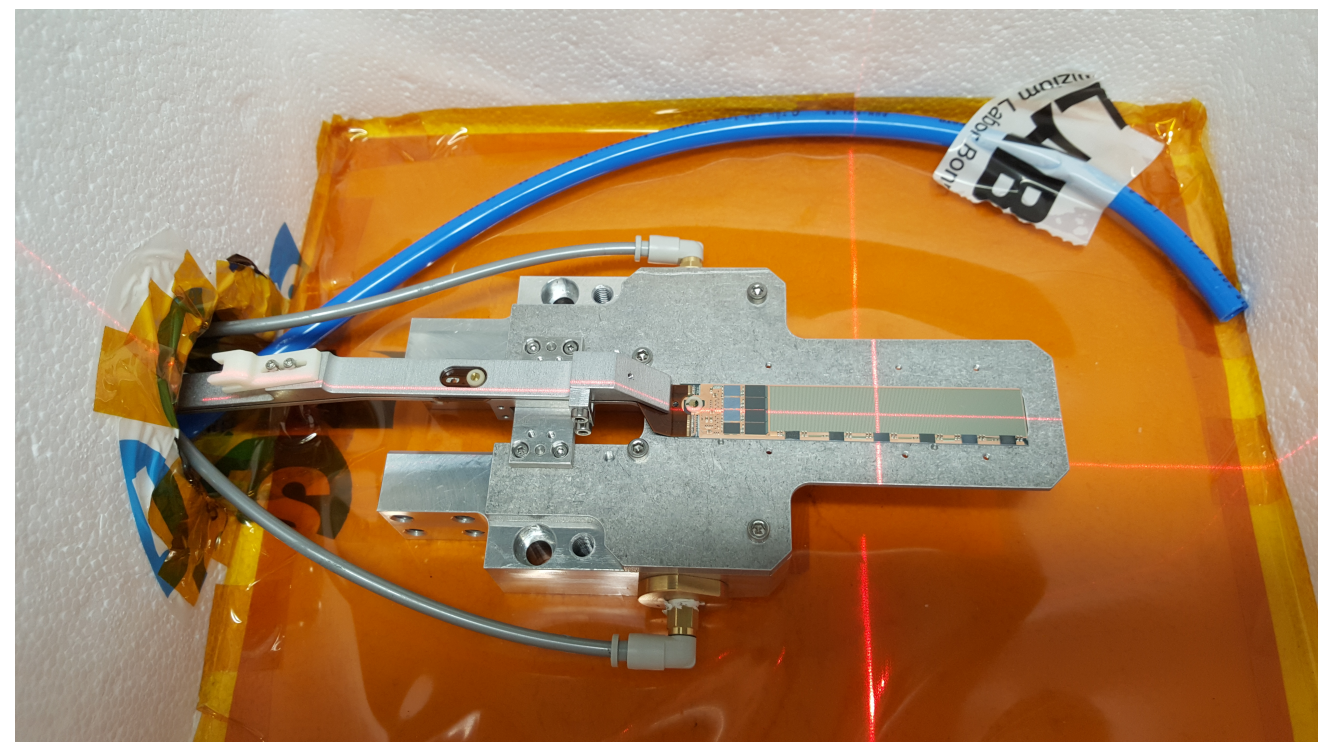

Figure 7.4.: W05_OB1 placed inside a styrofoam box. The aluminium cooling block together with the cooling tubes as well as the tube through which nitrogen is pumped into the volume are visible. 


\subsection{X-ray Spectrum and Geant4 Simulation}

Knowing the energy spectrum of an X-ray tube is an essential part of each irradiation measurement. The energy spectrum is required to calculate the dose deposited in the DUT. The preferred way to get the spectrum is a direct measurement. This is, however, not always possible, especially for X-ray machines with high currents and a high photon production rate. In these cases one can use simulations. The toolkit used most often for these kind of simulations is Geant4 [89]. It simulates the interaction of particles going through matter. In high energy physics Geant4 is used to create simulations of the detector geometry to understand how the particles created in the collisions interact with the detector components. Geant4 is also used in other fields as well like astrophysics and medical physics.

A Geant4 simulation can be broken down in several steps which will be explained in the following.

Geometry Creation The first step of every simulation is the definition of the geometry, in this case the X-ray tube. Each object in the geometry has an associated material. The geometry used in this particular simulation consists of a cube, representing the Tungsten target of the X-ray tube and a cube for the Be window beneath the target. To detect the particles a scoring volume beneath the Be window is defined. The space between the individual objects is modelled as vacuum. A particle gun is used to create the electrons with an energy of $40 \pm 1 \mathrm{keV}$, facing directly to the target. In the simulation the distance between the particle gun and the target is $0.85 \mathrm{~cm}$ and the Be window is placed $0.5 \mathrm{~cm}$ below the target. Figure 7.5 shows a sketch of the used geometry.

Choosing physics process Geant 4 features a variety of physics models that can be used to describe the interactions of high and low energetic particles. The default electromagnetic model used by Geant4 is known to be insufficient to describe low energetic particles, but often sufficient enough for high energy physics. There are, however, alternative models available to improve the simulation in this aspect. For this simulation the Penelope [90] library is used. The lower energy threshold of Geant4, which describes to which energies particles are produced was set to $990 \mathrm{eV}$.

Simulation The last step is the simulation itself. The simulation is split into multiple events. Each events begins with the creation of an electron at the position of the particle gun. The trajectory of the particle is then simulated by Geant4 in discrete steps. When the electron hits the Tungsten target, photons are created which move along their own trajectory. When a photon goes through the Be window and hits the scoring volume, the energy of the photon is registered. By repeating the simulation a few thousand times, an energy spectrum can be simulated. Figure 7.6 shows the result of the simulation. 


\section{Irradiation Experiment}

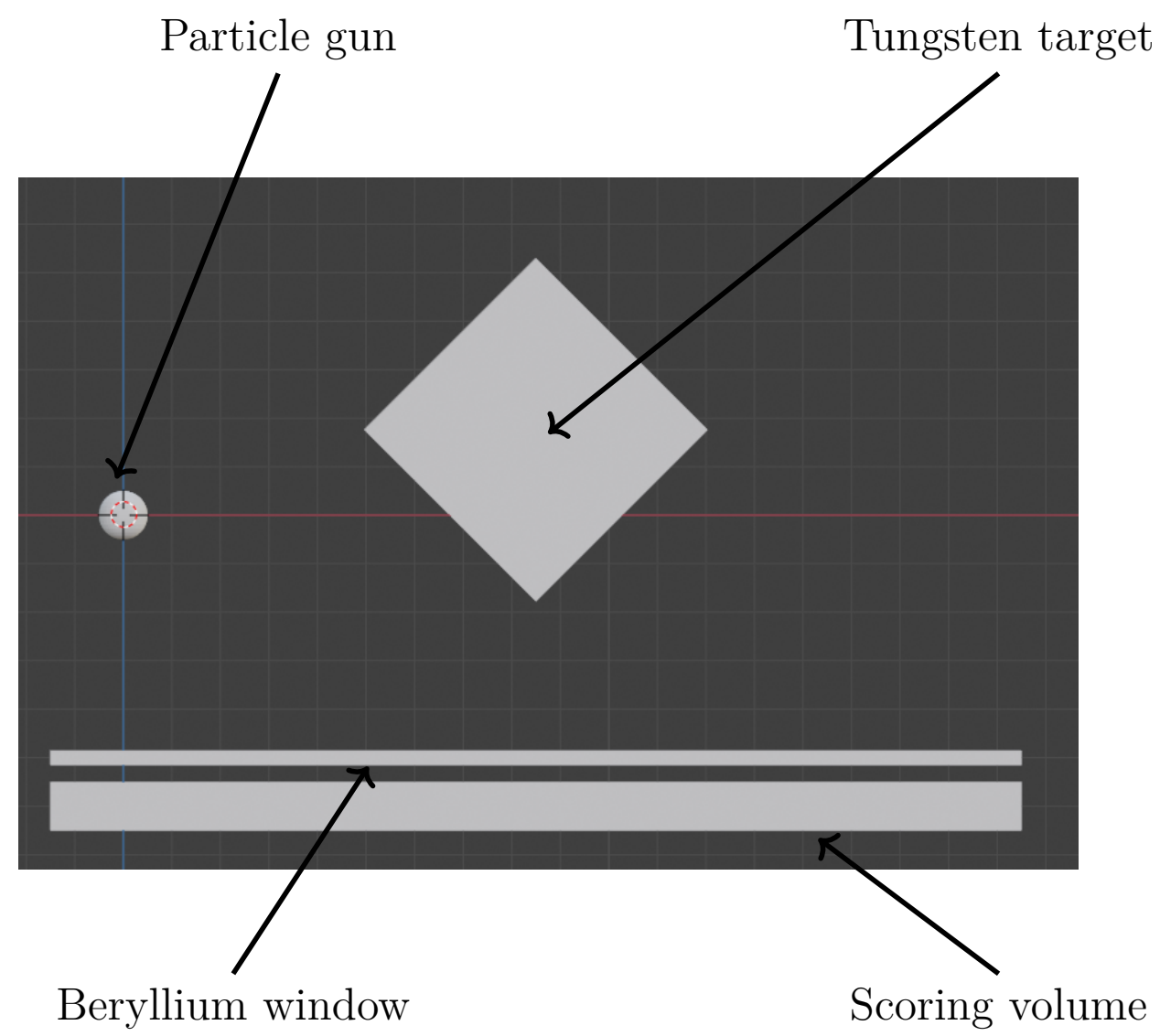

Figure 7.5.: Geometry used for the Geant 4 simulation. The particle gun faces the tungsten directly and the electrons hit the target at an angle of $45^{\circ}$. The created photons go through the Beryllium window volume before they are registered in the virtual scoring volume. 


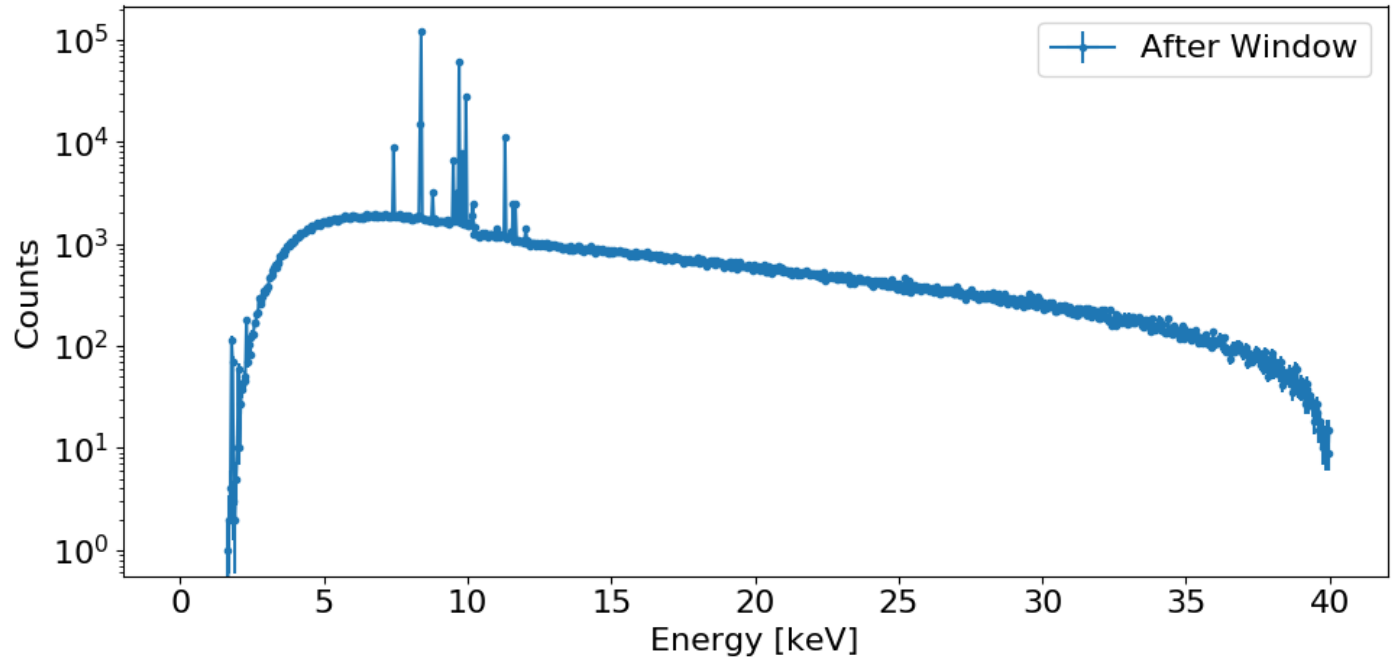

Figure 7.6.: Simulation of the energy spectrum of the X-ray machine. The characteristic lines of tungsten are clearly visible around $10 \mathrm{keV}$ as well as the underlying continuous spectrum. 


\section{Irradiation Experiment}

\subsection{Dosimetry}

A vital part of any irradiation is to understand how much dose is absorbed by the DUT. For this irradiation campaign the most crucial information is how much dose was absorbed in the gate oxides. As outlined in section 4.4, the dose absorbed in the oxide layers of the FET gates creates charges which influence the behaviour of the FETs and introduce a threshold voltage shift.

The physical quantity dose has been introduced before in section 4.4, it is the absorbed energy $E$ per mass $m$ :

$$
D=\frac{E}{m}
$$

To calculate the dose it is necessary to determine the absorption of the photons from the X-ray tube in the DUT. The absorption of $N$ incoming photons in a material is described by the Beer-Lambert law, which can be written as [91]:

$$
N_{\text {abs }}(E)=N(E)\left(1-e^{-\rho \cdot \sigma(E) \cdot d}\right)
$$

Here $N_{\text {abs }}(E)$ is the number of absorbed photons with an energy $E, \rho$ the density of the material, $d$ the thickness of the material and $\sigma(E)$ the energy dependent attentuation coefficient. The absorbed power $P_{\text {abs }}$ in a material can be calculated by integrating over the energy spectrum of the X-ray tube and multiplying it with the production rate $\alpha_{\text {prod }}$ of the tube:

$$
P_{\mathrm{abs}}=\alpha_{\mathrm{prod}} \cdot \int_{0}^{E_{\max }} n(e)\left(1-e^{-\rho \sigma(E) d}\right) \cdot E \cdot \mathrm{d} E
$$

The unknown parameters are the production rate $\alpha_{\text {prod }}$ and the normalised spectrum $n(e)$. It is often difficult to measure the production rate of X-ray tubes that are operated with a high current. There are however ways to circumvent this issue. In the following the method described in [91] will be used to calculate the absorbed dose in the DUTs.

The method consists of two steps. First of all, a reference measurement with a depleted silicon diode is needed. One also needs to know the energy spectrum of the X-ray tube. Reference measurements with diodes were done as part of a general characterization of the machine beforehand. A detailed description of the individual steps and the results can be found in [92].

The reference measurement used in the following calculation was done with a Si diode that had a surface area of $10^{-6} \mathrm{~m}^{2}$ and a thickness of $50 \mu \mathrm{m}$ [93]. The main advantage of a fully depleted Si diode is the near $100 \%$ internal collection efficiency so that each created electron-hole pair contributes to the photocurrent $I_{\text {photo }}$ in the diode:

$$
P_{\mathrm{abssi}_{\mathrm{Si}}}=3.65 \mathrm{eV} \cdot \frac{I_{\text {photo }}}{e}
$$


Where $e$ is the elementary charge and $3.65 \mathrm{eV}$ the energy needed to create one electronhole pair. Based on eq. (7.1), the dose rate of the Si diode is then given as:

$$
\dot{D}_{\mathrm{Si}}=\frac{P_{\mathrm{abs}_{\mathrm{Si}}}}{m_{\mathrm{Si}}}
$$

Here $m_{\mathrm{Si}}$ is the mass of the Si diode. Using eq. (7.4) and eq. (7.5) it is therefore possible to calculate a dose rate from the diode's photocurrent. The diode was used to measure the size and the shape of the beamspot of the machine. Figure 7.7 shows the beamspot at a distance of $60 \mathrm{~cm}$ below the tube together with projections along the $\mathrm{x}$ - and $\mathrm{y}$ axis. The position of the W05_OB1 half ladder is illustrated as well. The measurement shows that there is a significant gradient along the x-axis of the half-ladder, while the dose rate is nearly homogeneous along the y-axis. It is however important to note here

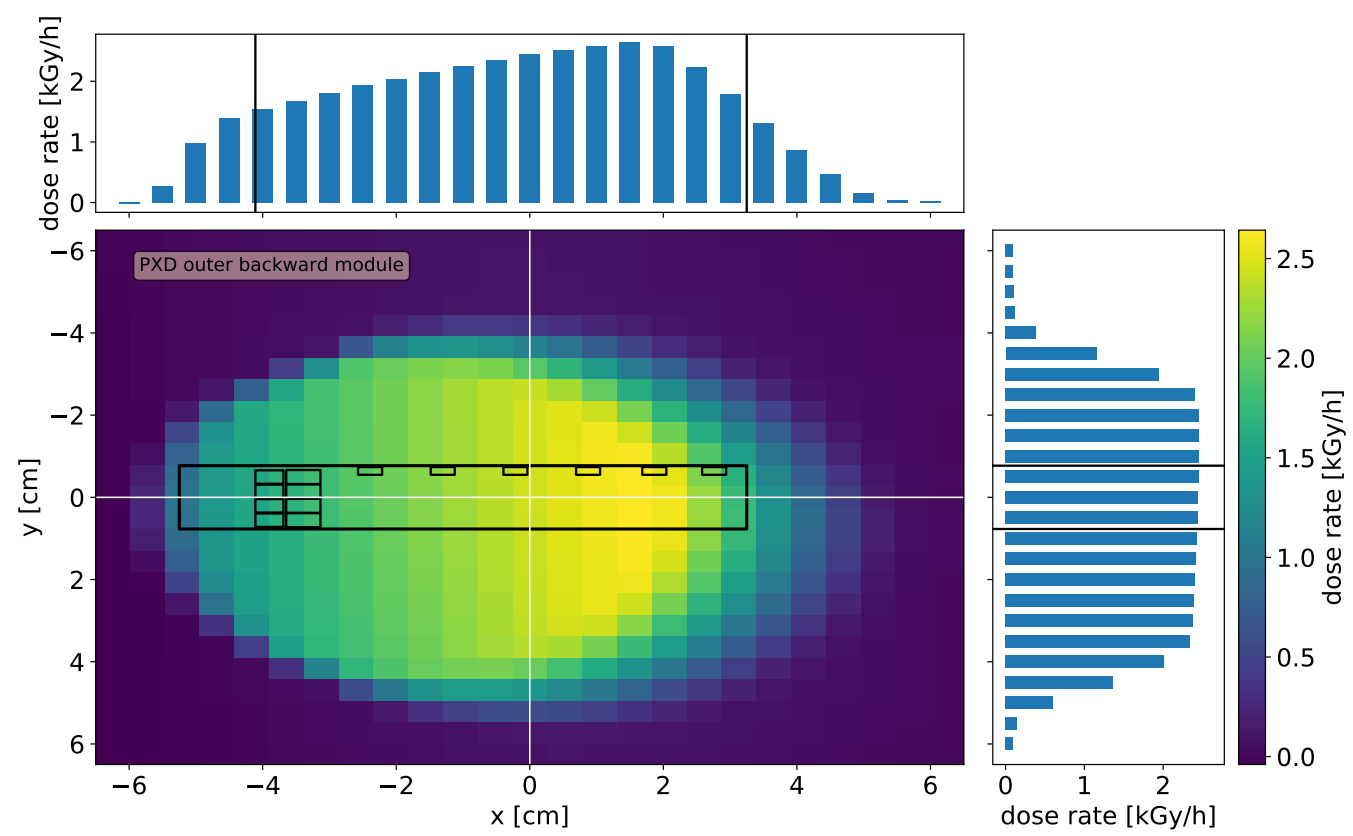

Figure 7.7.: Beam spot of the X-ray tube, measured with a Si diode. The position of the W05_OB1 half-ladder is illustrated as well as size and intensity of the beam spot. Reprinted with permission from [87].

that this measurement is only valid for other silicon devices with the same thickness, as the absorption of the photons depends on the attenuation coefficient and the thickness. Figure 7.8 shows the attenuation coefficients, taken from [94], for $\mathrm{Si}$ and $\mathrm{SiO}_{2}$ in the energy range relevant for this irradiation campaign. While being similar to each other in shape, the absorption in $\mathrm{SiO}_{2}$ is overall slightly lower. By combining eqs. (7.3) and (7.4) the diode measurement can be used to calculate the production rate of the X-ray tube 


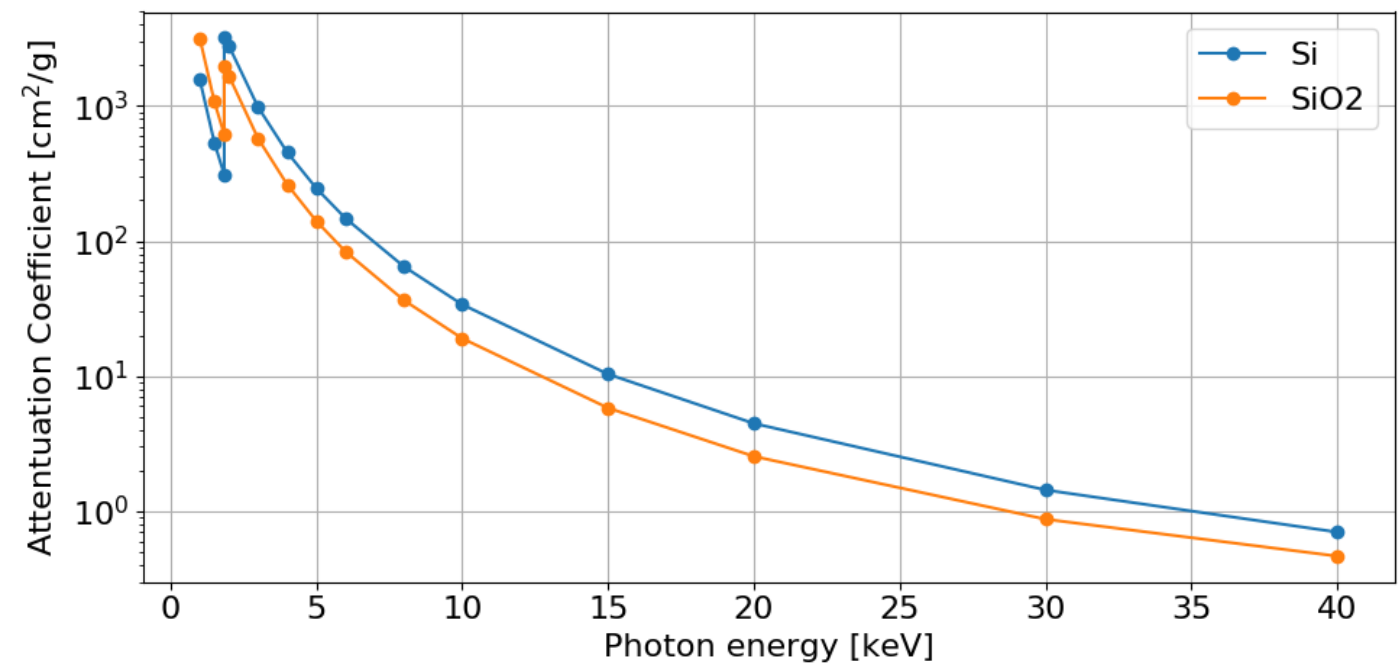

Figure 7.8.: Attenuation coefficient for $\mathrm{Si}$ and $\mathrm{SiO}_{2}$ in the energy range between 1 and $40 \mathrm{keV}$.

as

$$
\alpha_{\text {prod }}=\frac{3.65 e V \cdot \frac{I_{\text {photo }}}{e}}{\int_{0}^{E_{\max }} n(e)\left(1-e^{\left.-\rho_{\mathrm{Si}} \sigma_{\mathrm{Si}}(E) d_{\text {Diode }}\right)} \cdot E \cdot \mathrm{d} E\right.} .
$$

The power absorbed in the gate oxide $\left(\mathrm{SiO}_{2}\right)$ of the DEPFETs with a thickness $d_{\text {gate }}$ is according to eq. (7.3) given as

$$
P_{\mathrm{abs}, \mathrm{SiO} 2}=\alpha_{\mathrm{prod}} \cdot \int_{0}^{E_{\max }} n(e)\left(1-e^{-\rho_{\mathrm{SiO}_{2}} \sigma_{\mathrm{SiO}_{2}}(E) d_{\text {gate }}}\right) \cdot E \cdot \mathrm{d} E .
$$

Combining eqs. (7.6) and (7.7) removes the production rate and yields

$$
P_{\mathrm{abs}, \mathrm{SiO} 2}=P_{\mathrm{abs}, \mathrm{Si}} \cdot \frac{\int_{0}^{E_{\max }} n(e)\left(1-e^{-\rho_{\mathrm{SiO}_{2}} \sigma_{\mathrm{SiO}_{2}}(E) d_{\text {gate }}}\right) \cdot E \cdot \mathrm{d} E}{\int_{0}^{E_{\mathrm{max}}} n(e)\left(1-e^{-\rho_{\mathrm{Si}} \sigma_{\mathrm{Si}}(E) d_{\text {diode }}}\right) \cdot E \cdot \mathrm{d} E} .
$$

If the mass of the gate oxide is known, the dose rate can be calculated by using eq. (7.5) as

$$
\dot{D}_{\mathrm{SiO}_{2}}=\frac{P_{\mathrm{abs}, \mathrm{Si}}}{m_{\mathrm{SiO}_{2}}} \cdot \frac{\int_{0}^{E_{\max }} n(e)\left(1-e^{-\rho_{\mathrm{SiO}_{2}} \sigma_{\mathrm{SiO}_{2}}(E) d_{\text {gate }}}\right) \cdot E \cdot \mathrm{d} E}{\int_{0}^{E_{\max }} n(e)\left(1-e^{-\rho_{\mathrm{Si}} \sigma_{\mathrm{Si}}(E) d_{\text {diode }}}\right) \cdot E \cdot \mathrm{d} E} .
$$

With the simulated X-ray spectrum (see section 7.2) it is possible to scale the dose rate measured with the diode to the one seen by the DEPFET gate oxide. To get a precise estimate of the dose it is important to model the DEPFET correctly. The gate is not 
at the very top of the DEPFET, above it are various metal lines and other structures.

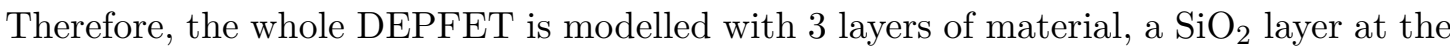
top to model the absorption by the metal lines, a $\mathrm{SiO}_{2}$ layer for the gate oxide and a $\mathrm{Si}$ for the sensitive volume. Table 7.1 summarises the layers, their properties and the expected dose rate based on the diode measurement.

\begin{tabular}{llrr}
\hline & material & density $\left[\mathrm{kg} / \mathrm{m}^{3}\right]$ & dose rate $[\mathrm{krad} / \mathrm{h}]$ \\
layer & & & \\
\hline $\mathbf{1}$ & $\mathrm{SiO}_{2}$ & $2.65 \mathrm{E}+03$ & $1.96 \mathrm{E}+02$ \\
$\mathbf{2}$ & $\mathrm{SiO}_{2}$ & $2.65 \mathrm{E}+03$ & $1.87 \mathrm{E}+02$ \\
$\mathbf{3}$ & $\mathrm{Si}$ & $2.34 \mathrm{E}+03$ & $1.64 \mathrm{E}+02$ \\
\hline
\end{tabular}

Table 7.1.: Dose rates in the individual layers used to describe the DEPFET structure. The structures above the gate are modelled as an additional $\mathrm{SiO}_{2}$ layer.

Because of the gradient in the beamspot of the X-ray tube (see fig. 7.7), each row of the DUT is exposed to a slightly different dose rate, which has to be corrected for when the results of the measurement are analysed. Not only the matrix but the also the ASICs are exposed to the radiation. Because of the flip-chip technique that is used to mount them on the half-ladders, their circuits are shielded from the radiation. The ASICs all have a thickness of roughly $300 \mu \mathrm{m}$. Using this thickness and modelling the circuits with an arbitrary small layer (200 $\mathrm{nm}$ used here), reduces the dose rates significantly. The rates for each ASIC as well as the total dose (TID) a the end of the irradiation can be seen in table 7.2 .

\begin{tabular}{lrrrrrrrr}
\hline & DCD & DHP & \multicolumn{7}{c}{ Switcher } \\
& $1-4$ & $1-4$ & 1 & 2 & 3 & 4 & 5 & 6 \\
\hline Rate [kGy/h] & 0.11 & 0.10 & 0.12 & 0.14 & 0.15 & 0.16 & 0.15 & 0.10 \\
TID [kGy] & 11.88 & 10.88 & 13.26 & 14.69 & 15.92 & 16.85 & 16.39 & 10.75 \\
\hline
\end{tabular}

Table 7.2.: Dose rates and total ionising dose (TID) at the end of the irradiation for all ASICs. The switchers are numbered from the end-of-stave side to the far side of the half-ladder.

\subsubsection{Direct Measurement of the X-ray Spectrum}

The calculation of the dose rates is based on a simulated spectrum. During the irradiation campaign it was tried to record the spectrum of the X-ray tube using the W05_OB1 half-ladder directly, as it has a fast integration time of only $20 \mu \mathrm{s}$. This is however still not fast enough as the following calculation shows. The absorbed power $P_{\text {abs }}$ in the diode can be calculated by integrating over the energy spectrum of the X-ray tube and 


\section{Irradiation Experiment}

multiplying it with the production rate $\alpha_{\text {prod }}$ of the tube:

$$
P_{\mathrm{abs}}=\alpha_{\mathrm{prod}} \cdot \int_{0}^{E_{\max }} n(e)\left(1-e^{-\rho_{S i} \sigma_{S i}(E) d}\right) \cdot E \cdot d E
$$

Here $n(e)$ is the normalised spectrum of the X-ray tube, $\rho_{S i}$ the density, $\sigma_{S i}(E)$ the attenuation coefficient and $d_{\text {Diode }}=5 \cdot 10^{-5} \mathrm{~m}$ the thickness of the diode. The rate $\alpha$ can be calculated when the dose rate $\dot{D}$ is known:

$$
\begin{aligned}
P_{\text {abs }} & =\dot{D} \cdot m_{\text {Diode }} \\
& =0.556 \mathrm{~Gy} / \mathrm{s} \cdot 1.168 \cdot 10^{-7} \mathrm{~kg} \\
& =6.488 \cdot 10^{-8} \mathrm{~W}
\end{aligned}
$$

Using the calculated power it is possible to calculate $\alpha_{\text {prod: }}$ :

$$
\begin{aligned}
\alpha_{\text {prod }} & =\frac{P_{\text {abs }}}{\int_{0}^{E_{\max }} n(e)\left(1-e^{-\rho_{S i} \sigma_{S i}(E) d}\right) \cdot E \cdot d E} \\
& =\frac{6.488 \cdot 10^{-8} \mathrm{~W}}{1.618 \cdot 10^{-33} \mathrm{~J}} \\
& =4.01 \cdot 10^{25} \frac{1}{\mathrm{~s}}
\end{aligned}
$$

Dividing this factor by the area $A=10^{-6} \mathrm{~m}^{2}$ gives the photon flux $\alpha$ :

$$
\begin{aligned}
\alpha & =\frac{\alpha_{\text {prod }}}{A} \\
& =4 \cdot 10^{31} \frac{1}{\mathrm{~s} \cdot \mathrm{m}^{2}}
\end{aligned}
$$

The number of photons absorbed by one gate $\left(A_{\text {gate }}=4 \mathrm{~mm}^{2}\right)$ of the PXD is:

$$
\begin{aligned}
N_{P X D} & =A_{\text {gate }} \cdot \alpha \cdot \int_{0}^{E_{\max }} n(e)\left(1-e^{-\rho_{S i} \sigma_{S i}(E) d_{P X D}}\right) \cdot d E \\
& =212195927 \frac{1}{\mathrm{~s}}
\end{aligned}
$$

During one readout cycle $(20 \mu \mathrm{s})$ this gives $\approx 4200$ Photons in one gate of the PXD. One gate contains 1000 pixels, which gives a mean of more than 4 hits per pixel. If more than one photon hits the same pixel within a readout cycle, it is no longer possible to differentiate the two, which in turn does not allow to record an energy spectrum.

\subsection{Irradiation and Measurement Procedure}

The irradiation of the DUTs was done in steps of varying duration. Before the W05_OB1 half-ladder was irradiated, a pre-experiment with the H5026 Hybrid5 module was performed. After each irradiation step several measurements were performed to quantify 
the performance of the individual components of the sensor, including the DEPFETs and the ASICs. Monitoring of the voltages and currents of the module is done through the LMU power supply. Each step of the irradiation can be split into three phases:

Irradiation: In the first phase the X-ray machine is switched on and the irradiation of the DUT is started. During this phase the DUT is fully biased, powered and ready for data taking. Because of the irradiation the threshold voltage of the DEPFET shifts. This shifts the working point of the transistor and leads to reduced drainsource current. This shift is compensated continuously by adjusting the GateOn voltages (all three Gate-On voltages on the sensor are adjusted by the same absolute values). The target for this compensation is the average drain-source current $\tilde{I}_{D}$ as it is measured by the LMU power supply. A script that adjusts the Gate-On voltage once per second is used to keep the current at $66 \mathrm{~mA}$ at all times. Figure 7.9 shows the evolution of the Gate-On voltage and $\tilde{I}_{D}$ during one of the irradiation phases. During this time full frame raw memory dumps are taken with the sensor in regular time intervals to study changes in the pedestal distribution.

Annealing: Once the X-ray tube is switched off, the annealing phase starts. For the duration of one hour, the DUT is given time to anneal. During this time, the system remains fully powered and the Gate-On voltage is still being adjusted to keep $\tilde{I}_{D}$ constant. In addition memory dumps are taken as in the previous phase.

Measurements: Once the annealing phase is completed, the measurement phase begins. The first measurement performed on the sensor is a scan of the DCD-DHP link parameters. Then IV-curves are taken to determine the threshold shift of the external DEPFET gate. In addition, ADC curves are taken (using the DEPFETs as current source) and the threshold shift of the common clear gate is determined. At the end source scans are done using a ${ }^{90} \mathrm{Sr}$ source $\left(\beta\right.$-radiation) and a ${ }^{109} \mathrm{Cd}$ source ( $\gamma$-radiation).

In total the irradiation consisted of 18 steps. Using the centre of the half-ladder as a reference for the received dose by the DEPFETs, the steps were 0.05, 0.1, 0.25, 0.5, 1 , $2,5,8,11,14,17,20,30,58,100,144,184$ and $216 \mathrm{kGy}$.

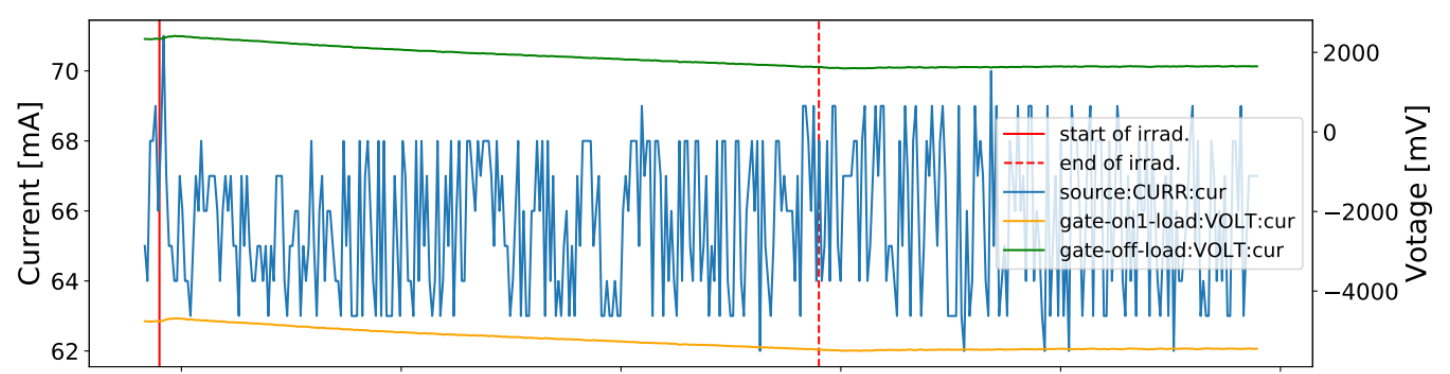

Figure 7.9.: Development of the source current and the Gate-On voltage during the irradiation. 


\section{Irradiation Experiment}

\subsection{Threshold Shifts}

To measure the threshold shift of the external DEPFET gate, I-V curves were recorded by varying the Gate-On voltage $V_{G}$ and recording full frame memory dumps. While this method is not as precise as a measurement of the drain-source current $I_{D}$ with an external device, it has the advantage that IV-curves of all 192000 DEPFET cells are recorded simultaneously.

As the dynamic range of the DCD is limited to $256 \mathrm{ADU}$, the measurement was repeated with different VnSubIn settings on the DCD, which steers the current that is subtracted from the drain-source current inside the DCD (see also section 4.5.3). This effectively "shifts" the I-V curve and allows to record a different part of it. The individual curves (of the same pixel) are later put back together to form a complete I-V curve. Using this approach it is also possible to restrict the analysis to the central part of the dynamic range of the DCD in which the linearity of the ADC is expected to be best [95].

Figure 7.10 shows an example of this I-V curve "stitching" for one pixel. Instead of

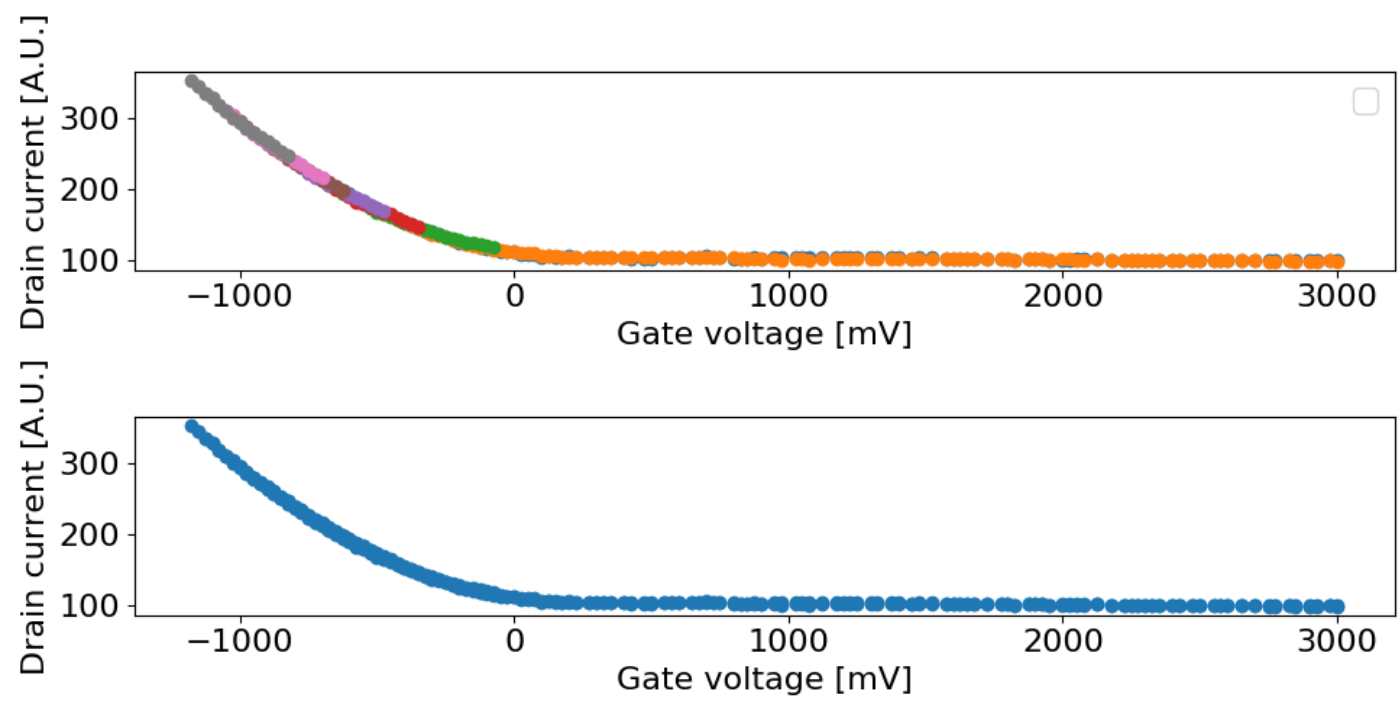

Figure 7.10.: Example I-V curve of one pixel. The different colours in the top figure indicate the VnSubIn setting that was used during the recording of the curve. The bottom figure shows the merged I-V curve.

extracting the absolute threshold voltage from the recorded curves, only the relative shift was calculated by comparing the curves to a reference measurement that was done before the irradiation. For this comparison, which is done for each pixel individually, the curves are binned along the $I_{D}$ axis and the mean gate voltage for each bin is calculated. The binning is chosen such that most bins only contain one or two data points. The mean voltages of each bin are then compared to the corresponding values of the reference measurement and the difference is calculated. The threshold voltage shift is defined as the mean over all bins. 


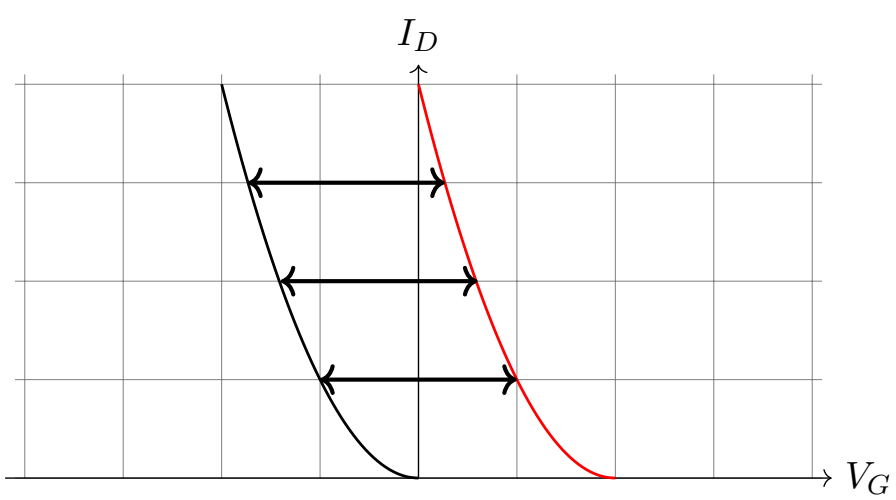

Figure 7.11.: The threshold shift of each irradiation step is calculated by comparing the $\mathrm{I}-\mathrm{V}$ curves before (red) and after (black) the irradiation step. The shift is measured for several bins along the $I_{D}$ axis. Then the average is calculated.

Care has to be taken here not to compare the "flat" part of the I-V curves. A visualisation of this technique on hypothetical I-V curves is shown in fig. 7.11. In fig. 7.12 the evolution of the I-V curves during the irradiation for one pixel is shown.

As mentioned in section 7.3, the gradient of the X-ray beamspot has to be taken into account when the dose for pixel is calculated. A major consequence of this gradient is that one I-V curve measurement is not associated to a single dose value but rather a dose range. This also means that, if the step size is chosen accordingly, two measurements can partly overlap in terms of received dose and a quasi continuous dose distribution is created when the individual measurements are combined. If the step is too large, a gap in the dose continuum occurs.

Figure 7.13 shows a boxplot of the threshold shifts extracted from the I-V curves. The data has been binned along the dose axis and all pixels from all measurements were used to fill the bins. Many bins contain therefore data from different measurements.

The boxplot shows the median as well as the 5\%-95\% interval of the ensemble of threshold shift values for each bin. Nearly all of the dose bins contain at least one data point except for two bins around $50 \mathrm{kGy}$. Here the irradiation step size was too large. A separate inset within the figure shows the low dose range with a finer binning. Here it can be seen that the slope of the threshold shift curves changes. Until $\approx 2 \mathrm{kGy}$ the curve is very steep and then seems to hit a plateau. Around $4 \mathrm{kGy}$ the slope increases again and the curve then follows the expected shape. The maximum threshold shift measured at the end of the irradiation is $\approx 10.5 \mathrm{~V}$ at a dose of $\approx 266 \mathrm{kGy}$.

Not only the external DEPFET gate is affected by the radiation, but also the common clear gate (CCG). Recording an I-V curve is not possible for this gate, because of the PXDs design. It is, however, possible to measure the threshold shift indirectly. When the clear gate voltage is lowered to a certain point, a secondary channel between drain and source of the DEPFET gate is opened. This can be seen directly as an increase 


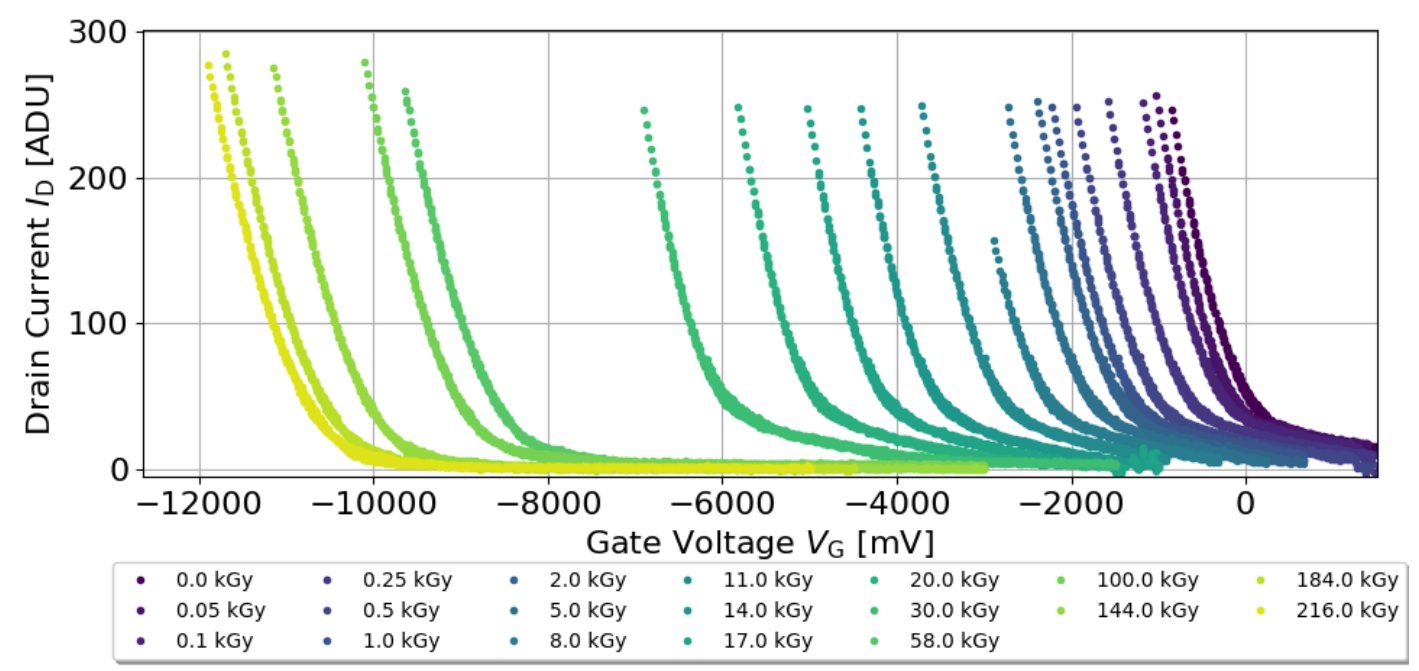

Figure 7.12.: I-V Curves of a single DEPFET pixel at different irradiation steps. Reprinted with permission from [87].

of the source current $\tilde{I}_{D}$ measured by the LMU power supply. Additionally, a small current starts to flow over the drift contact, where under normal operation conditions no current flow is registered by the LMU power suppy ${ }^{2}$. The shift of the threshold voltage is directly correlated to this point which is why it is used to determine the shift. During the measurement, the CCG voltage is lowered in small steps and the drift current is recorded. The reference point is defined by a current of $2 \mathrm{~mA}$ flowing over this line. Similar to the threshold shift calculation of the DEPFET gate, the voltage of this point is compared to a measurement done before the irradiation. The evolution of the shift can be seen in fig. 7.14.

The dose in this plot corresponds to the dose at the central part of the sensor's matrix. A correction for the beam spot is not possible as only a common shift can be determined using the method described above. The shape of the curve is very similar to the one of the DEPFET gate. The major difference is the absolute scale. At the end of the campaign the shift was $\approx 6 \mathrm{~V}$. This is significantly lower (almost 50\%) than the shift seen in the DEPFET gates. As the CCG has a different design and a different oxide than the DEPFET gate, this is to be expected.

Not only the threshold shifts, but also the effects of annealing were studied. Before the DUT was switched off, it remained powered for an additional period of 10 days after the last irradiation step. During this time additional measurements of the DEPFET threshold shift were done and one measurement for the CCG shift. As can be seen in fig. 7.14 , the CCG shift had decreased by $\approx 0.7 \mathrm{~V}$. The DEPFET threshold shift was

\footnotetext{
${ }^{2}$ The resolution of this channel is only $\pm 1 \mathrm{~mA}$
} 


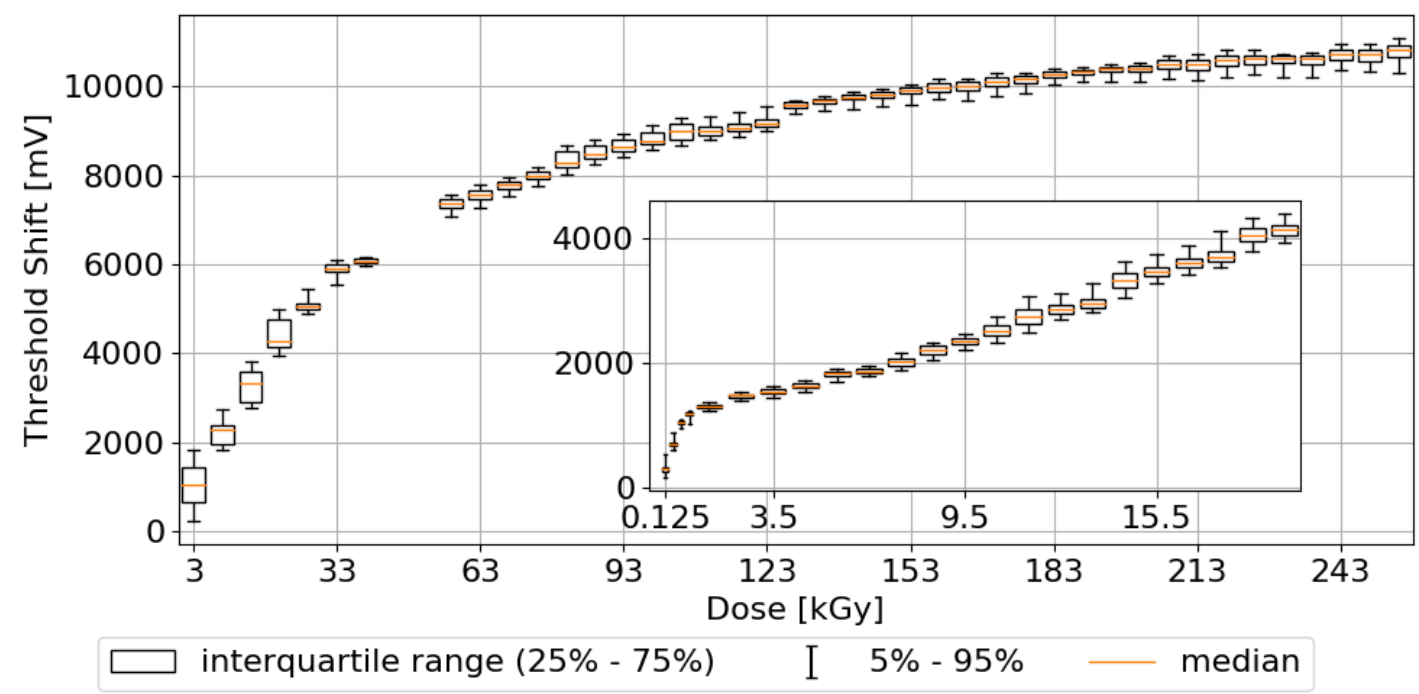

Figure 7.13.: Evolution of the threshold shift during the whole irradiation. Data of all pixels from all measurements is binned based on the TID of the pixel during a measurement. Bin width is $5 \mathrm{kGy}$ for the main figure and $6 \mathrm{kGy}$ for the inset except for the first 4 bins ( $0.5 \mathrm{kGy}$ width). The $\mathrm{x}$-axis shows the mean dose of each bin. Reprinted with permission from [87].

measured six times during this annealing phase. Figure 7.15 shows the evolution of the shift. The figure shows the evolution of three different "regions" defined by their received dose. All pixels that belong to these regions are considered to form ensembles of shifts of which the median is calculated, similar to the process used to visualise the shift during the irradiation. As the figure shows, the trends of all three regions are similar. During the first 100 hours, the shift drops by about $1 \mathrm{~V}$ and then shows only a very slight decrease over the next 140 hours. After $\approx 120$ hours, the temperature of the water chiller was increased from $5^{\circ} \mathrm{C}$ to $10^{\circ} \mathrm{C}$ too check whether the temperature increase affects the annealing. As one can see from the data, this was not the case and no acceleration of the annealing was seen. 


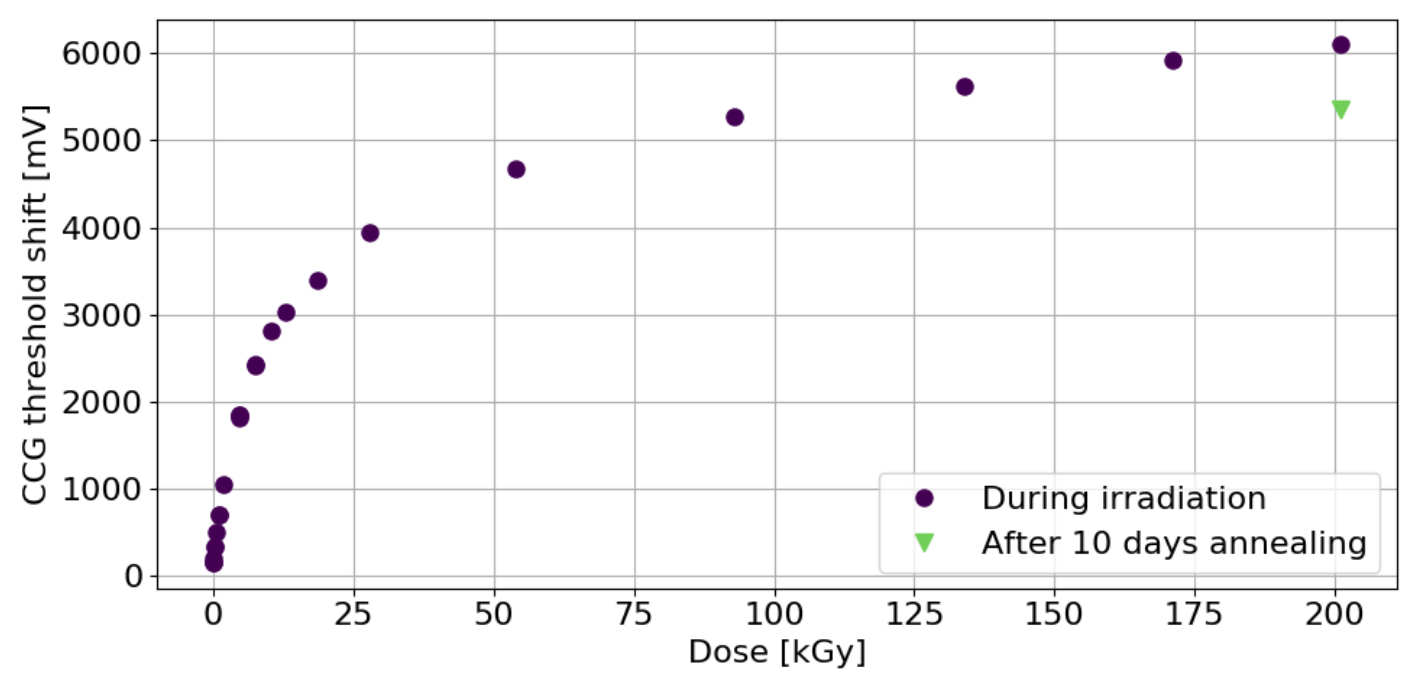

Figure 7.14.: Threshold shift of the common clear gate during the irradiation. Reprinted with permission from [87].

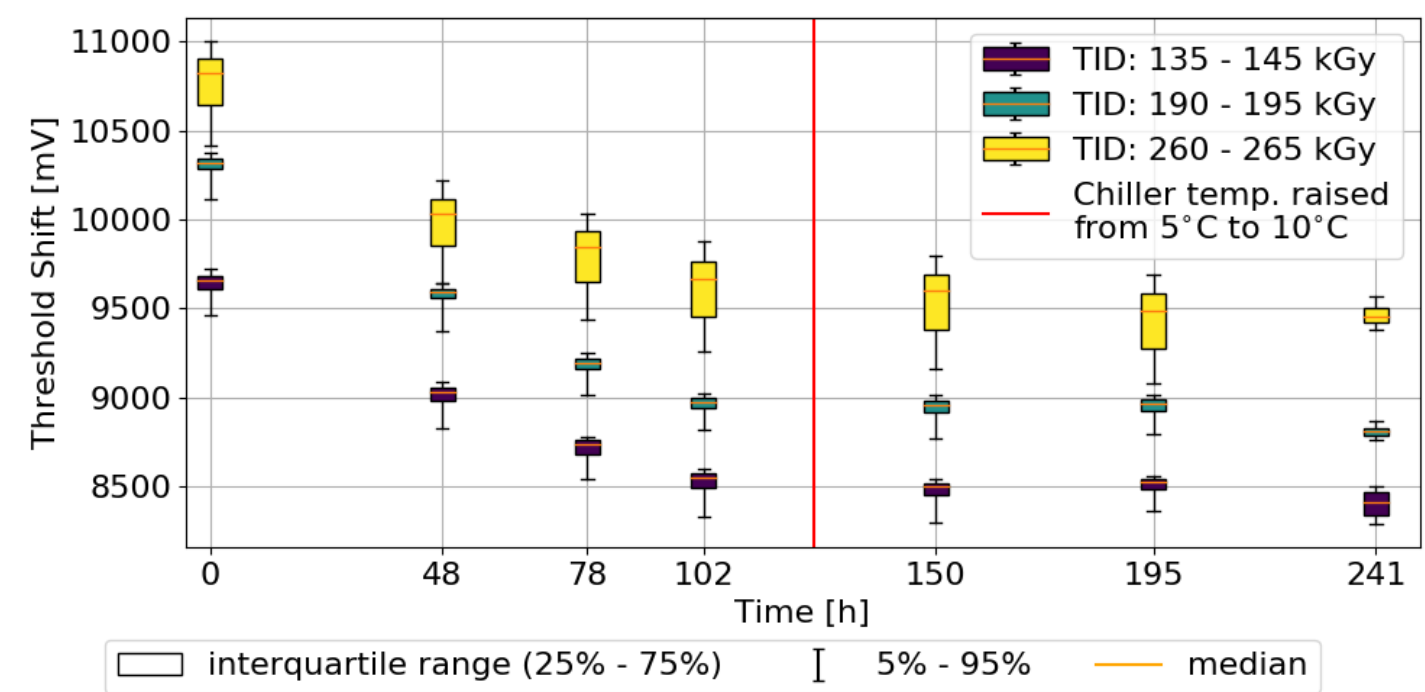

Figure 7.15.: Evolution of the DEPFET threshold voltage shift during the ten day annealing phase at the end of the irradiation campaign. Three different TID regimes are shown. All pixels within the respective regime are taken into account for the calculation of the median and spread. Reprinted with permission from [87]. 


\subsection{Threshold Shift Comparison}

Figure 7.16 shows a comparison of the threshold shift measurement in this irradiation campaign ("PXD9") together with the threshold shift of the phase 2 modules (see section 6.5) and previous irradiation results ("PXD6") [96]. In contrast to the irradiation described here, the PXD6 irradiation used an electron beam and not an X-ray source. Besides, the DEPFET matrix was from an earlier production.

The comparison shows that all datasets agree well with each other. Except for the last datapoint, the PXD6 data shows a slightly higher threshold shift than the PXD9 data. It has to be considered though that the specific conditions of the irradiations differ,

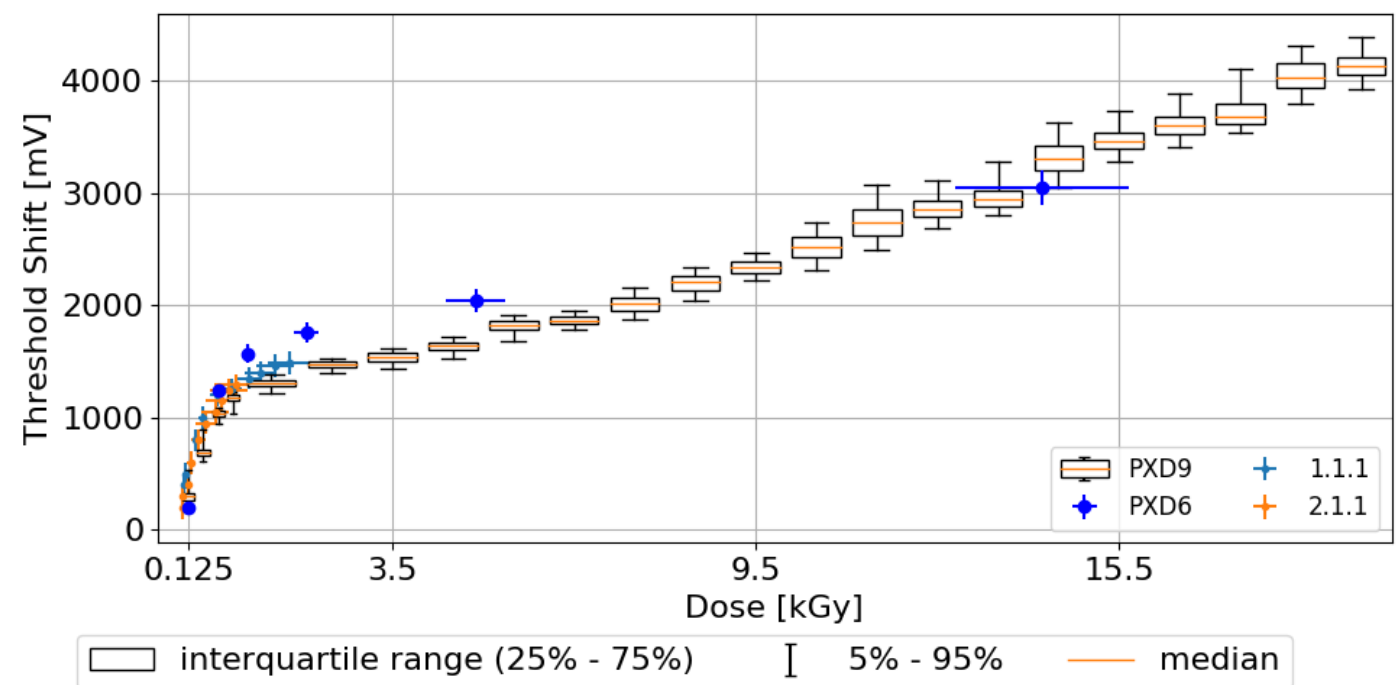

Figure 7.16.: Comparison of the threshold shift measured during the irradiation campaign and previous irradiation measurements. The inset shows data from a previous $e^{-}$irradiation (PXD6) [96]. In addition the voltage shifts calculated in section 6.5 of the inner forward (1.1.1) and the outer forward (2.1.1.) phase 2 half ladders are shown for comparison. Adapted from [87].

especially concerning biasing and annealing. Another key difference is the DEPFET gate length of the two devices: while the PXD9 DUT has a gate length of $5 \mu \mathrm{m}$, the PXD6 DUT had a gate length of $6 \mu \mathrm{m}$. Irradiations with test structures in the past have shown that the observed threshold shift is typically smaller when the gate length is shorter [97].

The threshold shift observed on the phase 2 half-ladders agrees more with PXD9 data. While the phase 2 sensors as well as the DUT of this irradiation are from the same batch of sensors, the conditions of their irradiation differ in key aspect: the rate at which they were irradiated. Because of that, the annealing behaviour and therefore the threshold shift may differ. 


\section{Irradiation Experiment}

\subsection{ASIC Performance}

As mentioned in section 7.3 the dose received by the ASICs was much lower (max. $\leq 20 \mathrm{kGy}$ ) compared to the DEPFETs. Based on previous studies no severe performance degredation is expected. The DCD is expected to have a radiation tolerance of up to $200 \mathrm{kGy}$ [95]. The DHP has been irradiated to a dose of 2 MGy without any degradation in performance [98]. The Switcher ASIC has been irradiated with an X-ray source to a dose of $210 \mathrm{kGy}$ without any observed malfunction [99].

\subsubsection{DHP}

Even with the low dose received in this measurement effects on the ASICs can be seen though. The delay elements of the DHP that are used to adjust the sampling point of the DCD-DHP communication are particularly affected. At the nominal operation voltage of the DHP $(1.2 \mathrm{~V})$, one delay element corresponds to a delay of $355 \pm 20 \mathrm{ps}$ [66]. Because of the irradiation, this delay gets larger, which shifts the optimal sampling point. In total the sampling points for 64 links (for each DCD-DHP pair) have to be optimised. Each link has its own delay setting $\Delta_{\text {local }, i}$. A common global delay $\Delta_{\text {global }}$ is added to each local setting, giving a total delay of $\Delta_{\text {total }, i}=\Delta_{\text {local }, i}+\Delta_{\text {global }}$.

Figure 7.17 shows two examples of a phase space scan for one of the links. The first one was recorded before the irradiation had started, the other one directly after. The region of good delay settings is visibly shifted to the left (smaller delay). If this shift gets too large it is no longer possible to find a working delay setting. To quantify the degradation of inter-chip communication performance, the number of working settings is counted. Due to the shift this number is slowly decreased. Figure 7.18 shows this quantity as function of received dose. Here the numbers are normalised to their initial values and only the relative decrease is shown. For doses below $10 \mathrm{kGy}$ no degradation is seen. For higher doses the number of error-free settings decreases but never falls below $80 \%$.

From the recorded data it is also possible to calculate the length of one delay element at each step of the irradiation. To do this, the shift of the "band" of error-free settings is measured. Together with the knowledge of the delay length at the beginning, this allows to calculate the delay length at any point. Figure 7.19 shows the delay length as a function of received dose. At the end of the irradiation, the delay per element was $393 \pm 21$ ps. This corresponds to an increase of $11 \%$. 

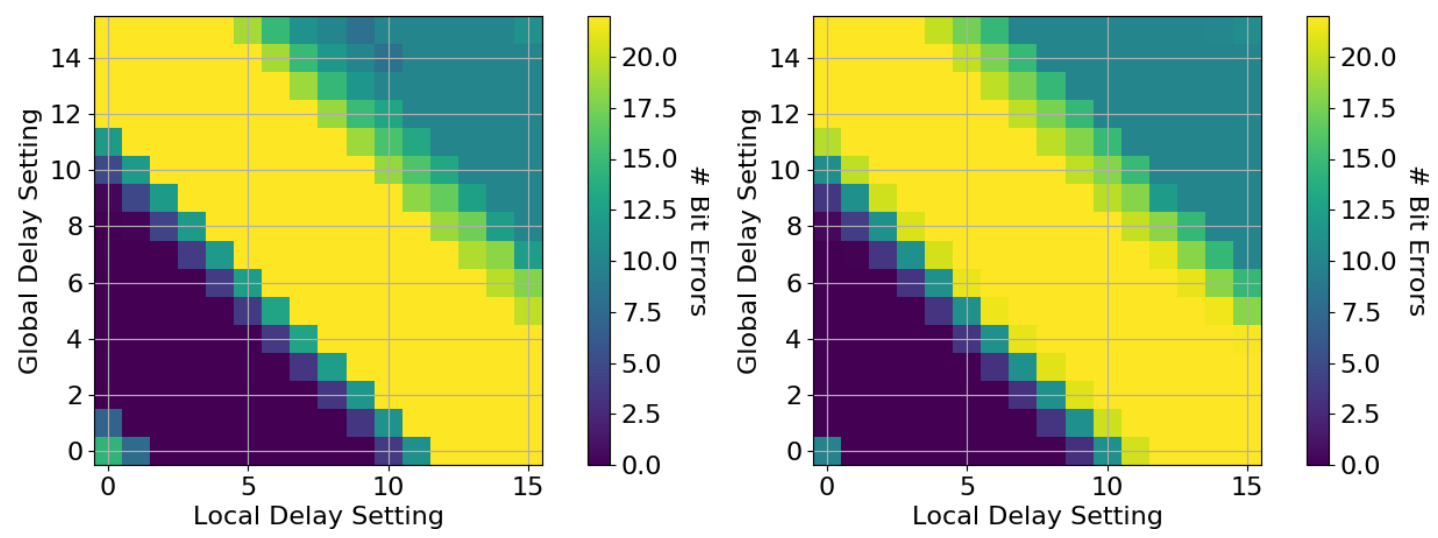

Figure 7.17.: Delay optimisation scan before (left) and after (right) the irradiation for a single link. The x-axis shows the number of local delay elements put in series and the y-axis the number of global delay elements. The colour shows the average number of bit errors observed in 100 data frames. The blue band showing the error-free region in the parameter space shows a clear shift.

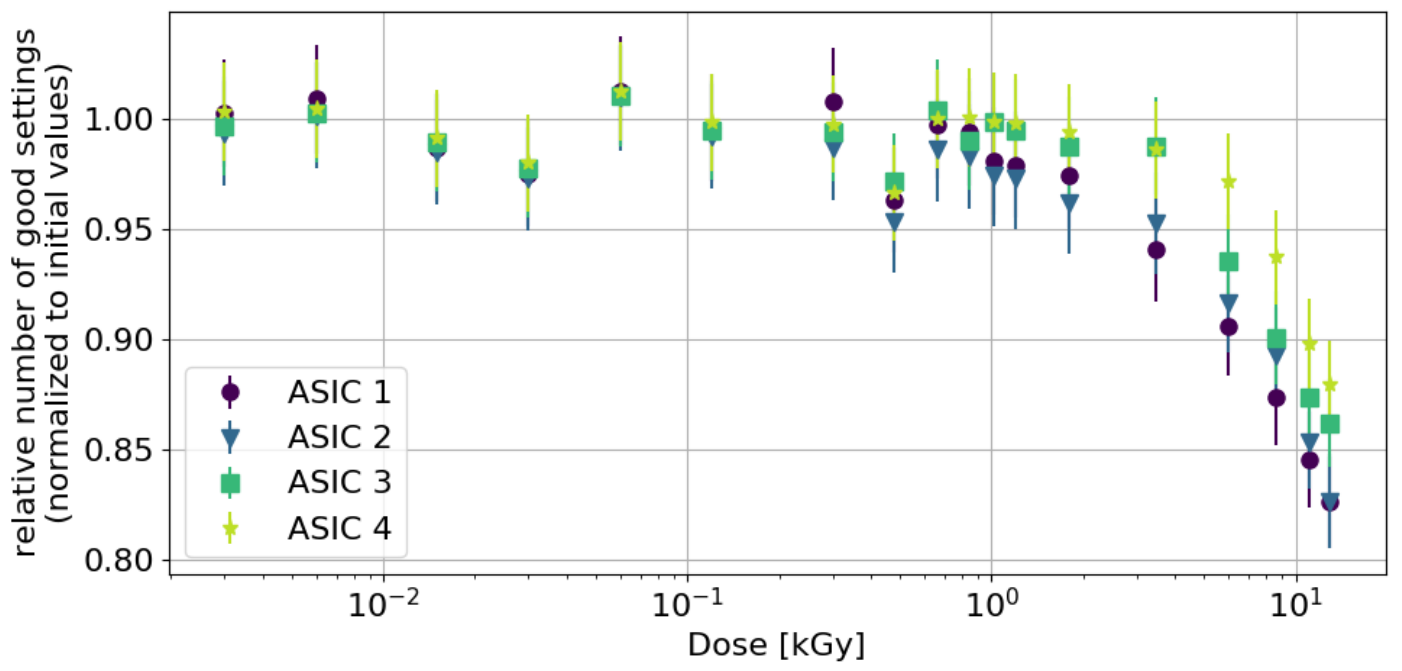

Figure 7.18.: Relative number of error-free delay settings for the DHP-DCD communication. The errorbars correspond to the statistical poisson error. Reprinted with permission from [87]. 


\section{Irradiation Experiment}

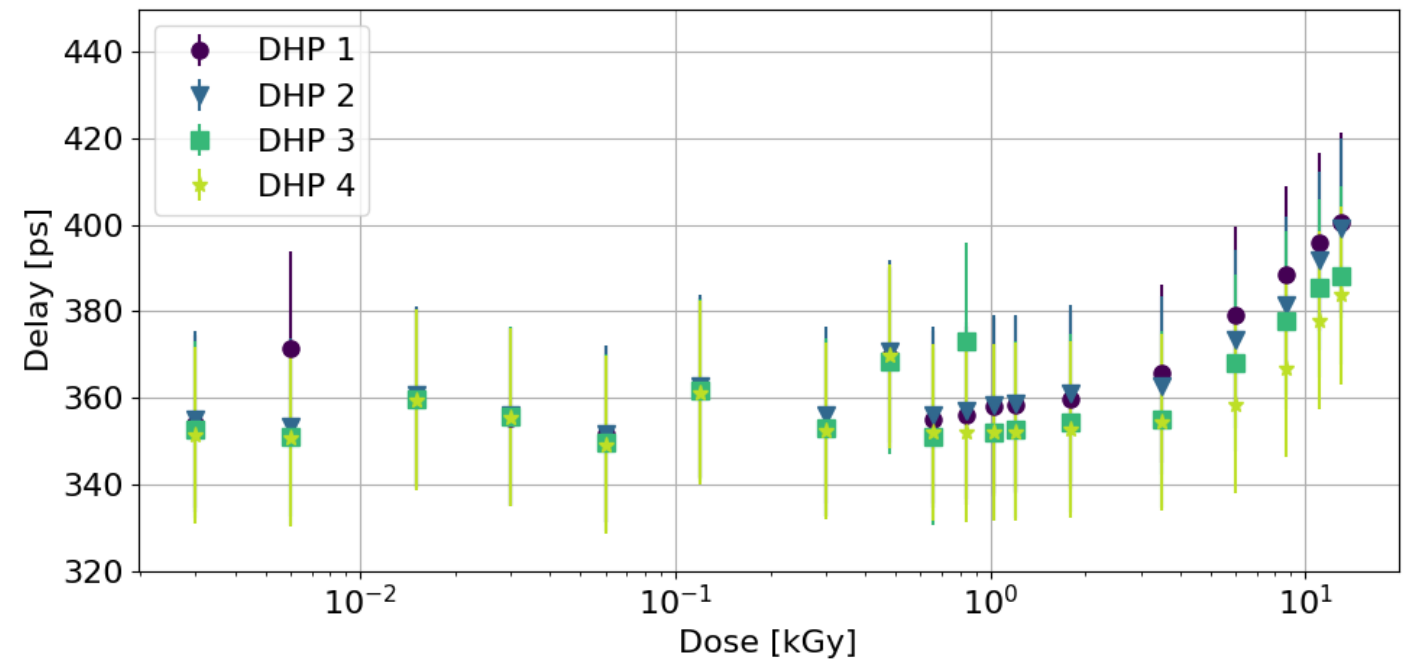

Figure 7.19.: Evolution of the delay length of a single delay element, averaged over all links of one DHP. Reprinted with permission from [87]. 


\subsubsection{DCD}

As described in section 4.5.3 and appendix A, the performance of the DCD can be judged best by looking at ADC transfer curves. Ideally, a scan over the complete phase space of DCD parameters would have been done. Due to time constraints this was not possible. A scan over the voltages $V_{\text {Amplow }}$ and $V_{\text {RefIn }}$ was done once during the irradiation at a dose of $1 \mathrm{kGy}$.

A comparison with the same phase space scan done before the irradiation can be seen in fig. 7.20. The figure shows the how many DCD channels pass the individual quality criteria for each setting of $V_{\text {Amplow }}$ and $V_{\text {RefIn }}$. Especially in the product of all quality criteria, a difference between the two measurements is visible. Looking at the individual criteria, the biggest shift can be seen in the code-range and long codes numbers.

At the other steps of the irradiation no parameter scan was performed and transfer curves were only recorded for the pre-irradiation working point. The number of gradeA/B/F channels can be used to classify the performance at this working point. Table 7.3 summarises these number at the start and at the end of the irradiation. No significance of performance is seen at the final dose of $12 \mathrm{kGy}$.

\begin{tabular}{c|c|c|c}
\hline Dose [kGy] & Grade-A channels & Grade-B channels & Grade-F channels \\
\hline $\mathbf{0}$ & 644 & 322 & 34 \\
$\mathbf{1 2}$ & 620 & 342 & 38 \\
\hline
\end{tabular}

Table 7.3.: Comparison of the DCD performance before and after the irradiation. The 1024 DCD channels are divided into three categories based on criteria like noise, linearity and dynamic range. Reprinted with permission from [87]. 


\section{Irradiation Experiment}

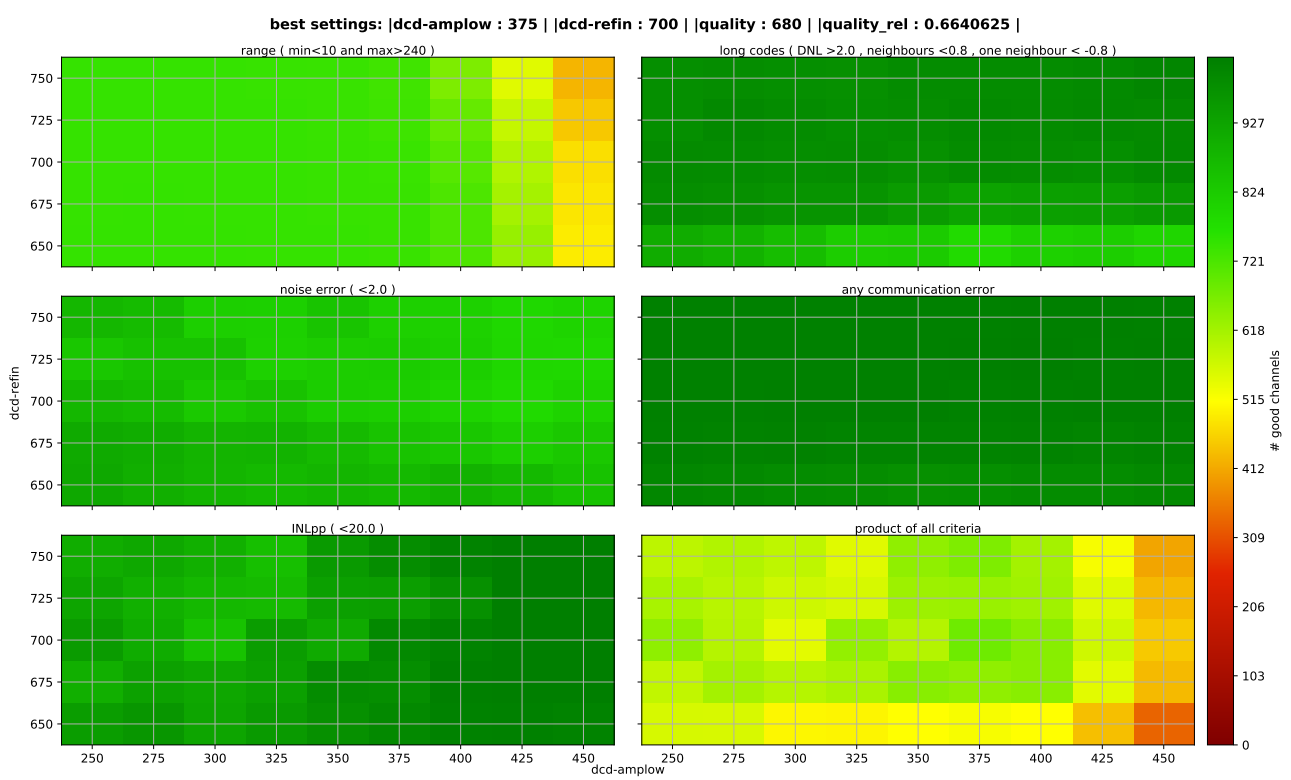

(a) $0 \mathrm{kGy}$

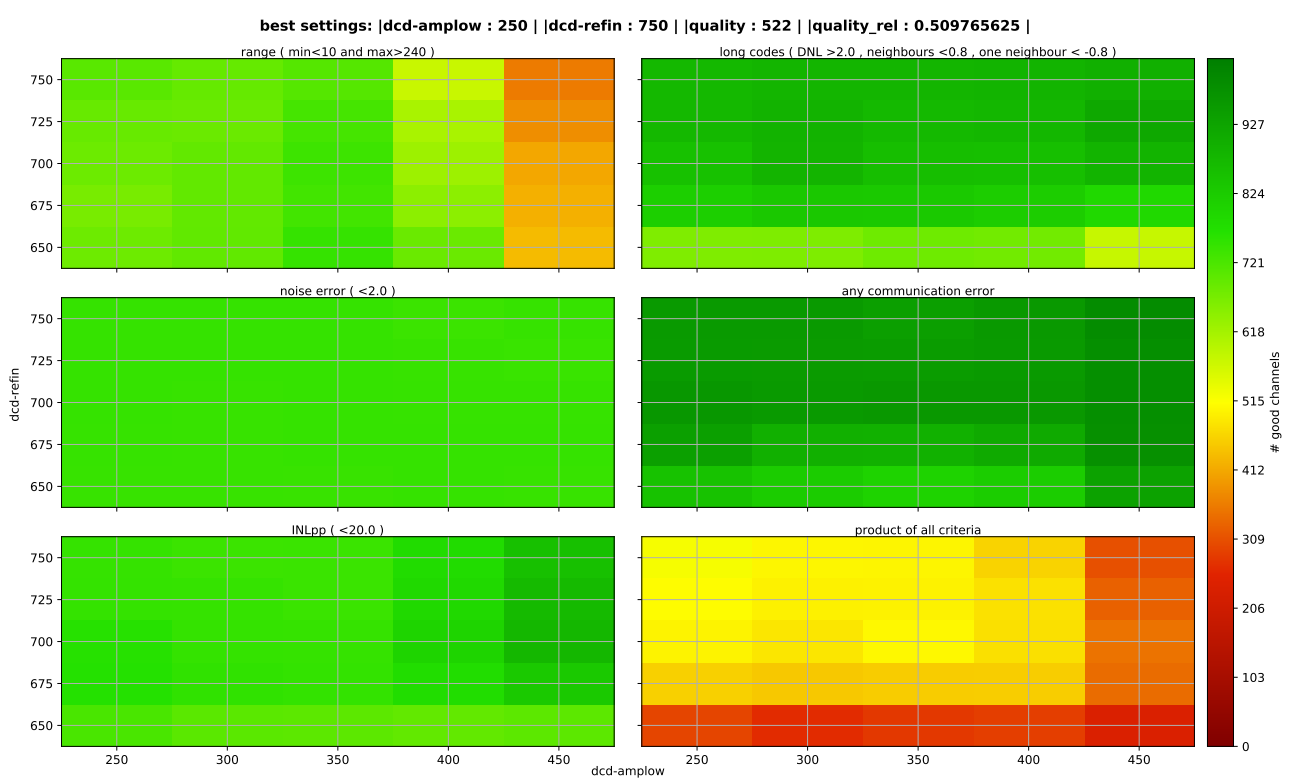

(b) $17 \mathrm{kGy}$

Figure 7.20.: Scan over Amplow-RefIn voltages before the irradiation and during the irradiation at a dose of $1 \mathrm{kGy}$. 


\subsection{Radioactive Source Measurements}

To study the performance of the overall system, measurements with radioactive sources were done as the last measurement before the X-ray tube was activated again. These measurements allow to check the working point of the DEPFETs by looking at the MPV of the cluster charge distributions. In addition it it possible to determine the signal-tonoise ratio. Table 7.4 lists the radioactive sources used during the irradiation campaign together with their radiation type and energies. For these measurements the sensor was

\begin{tabular}{lll} 
Isotope & Radiation Type & Energy $[\mathrm{keV}]$ \\
\hline${ }^{90} \mathrm{Sr}$ & $\beta$ & $546(\max )$. \\
${ }^{109} \mathrm{Cd}$ & $\gamma$ & 22.16 \\
\hline
\end{tabular}

Table 7.4.: Radioactive Sources used during the irradiation.

not removed from the X-ray machine cabinet. The radioactive sources were placed a couple of centimetres above the sensor in a central position. For the data taking no external trigger was used. Instead the sensor was continuously read out at a frequency of $10 \mathrm{kHz}$. A hitmap of a measurement with a ${ }^{90} \mathrm{Sr}$ source can be seen in fig. 7.21 One

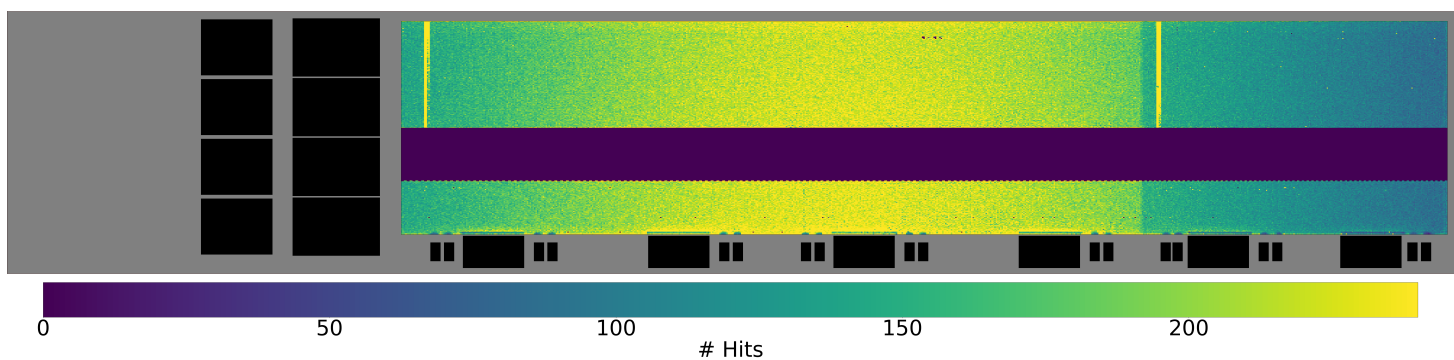

Figure 7.21.: Hitmap of the PXD after a ${ }^{90} \mathrm{Sr}$ measurement at the beginning of the irradiation. The beam-spot of the source is clearly visible and the sensor as well as the deactivated ASIC pair. The two yellow lines correspond to two damaged (electrical) gates of the sensor.

of the visible features are the two segments along the $\mathrm{x}$-axis with different pixel pitches. At the bottom side of the figure one can also identify the positions of the switchers and additional capacities that seem to at least partly shield the DEPFET matrix from the radiation. The third DCD-DHP was not read out during all measurements because a high noise was seen that made a stable readout impossible due to a too high occupancy ${ }^{3}$. Using the same clustering strategy as described in section 6.4, the recorded data was processed and analysed.

Figure 7.22 shows two cluster charge distributions recorded with the sensor at the beginning and at the end of the irradiation. Both distributions show the expected

\footnotetext{
${ }^{3}$ Each DHP can only handle an occupancy of $\approx 3 \%$
} 


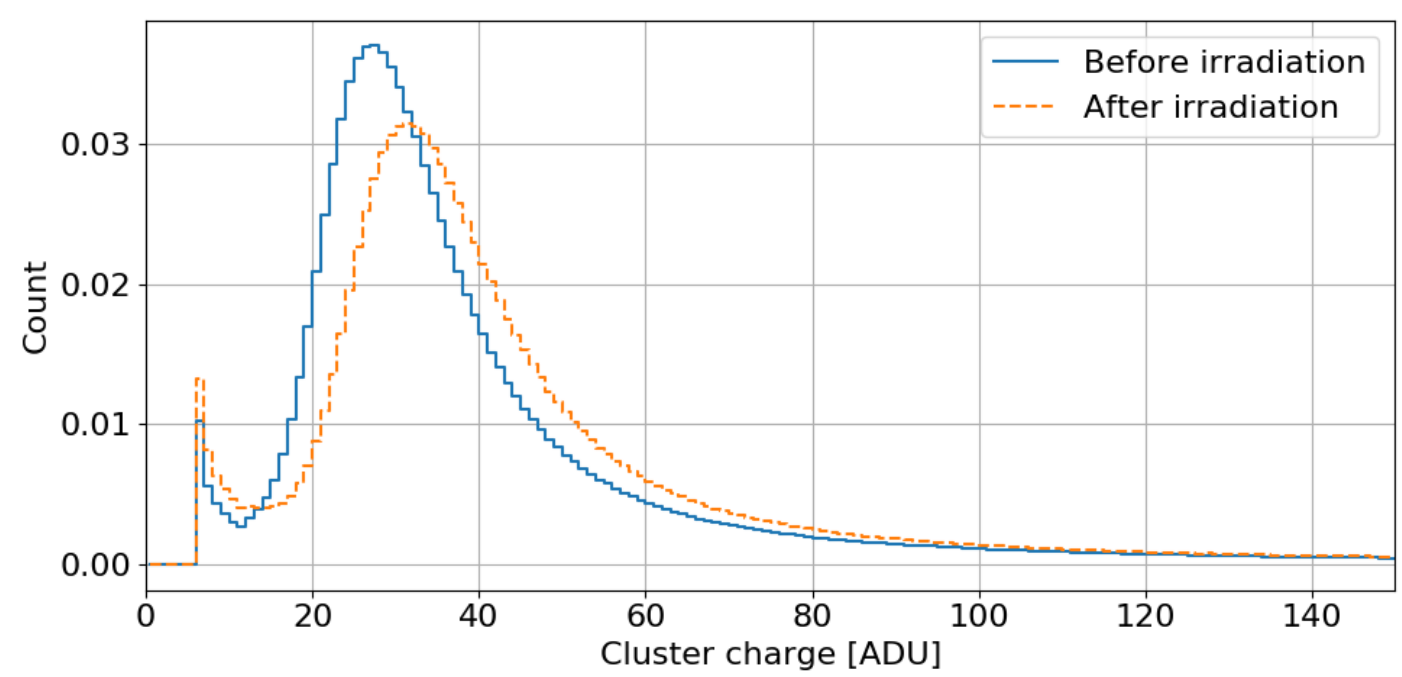

Figure 7.22.: Normalised cluster charge distributions before and after the irradiation campaign. For both measurements a ${ }^{90} \mathrm{Sr}$ source was used. Reprinted with permission from [87].

Landau distribution but are slightly shifted relative to each other because of a different working point setting. 


\subsubsection{Energy Calibration and DEPFET Working Point Evolution}

Using two different readioactive sources, it is possible to calculate the total gain of the sensor (DEPFET amplification + DCD amplification). To do this, the energy of the source has to be known and the sensor's response to it. For a $\gamma$ source the energy measured by the sensor has a gaussian shape with an MPV corresponding to the energy of the photons. For $\beta$ radiation with a continuous spectrum, this is more difficult. The energy deposition in Si detectors by charged particles can be described by a Landau distribution. The MPV of the Landau depends on the energy of the particle and the thickness of the material. To correctly model the response of the sensor, a Geant 4 simulation was created modelling a simplified version of the sensor $(75 \mu \mathrm{m}$ Si block $)$ and electrons coming from a particle gun with energies according to the ${ }^{90} \mathrm{Sr}$ spectrum. The ${ }^{90} \mathrm{Sr}$ spectrum was obtained via BetaShape [100] software, which is based on a theoretical model described in [101]. The expected shape of the spectrum measured by the sensor can be seen in fig. 7.23. From this simulation an MPV value of $19.4 \mathrm{keV}$ was extracted.

With this translation of the MPV values measured by the sensor in ADU to energies in $\mathrm{keV}$, the total gain of the sensor was calculated by performing a linear fit. This analysis was done for every source measurement made during the irradiation. Figure 7.24 shows an example of a fit to the data at the end of the irradiation. In order to see a possible gradient of the gain on the sensor, the analysis was done for different regions on the sensor as well. A map of the sensor before the irradiation can be seen in fig. 7.25. There is a visible gradient along the rows of the sensor, pixels in the switcher regions furthest away from the DCDs have the highest gain. This tendency was seen on other sensors as well during laboratory measurements.

As described in section 7.4, the target for the threshold shift compensation was the source current measured by the LMU power supply. This was meant to guarantee a constant working point of the DEPFETs. Figure 7.26 however shows that the total gain of the sensor was not constant during the irradiation. The average gain (over the whole sensor) was $\approx 1.4 \mathrm{ADU} / \mathrm{keV}$ at the beginning of the irradiation and $\approx 1.65 \mathrm{ADU} / \mathrm{keV}$ at the end. This behaviour indicates that the threshold shift was over-compensated by the readjustment of the gate voltage. It is important to remember that the gain of a single DEPFET cell is not affected by the irradiation, only the working point is shifted. Through the LMU power supply only the mean source current $\tilde{I}_{D}$ is known, which is the mean over the drain-source currents of all gates.

$$
\tilde{I}_{D} \propto \frac{1}{N} \sum_{i=1}^{N}\left(V_{G}-V_{\mathrm{thr}, i}\right)^{2}
$$

Keeping $\tilde{I}_{D}$ constant does not necessarily guarantee that the mean $g_{g} \propto V_{G}-V_{\text {thr }}$ is constant as well. This is especially the case when the threshold shifts $V_{\mathrm{thr}, i}$ of the gates vary, which is true due to the inhomogeneity of the irradiation.

Because only a global adjustment of the gate voltage was performed to keep $\tilde{I}_{D}$ con- 


\section{Irradiation Experiment}

stant, the working points of the DEPFETs across the sensor were shifted relative to each other. This can be seen clearly when looking at the evolution of the gain, shown in fig. 7.27. The figure shows the gains of the six switcher regions across the sensor. The regions at the border of the sensor, where less dose was received, show a larger gain increase than the central region.

At Belle II no such strong inhomogeneity in received dose is expected. Therefore, using the mean source current to keep the working point constant should be more reliable than in this measurement campaign.

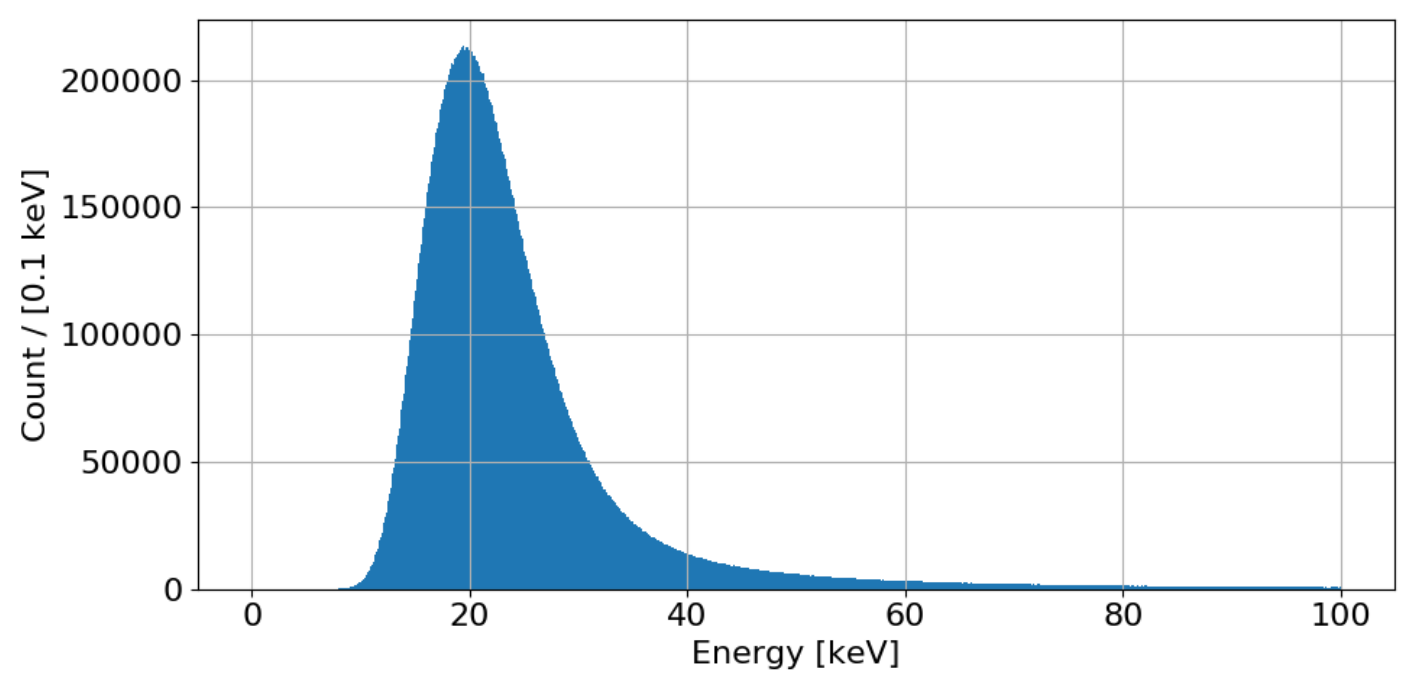

Figure 7.23.: Deposited energy of a ${ }^{90} \mathrm{Sr}$ beta source in $75 \mu \mathrm{m}$ Si, simulated with Geant4. 


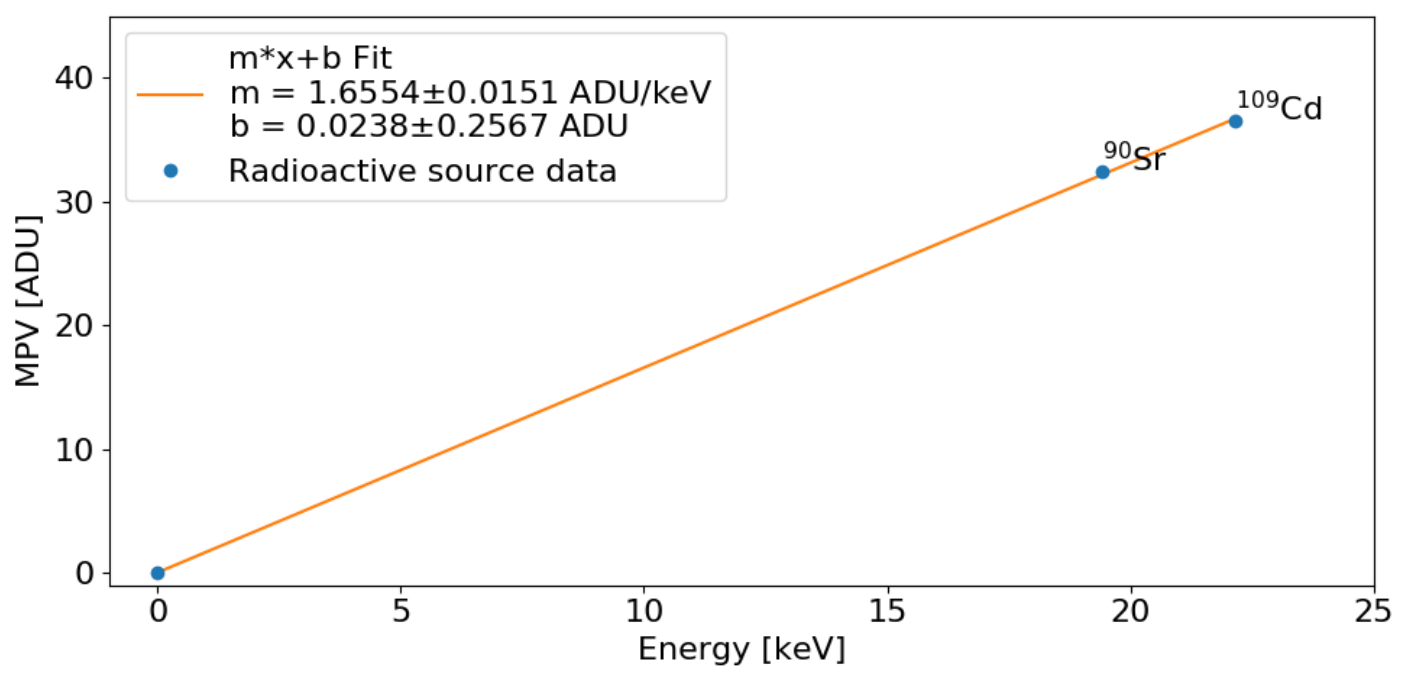

Figure 7.24.: Energy calibration at the end of the irradiation at $\approx 210 \mathrm{kGy}$.

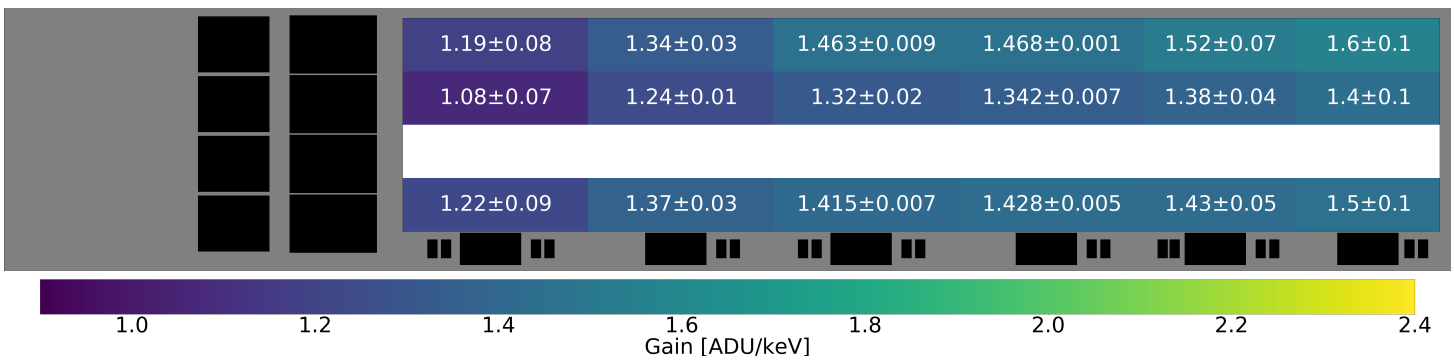

Figure 7.25.: System gain across the sensor at the start of the irradiation. The sensor is divided into ASIC/Switcher regions. The DCD-DHP pairs are located on the left side and the Switchers on the bottom side of the figure. No data of the third DCD-DHP pair was taken during the radioactive source measurements. 


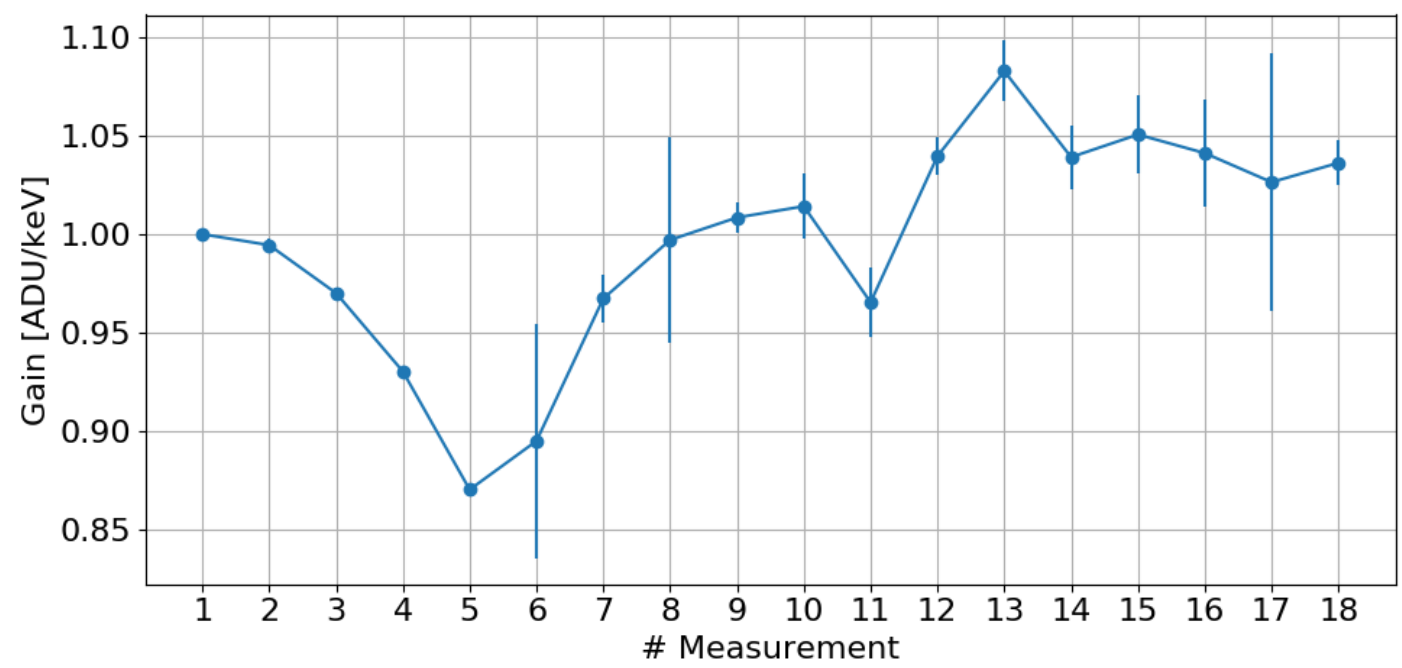

Figure 7.26.: Evolution of the energy calibration during the irradiation.

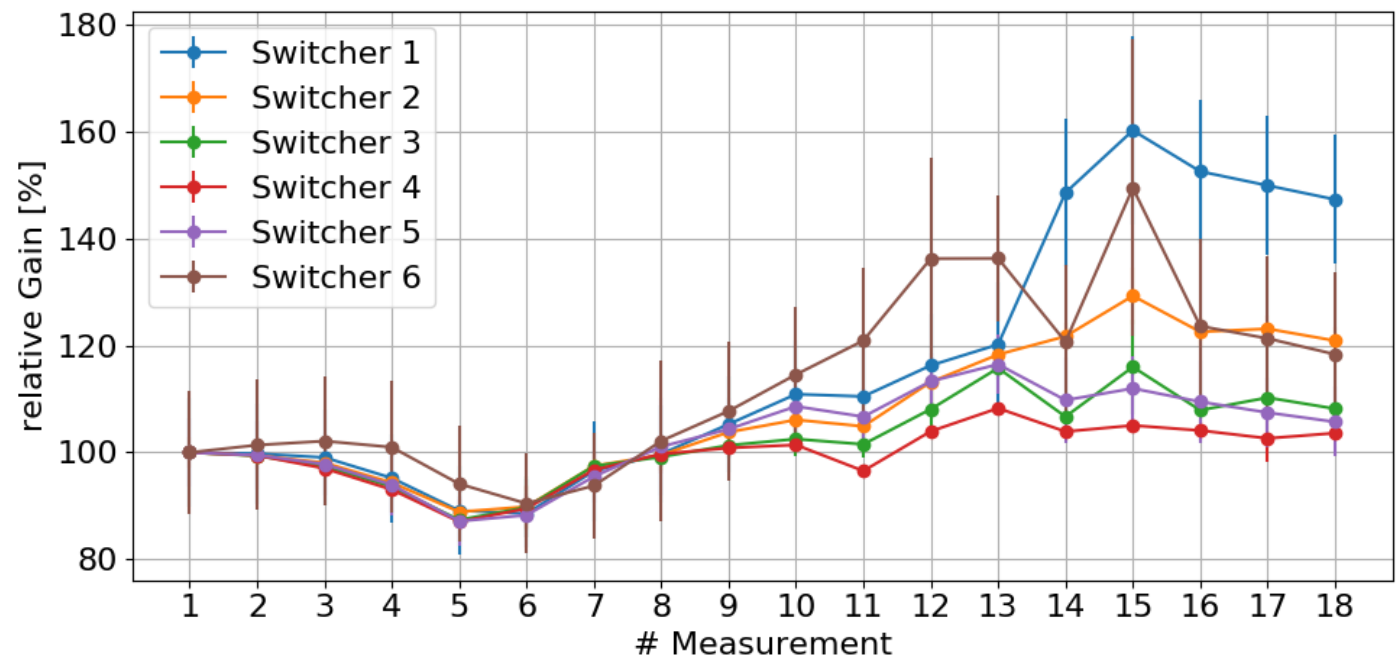

Figure 7.27.: Relative gain as a function of time. 


\subsubsection{Signal-to-Noise Ratio}

One of the most crucial observables by which the performance of the PXD is quantified is the signal-to-noise ratio (SNR), which can be estimated by using radioactive sources. The calculation of the SNR was done in the same manner as for the phase 2 PXD data, described in section 6.4.3. Figure 7.28 shows two examples of these SNR distributions at the beginning of the irradiation. As the noise across the sensor was rather homogeneous and stable over time, the form of the distribution is very similar to the cluster charge distribution.

As the SNR depends on the working point of the sensor, the same segmented analysis which was done for the sensor gain was repeated for the SNR as well. A map of the SNR across the sensor at the beginning of the irradiation can be seen in fig. 7.29.

The evolution of the SNR, which can be seen in Figure 7.30, is in turn very similar to the evolution of the system gain, discussed in section 7.8.1.

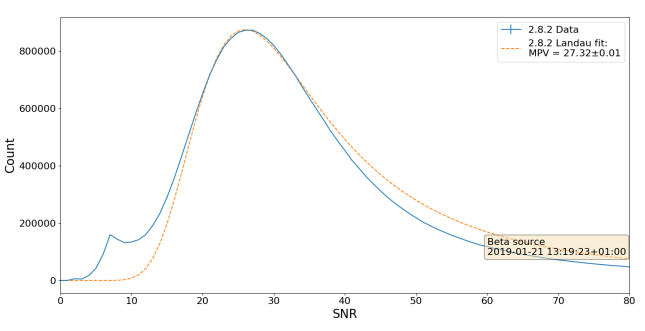

(a) ${ }^{90} \mathrm{Sr}$

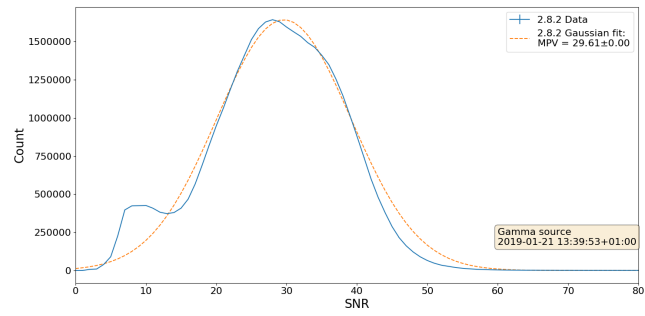

(b) ${ }^{109} \mathrm{Cd}$

Figure 7.28.: Signal to noise histograms for ${ }^{90} \mathrm{Sr}$ (left) and ${ }^{109} \mathrm{Cd}$ (right) with fitted Landau and Gaussian distribution, respectively.

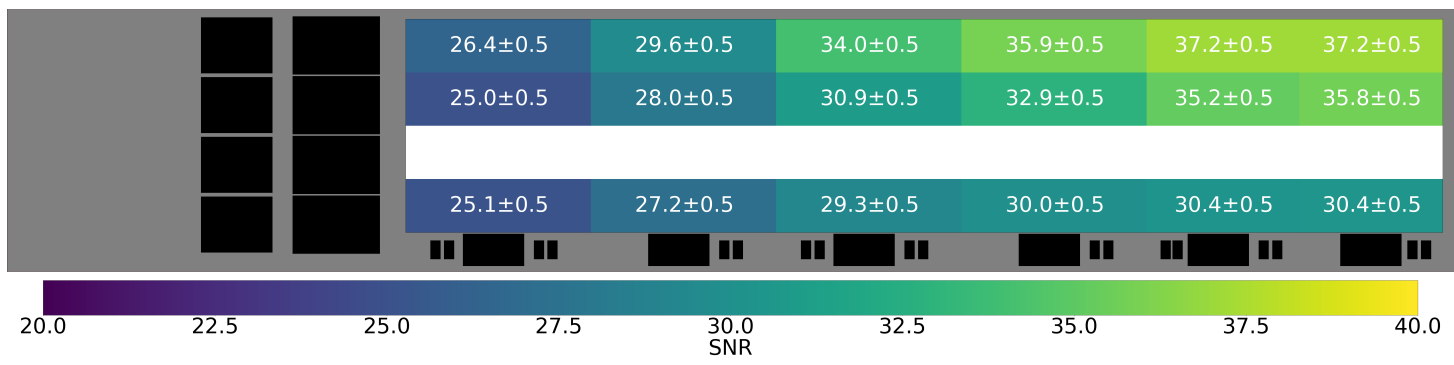

Figure 7.29.: SNR across the sensor at the beginning of the irradiation. The data was recorded with a ${ }^{90} \mathrm{Sr}$ source. 


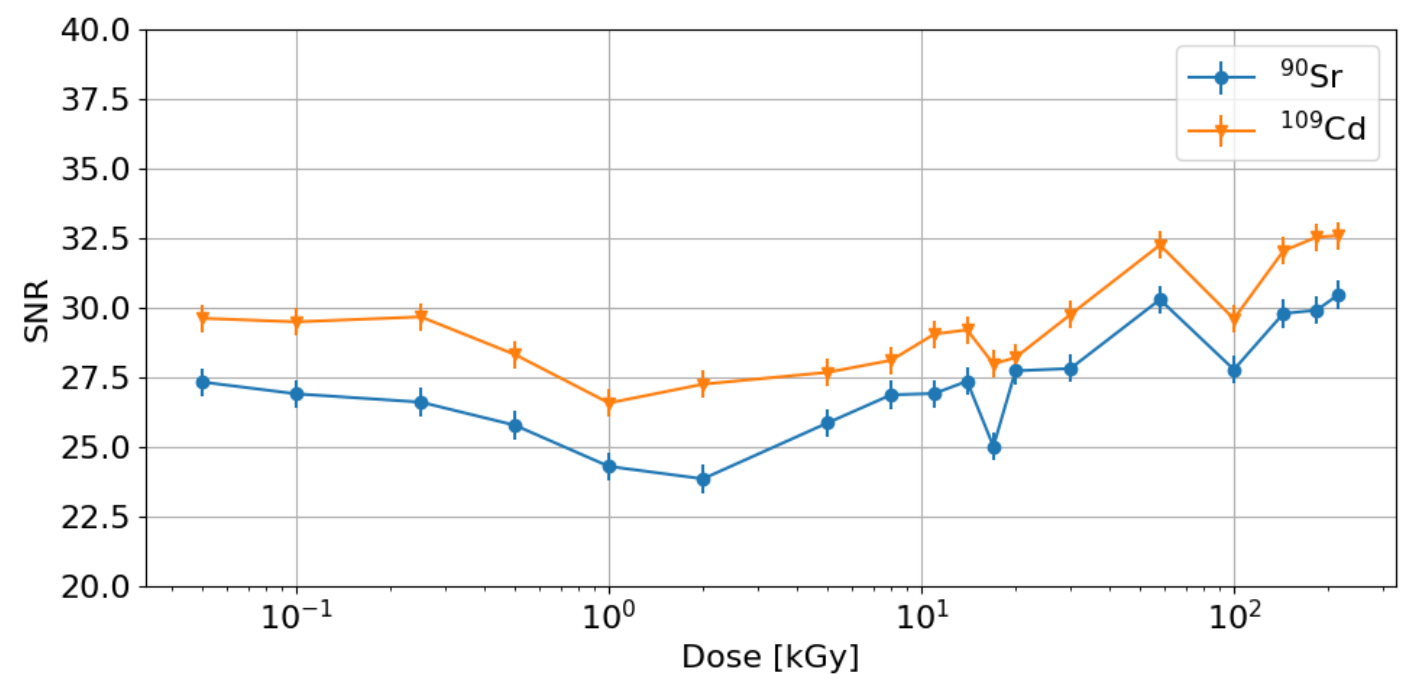

Figure 7.30.: Evolution of the signal-to-noise ratio during the irradiation. Reprinted with permission from [87].

\subsection{Efficiency Measurements with Beam Test Data}

Because only photons were used during the irradiation, the silicon bulk of the W05_OB1 half-ladder was not affected and no degradation of the hit detection efficiency was expected. In order to verify this, the half-ladder was tested during two beam tests at the DESY test beam facility (Hamburg, Germany) [102]. The first beam test was done before the irradiation and the second one afterwards to compare the performance directly.

The DESY test beam facility provides an electron beam with selectable energies between 1 and $6 \mathrm{GeV}$ and the necessary infrastructure to perform beam tests with arbitrary DUTs. During these beam tests multiple half-ladders were studied for their detection efficiency. Here only the results of the W05_OB1 half-ladder will be discussed. A complete description of these campaigns can be found in [103].

The DESY II accelerator is used to generate the beams for the test stands. Fiber targets are placed in the beam trajectories to create bremsstrahlung photons. These photons hit a secondary target and electron-positron pairs are created. Through focusing magnets the energy of the electrons/positrons can be restricted to match the user's needs. An overview of the test beam facility can be seen in fig. 7.31. For the efficiency measurements a EUDET-type beam telescope [104] consisting of six Mimosa26 monolithic active pixel sensor planes was used. The DUT is placed in the middle of the telescope with three Mimosa26 planes upstream and three planes downstream. A sketch of a typical telescope setup can be seen in fig. 7.32. For the data acquisition during the beam test a laboratory type readout system without a DHC was used for the half-ladder. The Miomosa26 planes have a long integration time of $115.2 \mu$ s compared 


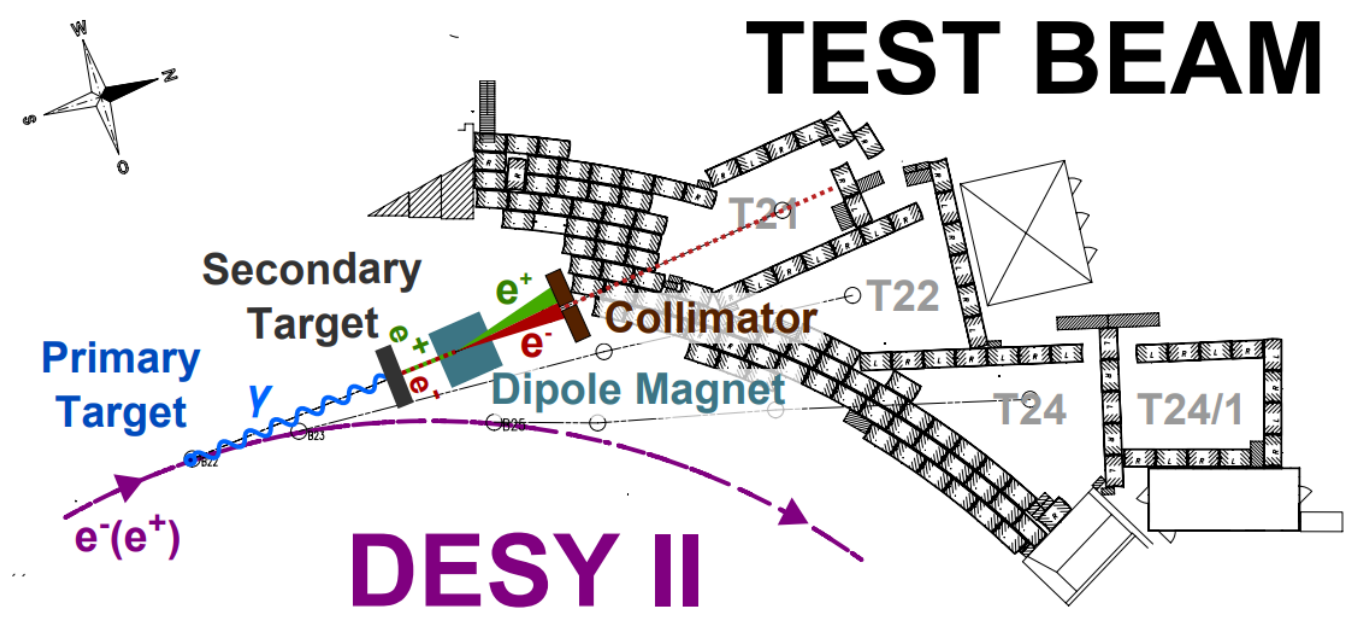

Figure 7.31.: Overview of the Desy test beam facility. From [102].

to the $20 \mu \mathrm{s}$ of a PXD half-ladder. This means that whenever the readout is triggered there are several tracks in one event and it is non-trivial to identify the track that caused the trigger. Therefore, the triggering system consisted of an FE-I4 frond-end read-out chip [105] with an integration time of $25 \mathrm{~ns}$ and scintillator planes at both ends of the telescope. A trigger was generated whenever at least one of the FE-I4 pixels recorded a hit in coincidence with the scintillators. The FE-I4 sensor was also used as an additional tracking plane. Reconstruction of the particle tracks was done with the Testbeam Software Framework (TBWS) [106]. The framework uses a Kalman Filter [107] approach to reconstruct the tracks between the individual sensor planes. The software supports multiple position finding algorithms like center-of-gravity, which is the algorithm used for the analysis presented here. A detailed description of TBSW and its functionalities can be found in $[108,109]$.

Since the beam spot size at the DESY stands is smaller than the size of the sensor, the sensor was only partly illuminated. To achieve a full illumination the sensor was moved during the test campaigns. Each position defined an experiment, which consists of several runs. Each of these runs had typically 200k - 500k events. A combination of the experiments was used to calculate the detection efficiency across the whole sensor. For the first beam test the energy of the beam was set to $3 \mathrm{GeV}$ and for the second one to $5 \mathrm{GeV}$.

The detection efficiency $\epsilon$ is defined as the number of tracks with an associated hit on the half-ladder $k$ divided by the total number of tracks $n$ registered by the EUDET telescope. To calculate the hit efficiency, an occupancy based pixel masking was performed and macro pixels with a size of 15 columns x 16 rows were defined to increase the statistic. Data from the third DHP-DCD pair was not considered for the analysis, anal- 


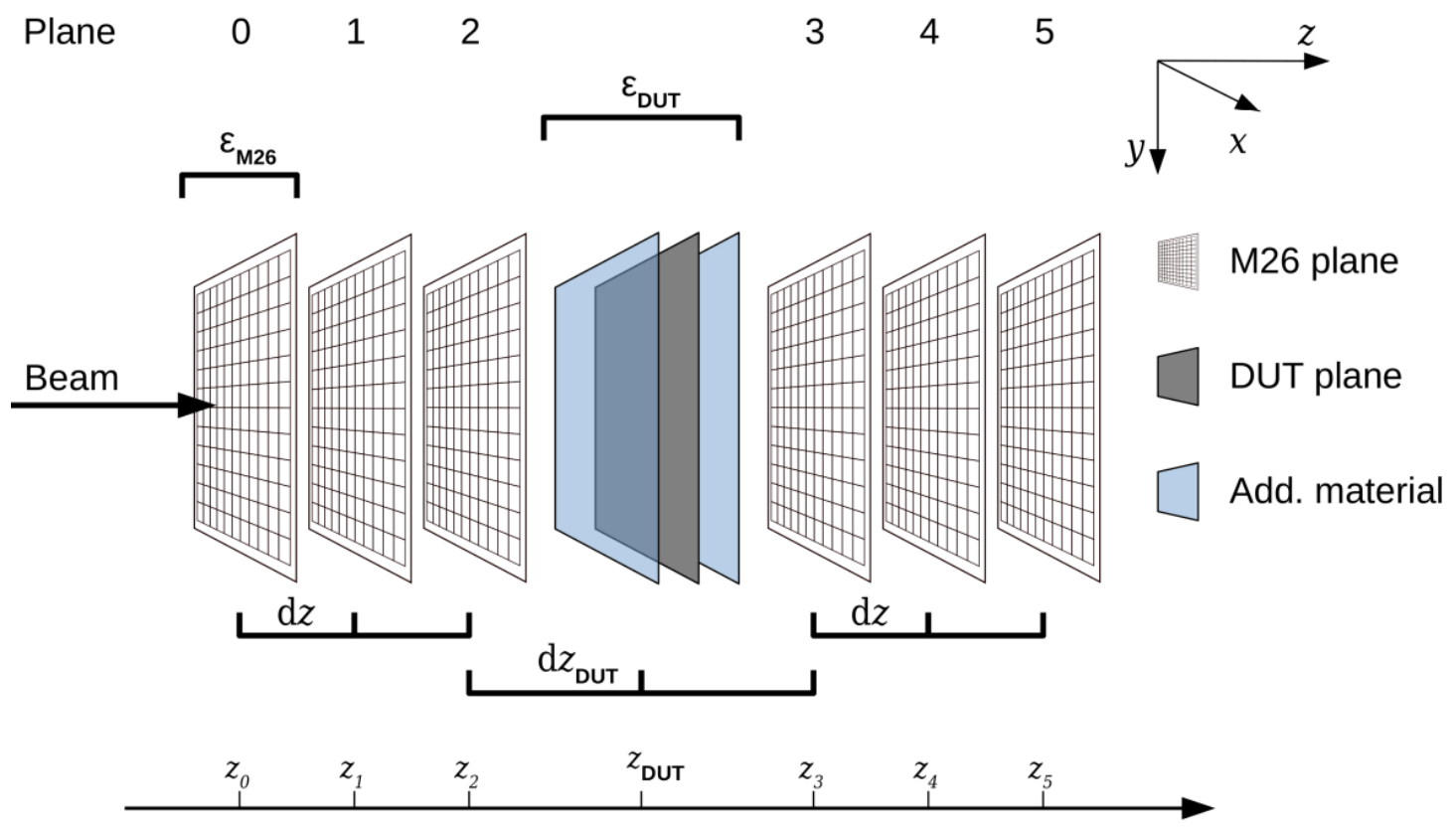

Figure 7.32.: Sketch of a EUDET-type beam telescope. From [104].

ogous to the I-V curve measurements. The preliminary efficiencies (mean over the entire sensor without masked pixels) before and after the irradiation as well as the noise (mean pedestal noise), the SNR and the masked pixel fraction are summarised in table 7.5. As

\begin{tabular}{l|c|c|c|c|c}
\hline & Efficiency [\%] & Noise [ADU] & ENC [e ${ }^{-}$] & SNR & Masked Pixels [\%] \\
\hline Before & $99.69 \pm 0.04$ & 0.62 & 125 & 42.9 & 0.53 \\
After & $99.54 \pm 0.01$ & 0.63 & 110 & 51.4 & 8.3 \\
\hline
\end{tabular}

Table 7.5.: Overview of hit efficiency, noise, equivalent noise charge (ENC), SNR and masked pixel fraction before and after the irradiation. Because of a different working point after the irradiation, the SNR is increased and the ENC is decreased. Reprinted with permission from [87].

previously stated, no influence on the charge collection and on the detection efficiency is expected as there is no bulk damage. This is also what is seen when comparing the efficiencies from the first and second beam test. The noise is also comparable between the two measurements. Due to a different working point (see section 7.8.1) the SNR and the ENC differ slightly. The biggest difference is the number of masked pixels. Because of an operator's mistake during the irradiation campaign some pixel rows were electrically damaged, which leads to an increased number of unusable and therefore masked pixels. The inhomogeneity of the irradiation made it also difficult to get all pixels into the dynamic range of the DCD. Both of these effects are directly linked to this particular 
irradiation campaign. For the operation of Belle II they are irrelevant. Figure 7.33 shows a one dimensional projection of the efficiencies before and after the irradiation. As the third DHP-DCD pair was not used, the illumination of the half-ladder was restricted to the first two DHP-DCD pairs (columns 1 - 125). The figure shows that the efficiency of the pixels was slightly lower after the irradiation but still around $99.6 \%$.

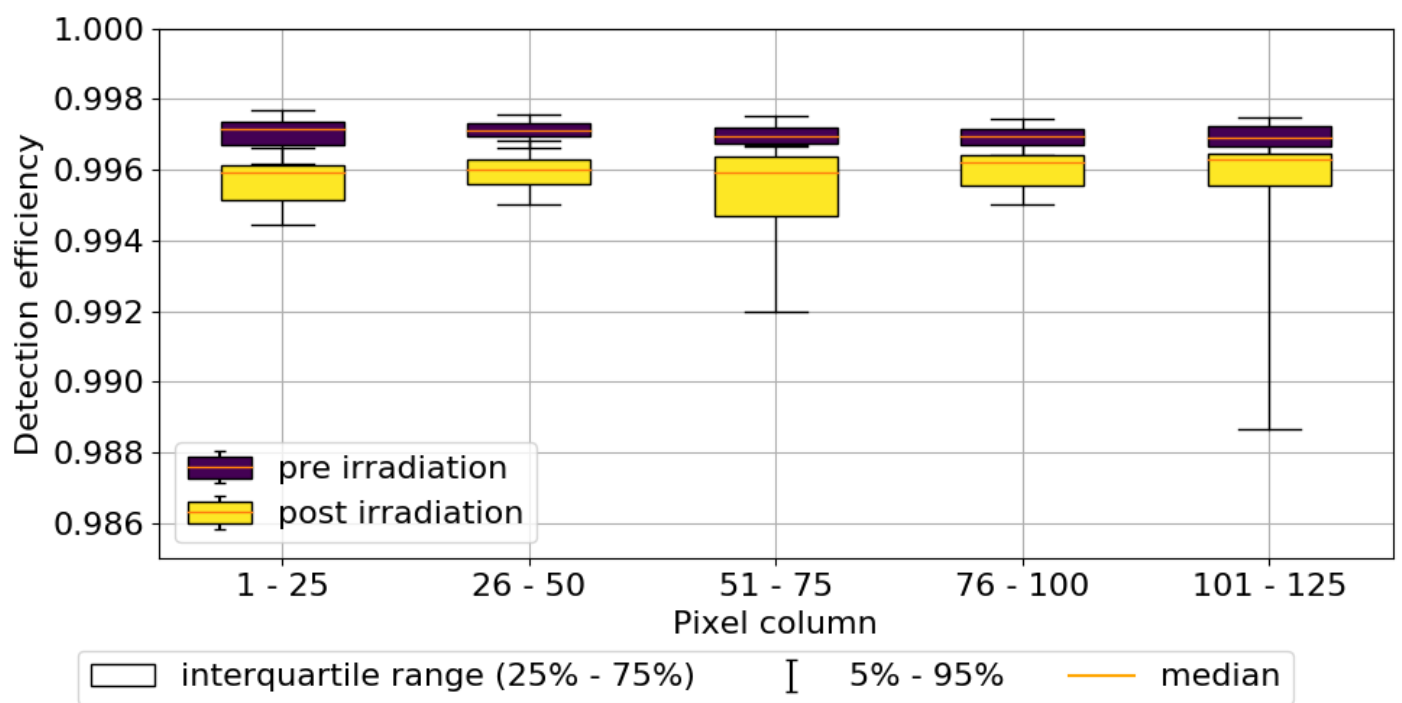

Figure 7.33.: Projection of the detection efficiencies before (black) and after (green) the irradiation along the u-axis (pixel columns). Only one half of the half-ladder was illuminated during the measurements (columns 1 - 125). Reprinted with permission from [87]. 



\section{CHAPTER 8}

\section{Conclusion}

In this thesis the transition from a laboratory DEPFET PXD system to a final-like configuration at Belle II was presented.

This transition required not only an adjustment of the hardware but also a major rework of the software that was used in the laboratories to calibrate single DEPFET devices. This rework included adjustments of existing software, but also the creation of new EPICS based IOCs to be able to control, monitor and calibrate the four PXD sensors installed during the phase 2 operation. An important aspect of this software rework was the creation of tools that are easily expandable to a full setup with 40 PXD half-ladders. Furthermore, the tools dedicated to the calibration of sensors were constructed in a way that allows an easy use for non-experts which will operate the phase 3 system in the coming years. The Calibration IOC fulfils all these requirements and has proven to be a helpful tool during the day-to-day operation during phase 2. Since early 2019, the phase 3 operation at Belle II has started and the Calibration IOC is still being used there.

This thesis also describes the development and usage of an automatic logging system for laboratory measurements and calibrations as well as the regular data taking operation at Belle II. The software libraries written for the python based framework allowed to standardise the documentation of crucial calibration measurements, which was especially relevant for the mass testing of phase 3 PXD sensors that was done in parallel to the phase 2 operation at KEK.

The elog messenger reliably recorded the phase 2 runs and reduced the danger of wrong documentation due to human error. The PXD specific fork of the messenger controlled the local PXD DAQ which allowed to automatise the local data taking and synchronise it with the global Belle II DAQ. It was this data that was analysed in this thesis. Like the Calibration IOC, it is used during the ongoing phase 3 operation. The built-in redundancy features have already been proven to be useful on various occasions. The same type of messenger was also used during the test beam measurements described 


\section{Conclusion}

in section 7.9. The logbook entries from these measurement campaigns have proven to be very helpful in the analysis as well.

Apart from combined test beam campaigns where SVD ladders and PXD ladders were tested together, phase 2 marked the first time multiple full PXD half-ladders were used for data taking over a longer period of time. While one of the modules had a defective high-speed data link that disabled the readout of one quarter of its DEPFET matrix, the sensors performed exceptionally well. The threshold voltage shift of the DEPFETs was larger than expected because the backgrounds were underestimated in the initial simulations. Nevertheless, the gate voltages of the sensors could be adjusted to compensate the effect of the radiation and keep the the working point constant resulting in a stable SNR over 50 for all four PXD sensors.

The observed threshold voltage shift and its incompatibility with the dose rates measured by the Belle II diamond beam monitor system led to the idea to use the PXD data to get a dose estimate for the phase 2 duration. As the analysis in this thesis showed, such an estimation is possible using few assumptions such as a negligible difference between triggered and un-triggered data. According to the calculated estimation the inner PXD ladders had received a dose of $2 \mathrm{kGy}$ and the outer ladders a dose of $1 \mathrm{kGy}$. This estimation is much more compatible with previous irradiation measurements as was shown in section 6.5.

As a result of the experience with the PXD sensors during phase 2 and their observed threshold voltage shift, a final-type sensor was irradiated with an X-ray source to a dose of $266 \mathrm{kGy}$. Based on the initial simulations this dose should correspond to 10 years of Belle II operation. This was the very first time that a complete PXD half-ladder with final-type ASICs and a matrix that comes from the same production batch as the ones used for Belle II was irradiated as a whole. As the analyses presented in this thesis show the sensor was fully functional after this irradiation. The observed threshold voltage shifts of up to $10 \mathrm{~V}$ could be compensated and the SNR of the sensor was stable as well. The maximum tolerable voltage shift is limited by the electronics of the half-ladder, particular by the switchers. A shift of at least $20 \mathrm{~V}$ can be compensated which leaves sufficient head-room for even higher radiation doses. The analysis did, however, show that an inhomogeneous irradiation can make it difficult to keep the working point of the DEPFETs stable over the entire sensor. At Belle II such a strong inhomogeneity is not expected and in addition the issue can be partly resolved by adjusting the three gate voltages per sensor individually which was not done during the irradiation campaign.

While the ASICs received a significantly lower dose $(\leq 20 \mathrm{kGy})$ than the DEPFETs due to the soft spectrum and their shielding, they were still affected as the analyses showed. However, the effects were minor and had no influence on their performance.

Two beam test campaigns done with the half-ladder that was used for the irradiation campaign verified that the hit detection efficiency of the sensor showed only a very slight overall degradation and went from $99.69 \pm 0.04 \%$ to $99.54 \pm 0.01 \%$. The pedestal noise was nearly identical as well. As the X-ray irradiation did not cause any bulk damage this result was expected, but the outcome of the beam test campaign was nevertheless a good verification. 
It is the bulk damage that remains an open question after the measurement and analyses documented here. Even though the phase 3 operation of Belle II has already started, a dedicated measurement campaign with a neutron source to study bulk damage in detail is currently under discussion. While previous studies with prototype DEPFET structures have shown that leakage current and type inversion are not a concern for the operation at Belle II, a dedicated study with a final-type half ladder might provide interesting insights. 

[1] Griffiths, David J., Introduction to elementary particles. Wiley-VCH, 2nd ed., 2008. (Cited on page 3.)

[2] M. Tanabashi et al., Review of Particle Physics, Physical Review D 98 (2018). (Cited on pages 4, 7, 9, and 17.)

[3] O. S. Brüning, P. Collier, P. Lebrun, S. Myers, R. Ostojic, J. Poole, and P. Proudlock, LHC Design Report. CERN Yellow Reports: Monographs. CERN, Geneva, 2004. (Cited on pages 6 and 79.)

[4] ATLAS Collaboration, G. Aad et al., Observation of a new particle in the search for the Standard Model Higgs boson with the ATLAS detector at the LHC, Phys. Lett. B716 (2012) 1-29. (Cited on page 6.)

[5] CMS Collaboration, S. Chatrchyan et al., Observation of a New Boson at a Mass of $125 \mathrm{GeV}$ with the CMS Experiment at the LHC, Phys. Lett. B716 (2012) 30-61. (Cited on page 6.)

[6] P. W. Higgs, Broken Symmetries and the Masses of Gauge Bosons, Phys. Rev. Lett. 13 (1964) 508-509. (Cited on page 6.)

[7] F. Englert and R. Brout, Broken Symmetry and the Mass of Gauge Vector Mesons, Phys. Rev. Lett. 13 (1964) 321-323. (Cited on page 6.)

[8] G. S. Guralnik, C. R. Hagen, and T. W. B. Kibble, Global Conservation Laws and Massless Particles, Phys. Rev. Lett. 13 (1964) 585-587. (Cited on page 6.)

[9] N. Cabibbo, Unitary Symmetry and Leptonic Decays, Phys. Rev. Lett. 10 (1963) 531-533. (Cited on page 7.)

[10] M. Kobayashi and T. Maskawa, CP Violation in the Renormalizable Theory of Weak Interaction, Prog. Theor. Phys. 49 (1973) 652-657. (Cited on page 7.) 


\section{BIBLIOGRAPHY}

[11] L.-L. Chau and W.-Y. Keung, Comments on the Parametrization of the Kobayashi-Maskawa Matrix, Physical Review Letters 53 (1984) 1802-1805. (Cited on page 8.)

[12] L. Wolfenstein, Parametrization of the Kobayashi-Maskawa Matrix, Physical Review Letters 51 (1983) 1945-1947. (Cited on page 8.)

[13] E. Noether, Invariante Variationsprobleme, Nachrichten von der Gesellschaft der Wissenschaften zu Göttingen, Mathematisch-Physikalische Klasse 1918 (1918) 235-257. (Cited on page 10.)

[14] C. S. Wu, E. Ambler, R. W. Hayward, D. D. Hoppes, and R. P. Hudson, Experimental Test of Parity Conservation in Beta Decay, Phys. Rev. 105 (1957) 1413-1414. (Cited on page 10.)

[15] J. H. Christenson, J. W. Cronin, V. L. Fitch, and R. Turlay, Evidence for the $2 \pi$ Decay of the $K_{2}^{0}$ Meson, Phys. Rev. Lett. 13 (1964) 138-140. (Cited on page 10.)

[16] BaBar Collaboration, B. Aubert et al., Measurement of CP violating asymmetries in $B^{0}$ decays to CP eigenstates, Phys. Rev. Lett. 86 (2001) 2515-2522. (Cited on page 10.)

[17] Belle Collaboration, K. Abe et al., Observation of large CP violation in the neutral B meson system, Phys. Rev. Lett. 87 (2001) 091802. (Cited on page 10.)

[18] Y. Chao et al., Evidence for Direct CP Violation in $B^{0} \rightarrow K^{+} \pi^{-}$Decays, Phys. Rev. Lett. 93 (2004). (Cited on page 11.)

[19] BaBar Collaboration, D. Boutigny et al., BaBar technical design report, in BaBar Technical Design Report EPAC Meeting Stanford, California, March 17-18, 1995. 1995. (Cited on page 13.)

[20] A. Abashian et al., The Belle detector, Nucl. Instrum. Methods Phys. Res., Sect. A 479 (2002) 117-232. (Cited on page 13.)

[21] LHCb Collaboration, A. A. Alves et al., The LHCb Detector at the LHC, J. Instrum. 3 (2008) S08005-S08005. (Cited on page 14.)

[22] HFLAV Collaboration, Y. S. Amhis et al., Averages of b-hadron, c-hadron, and $\tau$-lepton properties as of 2018, arXiv:1909.12524 [hep-ex]. (Cited on page 15.)

[23] E. Kou et al., The Belle II Physics Book, Progress of Theoretical and Experimental Physics 2019 (2019). (Cited on page 15.)

[24] Y. Ohnishi et al., Accelerator design at SuperKEKB, Prog. Theor. Exp. Phys. 2013 (2013). (Cited on pages 17, 19, 21, and 22.)

[25] T. KEK, KEKB B factory design report,. (Cited on page 17.) 
[26] SuperKEKB Collaboration, K. Akai, K. Furukawa, and H. Koiso, SuperKEKB Collider, Nucl. Instrum. Meth. A907 (2018) 188-199. (Cited on pages 17, 18, and 19.)

[27] S. Hashimoto, M. Hazumi, J. Haba, J. W. Flanagan, Y. Ohnishi, K. Abe, K. Abe, T. Abe, I. Adachi, T. Agoh, et al., Letter of intent for KEK Super B Factory,. (Cited on page 19.)

[28] SuperB Collaboration, M. Bona et al., SuperB: A High-Luminosity Asymmetric e+ e-Super Flavor Factory. Conceptual Design Report,. (Cited on page 19.)

[29] C. Bernardini, G. Corazza, G. Di Giugno, G. Ghigo, J. Haissinski, P. Marin, R. Querzoli, and B. Touschek, Lifetime and beam size in a storage ring, Phys. Rev. Lett. 10 (1963) 407. (Cited on page 20.)

[30] A. Piwinski, The Touschek effect in strong focusing storage rings, physics/9903034, DESY-98-179. (Cited on page 20.)

[31] S. Di Carlo, P. Bambade, D. Jehanno, V. Kubytskyi, C. Pang, Y. Peinaud, and C. Rimbault, Early Phase 2 Results of LumiBelle2 for the SuperKEKB Electron Ring, J. Phys. Conf. Ser. 1067 (2018) 072025. (Cited on page 21.)

[32] P. Lewis et al., First measurements of beam backgrounds at SuperKEKB, Nucl. Instrum. Methods Phys. Res. A 914 (2019) 69 - 144. (Cited on pages 21 and 82.)

[33] Belle-II Collaboration, T. Abe et al., Belle II Technical Design Report, arXiv:1011.0352 [physics.ins-det]. (Cited on pages 24, 26, and 27.)

[34] Belle II Detector, https://www.belle2.org/project/super_kekb_and_belle_ii/. (retrieved on 10.02.2020). (Cited on page 24.)

[35] Belle-II SVD Collaboration, K. R. Nakamura et al., The Belle II SVD detector, PoS Vertex2016 (2017) 012. (Cited on page 25.)

[36] H. Ye, C. Niebuhr, R. Stever, K. Gadow, and C. Camien, Thermal mock-up studies of the Belle II vertex detector, Nucl. Instrum. Meth. A 896 (2018) 82-89. (Cited on pages 25 and 110.)

[37] K. C. S. Adel S. Sedra, Microelectronic Circuits. Oxford University Press, 5 ed., 2004. (Cited on page 32.)

[38] J. Kemmer and G. Lutz, New detector concepts, Nucl. Instrum. Methods Phys. Res. A 253 (1987) 365 - 377. (Cited on page 36.)

[39] L. Andricek et al., Advanced testing of the DEPFET minimatrix particle detector, J. Instrum. 7 (2012) C01101-C01101. (Cited on page 36.) 


\section{BIBLIOGRAPHY}

[40] A. Moll, Comprehensive study of the background for the Pixel Vertex Detector at Belle II. PhD thesis, Ludwig-Maximilians-Universität München, 2015. (Cited on page 38.)

[41] A. Ritter, Radiation Hardness Studies for DEPFETs in Belle II . PhD thesis, Technische Universität München, 2014. (Cited on page 39.)

[42] G. A. Ausman Jr and F. B. McLean, Electron- hole pair creation energy in SiO2, Appl. Phys. Lett. 26 (1975) 173-175. (Cited on page 39.)

[43] T. R. Oldham, Recombination along the tracks of heavy charged particles in SiO2 films, J. Appl. Phys. 57 (1985) 2695-2702. (Cited on page 39.)

[44] T. Oldham and J. McGarrity, Comparison of 60Co Response and $10 \mathrm{KeV} \mathrm{X-ray}$ response in MOS capacitors, IEEE Trans. Nucl. Sci. 30 (1983) 4377-4381. (Cited on page 39.)

[45] R. Hughes, Charge-Carrier Transport Phenomena in Amorphous Si O 2: Direct Measurement of the Drift Mobility and Lifetime, Phys. Rev. Lett. 30 (1973) 1333. (Cited on page 39.)

[46] H. E. Boesch and F. B. McLean, Hole transport and trapping in field oxides, IEEE Trans. Nucl. Sci. 32 (1985) 3940-3945. (Cited on page 39.)

[47] G. Pfister and H. Scher, Dispersive (non-Gaussian) transient transport in disordered solids, Adv. Phys. 27 (1978) 747-798. (Cited on page 40.)

[48] R. Velazco, P. Fouillat, and R. Reis, Radiation effects on embedded systems. Springer Science \& Business Media, 2007. (Cited on page 40.)

[49] P. M. Lenahan and P. Dressendorfer, Hole traps and trivalent silicon centers in metal/oxide/silicon devices, J. Appl. Phys. 55 (1984) 3495-3499. (Cited on page 40.)

[50] F. McLean, A framework for understanding radiation-induced interface states in SiO2 MOS structures, IEEE Trans. Nucl. Sci. 27 (1980) 1651-1657. (Cited on page 40.)

[51] N. Saks and D. Brown, Interface trap formation via the two-stage H/sup+/process, IEEE Trans. Nucl. Sci. 36 (1989) 1848-1857. (Cited on page 40.)

[52] J. Prinzie, M. Steyaert, and P. Leroux, Radiation Hardened CMOS Integrated Circuits for Time-Based Signal Processing. Springer, 2018. (Cited on page 41.)

[53] J. Schwank, P. Winokur, P. McWhorter, F. Sexton, P. Dressendorfer, and D. Turpin, Physical Mechanisms Contributing to Device" Rebound", IEEE Trans. Nucl. Sci. 31 (1984) 1434-1438. (Cited on page 41.) 
[54] A. Lelis, H. Boesch, T. Oldham, and F. McLean, Reversibility of trapped hole annealing, IEEE Trans. Nucl. Sci. 35 (1988) 1186-1191. (Cited on page 41.)

[55] A. Lelis, T. Oldham, H. Boesch, and F. McLean, The nature of the trapped hole annealing process, IEEE Trans. Nucl. Sci. 36 (1989) 1808-1815. (Cited on page 41.)

[56] A. J. Lelis and T. R. Oldham, Time dependence of switching oxide traps, IEEE Trans. Nucl. Sci. 41 (1994) 1835-1843. (Cited on page 41.)

[57] I. Peric, P. Fischer, J. Knopf, and T. Nguyen, DCDB and SWITCHERB, the readout ASICs for Belle II DEPFET pixel detector, IEEE Nucl. Sci. Symp. Conf. Rec (2011) 1536-1539. (Cited on pages 42 and 46.)

[58] H. Krüger, Front-end electronics for DEPFET pixel detectors at SuperBelle (BELLE II), Nucl. Instrum. Methods Phys. Res. A 617 (2010) 337 - 341. (Cited on page 42.)

[59] R. H. Richter et al., Design and technology of DEPFET pixel sensors for linear collider applications, Nucl. Instrum. Methods Phys. Res. A 511 (2003) 250-256. (Cited on page 43.)

[60] F. Müller, Characterization and optimization of the prototype DEPFET modules for the Belle II Pixel Vertex Detector. PhD thesis,

Ludwig-Maximilians-Universität München, 2017. (Cited on pages 44 and 45.)

[61] P. Wieduwilt, Optimization of the front-end read-out electronics for the Belle II DEPFET Sensor, Master's thesis, Universität Göttingen, 2016. (Cited on page 48.)

[62] D. Levit, I. Konorov, D. Greenwald, and S. Paul, FPGA based data read-out system of the Belle II pixel detector, IEEE Trans. Nucl. Sci. 62 (2015) 1033-1039. (Cited on pages 52 and 53.)

[63] M. Nakao, C. Lim, M. Friedl, and T. Uchida, Minimizing dead time of the Belle II data acquisition system with pipelined trigger flow control, in 2012 18th IEEE-NPSS Real Time Conference. IEEE, Jun, 2012. (Cited on page 53.)

[64] T. Gesler, W. J. Kuhn, J. S. Lange, Z. Liu, D. Munchow, D. R. Perez, and J. Zhao, The ONSEN Data Reduction System for the Belle II Pixel Detector, IEEE Trans. Nucl. Sci. 62 (2015) 1149-1154. (Cited on pages 53 and 54.)

[65] R. Itoh, T. Higuchi, M. Nakao, S. Y. Suzuki, and S. Lee, Data Flow and High Level Trigger of Belle II DAQ System, IEEE Transactions on Nuclear Science 60 (2013) 3720-3724. (Cited on page 53.)

[66] F. J. Lütticke, Development of Pixel Modules for the Belle II Detector. PhD thesis, Rheinische Friedrich-Wilhelms-Universität Bonn, 2017. (Cited on pages 55 and 128.) 


\section{BIBLIOGRAPHY}

[67] S. Rummel, The power supply system for the DEPFET pixel detector at BELLE II, Nucl. Instrum. Methods Phys. Res. A 699 (2013) 51 - 55. (Cited on page 55.)

[68] Experimental Physics and Industrial Control System (EPICS), https://epics-controls.org/. (Cited on page 59.)

[69] Control System Studio, http://controlsystemstudio.org/. (Cited on page 61.)

[70] M. Shankar, M. Davidsaver, M. Konrad, and L. Li, The EPICS Archiver Appliance, in Proceedings, 15th International Conference on Accelerator and Large Experimental Physics Control Systems (ICALEPCS 2015): Melbourne, Australia, October 17-23, 2015. 2015. (Cited on page 61.)

[71] R. Frazier, G. Iles, D. Newbold, and A. Rose, Software and firmware for controlling CMS trigger and readout hardware via gigabit Ethernet, Physics Procedia 37 (2012) 1892-1899. (Cited on page 62.)

[72] F. A. Ben Franksen, State Notation Language and Sequencer, https://www-csr.bessy.de/control/SoftDist/sequencer/. (Cited on page 63.)

[73] M. Newville, PyEpics: Epics Channel Access for Python, https://cars9.uchicago.edu/software/python/pyepics3/index.html. (Cited on page 68.)

[74] L. Germic, Data Handling Processor and Signal Transmission in the Belle II DEPFET Pixel Detector. PhD thesis, Rheinische Friedrich-Wilhelms-Universität Bonn, 2018. (Cited on page 68.)

[75] X. Wang, PCASPy, https://pypi.org/project/pcaspy/. (Cited on page 69.)

[76] S. Ritt, ELOG, https://elog.psi.ch/elog. (Cited on page 72.)

[77] T. Konno, R. Itoh, M. Nakao, S. Suzuki, and S. Yamada, The Slow Control and Data Quality Monitoring System for the Belle II Experiment, IEEE Trans. Nucl. Sci. 62 (2015) 1-6. (Cited on page 76.)

[78] ATLAS IBL Collaboration, J. Albert et al., Prototype ATLAS IBL Modules using the FE-I4A Front-End Readout Chip, J. Instrum. 7 (2012) P11010. (Cited on page 79.)

[79] M. Garcia-Sciveres et al., The FE-I4 pixel readout integrated circuit, Nucl. Instrum. Methods Phys. Res. A 636 (2011) S155 - S159. (Cited on page 79.)

[80] P. Ahlburg, Development of a FE-I4-based module for radiation monitoring with BEAST II during the commissioning phase of the Belle II detector., Master's thesis, Rheinische Friedrich-Wilhelms-Universität Bonn, 2018. (Cited on page 79.) 
[81] D. Heuchel, CLAWS in BEAST Phase II: Investigation of the Beam Background in the Commissioning of SuperKEKB, Master's thesis, TU München, 2018. (Cited on page 80.)

[82] A. Nomerotski et al., PLUME collaboration: Ultra-light ladders for linear collider vertex detector, Nucl. Instrum. Methods Phys. Res., Sect. A 650 (2011) 208 212, International Workshop on Semiconductor Pixel Detectors for Particles and Imaging 2010. (Cited on page 80.)

[83] J. Baudot et al., BEAST results on SuperKEKB beam background: focus on the PLUME pixelated system, Dec., 2018. https://indico.cern.ch/event/669866/contributions/3242084. (Cited on page 80. .)

[84] K. Ackermann, Phase 2 VXD Volume - CAD Drawing, 2016. (Cited on page 80.)

[85] G. Bassi, Radiation monitor with diamond sensors for the Belle II experiment at SuperKEKB. PhD thesis, Trieste U., 2018. (Cited on page 81.)

[86] F. D. Capua, C. L. Licata, and L. Vitale, First Results for Total Integral Dose measurement with Radio-chromic Films in Phase 2, Oct., 2018. https://kds.kek.jp/indico/event/28981/contributions/102438/ attachments/81904/97205/RCF-B2GMOctober2018-BEAST .pdf. (Cited on page 82.)

[87] H. Schreeck et al., Effects of gamma irradiation on DEPFET pixel sensors for the Belle II experiment, Nucl. Instrum. Methods Phys. Res., Sect. A 959 (2020) 163522. (Cited on pages 109, 117, 124, 125, 126, 127, 129, 130, 131, 134, 140, 142 , and 143.)

[88] VARIOSCAN high resolution Models 3021, 3021-ST and 3022. (Cited on page 110.)

[89] J. Allison et al., Recent developments in Geant4, Nucl. Instrum. Methods Phys. Res. A 835 (2016) 186 - 225. (Cited on page 113.)

[90] F. Salvat, J. M. Fernández-Varea, and J. Sempau, PENELOPE-2008: A code system for Monte Carlo simulation of electron and photon transport, Workshop Proceedings 4 (2006) 7. (Cited on page 113.)

[91] M. Guthoff, O. Brovchenko, W. de Boer, A. Dierlamm, T. Müller, A. Ritter, M. Schmanau, and H.-J. Simonis, Geant4 simulation of a filtered $X$-ray source for radiation damage studies, Nucl. Instrum. Methods Phys. Res., Sect. A 675 (2012) 118 - 122. (Cited on page 116.)

[92] A. Qamesh, X-ray Irradiation and Calibration of the RD53A Pixel Read-out Chip, Master's thesis, Friedrich-Wilhelms-Universität Bonn, 2019. (Cited on page 116.) 


\section{BIBLIOGRAPHY}

[93] AXUVHS5 Photodiode $1 \mathrm{~mm}^{2}$, https://optodiode.com/pdf/AXUVHS5.pdf. (Cited on page 116.)

[94] J. Hubbell and S. Seltzer, Tables of x-ray mass attenuation coefficients and mass energy-absorption coefficients $1 \mathrm{keV}$ to $20 \mathrm{meV}$ for elements $z=1$ to 92 and 48 additional substances of dosimetric interest.. (Cited on page 117.)

[95] I. Peric, P. Fischer, and C. Kreidl, DCD-Bv4-Pipeline Reference Manual. Document revision 1, chip revision 4.0. University of Heidelberg, 2015. (Cited on pages 122 and 128.)

[96] A. Ritter et al., Investigations on radiation hardness of DEPFET sensors for the Belle II detector, Nucl. Instrum. Methods Phys. Res. A 730 (2013) $79-83$. (Cited on page 127.)

[97] T. P. Kleinohl, Characterization of DEPFET Pixel Devices under Electron Irradiation, Master's thesis, Physikalisches Institut der Universität Bonn, 2012. (Cited on page 127.)

[98] M. Lemarenko et al., Test results of the Data Handling Processor for the DEPFET Pixel Vertex Detector, J. Instrum. 8 (2013) C01032. (Cited on page 128.)

[99] I. Peric, P. Fischer, and C. Kreidl, SwitcherB18 (Gated Mode) Reference Manual. Document revision 3.4, chip design 2.0. University of Heidelberg, 2014. (Cited on page 128.)

[100] X. Mougeot, BetaShape: A new code for improved analytical calculations of beta spectra, EPJ Web of Conferences 146 (2017) 12015. (Cited on page 135.)

[101] X. Mougeot, Towards high-precision calculation of electron capture decays, Appl. Radiat. Isot. 154 (2019) 108884. (Cited on page 135.)

[102] R. Diener et al., The DESY II test beam facility, Nucl. Instrum. Methods Phys. Res. A 922 (2019) 265-286. (Cited on pages 140 and 141.)

[103] P. Wieduwilt et al., Test beam measurements of (final-type) DEPFET pixel detector modules for the Belle II experiment, in preparation (2020). (Cited on page 140.)

[104] H. Jansen et al., Performance of the EUDET-type beam telescopes, EPJ Tech. Instrum. 3 (2016) 7. (Cited on pages 140 and 142.)

[105] M. Garcia-Sciveres et al., The FE-I4 pixel readout integrated circuit, Nucl. Instrum. Methods Phys. Res., Sect. A 636 (2011) S155 - S159, 7th International Hiroshima Symposium on the Development and Application of Semiconductor Tracking Detectors. (Cited on page 141.) 
[106] B. Schwenker, test beam software framework (TBSW), 2019. https://bitbucket.org/testbeam/tbsw. (Cited on page 141.)

[107] R. E. Kalman, A New Approach to Linear Filtering and Prediction Problems, Journal of Basic Engineering 82 (1960) 35-45. (Cited on page 141.)

[108] B. Schwenker, Development and validation of a model for the response of the Belle II vertex detector. PhD thesis, Oct, 2014. (Cited on page 141.)

[109] U. Stolzenberg, Radiation length measurements with high-resolution telescopes. PhD thesis, 2019. (Cited on page 141.) 

Appendices 



\section{APPENDIX $A$}

\section{ADC Transfer Curves}

As described in section 4.5.3 ADC transfer curves are used to quantify the performance of the DCD. In the following the most important quality criteria are described and example curves are shown.

For each input value (measurement point) of the transfer curve multiple readings are taken $(\approx 100)$ which allows the calculation of a noise value. For the noise grading of the entire curve the median noise of all measurement points of the curve is used.

The linearity criterion is based on the integral non-linearity (INL) which can be calculated for each ADC code $i$ as

$$
\mathrm{INL}_{i}=\sum_{j=0}^{i} \mathrm{DNL}_{j} .
$$

This quantity is a sum over the differential non-linearity (DNL) values which are defined for each ADC code $i$ :

$$
\mathrm{DNL}_{i}=\frac{f_{i}}{f_{\text {mean }}}-1 .
$$

Here $f_{i}$ is the number of occurrences for a given ADC code $i$ and $f_{\text {mean }}$ the mean number of occurrences for all codes. The linearity for the entire ADC transfer curve is defined as

$$
\mathrm{INL}_{\mathrm{pp}}=\left|\max \left(\mathrm{INL}_{i}\right)-\min \left(\mathrm{INL}_{i}\right)\right| .
$$

The DNL is also used to identify code gaps in the ADC curves, as missing output codes have a DNL of 0 . Another criterion is the covered code range. This can be checked easily by looking at the lowest and highest output codes returned by the DCD. 


\section{A. ADC Transfer Curves}

Additionally, a check for digital communication errors (bit errors) is performed. This kind of error can be identified by searching for output values far away from the nominal ADC curve. Assuming the delay elements of the DHP are configured correctly this error is rather uncommon.

Table A.1 shows the values for the different quality criteria that are applied to the transfer curves. Based on this criteria a curve is either grade-A (passes all tests), grade-B (fails at least one test) or grade-F (no response at all).

Figure A.1 shows an example of an optimal ADC curve while figs. A.2 to A.4 show examples of the most common errors observed during the DCD parameter optimisation.

\begin{tabular}{rrrrrr}
\hline & Code Range & Noise & \# Code Gaps & INL $_{\text {pp }}$ & \# Digital Errors \\
\hline Value & $10-240 \mathrm{ADU}$ & $<2 \mathrm{ADU}$ & 0 & $<20$ & 0 \\
\hline
\end{tabular}

Table A.1.: Criteria used for the classification of ADC transfer curves. If all criteria are met, a curve is classified grade-A. If one or more of the quality criteria are not met, the curve is classified as grade-B. Curves that show no response at all are marked as grade-F.

\section{A.1. Transfer Curve Examples}

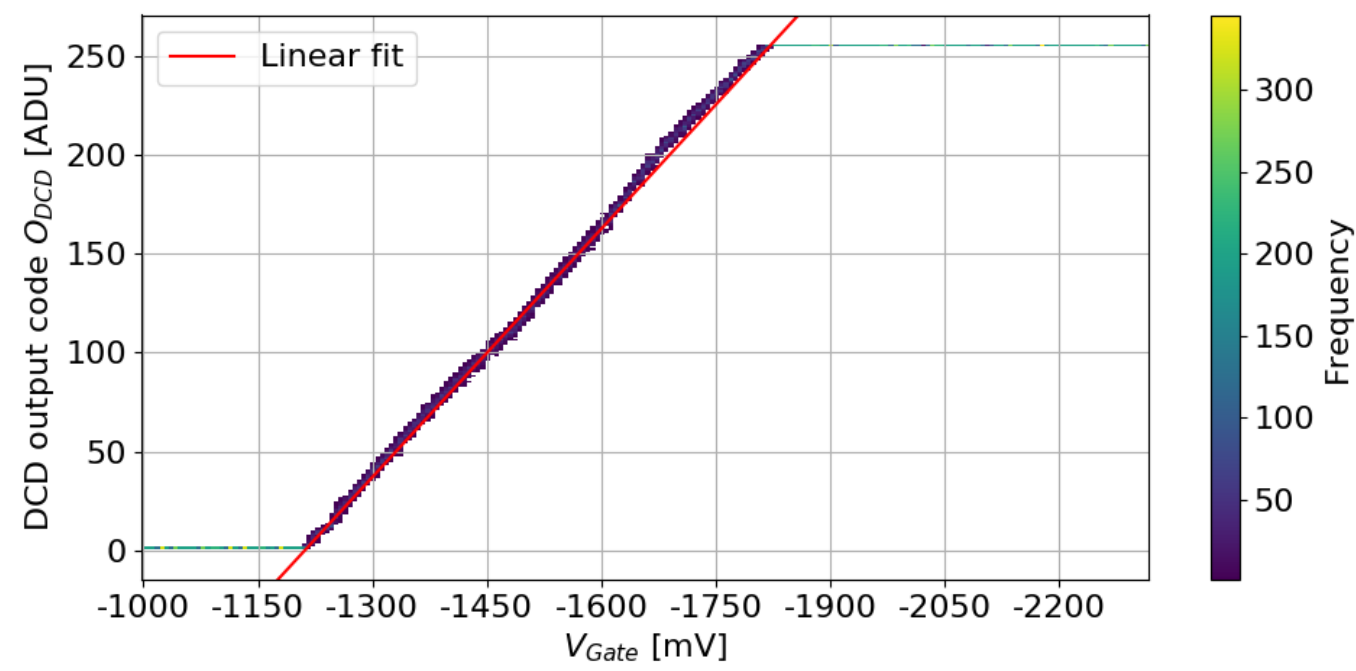

Figure A.1.: Example of an ADC transfer curve that passes all quality criteria. There are no gaps in the curve, i.e. no missing output codes, it has a good linearity and covers the complete output code range. 


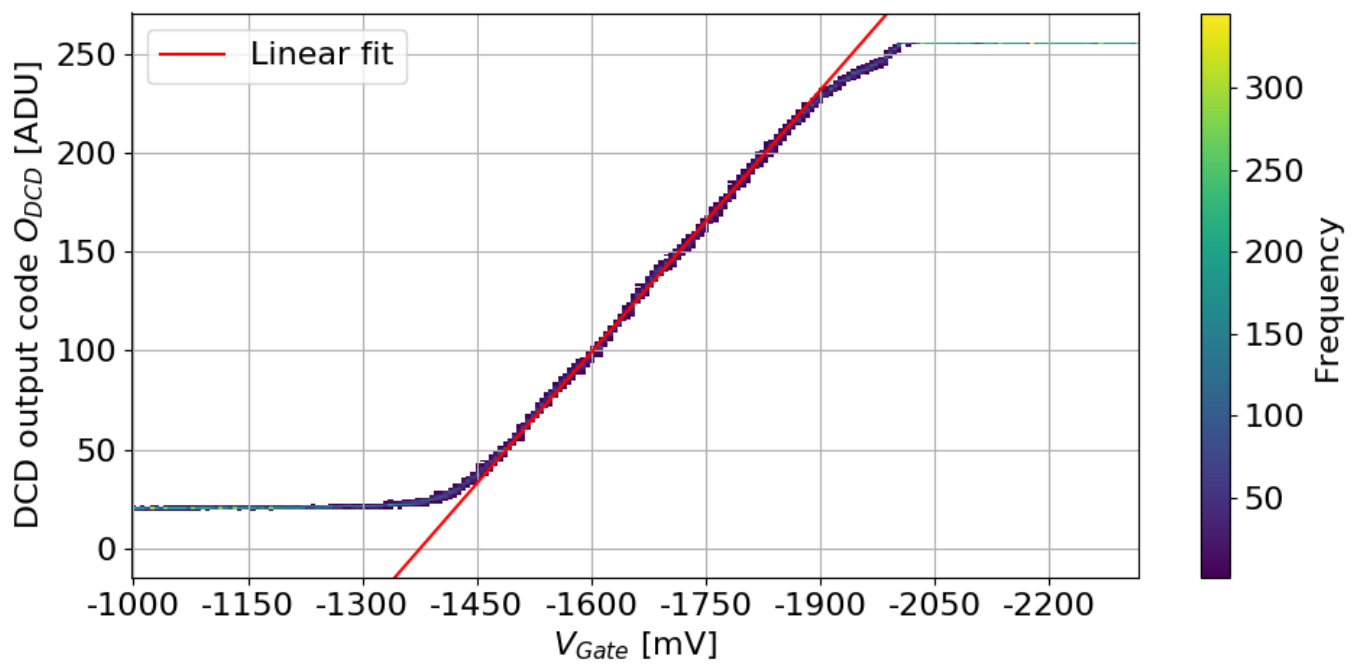

Figure A.2.: Example of an ADC transfer curve that has a reduced range in its output code range (no output codes below $\approx 20 \mathrm{ADU}$.

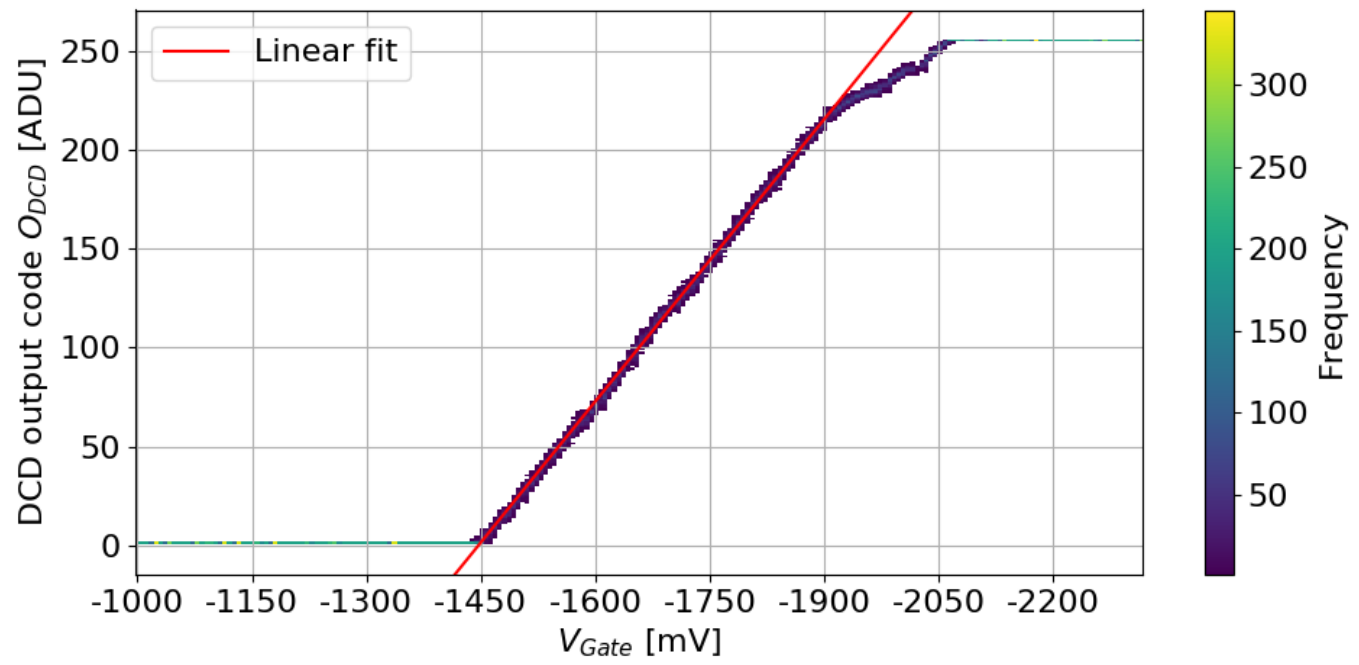

Figure A.3.: Example of an ADC transfer curve that has non-optimal linearity for high output values. 
A. ADC Transfer Curves

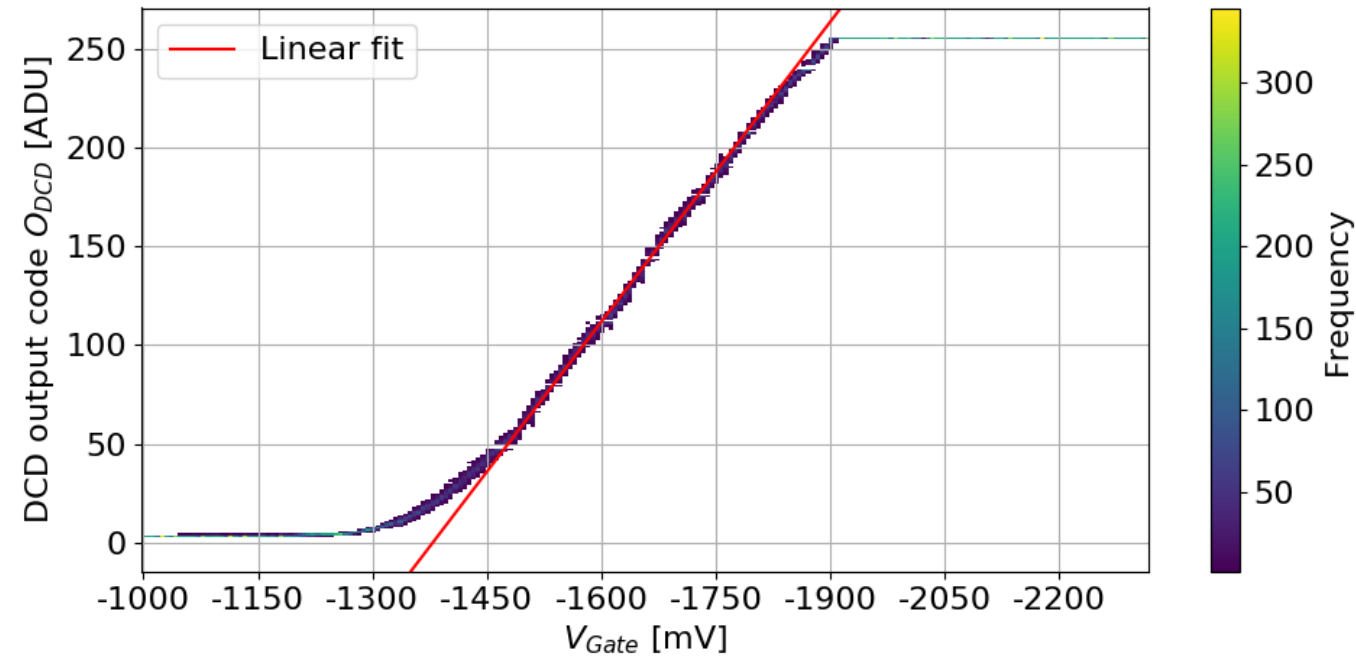

Figure A.4.: Example of an ADC transfer curve that has multiple missing output codes (white gaps in the curve). 
APPENDIX B

Total lonizing Dose Estimation

\section{B.1. Residuals}
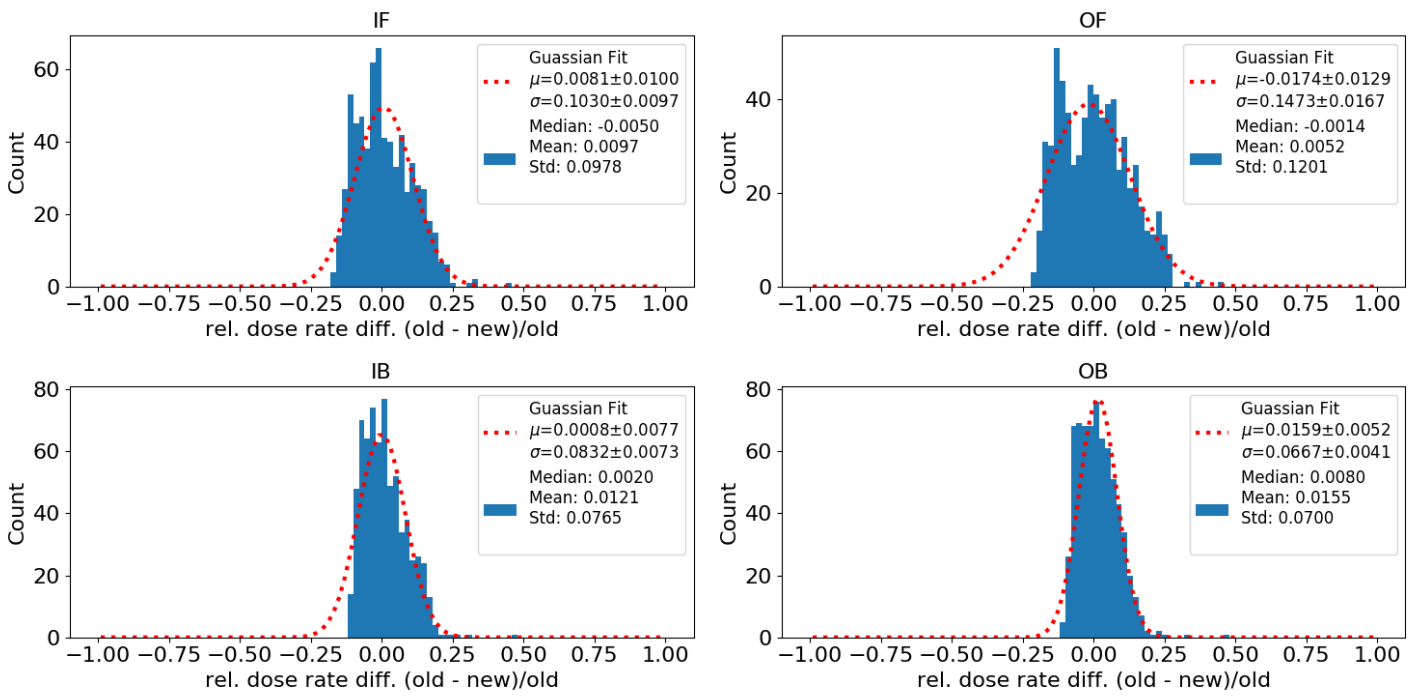

Figure B.1.: Comparison of original PXD dose measurement (old) and calculated PXD dose based on beam currents and diamond data (new) from 2018-04-19 to 2018-05-15. 

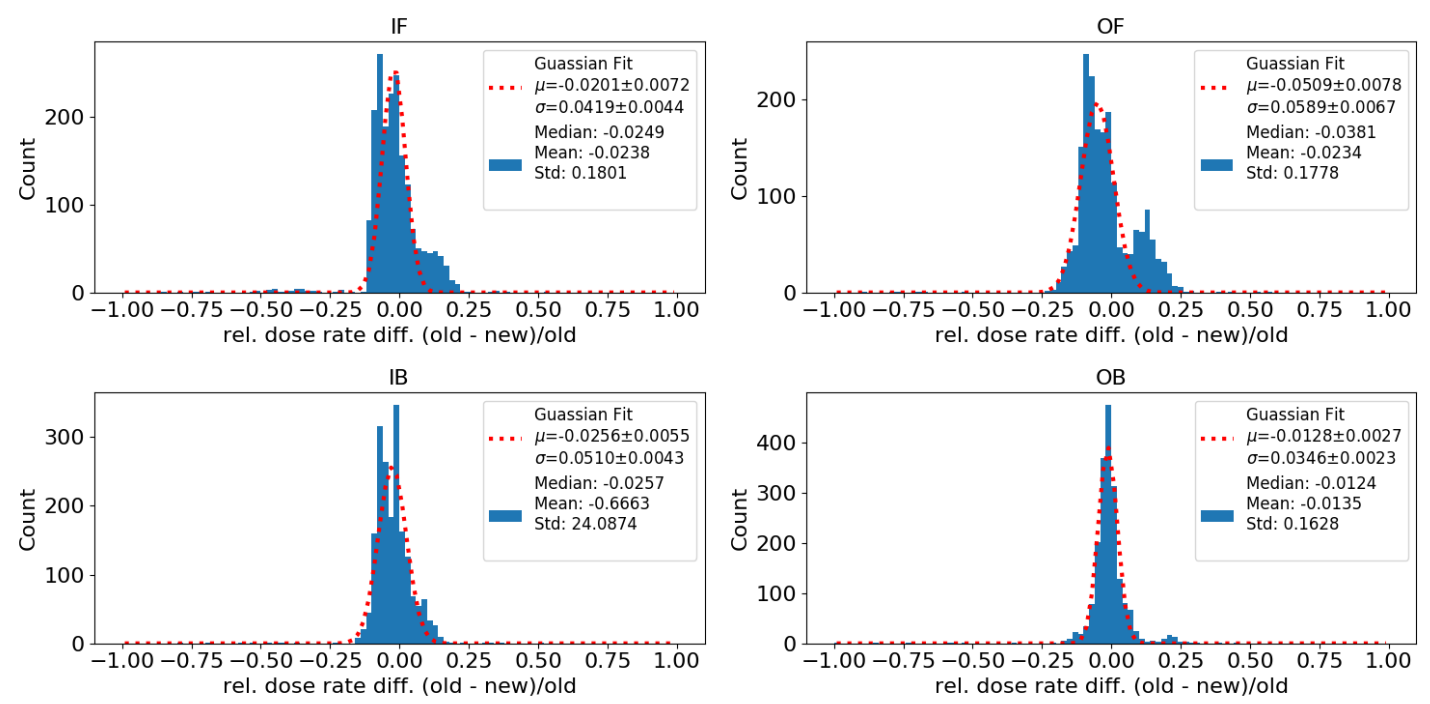

Figure B.2.: Comparison of original PXD dose measurement (old) and calculated PXD dose based on beam currents and diamond data (new) from 2018-05-16 to 2018-05-25.
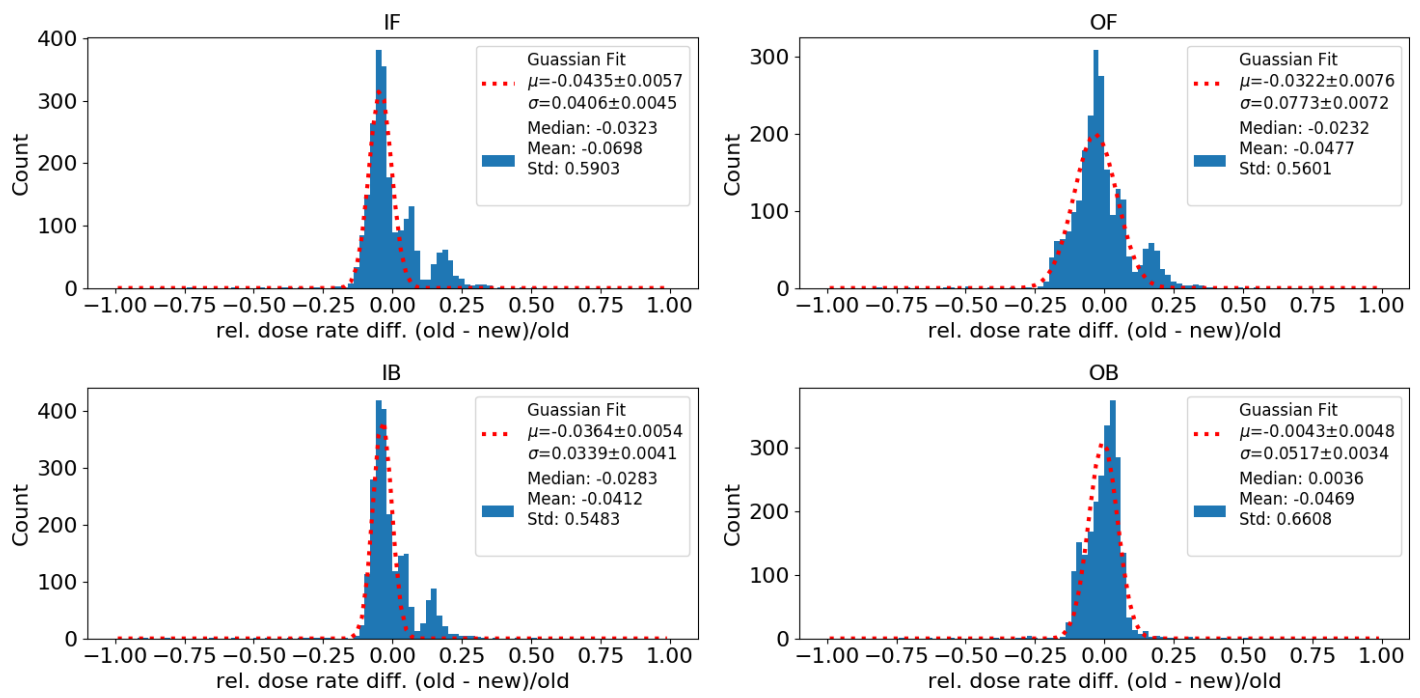

Figure B.3.: Comparison of original PXD dose measurement (old) and calculated PXD dose based on beam currents and diamond data (new) from 2018-05-26 to 2018-06-01. 

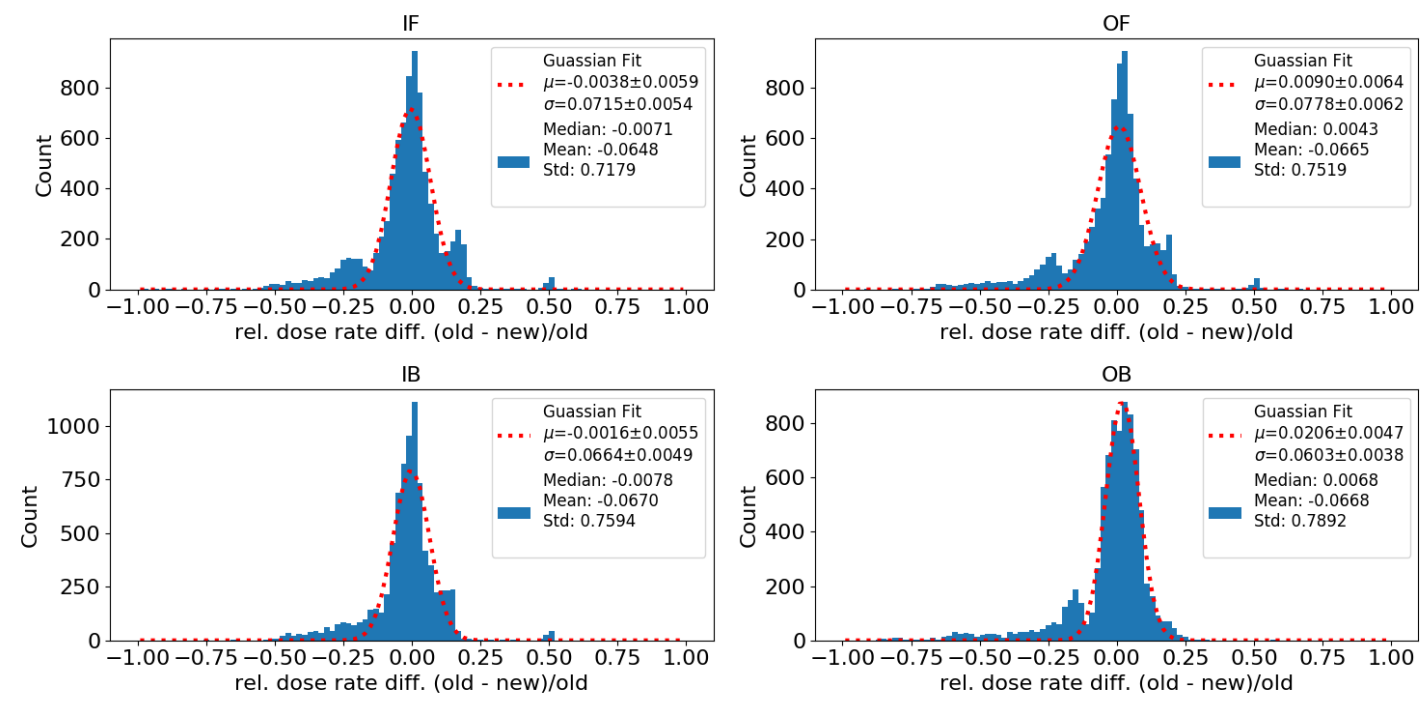

Figure B.4.: Comparison of original PXD dose measurement (old) and calculated PXD dose based on beam currents and diamond data (new) from 2018-06-02 to 2018-07-01.
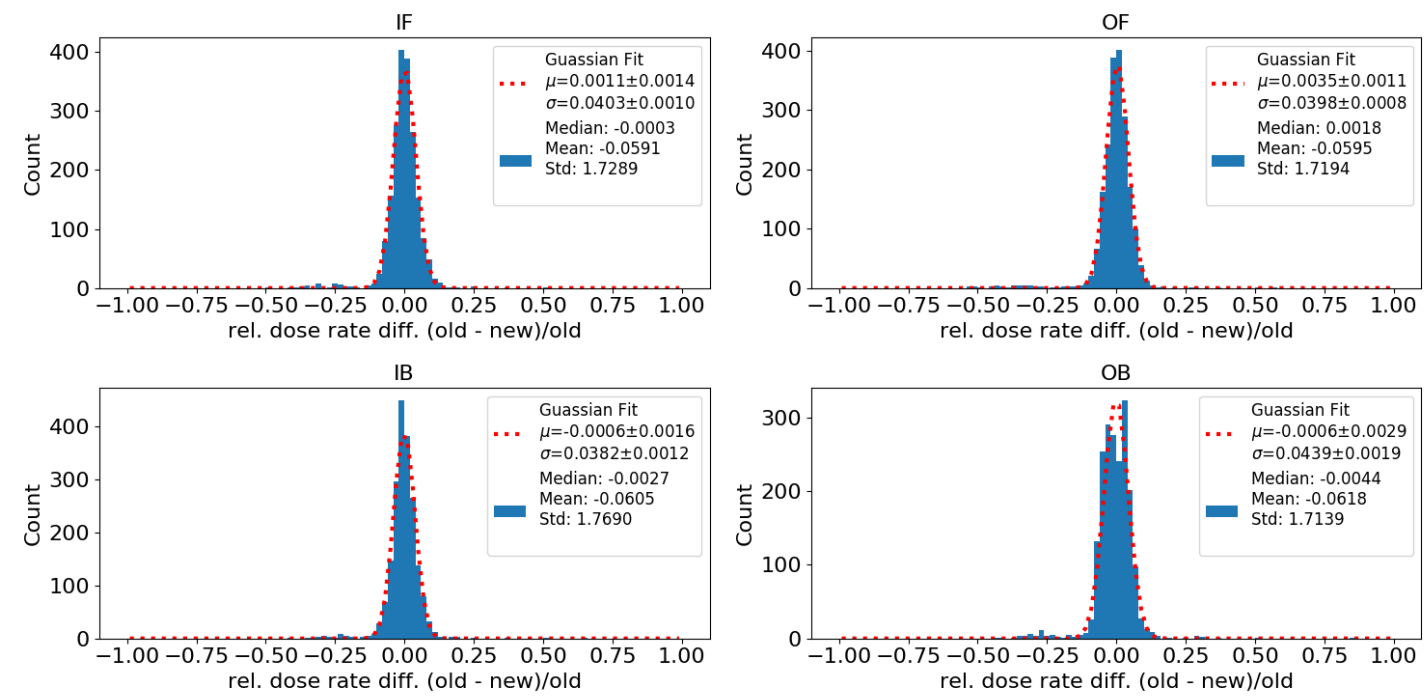

Figure B.5.: Comparison of original PXD dose measurement (old) and calculated PXD dose based on beam currents and diamond data (new) from 2018-07-02 to 2018-07-10. 



\section{C.1. System Gain Evolution}

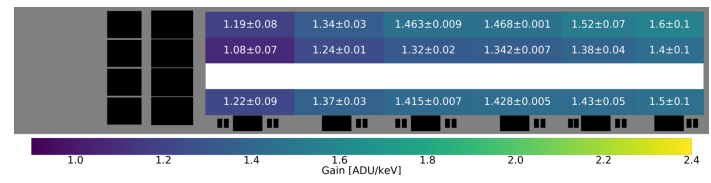

(a) Step 1 ( $\approx 50$ Gy)

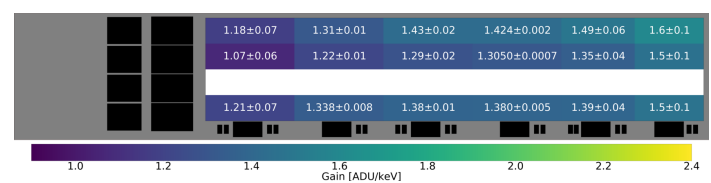

(c) Step 3 ( $\approx 250$ Gy)

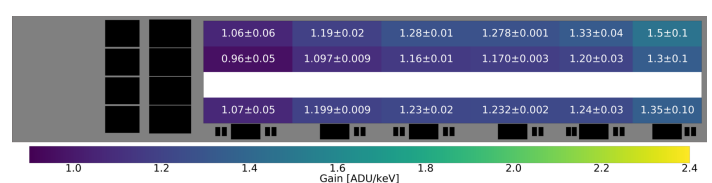

(e) Step $5(\approx 1 \mathrm{kGy})$

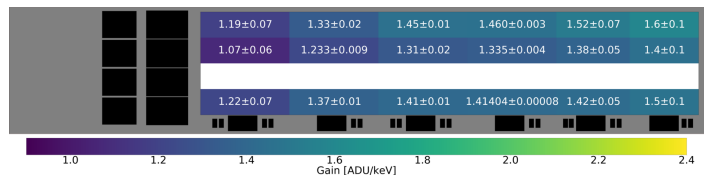

(b) Step 2 ( $\approx 100$ Gy)

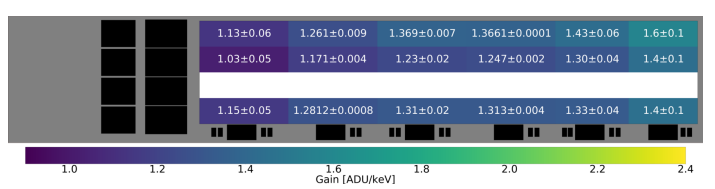

(d) Step 4 ( $\approx 500$ Gy)

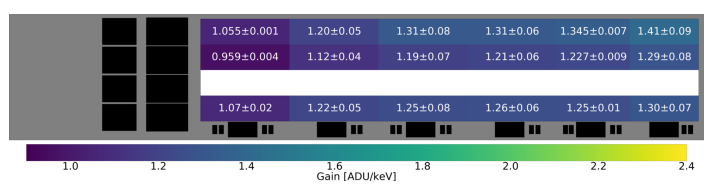

(f) Step $6(\approx 2 \mathrm{kGy})$

Figure C.1.: Gain evolution. 


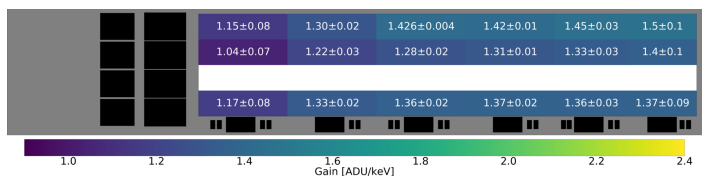

(a) Step 7 ( $\approx 5 \mathrm{kGy})$

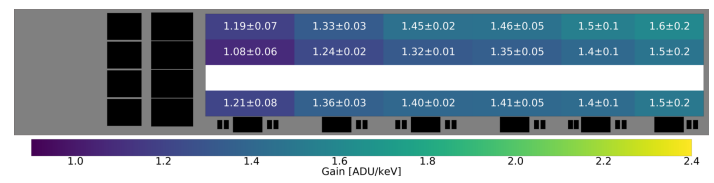

(b) Step $8(\approx 8 \mathrm{kGy})$

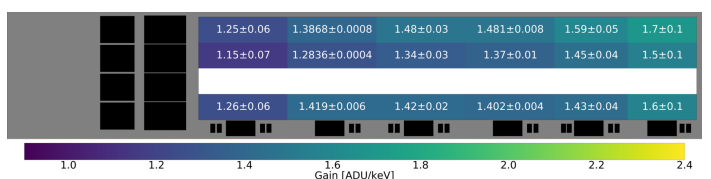

(c) Step 9 ( $\approx 11 \mathrm{kGy})$

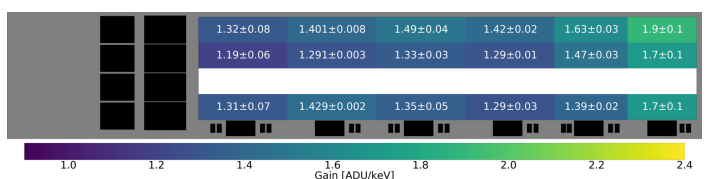

(e) Step $11(\approx 17 \mathrm{kGy})$

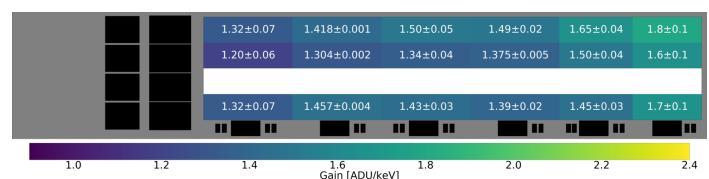

(d) Step $10(\approx 14 \mathrm{kGy})$

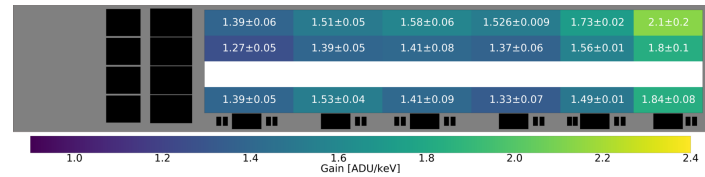

(f) Step $12(\approx 20 \mathrm{kGy})$

Figure C.2.: Gain evolution.

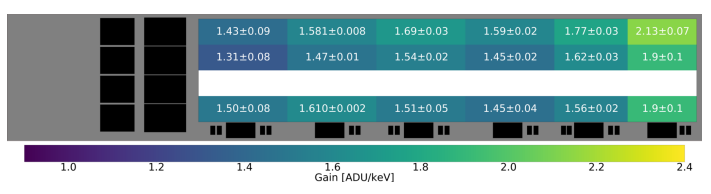

(a) Step $13(\approx 30 \mathrm{kGy})$

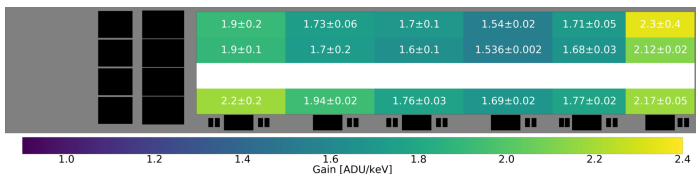

(c) Step $15(\approx 100 \mathrm{kGy})$

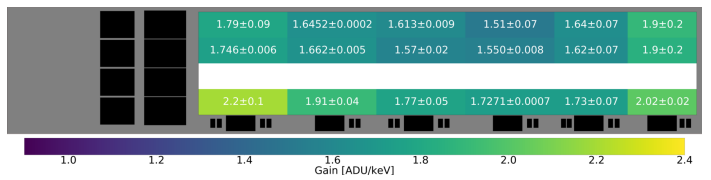

(e) Step $17(\approx 184 \mathrm{kGy})$

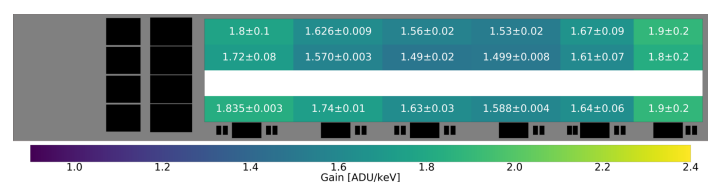

(b) Step 14 ( $\approx 58 \mathrm{kGy})$

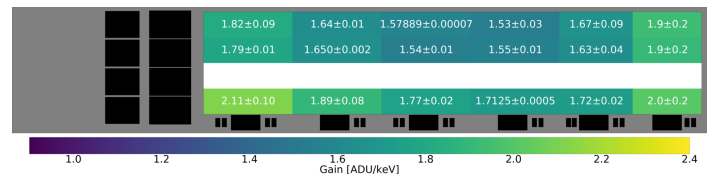

(d) Step $16(\approx 144 \mathrm{kGy})$

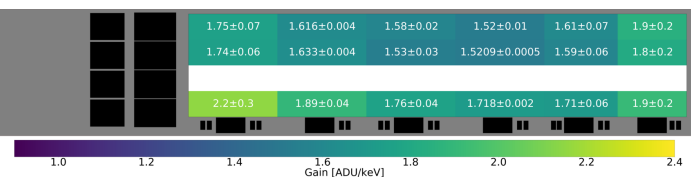

(f) Step $18(\approx 216 \mathrm{kGy})$

Figure C.3.: Gain evolution. 


\section{C.2. Signal-to-Noise Evolution}

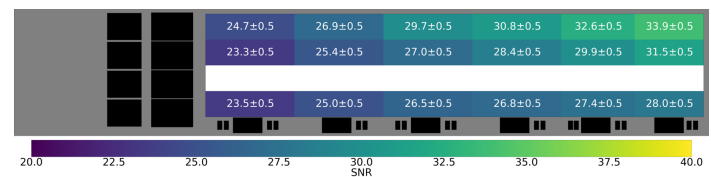

(a) Step 1 ( $\approx 50$ Gy)

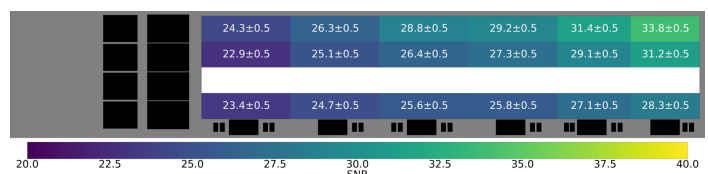

(c) Step 3 ( $\approx 250$ Gy)

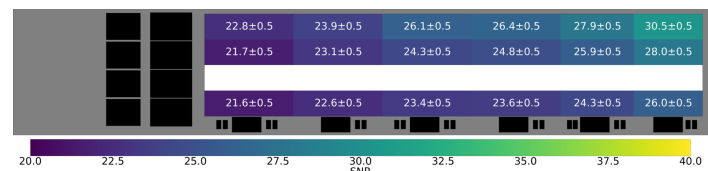

(e) Step 5 ( $\approx 1 \mathrm{kGy})$

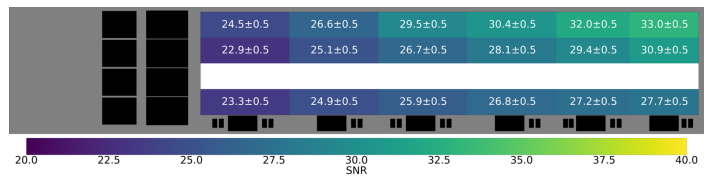

(b) Step 2 ( $\approx 100$ Gy)

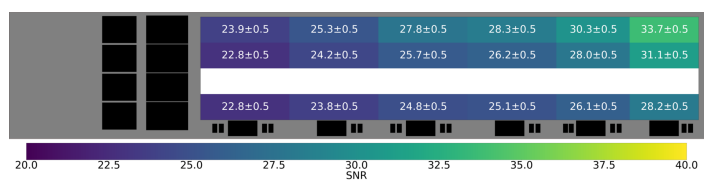

(d) Step 4 ( $\approx 500$ Gy)

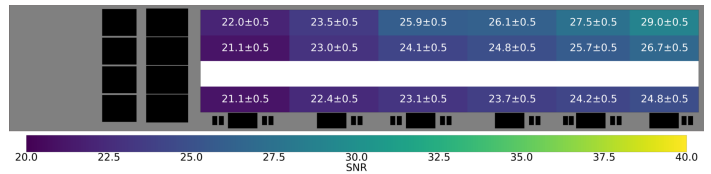

(f) Step $6(\approx 2 \mathrm{kGy})$

Figure C.4.: SNR evolution with the $\mathrm{Cd}^{109}$ source. 


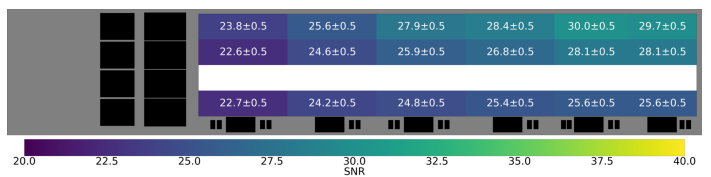

(a) Step 7 ( $\approx 5 \mathrm{kGy})$

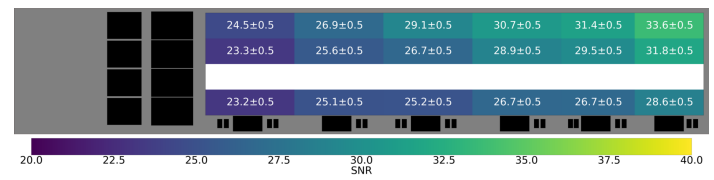

(b) Step $8(\approx 8 \mathrm{kGy})$

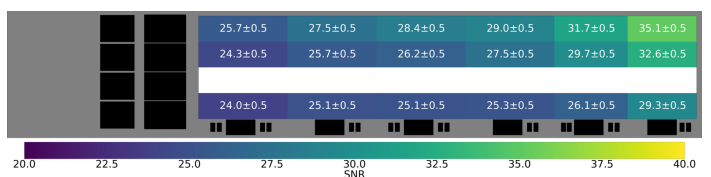

(c) Step 9 ( $\approx 11 \mathrm{kGy})$

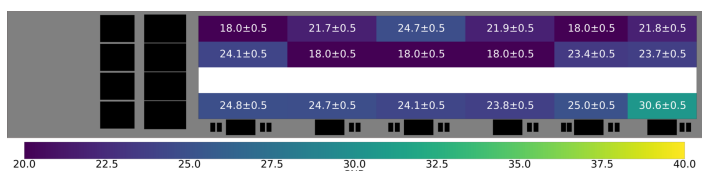

(e) Step $11(\approx 17 \mathrm{kGy})$

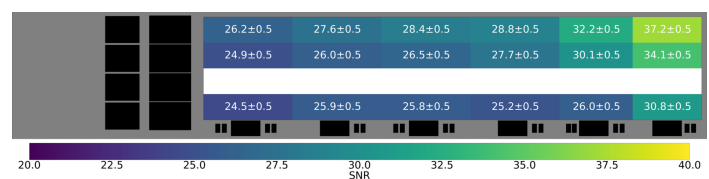

(d) Step 10 ( $\approx 14$ kGy)

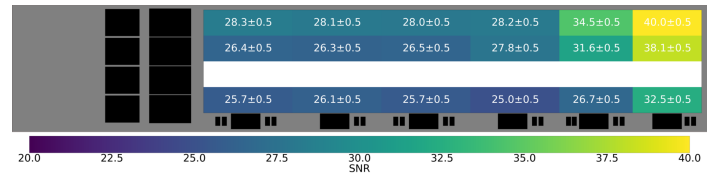

(f) Step $12(\approx 20 \mathrm{kGy})$

Figure C.5.: SNR evolution with the $\mathrm{Cd}^{109}$ source.

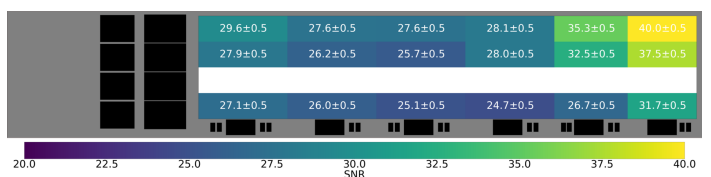

(a) Step $13(\approx 30$ kGy $)$

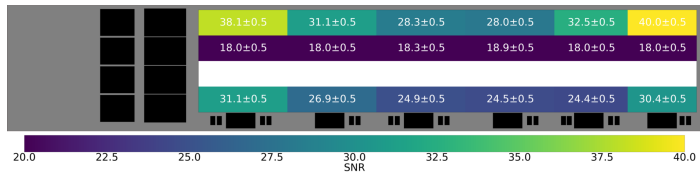

(c) Step $15(\approx 100 \mathrm{kGy})$

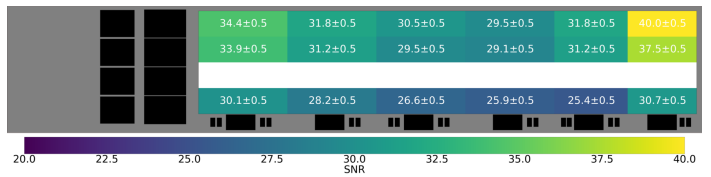

(e) Step $17(\approx 184 \mathrm{kGy})$

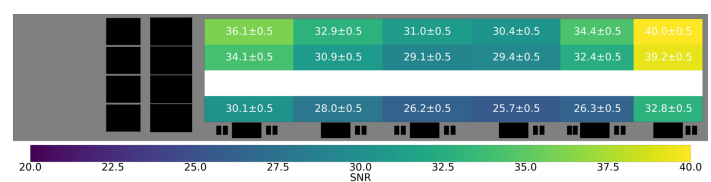

(b) Step 14 ( $\approx 58 \mathrm{kGy})$

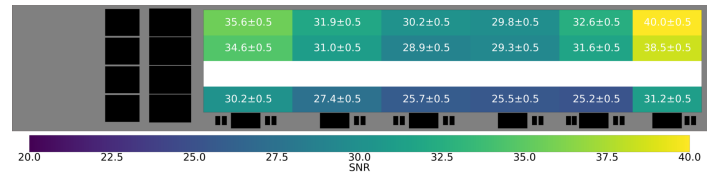

(d) Step $16(\approx 144 \mathrm{kGy})$

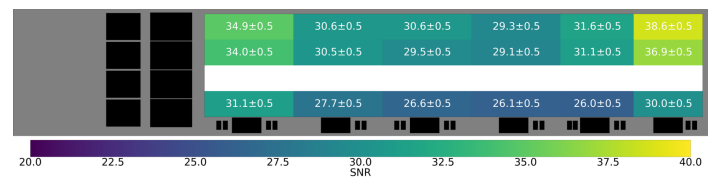

(f) Step $18(\approx 216 \mathrm{kGy})$

Figure C.6.: SNR evolution with the $\mathrm{Cd}^{109}$ source. 


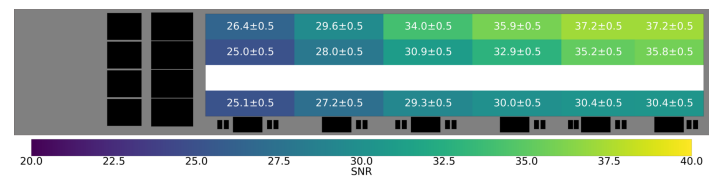

(a) Step 1 ( $\approx 50$ Gy)

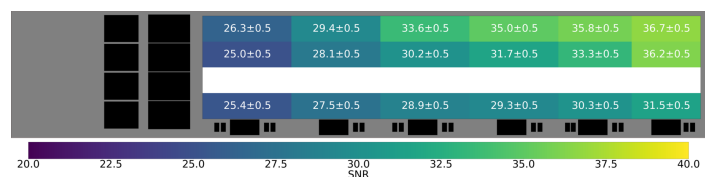

(c) Step 3 ( $\approx 250$ Gy)

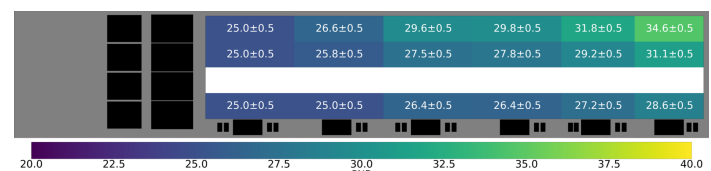

(e) Step $5(\approx 1 \mathrm{kGy})$

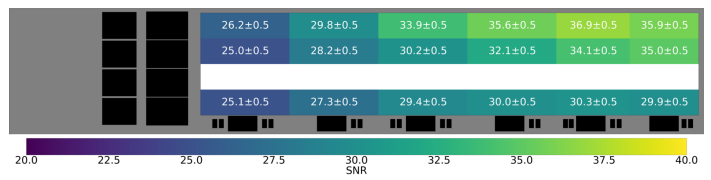

(b) Step 2 ( $\approx 100$ Gy)

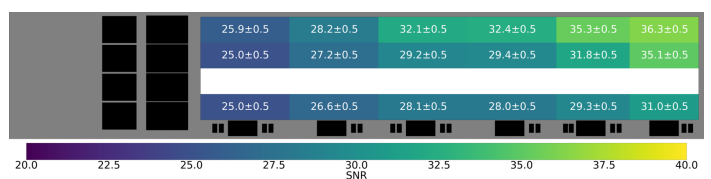

(d) Step 4 ( $\approx 500$ Gy)

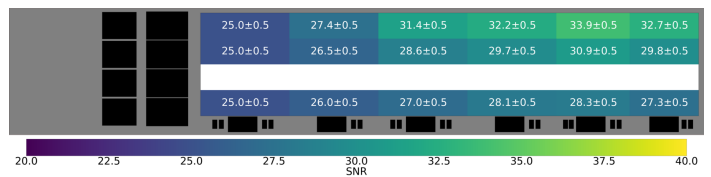

(f) Step $6(\approx 2 \mathrm{kGy})$

Figure C.7.: SNR evolution with the $\mathrm{Sr}^{90}$ source.

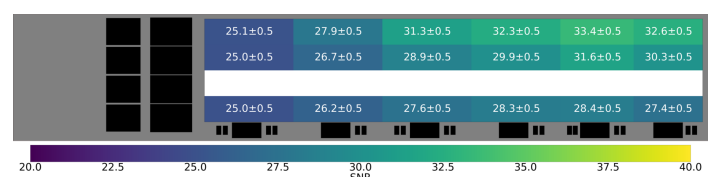

(a) Step 7 ( $\approx 5 \mathrm{kGy})$

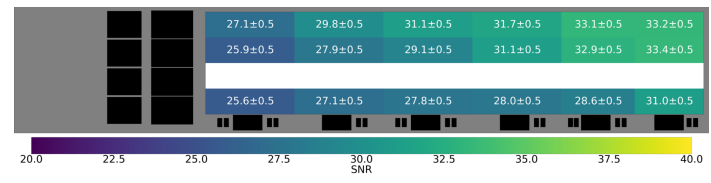

(c) Step $9(\approx 11 \mathrm{kGy})$

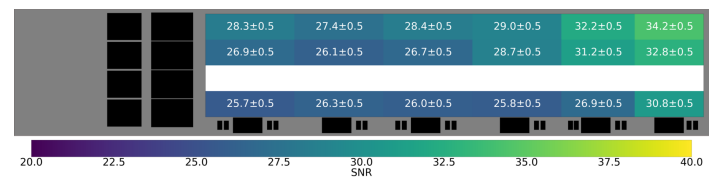

(e) Step 11 ( $\approx 17 \mathrm{kGy})$

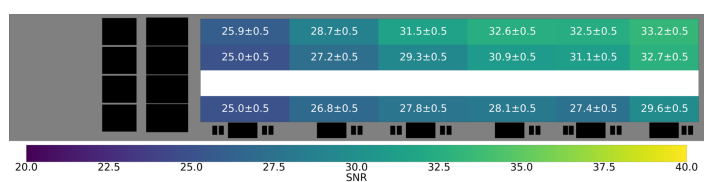

(b) Step $8(\approx 8 \mathrm{kGy})$

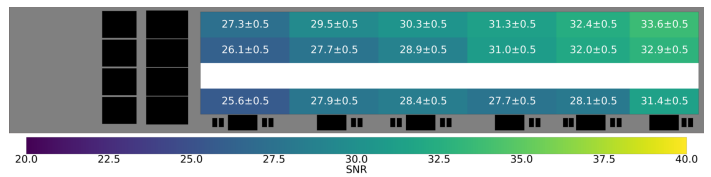

(d) Step $10(\approx 14 \mathrm{kGy})$

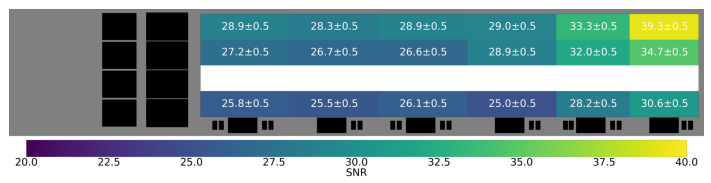

(f) Step $12(\approx 20 \mathrm{kGy})$

Figure C.8.: SNR evolution with the $\mathrm{Sr}^{90}$ source. 
C. Irradiation Campaign

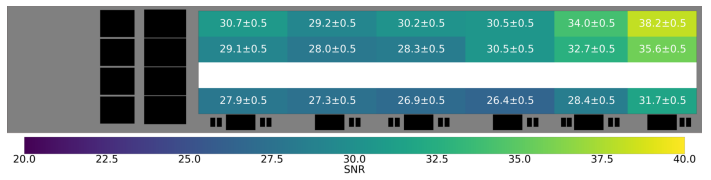

(a) Step $13(\approx 30 \mathrm{kGy})$

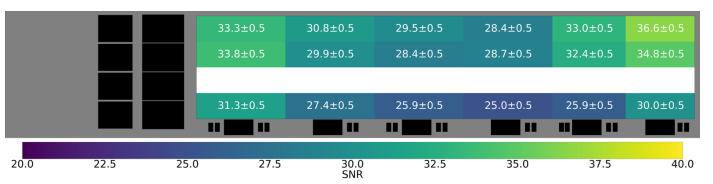

(c) Step $15(\approx 100 \mathrm{kGy})$

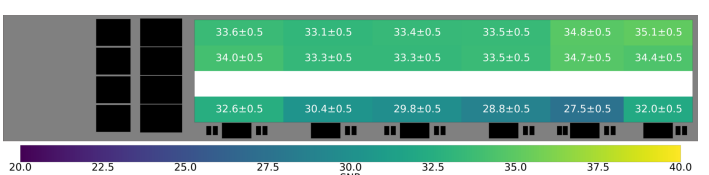

(e) Step $17(\approx 184 \mathrm{kGy})$

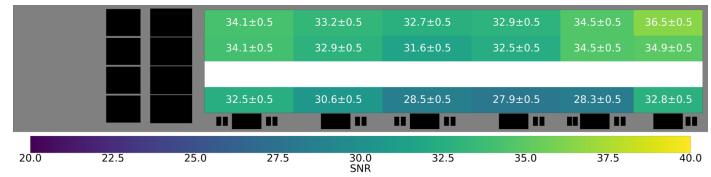

(b) Step $14(\approx 58 \mathrm{kGy})$

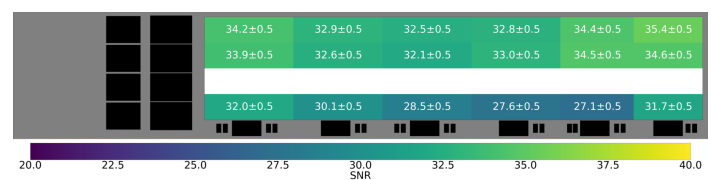

(d) Step $16(\approx 144 \mathrm{kGy})$

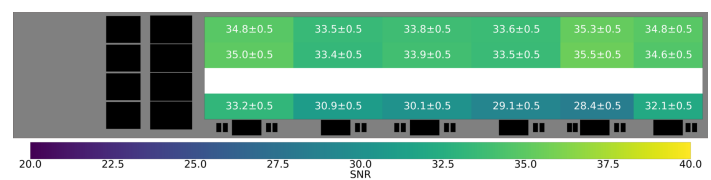

(f) Step $18(\approx 216 \mathrm{kGy})$

Figure C.9.: SNR evolution with the $\mathrm{Sr}^{90}$ source. 


\section{Acknowledgements}

First off, I would like to thank Prof. Dr. Ariane Frey for being my supervisor over the last four and a half years and giving me the chance to work on this interesting topic in her working group. I would also like to thank PD Dr. Jörn Große-Knetter for agreeing to be the co-referee of this thesis. Likewise my thanks go to other member of the examination committee Prof. Dr. Stan Lai, Prof. Dr. Wolfram Kollatschny, Prof. Dr. Hans-Christian Hofsäss and Dr. Carsten Niebuhr.

Thanks also to Benjamin Schwenker for his support and many stimulating discussions over the last years. His feedback, knowledge and ideas were always a big help and made many of the analyses in this thesis possible. Furthermore I want to thank Philipp Wieduwilt with whom I worked together on the DEPFET project here in Göttingen for his constructive criticism and practical suggestions.

I would also like to thank all the other members of the working group, Ulf, Uwe, Cesar, Julian, Noreen and Jan-Lucas. Working with you was a pleasure and I always enjoyed your company, being it in the office or during business trips and test beams. Our regular group activities after work like bowling or kart racing made the time here special.

I also want to thank all the other members of the DEPFET collaboration, especially Botho Paschen, Florian Lütticke, Patrick Ahlburg, Carlos Marinas, Ladislav Andricek and Rainer Richter for helping with many of the measurements and analyses presented in this thesis.

Lastly, I would like to thank my mother Susanne for her love and support over the last 30 years. Her encouragement and profound belief in my abilities allowed me to be where I am today. Of course I also want to thank Julia for her patience and emotional support especially over the last months. At the very end I want to express my gratitude to my grandparents Lydia and Dieter who were always there for me, I miss you. 
